\title{
IntechOpen
}

\section{Research Advances in Chaos Theory}

Edited by Paul Bracken 



\section{Research Advances in Chaos Theory}

Edited by Paul Bracken 

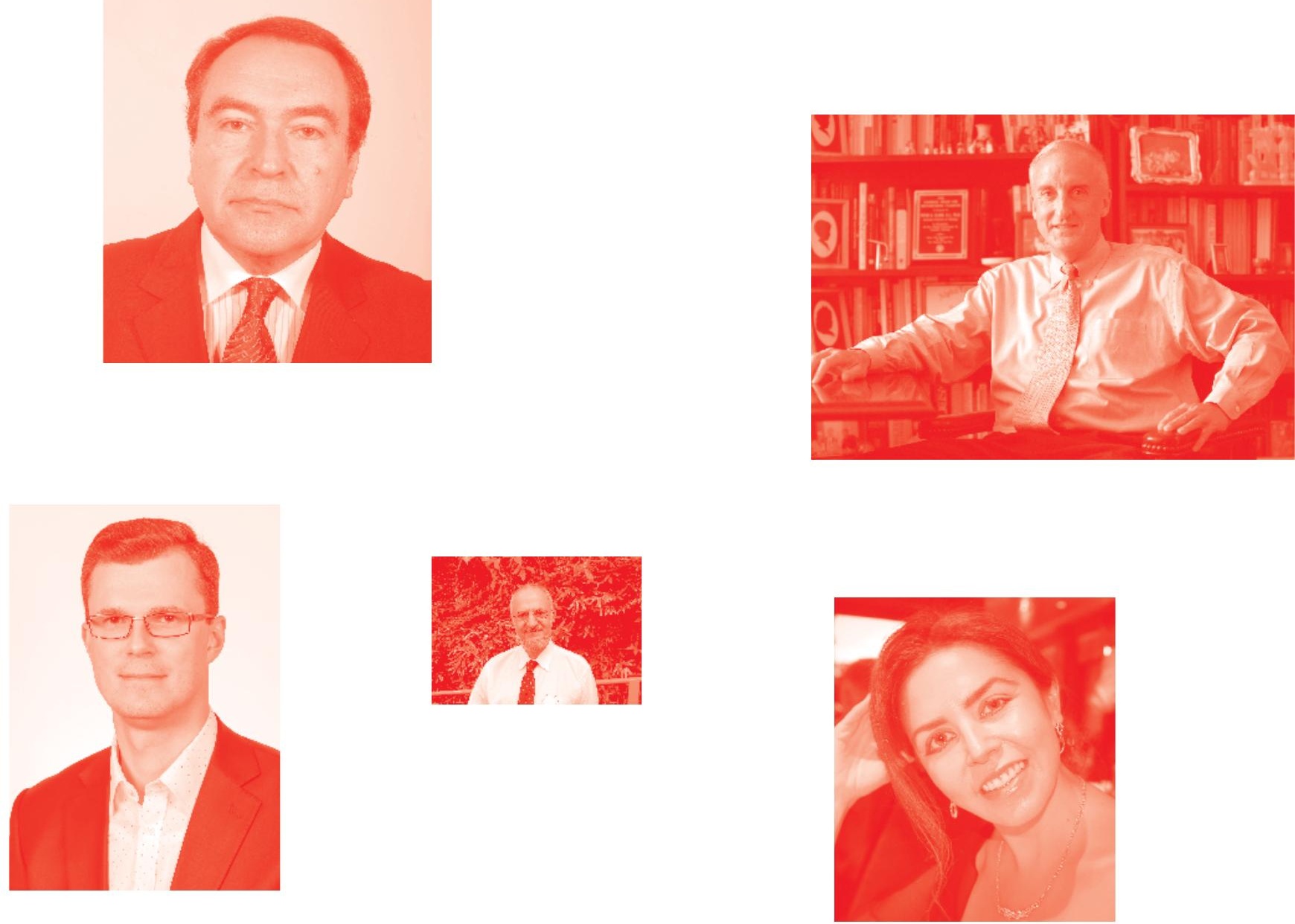

Supporting open minds since 2005
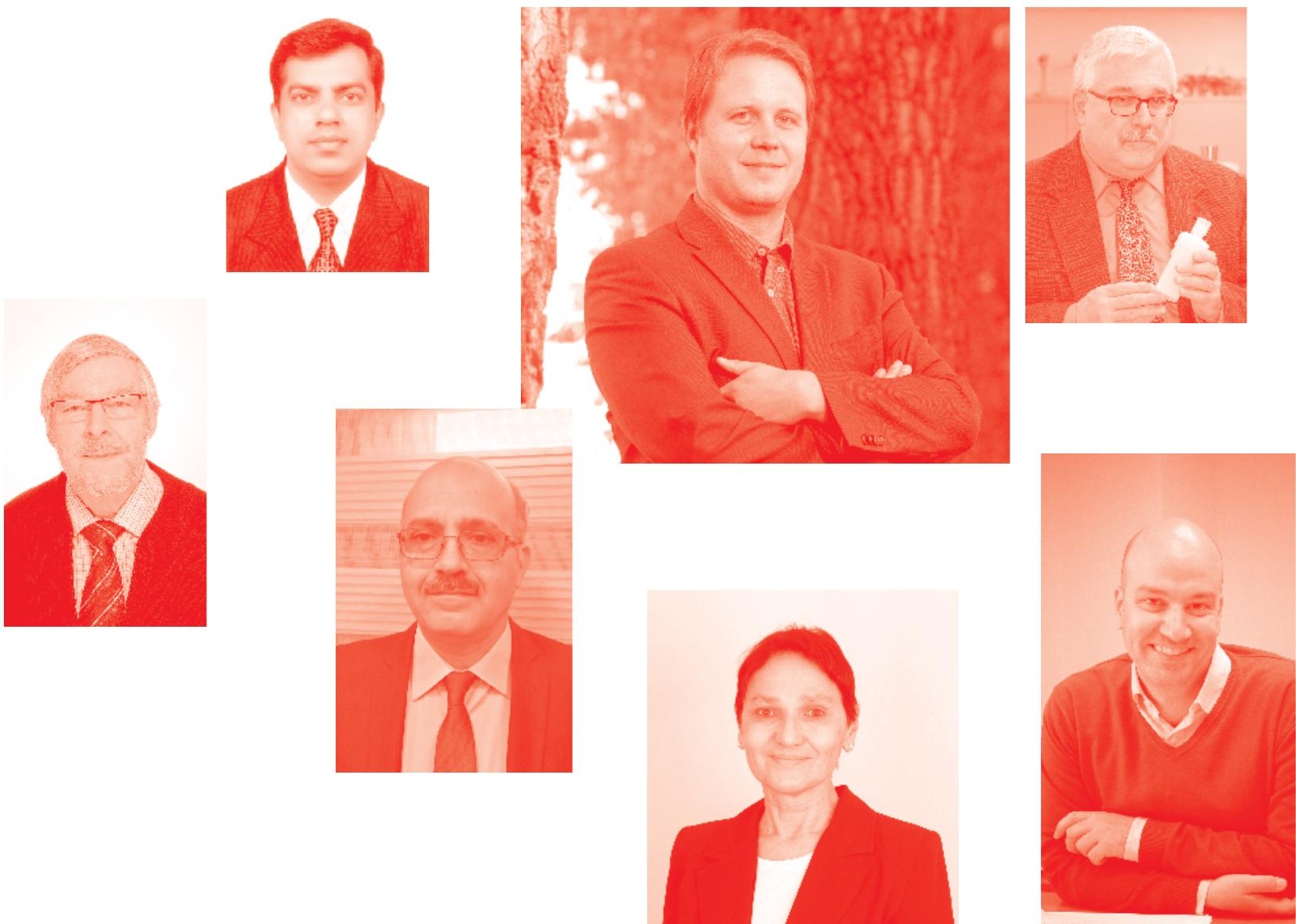
Research Advances in Chaos Theory

http : //dx . doi . org/10.5772/intechopen. 77590

Edited by Paul Bracken

\section{Contributors}

Justin Mibaile, Hubert Malwe, Vyacheslav O Vakhnenko, John Parkes, Dmitri Vengrovich, Joel Bruno Gonpe Tafo, Tabi Conrad Bertrand, Laurent Nana, Kofane Timoléon Crépin, Beatrice Venturi, Wei Zhou, Tong Chu, Paul Bracken, Valeri Kontorovich

() The Editor(s) and the Author(s) 2020

The rights of the editor(s) and the author(s) have been asserted in accordance with the Copyright, Designs and Patents Act 1988. All rights to the book as a whole are reserved by INTECHOPEN LIMITED . The book as a whole (compilation) cannot be reproduced, distributed or used for commercial or non-commercial purposes without INTECHOPEN LIMITED's written permission. Enquiries concerning the use of the book should be directed to INTECHOPEN LIMITED rights and permissions department (permissions@intechopen.com).

Violations are liable to prosecution under the governing Copyright Law .

\section{(cc) BY}

Individual chapters of this publication are distributed under the terms of the Creative Commons Attribution 3.0 Unported License which permits commercial use, distribution and reproduction of the individual chapters, provided the original author(s) and source publication are appropriately acknowledged. If so indicated, certain images may not be included under the Creative Commons license. In such cases users will need to obtain permission from the license holder to reproduce the material. More details and guidelines concerning content reuse and adaptation can be found at http : //www . intechopen . com/copyright-policy . html.

\section{Notice}

Statements and opinions expressed in the chapters are these of the individual contributors and not necessarily those of the editors or publisher. No responsibility is accepted for the accuracy of information contained in the published chapters. The publisher assumes no responsibility for any damage or injury to persons or property arising out of the use of any materials, instructions, methods or ideas contained in the book.

First published in London, United Kingdom, 2020 by IntechOpen IntechOpen is the global imprint of INTECHOPEN LIMITED, registered in England and Wales, registration number: 11086078 , 7th floor, 10 Lower Thames Street, London, EC3R 6AF, United Kingdom

Printed in Croatia

British Library Cataloguing-in-Publication Data

A catalogue record for this book is available from the British Library

Additional hard and PDF copies can be obtained from orders@intechopen .com

Research Advances in Chaos Theory

Edited by Paul Bracken

p. cm.

Print ISBN 978-1-78985-543-2

Online ISBN 978-1-78985-544-9

eBook (PDF) ISBN 978-1-83880-408-4 


\section{We are IntechOpen, \\ the world's leading publisher of Open Access books}

Built by scientists, for scientists

\section{$4,700+$}

Open access books available

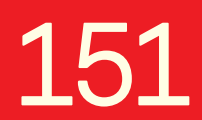

Countries delivered to
$120,000+$

International authors and editors

Our authors are among the

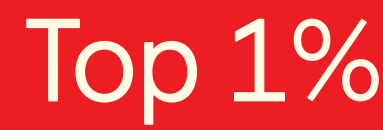

most cited scientists

Contributors from top 500 universities
$135 \mathrm{M}+$

Downloads

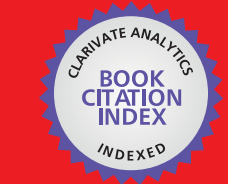

WEB OF SCIENCE ${ }^{\mathrm{MM}}$

Selection of our books indexed in the Book Citation Index in Web of Science ${ }^{\mathrm{TM}}$ Core Collection (BKCI)

\section{Interested in publishing with us? \\ Contact book.department@intechopen.com}

Numbers displayed above are based on latest data collected.

For more information visit www.intechopen.com 



\section{Meet the editor}

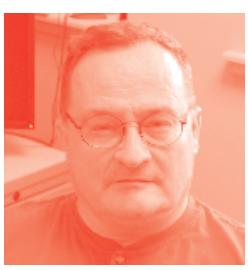

Dr. Paul Bracken is currently a professor in the Mathematics Department at the University of Texas, Edinburg, TX. He obtained his BSc from the University of Toronto and $\mathrm{PhD}$ from the University of Waterloo in Canada. His research interests include mathematical problems in quantum mechanics and quantum field theory, differential geometry and partial differential equations as well as their overlap with the area of gravity. He has published more than 150 papers in journals and books and has given about 20 talks at various meetings and conferences at different levels. This is the fifth volume he has contributed with IntechOpen. 



\section{Contents}

$\begin{array}{lll}\text { Preface } & \text { XIII }\end{array}$

Section 1

Chaos in Quantum Mechanics

Chapter 1

Introductory Chapter: Dynamical Symmetries and Quantum Chaos

by Paul Bracken

Section 2

$\begin{array}{ll}\text { Nonlinear Dynamics } & 11\end{array}$

$\begin{array}{ll}\text { Chapter } 2 & 13\end{array}$

Loop-like Solitons

by Vyacheslav O. Vakhnenko, E. John Parkes and

Dmitri B. Vengrovich

Chapter 3

Complex Dynamical Behavior of a Bounded Rational Duopoly

Game with Consumer Surplus

by Wei Zhou and Tong Chu

Chapter 4

Nonlinear Dynamical Regimes and Control of Turbulence through the Complex Ginzburg-Landau Equation

by Joël Bruno Gonpe Tafo, Laurent Nana, Conrad Bertrand Tabi and Timoléon Crépin Kofané

Chapter 5

Ubiquitous Filtering for Nonlinear Problems

by Valeri Kontorovich and Fernando Ramos-Alarcon

\section{Section 3}

Biology and Economics

Chapter 6

Chaos in Human Brain Phase Transition

by Mibaile Justin, Malwe Boudoue Hubert, Gambo Betchewe,

Serge Yamigno Doka and Kofane Timoleon Crepin 
Chapter 7

Global Indeterminacy and Invariant Manifolds Near Homoclinic Orbit to a Real Saddle

by Beatrice Venturi 


\section{Preface}

It is not exaggerating to state that the subject of chaos has reached nearly every branch of the natural sciences. It has become common to talk about chaotic weather patterns, chaotic evolution in population dynamics, and chaos in atomic physics and chemical reactions. The latter is perhaps a bit surprising at first, since at the atomic level, physics seems governed by the linear laws of quantum mechanics, while an essential ingredient of chaos is nonlinearity in the dynamical equations.

The current volume presents seven very good contributions to this area of research. One of the main contributions of the book is to illustrate the diversity of subjects that have been influenced by this area of research. Of these seven chapters, there are four that examine the issue of chaos in nonlinear and dynamical systems. These look at chaos as it manifests itself in various types of differential equations and systems of differential equations as well as its impact on mathematics in general. There is also a chapter that briefly discusses chaos in quantum mechanics. This will continue to be an important area of research in the future, as experimental techniques have advanced to a level that can be investigated at the atomic and molecular levels. The final two chapters track the progress of chaos into such diverse areas as biology and economics.

The book has been assembled out of the hard work of an international group of invited authors. It is a pleasure to thank them for their work and scientific contributions. I am grateful to acknowledge with much thanks the assistance provided by Ms. Sara Bacvarova, Author Service Manager, as well as the IntechOpen publishing group for the opportunity to work on this volume.

\section{Paul Bracken}

Professor, Department of Mathematics, University of Texas,

USA 

Section 1

\section{Chaos in Quantum Mechanics}





\title{
Introductory Chapter: Dynamical Symmetries and Quantum Chaos
}

\author{
Paul Bracken
}

\section{Introduction}

Chaos and many ideas from the study of this area have permeated a very large number of areas of science especially those which rely on mathematics. It is hoped this will illustrate how deeply and powerfully these ideas have influenced such areas as chemistry and physics.

Nature seems to be far too complicated to be linear at all levels all of the time. The exact laws of nature cannot be linear, nor can they be derived from such, to quote Einstein. Quantum mechanics, which is formally linear, is believed to be the underlying system to understand nature [1-3]. These seemingly conflicting views urge one to ask whether quantum mechanics can encompass nonlinear phenomena as well. This question is related to the study of classical nonlinear phenomena $[4,5]$. This leads one to wonder about the behavior of a quantum system if the classical version is chaotic. To understand chaos in quantum mechanics requires a more rigorous formulation of the fundamental structures of quantum theory $[6,7]$. To do this, one needs to formulate the quantum-classical correspondence, and at present, such a formulation is lacking.

In classical mechanics a Hamiltonian system with $N$ degrees of freedom is defined to be integrable if a set of $N$ constants of motion $\left\{F_{i}\right\}$ exist which are in involution, so the Poisson bracket satisfies $\left\{F_{i}, F_{j}\right\}=0$, with $i, j=1, \ldots, N$.

When the system is integrable, motion is restricted to an invariant $N$ torus in $2 N$-dimensional phase space and so is regular. If the system is perturbed by a small nonintegrable term, the Kolmogorov-Arnold-Moser (KAM) theorem states that its motion may still be restricted to the $N$-torus but be deformed. Chaos appears when such perturbations increase to such a degree that some tori are destroyed, and their behavior is characterized by positive Lyapunov exponents.

Attempts to investigate quantum chaos have focused on the quantization of classical nonintegrable systems. Since the former in principle is only a limiting case of the latter and most realistic quantum systems do not have a classical counterpart, the latter approach is more general and natural. The classical limit is most often approached by using Ehrenfest's theorem, and three popular ways to study the classical limit are given as follows. The Schrödinger approach is to develop a wave packet whose time evolution follows classical trajectories, so the time evolution of the coordinate and momentum expectation values solves not only Hamilton's equations but also Schrödinger's equation. Dirac's approach is to construct a quantum Poisson bracket such that the basic structure of classical and quantum mechanics is placed in one-to-one correspondence. The third approach is the Feynman path integral formalism, which expresses quantum mechanics in terms of classical concepts by integrating overall possible paths for a given initial and final state.

The problem may be reviewed based on the axiomatic structure of quantum mechanics, out of which the quantum dynamical degrees of freedom are defined 
and permit the construction of quantum phase space. This allows us to propose an idea for what quantum integrability is as well as its relationship with dynamical symmetry.

Quantum chaos is related to the question of the quantum-classical correspondence at two levels, kinematical and dynamical. The kinematical quantum-classical correspondence is a kind of reconciliation of the quantum and classical degrees of freedom and their associated geometrical structures.

Consistency of quantum theory implies there must exist a fundamental structure which can be used to determine the system's Hilbert space structure before solving the quantum dynamical equations. The axiomatic structure of quantum mechanics implies such a fundamental structure is simply the given algebraic structure of the system. The quantum mechanical Hilbert space is realized as a unitary irreducible representation of an algebra denoted as $\mathrm{g}$. For example, the harmonic oscillator is mentioned described by the Heisenberg algebra $h_{4}$ and specified by the operators $\left\{a, a^{\dagger}, a^{\dagger} a, I\right\}$. Here $a, a^{\dagger}$ are creation and annihilation operators for the oscillator. There is the spin system given by $s u(2)$ and spin operators $\left\{S_{-}, S_{+}, S_{0}\right\}$ and other systems such as the hydrogen and helium atoms. The associated covering group $G$ of $\mathbf{g}$ carries a natural geometric manifold, and all representations of quantum mechanics can be represented on such a geometrical manifold. Consequently, the kinematical correspondence can be constructed out of the dynamical group structure of the system, and the general solution is as follows.

A quantum system possesses a well-defined dynamical group $G$ over a Hilbert space $\mathcal{H}$. This can be regarded as an irrep space. The number of quantum dynamical degrees of freedom of the system is just the same number as the $M$ independent non-fully degenerate quantum numbers necessary to specify the space $\mathcal{H}$. The quantum phase space $\mathcal{P}$ is realized uniquely on a $2 M$ - dimensional coset space $G / H$ where $H \subset G$ is the maximal stability subgroup of a fixed state $\left|\psi_{0}\right\rangle \in \mathcal{H}$ of the system. The coset space $G / H$ and its global properties give a precise realization to the kinematical quantum-classical correspondence sought after. The fixed state $\left|\psi_{0}\right\rangle \in \mathcal{H}$ is the lowest (highest) weight state of $\mathcal{H}$ when $G$ is compact. When $G$ is a non-compact group, it is the lowest bound state of $\mathcal{H}$.

To see what can be extracted from this statement, consider now some nontrivial examples. In particular, let us clarify the idea of quantum dynamical degrees of freedom. The non-fully degenerate quantum numbers are defined by the nonconstant eigenvalues of a complete set of commuting operators in the associated basis.

The harmonic oscillator whose dynamical group is the Heisenberg-Weyl group $H_{4}$ and Hilbert space is the Fock space with $|n\rangle$ as its basis is specified by the non-fully degenerate quantum number $n$.

Next consider the spin system whose dynamical group is $S U(2)$. In its Hilbert space, a given irrep space of $S U(2)$ labeled $|j, m\rangle$, the total spin quantum number is a constant. The only non-fully degenerate quantum number to specify the basis is $m$, and the quantum dynamical degree of freedom is one, as its Hilbert space, which is an irrep space of $S U(2)$ often denoted as $\{|j, m\rangle\}$, has total quantum number one.

In the central potential problem, the dynamical group is $S U(1,1)$, and its quantum degree of freedom is one. The Hilbert space of $S U(1,1)$ is specified by two quantum numbers $k$ and $n$. The first is related to angular momentum, and the second is the principal quantum number. Since the angular momentum is conserved, the quantum number $k$ is fully degenerate.

The hydrogen atom and the relativistic free Dirac particle are perhaps the simplest and most realistic both having the dynamical group $S O(4,2)$. For hydrogen the three quantum dynamical degrees of freedom correspond to three non-fully 
degenerate quantum numbers, the principle quantum number $n$, angular momentum quantum number $j$, and $z$-component $m$. These label the Hilbert space completely, $\{|n, j, m\rangle\}$.

For the harmonic oscillator, to construct the phase space, the fixed state used is the vacuum state. The phase space is then $H_{4} / U(1) \otimes U(1)$, where $U(1) \otimes U(1)$ is invariant with respect to the vacuum. The phase space structure is determined by coherent state $|z\rangle$ of $H_{4} / U(1) \otimes U(1)$ :

$$
D(\alpha)|z\rangle=|z+\alpha\rangle, \quad D(\alpha) \in H_{4} / U(1) \otimes U(1) .
$$

The phase space is not complicated, just a one-dimensional complex space or two-dimensional real space.

For a spin system, the dynamical group is $S U(2)$, and in an irrep space $\{|j, m\rangle\}$, the fixed state is $|j,-j\rangle$. The phase space is $S U(2) / U(1)$, and is isomorphic to a twosphere, and there is the coherent state:

$$
|\Omega\rangle=D(z)|j, \quad-j\rangle=\exp \left(z S_{+}-z^{*} S_{-}\right)|j, \quad-j\rangle,
$$

where $s_{ \pm}$are spin raising and lowering operators. In a geometrical representation, $D(z)$ is

$$
\exp \left(\left(\begin{array}{ll}
0 & z \\
-z^{*} & 0
\end{array}\right)\right)=\left(\begin{array}{ll}
\cos |z| & \frac{z}{|z|} \sin |z| \\
-\frac{z^{*}}{|z|} \sin |z| & \cos |z|
\end{array}\right)=\left(\begin{array}{ll}
x_{0} & x \\
-x^{*} & x_{0}
\end{array}\right),
$$

where $x_{o}$ is real and $x=x_{1}+i x_{2}$. Unitarity of $D(z) \in S U(2) / U(1)$ requires $x_{o}^{2}+x_{1}^{2}+x_{2}^{2}=1$, which describes a two-sphere. The phase space is nontrivial, and canonical coordinates can be obtained from the coherent state basis as

$$
q=\sqrt{4 j \hbar} \sin \frac{\theta}{2} \cos \phi, \quad p=-\sqrt{4 j \hbar} \sin \frac{\theta}{2} \sin \phi .
$$

\section{Quenched quantum mechanics}

The dynamical correspondence of quantum-classical mechanics is a fundamental idea which should be addressed. In order to study the resultant singularity structures which result in a transition to chaos, it must be stated more precisely what this limit entails. Quenched quantum mechanics suggests a possible origin for a parameter which maps out this limit. Instead of considering $\hbar \rightarrow 0$, it involves allowing a parameter referred to as the quenching index $\tau$, which is dimensionless, to tend to infinity. In cases where $\tau$ turns out to be a fixed parameter, the system does not possess a classical limit. To understand properties of $\tau$, let us consider the case in which the associated Lie algebra splits up in the form $g=h \oplus k$ with $[h, h] \in h,[h, k] \in k,[k, k] \in h$, where $h$ is the Lie algebra of $H$ and the explicit form of $K(z, \bar{z})$ is

$$
K(z, \bar{z})=\operatorname{det}\left(I \pm Z^{\dagger} Z\right)^{ \pm \tau}
$$

In Eq. (3), $+(-)$ refers to the case where $G$ is compact (non-compact) and $Z$ a matrix with elements $z$ and $\tau$ is related to the matrix element $\left\langle 0\left|h_{i}\right| 0\right\rangle$ with $h_{i} \in h$. 
This gives rise to a geometrical interpretation for $\tau$. This can be seen by looking at the propagator on $G / H$.

To study quenched quantum mechanics, the propagator is expressed as

$$
U=\int D \mathbf{x} \exp \left(\frac{i}{\hbar} S\right)
$$

In Eq. (4), $D \mathbf{x}$ is the integration measure and $S$ is the effective action given as

$$
S=\theta-\mathcal{H} d t
$$

where $v$ is the one-form of $G / H$ and $\mathcal{H}$ the expectation value of the Hamiltonian operator $H=H\left(T_{i}\right), T_{i} \in G$, that is,

$$
V=i \frac{\hbar}{2}\left(\frac{\partial \ln K}{\partial z^{i}} d z^{i}-\frac{\partial \ln K}{\partial \bar{z}} d \bar{z}^{i}\right), \quad \mathcal{H}=\left\langle\Omega\left|H\left(T_{i}\right)\right| \Omega\right\rangle .
$$

The quantum equations depend on $\tau$. Upon expanding with respect to this parameter and not $\hbar$, the semi-quantal equations describing a classical-like system result. This arises from purely quantum structures and provides a way to achieve a classical limit:

$$
\lim _{Q \rightarrow \infty} \mathcal{H}=\mathcal{H}_{C}=H(\langle\Omega|H| \Omega\rangle)
$$

This limit may be divergent, since the phase space derived from the quantum geometry has not been scaled. Scaled canonical coordinates must be introduced to obtain a convergent limit as

$$
\frac{1}{\sqrt{2 \tau \hbar}}(q+i p)=\frac{Z}{\sqrt{I+Z^{\dagger} Z}}
$$

Expectation values of observables in coherent states can have correct dimensions in terms of $p$ and $q$ in such a coordinate system, and the semi-quantal dynamics in terms of $(p, q)$ in (7) is determined by the Hamilton equations:

$$
\frac{d q_{i}}{d t}=\frac{\partial(p, q)}{\partial p_{i}}, \quad \frac{d p_{i}}{d t}=-\frac{\partial \mathscr{H}}{\partial q_{i}}
$$

The difference between semi-quantal dynamics and classical mechanics is called the quantum fluctuation or correlation $\mathcal{H}-\mathcal{H}_{c}=f\left(z, z^{*}, \eta\right)$, which is clearly an explicit function of $\eta$. Once the quantum fluctuation is fixed in a quantum system, its dynamical evolution is determined by $\mathcal{H}$ not $\mathcal{H}_{c}$.

\section{Dynamical symmetry}

Let us discuss now some basic concepts related to chaos. One way to proceed is to study the behavior of quantum systems at the semi-quantal level by explicitly exploring the dynamical effects of quantum fluctuations on classical chaos. It would be good to find some general set of conditions which determine without great effort whether systems become chaotic and when.

The central idea of quantum integrability is dynamical symmetry. Integrability is a fundamental concept in the study of dynamical systems. Usually, the function 
of symmetry restricts the possible forms of Lagrangian, but not the associated dynamics.

A quantum system with dynamical group $G$ has a dynamical symmetry if and only if the Hamiltonian of the system can be written in terms of the Casimir operators of any particular subgroup chain $G^{\alpha}$ of $G: H=f\left(C_{k i}^{\alpha}\right)$, where $k=s^{\alpha}, \ldots, 1 ; i=1, \ldots, l_{k}^{\alpha}$. Here $\alpha$ is fixed and labels the particular subgroup chain, $C_{k i}^{\alpha}$ is the $\mathrm{i}$-th Casimir operator of subgroup $G_{k}^{\alpha}$ and $l_{k}^{\alpha}$ the rank of subgroup $G_{k}^{\alpha}$.

Dynamical symmetry is less restrictive on the system than pure, since the Hamiltonian and ground state are not necessarily invariant under a transformation of $G$. Quantum integrability can be formulated from the classical definition once dynamical degrees of freedom are specified and the quantum-classical correspondence of dynamics is elaborated. The Heisenberg equation, the quantum dynamical equation, has a similar structure to the classical dynamical system.

A quantum system with $M$ independent dynamical degrees of freedom and $2 M$ dimensional quantum phase space is integrable if and only if there are $M$ quantum constants of the motion or good quantum numbers. The corresponding constants of the motion have operators which commute with the Hamiltonian. From the definition of dynamical symmetry and quantum integrability, it can be shown that $a$ quantum system with a dynamical group $G$ is integrable if such a system possesses a dynamical symmetry of $G$.

Consider the example of an $N$-independent level system to illustrate the consistency of quantum and classical integrability. Introduce annihilation and creation operators for the state $|i\rangle$ such that $|i\rangle=b_{i}^{\dagger}|0\rangle,|0\rangle=b_{i}|i\rangle$ and $\left[b_{i}, b_{j}^{\dagger}\right]=\delta_{i j}$, $\left[b_{i}^{\dagger}, b_{j}^{\dagger}\right]=\left[b_{i}, b_{j}\right]=0$, Then the general form of the Hamiltonian is

$$
H=\sum_{i, j=1}^{n} H_{i j} b_{i}^{\dagger} b_{j} .
$$

The generators of the dynamical group $S U(N)$ are given as $E_{i j}=b_{i}^{\dagger} b_{j}$, and $H$ is a linear operator composed of the $E_{i j}$. From group theory, it is always possible to assume there is a $U(N)$ transformation such that $H=\tilde{H}=g g^{-1}$ where $g \in U(N)$ and

$$
\tilde{H}=\sum_{i=1}^{N} \tilde{H}_{i i} E_{i i} .
$$

It follows that $\tilde{H}$ and $H$ have the following dynamical symmetry

$$
U(N) \supset \ldots \supset C=U(1) \otimes U(1) \otimes \ldots \otimes U(1),
$$

where $C$ represents the Cartan subalgebra which is defined to be a product of $N$ factors of $U(1)$ with the generators $E_{i i}$. This implies the system is integrable. Consider now the phase space representation of this operator from quenched quantum mechanics, with phase space representation of $E_{i j}$ :

$$
\begin{gathered}
\mathcal{E}_{11}=N-\frac{1}{2}\left(p^{2}+q^{2}\right), \\
\mathcal{E}_{1 j}=\frac{1}{2}\left(q_{j}+i p_{j}\right) \sqrt{2 N-p^{2}-q^{2}} j \neq 1, \quad \mathcal{E}_{i j}=\frac{1}{2}\left(q_{j}+i p_{j}\right)\left(p_{i}+i q_{i}\right), \\
\mathcal{E}_{j i}=\left(\mathcal{E}_{i j}\right)^{\dagger}, \quad i, \quad j \neq 1 .
\end{gathered}
$$


where

$$
p^{2}+q^{2}=\sum_{i=2}^{N}\left(p_{i}^{2}+i q_{i}^{2}\right)
$$

The $\mathcal{E}_{i j}$ has the same algebraic structure as the $E_{i j}$, and the Hamiltonian function is only a decoupled function of $p_{i}^{2}+q_{i}^{2}$ in quadrature. All of this implies that the system is integrable, as might be expected on account of dynamical symmetry.

There is an important consequence of the results mentioned above.

Nonintegrability of a quantum system implies breaking of dynamical symmetry. This means that if chaos is present, dynamical symmetry of the system must be broken.

To develop this idea, if a system with $l$-rank, $n$-dimensional dynamical Lie group $G$ is nonautonomous, dynamical symmetry breaking implies the system becomes nonintegrable. For an autonomous system, the energy is conserved, and it becomes nonintegrable, and this means it is broken such that more than one of the $M \leq(n-l) / 2$ constants of motion are destroyed. It may be asked how much dynamical symmetry needs to be broken so that perturbative expansions about the dynamical symmetry basis will not converge.

Let us say that chaos will appear in a nonintegrable system when the breaking of the dynamical symmetry is accompanied by a structural phase transition. So if a structural phase transition takes place in a quantum system such that certain control parameters are altered, then it passes from one dynamical symmetry limit to another. Different dynamical symmetries connote different toroidal structures in $G / H$, so the torus structure must also alter from one to another. Consequently, dynamical symmetry breaking means that some or even all constants of motion are destroyed along with the corresponding tori giving rise to chaotic phenomenon.

Let us present a simple model which consists of two-spin coupled system governed by the Hamiltonian:

$$
H=(1-\alpha) \hbar\left(S_{1 z}+S_{2 z}\right)+\alpha h^{2} S_{1 x} S_{2 x}
$$

In (14), $\alpha$ is a coupling constant. This system has the possible dynamical symmetries:

$$
S U^{1}(2) \otimes S U^{2}(2) \supset\left\{\begin{array}{l}
S O^{1}(2) \otimes S O^{2}(2), \\
S U^{1+2}(2) \supset S O^{1+2}(2)
\end{array} .\right.
$$

The Hilbert space basis which carries the irreducible representations $\left(j_{1} j_{2}\right)$ of $S U^{1}(2) \otimes S U^{2}(2)$ are $\left\{\left|j_{1}, j_{2} ; m_{1}, m_{2}\right\rangle: m_{1}=-j_{1}, \ldots, j_{1}, m_{2}=-j_{2}, \ldots, j_{2}\right\}$ and $\left\{\left|j_{1}, j_{2} ; j, m\right\rangle, j=j_{1}+j_{2}, \ldots,\left|j_{1}-j_{2}\right|, m=-j \ldots j\right\}$ for the dynamical symmetry chains $(i)$ and $(i i)$.

The dynamical symmetries of $H$ are classified as: for $\alpha=0, H$ has symmetries (i) and (ii). For $\alpha=1$, the system is just in (i). However, when $0<\alpha<1$, dynamical symmetries are broken. The semi-quantal description can be used to see whether there has been a structural phase transition in the symmetry breaking phase. Coherent states are used to state the phase space is $S^{2} \otimes S^{2}$ and given as

$$
|p, q\rangle=\exp \sum_{i=1}^{2}\left(z_{i} J_{i+}-z_{i}^{*} J_{i-}\right)\left|j_{1}, j_{2} ;-j_{1},-j_{2}\right\rangle \text {. }
$$


where the canonical coordinates $(p, q)$ are given as

$$
\frac{1}{\sqrt{4 j_{i} \hbar}}\left(q_{i}+i p_{i}\right)=\frac{z_{i}}{\left|z_{i}\right|} \sin \left|z_{i}\right|=\sin \frac{\theta_{i}}{2} e^{-i \varphi_{i}}
$$

and $p_{i}^{2}+q_{i}^{2} \leq 4 j_{i} \hbar$. The Hamiltonian determined by semi-quantal dynamics is

$$
\begin{aligned}
\mathcal{H}=\langle p, q|H| p, q\rangle= & (1-\alpha)\left[\frac{1}{2}\left(p_{1}^{2}+q_{1}^{2}\right)+\frac{1}{2}\left(p_{2}^{2}+q_{2}^{2}\right)-\left(j_{1} \hbar+j_{2} \hbar\right)\right] \\
& +\frac{\alpha}{4} q_{1} q_{2} \sqrt{4 j_{1} \hbar-p_{1}^{2}-q_{1}^{2}} \sqrt{4 j_{2} \hbar-p_{2}^{2}-q_{2}^{2}} .
\end{aligned}
$$

Finally, it may be stated that to understand quantum chaos, one has to understand the dynamical behavior of a nonintegrable system when it deviates from the classical dynamics by taking into account nonvanishing quantum fluctuations. It may be asked whether the global phase space structure of classical dynamics can survive when quantum fluctuations are included. There is also the question of what governs the evolution of quantum fluctuations. It is required to have on hand a procedure which allows one to obtain the classical limit from a quantum system when one can only compute the deviations of the dynamics both close to and far from the classical limit. These deviations provide knowledge as to whether quantum fluctuations may alter classical dynamics and in what way. This is also deepening the understanding of the quantum-classical correspondence. Based on this, it may be asked whether the global phase space structure of classical mechanics can survive when quantum fluctuations are included and what actually governs the evolution of quantum fluctuations.

\section{Author details}

Paul Bracken

Department of Mathematics, University of Texas, Edinburg, TX, USA

*Address all correspondence to: paul.bracken@utrgv.edu

\section{IntechOpen}

(C) 2020 The Author(s). Licensee IntechOpen. This chapter is distributed under the terms of the Creative Commons Attribution License (http://creativecommons.org/licenses/ by/3.0), which permits unrestricted use, distribution, and reproduction in any medium, provided the original work is properly cited. (cc) BY 


\section{References}

[1] Blümel GL, Reinhardt WP. Chaos in Atomic Physics. Cambridge: Cambridge University Press; 1997

[2] Baker GL, Gollub JP. Chaotic Dynamics. Cambridge: Cambridge University Press; 1990

[3] Berry MV. Quantum chaology, not quantum chaos. Physica Scripta. 1989; 40:335-336

[4] Devaney RL. A First Coarse in Chaotic Dynamical Systems. Reading, MA: Addison-Wesley; 1992

[5] Ott E. Chaos in Dynamical Systems. Cambridge: Cambridge University Press; 1993

[6] Dirac PAM. The adiabatic invariance of the quantum integrals. Proceedings of the Royal Society. 1925;107:725-734

[7] Zhang W-M, Feng DH, Yuan J-M, Wong S-J. Integrability and nonintegrability of quantum systems: Quantum integrability and dynamical symmetry. Physical Review A. 1989;40: 438-446 
Section 2

Nonlinear Dynamics 



\title{
Chapter 2
}

\section{Loop-like Solitons}

\author{
Vyacheslav O. Vakhnenko, E. John Parkes and \\ Dmitri B. Vengrovich
}

\begin{abstract}
The physical phenomena that take place in nature generally have complicated nonlinear features. A variety of methods for examining the properties and solutions of nonlinear evolution equations are explored by using the Vakhnenko equation (VE) as an example. One remarkable feature of the VE is that it possesses loop-like soliton solutions. Loop-like solitons are a class of interesting wave phenomena, which have been involved in some nonlinear systems. The VE can be written in an alternative form, known as the Vakhnenko-Parkes equation (VPE). The VPE can be written in Hirota bilinear form. The Hirota method not only gives the $N$-soliton solution but enables one to find a way from the Bäcklund transformation through the conservation laws and associated eigenvalue problem to the inverse scattering transform (IST) method. This method is the most appropriate way of tackling the initial value problem (Cauchy problem). The standard procedure for IST method is expanded for the case of multiple poles, specifically, for the double poles with a single pole. In recent papers some physical phenomena in optics and magnetism are satisfactorily described by means of the VE. The question of physical interpretation of multivalued (loop-like) solutions is still an open question.
\end{abstract}

Keywords: nonlinear evolution equations, solutions, Vakhnenko equation, Hirota method, Bäcklund transformation, inverse scattering problem, $N$-soliton solution, spectral data

PACS: 00.30.Lk 02.30.Jr, 05.45.Yv

\section{The high-frequency perturbations in a relaxing medium}

From the nonequilibrium thermodynamic standpoint, models of a relaxing medium are more general than equilibrium models. To develop physical models for wave propagation through media with complicated inner kinetics, notions based on the relaxational nature of a phenomenon are regarded to be promising. Thermodynamic equilibrium is disturbed owing to the propagation of fast perturbations. There are processes of the interaction that tend to return the equilibrium. The parameters characterizing this interaction are referred to as the inner variables unlike the macroparameters such as the pressure $p$, mass velocity $u$ and density $\rho$. In essence, the change of macroparameters caused by the changes of inner parameters is a relaxation process. 
We restrict our attention to barotropic media. An equilibrium state equation of a barotropic medium is a one-parameter equation. As a result of relaxation, an additional variable $\xi$ (the inner parameter) appears in the state equation

$$
p=p(\rho, \xi)
$$

and defines the completeness of the relaxation process. There are two limiting cases with corresponding sound velocities:

i. Lack of relaxation (inner interaction processes are frozen) for which $\xi=1$ :

$$
p=p(\rho, 1) \equiv p_{f}(\rho), \quad c_{f}^{2}=d p_{f} / d \rho ;
$$

ii. Relaxation which is complete (there is local thermodynamic equilibrium) for which $\xi=0$ :

$$
p=p(\rho, 0) \equiv p_{e}(\rho), \quad c_{e}^{2}=d p_{e} / d \rho
$$

Slow and fast processes are compared by means of the relaxation time $\tau_{p}$.

To analyse the wave motion, we use the following hydrodynamic equations in Lagrangian coordinates:

$$
\frac{\partial V}{\partial t}-\frac{1}{\rho_{0}} \frac{\partial u}{\partial x}=0, \quad \frac{\partial u}{\partial t}+\frac{1}{\rho_{0}} \frac{\partial p}{\partial x}=0 .
$$

The following dynamic state equation is applied to account for the relaxation effects:

$$
\tau_{p}\left(\frac{d p}{d t}-c_{f}^{2} \frac{d \rho}{d t}\right)+\left(p-p_{e}\right)=0
$$

Here $V \equiv \rho^{-1}$ is the specific volume and $x$ is the Lagrangian space coordinate. Clearly, for the fast processes $\left(\omega \tau_{p} \gg 1\right)$, we have relation (2), and for the slow ones $\left(\omega \tau_{p} \ll 1\right)$, we have (3).

The closed system of equations consists of two motion equations (4) and dynamic state equation (5). The motion equations (4) are written in Lagrangian coordinates since the state equation (5) is related to the element of mass of the medium.

The substantiation of (5) within the framework of the thermodynamics of irreversible processes has been given in $[1,2]$. We note that the mechanisms of the exchange processes are not defined concretely when deriving the dynamic state equation (5). In this equation the thermodynamic and kinetic parameters appear only as sound velocities $c_{e}$ and $c_{f}$ and relaxation time $\tau_{p}$. These are very common characteristics and they can be found experimentally. Hence, it is not necessary to know the inner exchange mechanism in detail.

Combining the relationships (4) and (5), we obtain for low-frequency perturbations $\left(\tau_{p} \omega \ll 1\right)$ the Korteweg-de Vries-Burgers (KdVB) equation:

$$
\begin{aligned}
& \frac{\partial p}{\partial t}+c_{e} \frac{\partial p}{\partial x}+\alpha_{e} c_{e}^{3} p \frac{\partial p}{\partial x}-\beta_{e} \frac{\partial^{2} p}{\partial x^{2}}+\gamma_{e} \frac{\partial^{3} p}{\partial x^{3}}=0, \\
& \beta_{e}=\frac{c_{e}^{2} \tau_{p}}{2 c_{f}^{2}}\left(c_{f}^{2}-c_{e}^{2}\right), \quad \gamma_{e}=\frac{c_{e}^{3} \tau_{p}^{2}}{8 c_{f}^{4}}\left(c_{f}^{2}-c_{e}^{2}\right)\left(c_{f}^{2}-5 c_{e}^{2}\right),
\end{aligned}
$$

whilst for high-frequency waves $\left(\tau_{p} \omega \gg 1\right)$, we have obtained the following equation: 


$$
\begin{aligned}
& \frac{\partial^{2} p}{\partial x^{2}}-c_{f}^{-2} \frac{\partial^{2} p}{\partial t^{2}}+\alpha_{f} c_{f}^{2} \frac{\partial^{2} p^{2}}{\partial x^{2}}+\beta_{f} \frac{\partial p}{\partial x}+\gamma_{f} p=0, \\
& \beta_{f}=\frac{c_{f}^{2}-c_{e}^{2}}{\tau_{p} c_{e}^{2} c_{f}}, \quad \gamma_{f}=\frac{c_{f}^{4}-c_{e}^{4}}{2 \tau_{p}^{2} c_{e}^{4} c_{f}^{2}} .
\end{aligned}
$$

Equation (6) with $\left(\beta_{e}=0\right)$ is the well-known the Korteweg-de Vries (KdV) equation. The investigation of the $\mathrm{KdV}$ equation in conjunction with the nonlinear Schrodinger (NLS) and sine-Gordon equations gives rise to the theory of solitons [4-13].

We focus our main attention on (7). It has a dissipative term $\beta_{f} \partial p / \partial x$ and a dispersive term $\gamma_{f} p$. Without the nonlinear and dissipative terms, we have a linear Klein-Gordon equation.

Let us write down (7) in dimensionless form. In the moving coordinate system with velocity $c_{f}$, after factorization the equation has the form in the dimensionless variables $\tilde{x}=\sqrt{\frac{\gamma_{f}}{2}}\left(x-c_{f} t\right), \tilde{t}=\sqrt{\frac{\gamma_{f}}{2}} c_{f} t, \tilde{u}=\alpha_{f} c_{f}^{2} p$ (tilde over variables $\tilde{x}, \tilde{t}$ and $\tilde{u}$ is omitted)

$$
\frac{\partial}{\partial x}\left(\frac{\partial}{\partial t}+u \frac{\partial}{\partial x}\right) u+\alpha \frac{\partial u}{\partial x}+u=0
$$

The constant $\alpha=\beta_{f} / \sqrt{2 \gamma_{f}}$ is always positive. Equation (8) without the dissipative term has the form of the nonlinear equation $[14,15]$ :

$$
\frac{\partial}{\partial x}\left(\frac{\partial}{\partial t}+u \frac{\partial}{\partial x}\right) u+u=0
$$

Historically, (9) has been called the Vakhnenko equation (VE), and we will follow this name.

We note that (9) follows as a particular limit of the following generalized Korteweg-de Vries equation:

$$
\frac{\partial}{\partial x}\left(\frac{\partial u}{\partial t}+u \frac{\partial u}{\partial x}-\beta \frac{\partial^{3} u}{\partial x^{3}}\right)=\gamma u
$$

derived by Ostrovsky [16] to model small-amplitude long waves in a rotating fluid ( $\gamma u$ is induced by the Coriolis force) of finite depth. Subsequently, (9) was known by different names in the literature, such as the Ostrovsky-Hunter equation, the short-wave equation, the reduced Ostrovsky equation, and the OstrovskyVakhnenko equation depending on the physical context in which it is studied.

The consideration here of (9) has interest from the viewpoint of the investigation of the propagation of high-frequency perturbations.

\section{Loop-like stationary solutions}

The travelling wave solutions are solutions which are stationary with respect to a moving frame of reference. In this case, the evolution equation (a partial differential equation) becomes an ordinary differential equation (ODE) which is considerably easier to solve.

For the VE (9) it is convenient to introduce a new dependent variable $z$ and new independent variables $\eta$ and $\tau$ defined by

$$
z=(u-v) /|v|, \quad \eta=(x-v t) /|v|^{1 / 2}, \quad \tau=t|v|^{1 / 2},
$$


where $v$ is a nonzero constant [15]. Then the VE becomes begin equation:

$$
z_{\eta \tau}+\left(z z_{\eta}\right)_{\eta}+z+c=0
$$

where $c= \pm 1$ corresponding to $v \gtrless 0$. We now seek stationary solutions of (12) for which $z$ is a function of $\eta$ only so that $z_{\tau}=0$ and $z$ satisfies the ODE:

$$
\left(z z_{\eta}\right)_{\eta}+z+c=0
$$

After one integration (13) gives

$$
\begin{aligned}
\frac{1}{2}\left(z z_{\eta}\right)^{2} & =f(z), \\
f(z) & =-\frac{1}{3} z^{3}-\frac{1}{2} c z^{2}+\frac{1}{6} A=-\frac{1}{3}\left(z-z_{1}\right)\left(z-z_{2}\right)\left(z-z_{3}\right) .
\end{aligned}
$$

where $A$ is a constant, and for periodic solutions $z_{1}, z_{2}$ and $z_{3}$ are real constants such that $z_{1} \leq z_{2} \leq z_{3}$. On using results 236.00 and 236.01 of [17], we may integrate (14) to obtain

$$
\begin{gathered}
\eta=\frac{\sqrt{6} z_{1}}{\sqrt{z_{3}-z_{1}}} F(\varphi, m)+\sqrt{6\left(z_{3}-z_{1}\right)} E(\varphi, m), \\
\sin \varphi=\frac{z_{3}-z}{z_{3}-z_{2}}, \quad m=\frac{z_{3}-z_{2}}{z_{3}-z_{1}} .
\end{gathered}
$$

where $F(\varphi, m)$ and $E(\varphi, m)$ are incomplete elliptic integrals of the first and second kind, respectively. We have chosen the constant of integration in (15) to be zero so that $z=z_{3}$ at $\eta=0$. The relations (15) give the required solution in parametric form, with $z$ and $\eta$ as functions of the parameter $\varphi$.

For $c=1$ (i.e., $v>0$ ), there are periodic solutions for $0<A<1$ with $\lambda<0$, $z_{2} \in(-1,0)$ and $z_{3} \in(0,0.5)$; an example of such a periodic wave is illustrated by curve 2 in Figure 1. Here we introduce a new independent variable $\zeta$ defined by

$$
\frac{d \eta}{d \zeta}=z
$$

$A=1$ gives the solitary wave limit:

$$
u=\frac{3}{2} v \operatorname{sech}^{2}(\zeta / 2), \quad \eta=-\zeta+3 \tanh (\zeta / 2)
$$

as illustrated by curve 1 in Figure 1 . The periodic waves and the solitary wave have a loop-like structure as illustrated in Figure 1. For $c=-1$ (i.e., $v<0$ ), there are periodic waves for $-1<A<0$ with $\lambda>0, z_{2} \in(0,1)$ and $z_{3} \in(1,1.5)$; an example of such a periodic wave is illustrated by curve 2 in Figure 2 . When $A=0$ and $\lambda=6$, then the periodic wave solution simplifies to

$$
u(\eta) /|v|=-\frac{1}{6} \eta^{2}+\frac{1}{2}, \quad-3 \leq \eta \leq 3, \quad u(\eta+6)=u(\eta) .
$$

This is shown by curve 1 in Figure 2. For $A \simeq-1$ the solution has a sinusoidal form (curve 3 in Figure 2). Note that there are no solitary wave solutions.

A remarkable feature of the equation (9) is that it has a solitary wave (18) which has a loop-like form, i.e., it is a multivalued function (see Figure 1). Whilst loop 


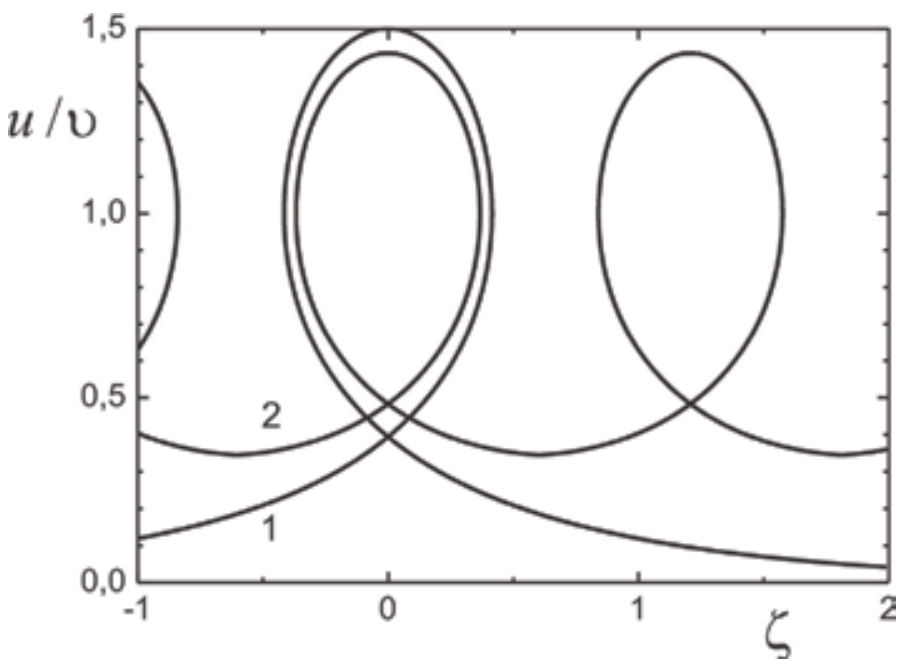

Figure 1.

Travelling wave solutions with $v>0$.

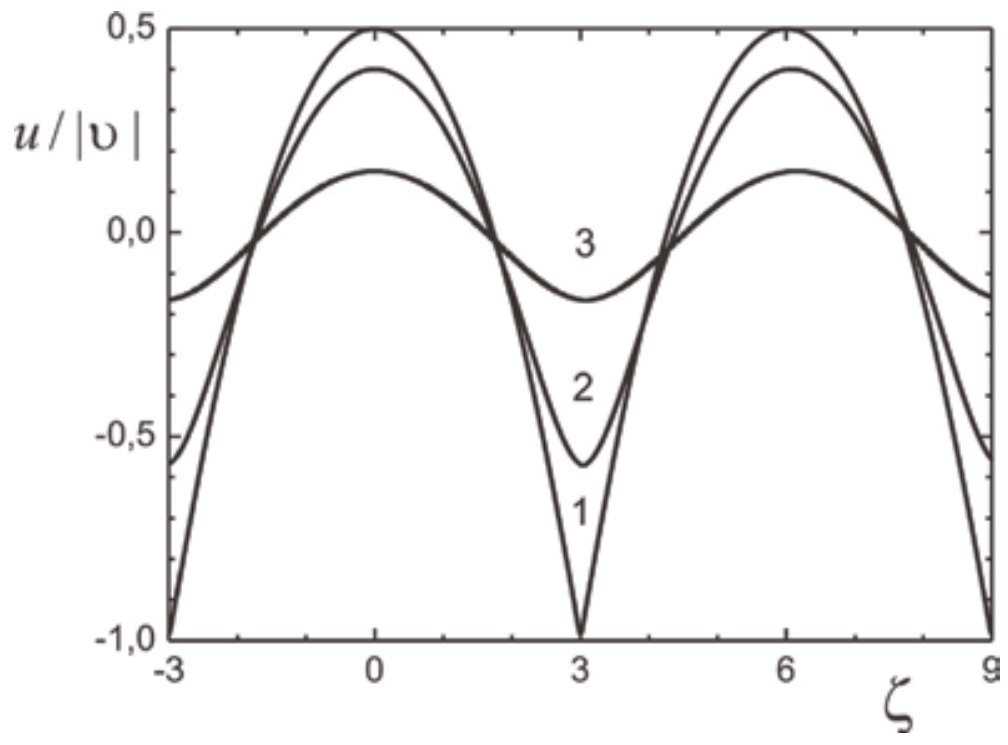

Figure 2.

Travelling wave solutions with $v<0$.

solitary waves (18) are rather intriguing, it is the solution to the initial value problem that is of more interest in a physical context. An important question is the stability of the loop-like solutions. Although the analysis of stability does not link with the theory of solitons directly, the method applied in [15] is instructive, since it is successful in a nonlinear approximation. Stability of the loop-like solutions has been proved in [15]. From a physical viewpoint, the stability or otherwise of solutions is essential to their interpretation.

\section{The Vakhnenko-Parkes equation}

The multivalued solutions obtained in Section 2 obviously mean that the study of the VE $(9)$ in the original coordinates $(x, t)$ leads to certain difficulties. 
These difficulties can be avoided by writing down the VE in new independent coordinates. We have succeeded in finding these coordinates. Historically, working separately, we (Vyacheslav Vakhnenko in Ukraine and John Parkes in the UK) independently suggested such independent coordinates in which the solutions become one-valued functions. It is instructive to present the two derivations here. In one derivation a physical approach, namely, a transformation between Euler and Lagrange coordinates, was used, whereas in the other derivation, a pure mathematical approach was used.

Let us define new independent variables $(X, T)$ by the transformation

$$
\varphi d T=d x-u d t, \quad X=t .
$$

The function $\varphi$ is to be obtained. It is important that the functions $x=\theta(X, T)$ and $u=U(X, T)$ turn out to be single-valued. In terms of the coordinates $(X, T)$, the solution of the VE (9) is given by single-valued parametric relations. The transformation into these coordinates is the key point in solving the problem of the interaction of solitons as well as explaining the multivalued solutions [3]. The transformation (20) is similar to the transformation between Eulerian coordinates $(x, t)$ and Lagrangian coordinates $(X, T)$. We require that $T=x$ if there is no perturbation, i.e., if $u(x, t) \equiv 0$. Hence $\varphi=1$ when $u(x, t) \equiv 0$.

The function $\varphi$ is the additional dependent variable in the equation system (22), (24) to which we reduce the original Eq. (9). We note that the transformation inverse to (20) is

$$
d x=\varphi d T+U d X, \quad t=X, \quad U(X, T) \equiv u(x, t) .
$$

It follows that

$$
\frac{\partial x}{\partial X}=U, \quad \frac{\partial x}{\partial T}=\varphi, \quad \frac{\partial t}{\partial X}=1, \quad \frac{\partial t}{\partial T}=0
$$

Hence

$$
\frac{\partial \varphi}{\partial X}=\frac{\partial U}{\partial T}
$$

and

$$
\frac{\partial}{\partial X}=\frac{\partial}{\partial t}+u \frac{\partial}{\partial x}, \quad \frac{\partial}{\partial T}=\varphi \frac{\partial}{\partial x} .
$$

By using (23), we can write Eq. (9) in terms of $\varphi(X, T)$ and $U(X, T)$, namely,

$$
U_{X T}+\varphi U=0 .
$$

Equations (22) and (24) are the main system of equations. It can be reduced to a nonlinear equation (27) in one unknown $W$ defined by

$$
W_{X}=U
$$

From (22), (25) and the requirement that $\varphi=1$ when $U \equiv 0$, we have

$$
\varphi=1+W_{T} .
$$

Then, by eliminating $\varphi$ and $U$ between (24), (25) and (26), we arrive at a transformed form of the VE (9), namely, 


$$
W_{X X T}+\left(1+W_{T}\right) W_{X}=0 .
$$

Alternatively, by eliminating $\varphi$ between (22) and (24), we obtain

$$
U U_{X X T}-U_{X} U_{X T}+U^{2} U_{T}=0 .
$$

Furthermore it follows from (21) that the original independent coordinates $(x, t)$ are given by

$$
x=\theta(X, T)=x_{0}+T+W, \quad t=X,
$$

where $x_{0}$ is an arbitrary constant. Since the functions $\theta(X, T)$ and $U(X, T)$ are single-valued, the problem of multivalued solutions has been resolved from the mathematical point of view.

Alternatively, in a pure mathematical approach, we may start by introducing new independent variables $X$ and $T$ defined by

$$
x=T+\int_{-\infty}^{X} U\left(X^{\prime}, T\right) d X^{\prime}+x_{1}, \quad t=X,
$$

where $x_{1}$ is an arbitrary constant. From (30), we obtain (23) but with

$$
\varphi(X, T)=1+\int_{-\infty}^{X} U_{T} d X^{\prime} .
$$

Now, on introducing (25), (30) and (31) may be identified with (29) and (26), respectively. The derivation of (27) and (28) proceeds as before.

The transformation into new coordinates, as has already been pointed out, was obtained by us independently of each other; nevertheless, we published the result together $[18,19]$. Following the papers [20-23] hereafter, Eq. (27) (or in alternative form (28)) is referred to as the Vakhnenko-Parkes equation (VPE).

The travelling wave solution (15) and (16) for Equation (9) is also a travelling wave solution when written in terms of the transformed coordinates $(X, T)$. In order to do this, we need to express the independent variable $\zeta$, as introduced in (17), in terms of $X$ and $T$.

From the expressions for $z$ in (11) and (17), we obtain

$$
\frac{d \eta}{d \zeta}=\frac{U-v}{|v|}
$$

so that

$$
|v| \eta=\int U d \zeta-v \zeta
$$

From the definition of $\eta$ in (17), and the expressions for $x$ and $t$ given by (29), we obtain

$$
|v| \eta=|v|^{1 / 2}[W-v(X-V T)], \quad \text { where } \quad V:=v^{-1} .
$$

The expressions for $|v| \eta$ in (33) and (34) are equivalent if

$$
\zeta=|v|^{1 / 2} Z, \quad \text { where } \quad Z:=X-V T-X_{0}
$$


and $X_{0}$ is an arbitrary constant, so that

$$
W=\int U d Z \text { and } U=W_{Z}
$$

Hence, from (34), it follows that

$$
W=\frac{\sqrt{|v|}}{p}\left[\left(z_{1}+c\right) w+\left(z_{3}-z_{1}\right) E(w \mid m)\right]+W_{0},
$$

where $w=p \sqrt{|v|} Z$ and $W_{0}$ is an arbitrary constant. Then

$$
\frac{U}{|v|}=c+z_{3}-\left(z_{3}-z_{2}\right) \operatorname{sn}^{2}(w \mid m), \text { where } w=p \sqrt{|v|} Z .
$$

Eqs. (37) and (38) give the travelling wave solutions to the VPE in the forms (27) and (28), respectively. Eq. (38) is also the travelling wave solution of the VE (9) expressed in terms of the new coordinates $(X, T)$. In the limiting case $m=1$, (38) gives a solitary wave in the following two forms: For $v>0$

$$
U / v=\frac{3}{2} \operatorname{sech}^{2}\left(\frac{1}{2} \sqrt{v} Z\right)
$$

and, for $v<0$,

$$
U /|v|=-1+\frac{3}{2} \operatorname{sech}^{2}\left(\frac{1}{2} \sqrt{|v| Z}\right)
$$

These two solutions are illustrated by curve 1 in Figures 3 and 4, respectively. The other curves illustrate examples of the solution given by (38) when $m \neq 1$. Curves 1 and 2 in Figure 3 relate to curves 1 and 2, respectively, in Figure 1. Curves 1, 2 and 3 in Figure 4 relate to curves 1, 2 and 3, respectively, in Figure 2.

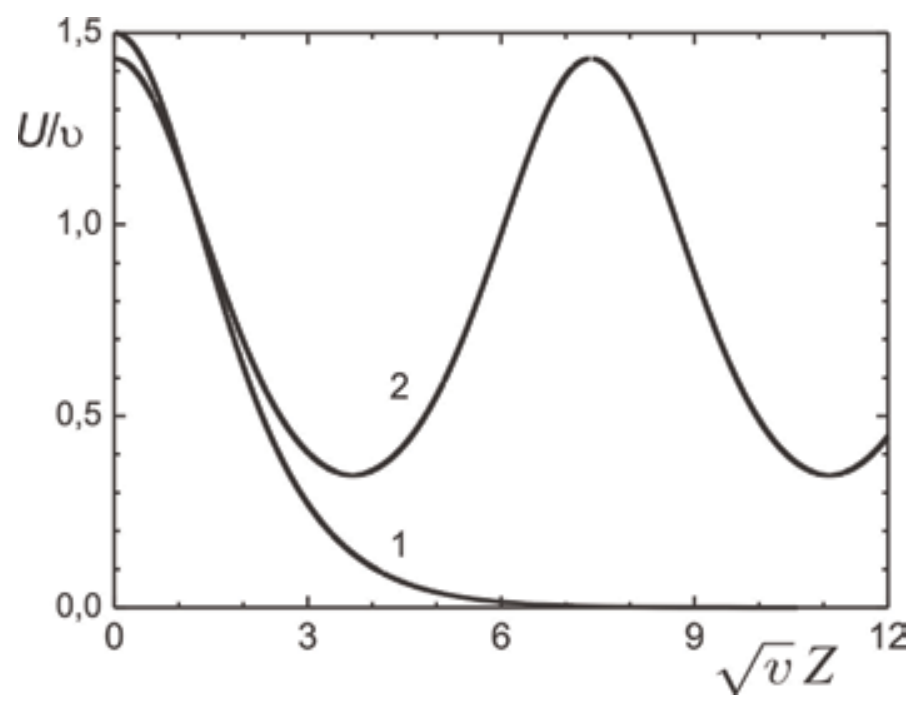

Figure 3.

Travelling wave solutions with $v>0$ in coordinates $(X, T)$. 


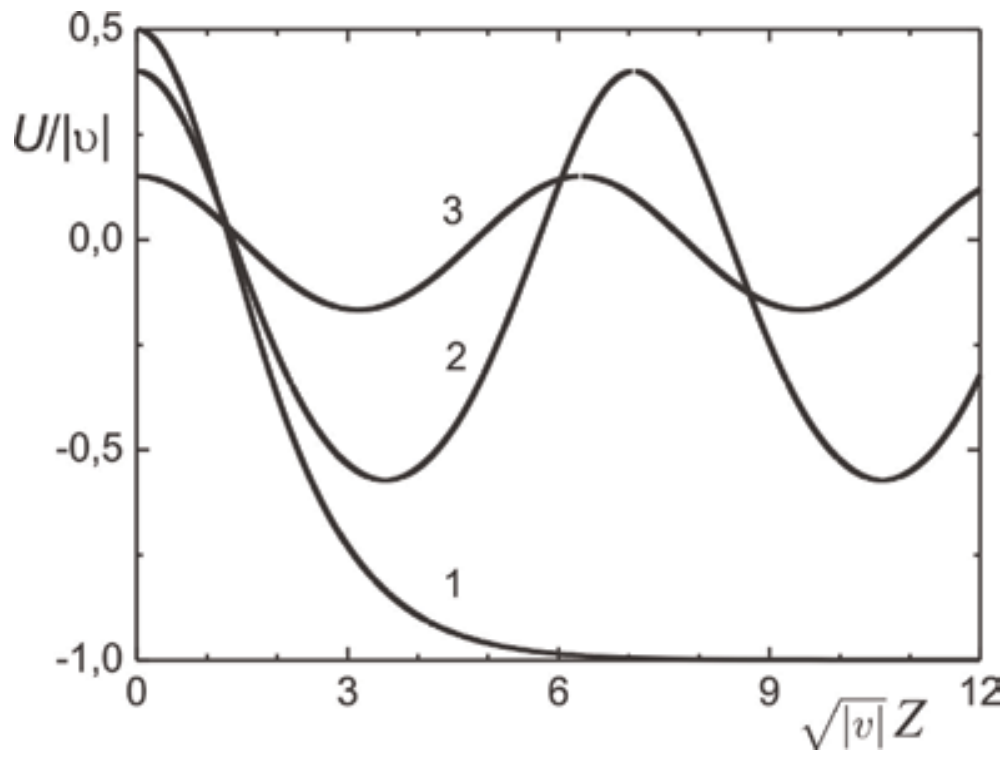

Figure 4.

Travelling wave solutions with $v<0$ in coordinates $(X, T)$.

There are two important observations to be made. Firstly, all the travelling wave solutions in terms of the new coordinates are single-valued. Secondly, the periodic solution shown by curve 1 in Figure 2, i.e., the solution consisting of parabolas, is not periodic in terms of the new coordinates. Hence, we reveal some accordance between curve 1 in Figure 3 and curve 1 in Figure 4. These features are important for finding the solutions by the inverse scattering method [24-30].

\section{From Hirota method to the inverse scattering method}

The Hirota method gives the $\mathrm{N}$-soliton solution as well as enables one to find a way from the Bäcklund transformation through the conservation laws and associated eigenvalue problem to the inverse scattering method [24]. Thus, the Hirota method allows us to formulate the inverse scattering method which is the most appropriate way of tackling the initial value problem (Cauchy problem).

In the Hirota method the equation, in our case the VPE (27), under investigation should be transformed into the Hirota bilinear form $[9,24]$ :

$$
\left(D_{T} D_{X}^{3}+D_{X}^{2}\right) f \cdot f=0
$$

with

$$
W=6(\ln f)_{X},
$$

The Hirota bilinear D-operator is defined as (see Section 5.2 in [9])

$$
D_{T}^{n} D_{X}^{m} a \cdot b=\left.\left(\frac{\partial}{\partial T}-\frac{\partial}{\partial T^{\prime}}\right)^{n}\left(\frac{\partial}{\partial X}-\frac{\partial}{\partial X^{\prime}}\right)^{m} a(T, X) b\left(T^{\prime}, X^{\prime}\right)\right|_{T=T^{\prime}, X=X^{\prime}} .
$$

Now we present a Bäcklund transformation for VPE (27) written in the bilinear form (41). This type of Bäcklund transformation was first introduced by Hirota [31] 
and has the advantage that the transformation equations are linear with respect to each dependent variable. This Bäcklund transformation can be transformed to the ordinary one [24]:

$$
\begin{gathered}
\left(D_{X}^{3}-\lambda\right) f^{\prime} \cdot f=0, \\
\left(3 D_{X} D_{T}+1+\mu D_{X}\right) f^{\prime} \cdot f=0,
\end{gathered}
$$

where $\lambda=\lambda(X)$ is an arbitrary function of $X$ and $\mu=\mu(T)$ is an arbitrary function of $T$.

The inverse scattering transform (IST) method is arguably the most important discovery in the theory of solitons. The method enables one to solve the initial value problem for a nonlinear evolution equation. Moreover, it provides a proof of the complete integrability of the equation.

The essence of the application of the IST is as follows. The initial equation VPE (27) is written as the compatibility condition for two linear equations. These equations are presented in (47) and (48). Then $W(X, 0)$ is mapped into the scattering $S(0)$ for (47). It is important that since the variable $W(X, T)$ contained in the spectral equation (47) evolves according to (27), the spectrum $\lambda$ always retains constant values. The time evolution of $S(T)$ is simple and linear. From a knowledge of $S(T)$, we reconstruct $W(X, T)$.

The use of the IST is the most appropriate way of tackling the initial value problem. In order to apply the IST method, one first has to formulate the associated eigenvalue problem. This can be achieved by finding a Bäcklund transformation associated with the VPE.

Now we will show that the IST problem for the VPE in the form (27) has a thirdorder eigenvalue problem that is similar to the one associated with a higher-order $\mathrm{KdV}$ equation [32, 33], a Boussinesq equation [33-37] and a model equation for shallow water waves $[9,38]$.

Introducing the function

$$
\psi=f^{\prime} / f
$$

and taking into account (42), we find that (44) and (45) reduce to

$$
\begin{gathered}
\psi_{X X X}+W_{X} \psi_{X}-\lambda \psi=0, \\
3 \psi_{X T}+\left(1+W_{T}\right) \psi+\mu \psi_{X}=0,
\end{gathered}
$$

respectively, where we have used results similar to (X.1)-(X.3) in [9]. From (47) and (48), it can be shown that

$$
3 \lambda \psi_{T}+\left(1+W_{T}\right) \psi_{X X}-W_{X T} \psi_{X}+\left[W_{X X T}+\left(1+W_{T}\right) W_{X}+\mu \lambda\right] \psi=0
$$

and

$$
\left[W_{X X T}+\left(1+W_{T}\right) W_{X}\right]_{X} \psi+\left(3 \psi_{T}+\mu \psi\right) \lambda_{X}=0 .
$$

In view of (27), (49) becomes

$$
3 \lambda \psi_{T}+\left(1+W_{T}\right) \psi_{X X}-W_{X T} \psi_{X}+\lambda \mu \psi=0,
$$

and (50) implies that $\lambda_{X}=0$ so the spectrum $\lambda$ of (47) remains constant. Constant $\lambda$ is what is required in the IST problem. Equation (50) yields the equation $W_{X X T}+\left(1+W_{T}\right) W_{X}=h(T)$, where $h(T)$ is an arbitrary function of $T$. 
Now, according to (62) and (72), the inverse scattering method restricts the solutions to those that vanish as $|X| \rightarrow \infty$, so $h(T)$ is to be identically zero. Thus, the pair of equations (47) and (48) or (47) and (50) can be considered as the Lax pair for the VPE (27).

Since (47) and (48) are alternative forms of Eqs. (44) and (45), respectively, it follows that the pair of equations (47) and (48) is associated with the VPE (27) considered here. Thus, the IST problem is directly related to a spectral equation of third order, namely, (47). The inverse problem for certain third-order spectral equations has been considered by Kaup [33] and Caudrey [34, 35]. As expected, (47) and (48) are similar to, but cannot be transformed into, the corresponding equations for the Hirota-Satsuma equation (HSE) (see Eq. (A8a) and (A8b) in [39]). Clarkson and Mansfield [40] note that the scattering problem for the HSE is similar to that for the Boussinesq equation which has been studied comprehensively by Deift et al. [37].

\section{The inverse scattering method for a third-order equation}

\subsection{Example of the use of the IST method to find the one-soliton solution}

Consider the one-soliton solution of the VPE by application of the IST method. Let the initial perturbation be

$$
W(X, 0)=6 k(1+\tanh (\eta)), \quad \eta=k X+\alpha .
$$

For convenience we introduce new notation $\xi_{1}$ and $\beta_{1}$ instead of parameters $k$ and $\alpha$ by

$$
k=\frac{\sqrt{3}}{2} \xi_{1}, \quad \alpha=\frac{1}{2} \ln \left(\beta_{1} / 2 \sqrt{3} \xi_{1}\right)
$$

then

$$
W(X, 0)=6 \sqrt{3} \xi_{1} \frac{\partial}{\partial X} \ln \left[1+\frac{\beta_{1}}{2 \sqrt{3} \xi_{1}} \exp \left(\sqrt{3} \xi_{1} X\right)\right]
$$

is the initial condition for the VPE.

The first step in the IST method is to solve the spectral equation (47) with spectral parameter $\lambda$ for the given initial condition $W(X, 0)$. In our example it is (54). The solution is studied over the complex $\zeta$-plane, where $\zeta^{3}=\lambda$. One can verify by direct substitution of (55) in (47) that the solution $\psi(X, 0 ; \zeta)$ of the linear ODE (47), normalized so that $\psi(X, 0 ; \zeta) \exp (-\zeta X) \rightarrow 1$ at $X \rightarrow-\infty$, is given by

$$
\psi(X, 0 ; \zeta) \exp (-\zeta X)=1-\frac{\beta_{1} \exp \left(\sqrt{3} \xi_{1} X\right)}{1+\beta_{1} \frac{\exp \left(\sqrt{3} \xi_{1} X\right)}{2 \sqrt{3} \xi_{1}}}\left[\frac{\omega_{2}}{\mathrm{i} \omega_{2} \xi_{1}-\zeta}+\frac{\omega_{3}}{-\mathrm{i} \omega_{3} \xi_{1}-\zeta}\right]
$$

where $\omega_{j}=e^{\mathrm{i} 2 \pi(j-1) / 3}$ are the cube roots of $1(j=1,2,3)$. The constants $\beta_{1}$ and $\xi_{1}$, as we will show, are associated with the local spectral data.

The second step in the IST method is to obtain the evolution of $\beta_{1}$ and $\xi_{1}$. The time dependence of the solution $\psi(X, T)$ is described by Eq. (48). Analysing Eq. (48), we may assume that 


$$
\begin{aligned}
& \xi_{1}(T)=\xi_{1}(0)=\text { const., } \\
& \beta_{1}(T)=\beta_{1}(0) \exp \left(-\frac{1}{\sqrt{3} \xi_{1}} T\right)
\end{aligned}
$$

Below, the assumption of these relationships will be justified. Indeed, we know that the spectrum $\lambda$ in (47) remains constant if $W(X, T)$ evolves according to Eq. (27). Therefore, as will be proved, the spectrum data evolve as in (70). In notations (77) and (78), from (70) we obtain the relations (56).

The final step in IST method is to select the solution $W(X, T)$ from (55) with $\xi_{1}(T), \beta_{1}(T)$ as in (56). According to Eq. (2.7) in [33], we expand $\psi(X, T ; \zeta)$ as an asymptotic series in $\zeta^{-1}$ to obtain

$$
\psi(X, 0 ; \zeta) \exp (-\zeta X)=1-\frac{1}{3 \zeta}[W(X)-W(-\infty)]+O\left(\zeta^{-2}\right)
$$

i.e., $W(X)-W(-\infty)=\lim _{\zeta \rightarrow \infty}[3 \zeta(1-\psi \exp (-\zeta X))]$. Taking into account the functional dependence (56), we find the required one-soliton solution of the VPE in form

$$
W(X, T)=6 \sqrt{3} \xi_{1} \frac{\partial}{\partial X} \ln \left[1+\frac{\beta_{1}}{2 \sqrt{3}} \exp \left(\sqrt{3} \xi_{1} X-\frac{1}{\sqrt{3} \xi_{1}} T\right)\right]+\text { const. }
$$

Thus, for the example of the one-soliton solution, we have demonstrated the IST method.

\subsection{The direct spectral problem}

Let us consider the principal aspects of the inverse scattering transform problem for a third-order equation. The inverse problem for certain third-order spectral equations has been considered by Kaup [33] and Caudrey [34, 35]. The time evolution of $\psi$ is determined from (48) or (51).

Following the method described by Caudrey [34], the spectral equation (47) can be rewritten

$$
\frac{\partial}{\partial X} \psi=[\mathbf{A}(\zeta)+\mathbf{B}(X, \zeta)] \cdot \psi
$$

with

$$
\psi=\left(\begin{array}{l}
\psi \\
\psi_{X} \\
\psi_{X X}
\end{array}\right), \quad \mathbf{A}=\left(\begin{array}{ccc}
0 & 1 & 0 \\
0 & 0 & 1 \\
\lambda & 0 & 0
\end{array}\right), \quad \mathbf{B}=\left(\begin{array}{ccc}
0 & 0 & 0 \\
0 & 0 & 0 \\
0 & -W_{X} & 0
\end{array}\right) .
$$

The matrix A has eigenvalues $\lambda_{j}(\zeta)$ and left and right eigenvectors $\tilde{\boldsymbol{v}}_{j}(\zeta)$ and $\boldsymbol{v}_{j}(\zeta)$, respectively. These quantities are defined through a spectral parameter $\lambda$ as

$$
\begin{aligned}
& \lambda_{j}(\zeta)=\omega_{j} \zeta, \quad \lambda_{j}^{3}(\zeta)=\lambda, \\
& \boldsymbol{v}_{j}(\zeta)=\left(\begin{array}{c}
1 \\
\lambda_{j}(\zeta) \\
\lambda_{j}^{2}(\zeta)
\end{array}\right), \quad \tilde{\boldsymbol{v}}_{j}(\zeta)=\left(\begin{array}{lll}
\lambda_{j}^{2}(\zeta) & \lambda_{j}(\zeta) & 1
\end{array}\right),
\end{aligned}
$$


where, as previously, $\omega_{j}=e^{2 \pi \mathrm{i}(j-1) / 3}$ are the cube roots of $1(j=1,2,3)$. Obviously the $\lambda_{j}(\zeta)$ are distinct, and they and $\tilde{\boldsymbol{v}}_{j}(\zeta)$ and $\boldsymbol{v}_{j}(\zeta)$ are analytic throughout the complex $\zeta$-plane.

The solution of the linear equation (47) (or equivalently (59)) has been obtained by Caudrey [34] in terms of Jost functions $\phi_{j}(X, \zeta)$ which have the asymptotic behaviour:

$$
\boldsymbol{\Phi}_{j}(X, \zeta):=\exp \left\{-\lambda_{j}(\zeta) X\right\} \phi_{j}(X, \zeta) \rightarrow v_{j}(\zeta) \text { as } X \rightarrow-\infty .
$$

Caudrey [34] showed how Eq. (59) can be solved by expressing it as a Fredholm integral equation.

The complex $\zeta$-plane is to be divided into regions such that, in the interior of each region, the order of the numbers $\operatorname{Re}\left(\lambda_{i}(\zeta)\right)$ is fixed. As we pass from one region to another, this order changes, and hence, on a boundary between two regions, $\operatorname{Re}\left(\lambda_{i}(\zeta)\right)=\operatorname{Re}\left(\lambda_{j}(\zeta)\right)$ for at least one pair $i \neq j$. The Jost function $\phi_{j}$ is regular throughout the complex $\zeta$-plane apart from poles and finite singularities on the boundaries between the regions. At any point in the interior of any region of the complex $\zeta$-plane, the solution of Eq. (59) is obtained by the relation (2.12) from [34]. It is the direct spectral problem.

\subsection{The spectral data}

The information about the singularities of the Jost functions $\phi_{j}(X, \zeta)$ reside in the spectral data. First let us consider the poles. It is assumed that a pole $\zeta_{i}^{(k)}$ in $\phi_{i}(X, \zeta)$ is simple, does not coincide with a pole of $\phi_{j}(X, \zeta)$ and $j \neq i$ and does not lie on a boundary between two regions. Then, as proven in [34], the residue is

$$
\operatorname{Res} \phi_{i}\left(X, \zeta_{i}^{(k)}\right)=\sum_{\substack{j=1 \\ j \neq i}}^{n} \gamma_{i j}^{(k)} \phi_{j}\left(X, \zeta_{i}^{(k)}\right)
$$

and it can be found because we know the solution (47) in any regular regions from solving the direct problem (see Section 5.2). Note that, for $\phi_{j}\left(X, \zeta_{i}^{(k)}\right)$, the point $\zeta_{i}^{(k)}$ lies in the interior of a regular region. The quantities $\zeta_{i}^{(k)}$ and $\gamma_{i j}^{(k)}$ constitute the discrete part of the spectral data.

Now we consider the singularities on the boundaries between regions. However, in order to simplify matters, we first make some observations. The solution of the spectral problem can be facilitated by using various symmetry properties. In view of (47), we need only consider the first elements of

$$
\phi_{i}(X, \zeta)=\left(\begin{array}{c}
\phi_{i}(X, \zeta) \\
\phi_{i}(X, \zeta)_{X} \\
\phi_{i}(X, \zeta)_{X X}
\end{array}\right)
$$

whilst the symmetry

$$
\phi_{1}\left(X, \zeta / \omega_{1}\right)=\phi_{2}\left(X, \zeta / \omega_{2}\right)=\phi_{3}\left(X, \zeta / \omega_{3}\right)
$$

means we need only to consider $\phi_{1}(X, \zeta)$. In our case, for $\phi_{1}(X, \zeta)$, the complex $\zeta$-plane is divided into four regions by two lines (see Figure 5 ) given by 


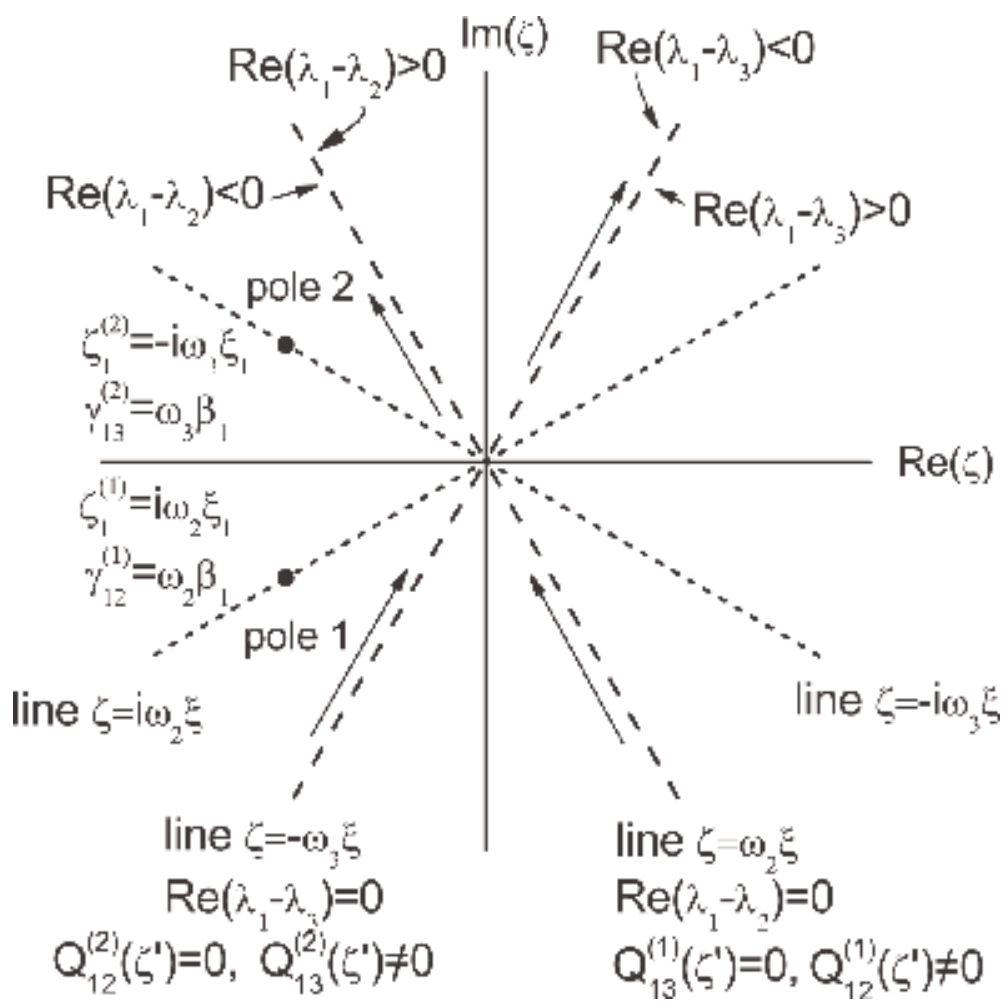

Figure 5.

The regular regions for Jost functions $\phi_{1}(X, \zeta)$ in the complex $\zeta$-plane. The dashed lines determine the boundaries between regular regions. These lines are lines where the singularity functions $Q_{i j}\left(\zeta^{\prime}\right)$ are given. The dotted lines are the lines where the poles appear.

$$
\begin{aligned}
& \text { (i) } \zeta^{\prime}=\omega_{2} \xi, \quad \text { where } \quad \operatorname{Re}\left(\lambda_{1}(\zeta)\right)=\operatorname{Re}\left(\lambda_{2}(\zeta)\right), \\
& \text { (ii) } \quad \zeta^{\prime}=-\omega_{3} \xi, \quad \text { where } \quad \operatorname{Re}\left(\lambda_{1}(\zeta)\right)=\operatorname{Re}\left(\lambda_{3}(\zeta)\right),
\end{aligned}
$$

where $\xi$ is real (see Figure 5). The singularity of $\phi_{1}(X, \zeta)$ can appear only on these boundaries between the regular regions on the $\zeta$-plane, and it is characterized by functions $Q_{1 j}\left(\zeta^{\prime}\right)$ at each fixed $j \neq 1$. We denote the limit of a quantity, as the boundary is approached, by the superfix \pm in according to the sign of $\operatorname{Re}\left(\lambda_{1}(\zeta)-\lambda_{j}(\zeta)\right)$ (see Figure 5).

In [34] (see Eq. (3.14) there) the jump of $\phi_{1}(X, \zeta)$ on the boundaries is calculated as

$$
\phi_{1}^{+}(X, \zeta)-\phi_{1}^{-}(X, \zeta)=\sum_{j=2}^{3} Q_{1 j}(\zeta) \phi_{j}^{-}(X, \zeta)
$$

where, from (66), the sum is over the lines $\zeta^{\prime}=\omega_{2} \xi$ and $\zeta^{\prime}=-\omega_{3} \xi$ given by

(i) $\quad \zeta^{\prime}=\omega_{2} \xi, \quad$ with $\quad Q_{12}^{(1)}\left(\zeta^{\prime}\right) \neq 0, \quad Q_{13}^{(1)}\left(\zeta^{\prime}\right) \equiv 0$,

(ii) $\quad \zeta^{\prime}=-\omega_{3} \xi, \quad$ with $\quad Q_{12}^{(2)}\left(\zeta^{\prime}\right) \equiv 0, \quad Q_{13}^{(2)}\left(\zeta^{\prime}\right) \neq 0$.

The singularity functions $Q_{1 j}\left(\zeta^{\prime}\right)$ are determined by $W(X, 0)$ through the matrix $\mathbf{B}(X, \zeta)(60)$ (see Eq. (3.13) in [34]) 


$$
Q_{1 j}(\zeta)=\frac{1}{\tilde{\boldsymbol{v}}_{j}(\zeta) \cdot v_{j}(\zeta)} \quad \tilde{\mathbf{v}}_{j}(\zeta) \cdot \int_{-\infty}^{\infty} \exp \left[\left(\lambda_{1}(\zeta)-\lambda_{j}(\zeta)\right) z\right] \mathbf{B}(z, \zeta) \cdot \phi_{1}^{-}(X, \zeta) d z
$$

The quantities $Q_{1 j}\left(\zeta^{\prime}\right)$ along all the boundaries constitute the continuum part of the spectral data.

Thus, the spectral data are

$$
S=\left\{\zeta_{1}^{(k)}, \gamma_{1 j}^{(k)}, Q_{1 j}\left(\zeta^{\prime}\right) ; j=2,3, k=1,2, \ldots, m\right\} .
$$

One of the important features which is to be noted for the IST method is as follows. After the spectral data have been found from $\mathbf{B}(X, 0 ; \zeta)$, i.e., at initial time, we need to seek the time evolution of the spectral data from Eq. (48). Analysing (48) at $X \rightarrow \infty$ together with (62)

$$
\phi_{i}(X, T, \zeta)=\exp \left[-\left(3 \lambda_{i}(\zeta)\right)^{-1} T\right] \phi_{i}(X, 0, \zeta),
$$

the $T$-dependence is revealed as

$$
\begin{aligned}
\zeta_{j}^{(k)}(T) & =\zeta_{j}^{(k)}(0), \\
\gamma_{1 j}^{(k)}(T) & =\gamma_{1 j}^{(k)}(0) \exp \left\{\left[-\left(3 \lambda_{j}\left(\zeta_{1}^{(k)}\right)\right)^{-1}+\left(3 \lambda_{1}\left(\zeta_{1}^{(k)}\right)\right)^{-1}\right] T\right\}, \\
Q_{1 j}\left(T ; \zeta^{\prime}\right) & =Q_{1 j}\left(0 ; \zeta^{\prime}\right) \exp \left\{\left[-\left(3 \lambda_{j}\left(\zeta^{\prime}\right)\right)^{-1}+\left(3 \lambda_{1}\left(\zeta^{\prime}\right)\right)^{-1}\right] T\right\} .
\end{aligned}
$$

The final step in the application of the IST method is to reconstruct $B(X, T ; \zeta)$ from the evaluated spectral data. In the next section, we show how to do this.

\subsection{The inverse spectral problem}

The final procedure in IST method is that of the reconstruction of the matrix $B(X, T ; \zeta)$ and $W(X, T)$ from the spectral data $S$.

The spectral data define $\Phi_{1}(X, \zeta)$ uniquely in the form (see Eq. (6.20) in [34]))

$$
\begin{aligned}
\Phi_{1}(X, T ; \zeta)= & 1-\sum_{k=1 j=2}^{K} \sum_{1 j}^{3} \gamma_{1 j}^{(k)}(T) \frac{\exp \left\{\left[\lambda_{j}\left(\zeta_{1}^{(k)}\right)-\lambda_{1}\left(\zeta_{1}^{(k)}\right)\right] X\right\}}{\lambda_{1}\left(\zeta_{1}^{(k)}\right)-\lambda_{1}(\zeta)} \Phi_{1}\left(X, T ; \omega_{j} \zeta_{1}^{(k)}\right) \\
& +\frac{1}{2 \pi \mathrm{i}} \int \sum_{j=2}^{3} Q_{1 j}\left(T ; \zeta^{\prime}\right) \frac{\exp \left\{\left[\lambda_{j}\left(\zeta^{\prime}\right)-\lambda_{1}\left(\zeta^{\prime}\right)\right] X\right\}}{\zeta^{\prime}-\zeta} \Phi_{1}^{-}\left(X, T ; \omega_{j} \zeta^{\prime}\right) d \zeta^{\prime} .
\end{aligned}
$$

Eq. (71) contains the spectral data, namely, $K$ poles with the quantities $\gamma_{1 j}^{(k)}$ for the bound state spectrum as well as the functions $Q_{1 j}\left(\zeta^{\prime}\right)$ given along all the boundaries of regular regions for the continuous spectrum. The integral in (71) is along all the boundaries (see the dashed lines in Figure 5). The direction of integration is taken so that the side chosen to be $\operatorname{Re}\left(\lambda_{1}(\zeta)-\lambda_{j}(\zeta)\right)<0$ is shown by the arrows in Figure 5 (for the lines (66), $\xi$ sweeps from $-\infty$ to $+\infty$ ). 
It is necessary to note that we should carry out the integration along the lines $\omega_{2}(\xi+\mathrm{i} \varepsilon)$ and $-\omega_{3}(\xi+\mathrm{i} \varepsilon)$ with $\varepsilon>0$. In this case condition (62) is satisfied. Passing to the limit $\varepsilon \rightarrow 0$, we can obtain the solution which does not satisfy condition (62). However, for any finite $\varepsilon>0$, the restricted region on $X$ can be determined where the solution associated with a finite $\varepsilon>0$ (for which the condition (62) is valid) and the solution associated with $\varepsilon=0$ are sufficiently close to each other. In this sense, taking the integration at $\varepsilon=0$, we remain within the inverse scattering theory [34], and so condition (62) can be omitted. The solution obtained at $\varepsilon=0$ can be extended to sufficiently large finite $X$. Thus, we will interpret the solution obtained at $\varepsilon=0$ as the solution of the VPE (27) which is valid for arbitrary but finite $X$.

By choosing appropriate values for $\zeta$, the left-hand side in (71) can be $\Phi_{1}\left(X, T ; \omega_{j} \zeta_{1}^{(k)}\right)$, or by allowing $\zeta$ to approach the boundaries from the appropriate sides, the left-hand side can be $\Phi_{1}^{-}\left(X, T ; \omega_{j} \zeta^{\prime}\right)$. We acquire a set of linear matrix/ Fredholm equations in the unknowns $\Phi_{1}\left(X, T ; \omega_{j} \zeta_{1}^{(k)}\right)$ and $\Phi_{1}^{-}\left(X, T ; \omega_{j} \zeta^{\prime}\right)$. The solution of this equation system enables one to define $\Phi_{1}(X, T ; \zeta)$ from (71).

By knowing $\Phi_{1}(X, T ; \zeta)$, we can take extra information into account, namely, that the expansion of $\Phi_{1}(X, T ; \zeta)$ as an asymptotic series in $\lambda_{1}^{-1}(\zeta)$ connects with $W(X, T)$ as follows (cf. Eq. (2.7) in [33]):

$$
\Phi_{1}(X, T ; \zeta)=1-\frac{1}{3 \lambda_{1}(\zeta)}[W(X, T)-W(-\infty)]+O\left(\lambda_{1}^{-2}(\zeta)\right)
$$

Consequently, the solution $W(X, T)$ and the matrix $B(X, T ; \zeta)$ can be reconstructed from the spectral data.

\section{The interaction of the loop-like solitons}

We will discuss the exact $N$-soliton solution of the VPE via the inverse scattering method [24]. To do this we consider (71) with $Q_{1 j}(\zeta) \equiv 0$. Then there is only the bound state spectrum which is associated with the soliton solutions.

Let the bound state spectrum be defined by $K$ poles. The relation (71) is reduced to the form

$$
\Phi_{1}(X, T ; \zeta)=1-\sum_{k=1}^{K} \sum_{j=2}^{3} \gamma_{1 j}^{(k)}(T) \frac{\exp \left\{\left[\lambda_{j}\left(\zeta_{1}^{(k)}\right)-\lambda_{1}\left(\zeta_{1}^{(k)}\right)\right] X\right\}}{\lambda_{1}\left(\zeta_{1}^{(k)}\right)-\lambda_{1}(\zeta)} \Phi_{1}\left(X, T ; \omega_{j} \zeta_{1}^{(k)}\right) .
$$

Eq. (73) involves the spectral data, namely, the poles $\zeta_{1}^{(k)}$ and the quantities $\gamma_{1 j}^{(k)}$. First we will prove that $\operatorname{Re} \lambda=0$ for compact support. From Eq. (47) we have

$$
\left(\psi_{X}\right)_{X X X}+\left(U \psi_{X}\right)_{X}-\lambda \psi_{X}=0,
$$

and together with Eq. (47), this enables us to write

$$
\frac{\partial}{\partial X}\left(\frac{\partial^{2}}{\partial X^{2}} \psi_{X} \psi^{*}-3 \psi_{X X} \psi_{X}^{*}+U \psi_{X} \psi^{*}\right)-2 \operatorname{Re} \lambda \psi_{X} \psi^{*}=0
$$

Integrating Eq. (75) over all values of $X$, we obtain that, for compact support, $\operatorname{Re} \lambda=0$ since, in the general case, $\int_{-\infty}^{\infty} \psi_{X} \psi^{*} d X \neq 0$. 
As follows from Eqs. (2.12), (2.13), (2.36) and (2.37) of [33], $\psi_{X}(\zeta)$ is related to the adjoint states $\psi_{X}^{A}(-\zeta)$. In the usual manner, using the adjoint states and Eq. (14) from [35] and Eq. (2.37) from [33], one can obtain

$$
\phi_{1 X}(X, \zeta)=\frac{\mathrm{i}}{\sqrt{3}}\left[\phi_{1 X}\left(X,-\omega_{2} \zeta\right) \phi_{1}\left(X,-\omega_{3} \zeta\right)-\phi_{1 X}\left(X,-\omega_{3} \zeta\right) \phi_{1}\left(X,-\omega_{2} \zeta\right)\right]
$$

It is easily seen that if $\zeta_{1}^{(1)}$ is a pole of $\phi_{1}(X, \zeta)$, then there is a pole either at $\zeta_{1}^{(2)}=-\omega_{2} \zeta_{1}^{(1)}$ (if $\phi_{1}\left(X,-\omega_{2} \zeta\right)$ has a pole) or at $\zeta_{1}^{(2)}=-\omega_{3} \zeta_{1}^{(1)}$ (if $\phi_{1}\left(X,-\omega_{3} \zeta\right)$ has a pole). For definiteness let $\zeta_{1}^{(2)}=-\omega_{2} \zeta_{1}^{(1)}$. Then, as follows from (76), $-\omega_{3} \zeta_{1}^{(2)}$ should be a pole. However, this pole coincides with pole $\zeta_{1}^{(1)}$, since $-\omega_{3} \zeta_{1}^{(2)}=$ $-\omega_{3}\left(-\omega_{2}\right) \zeta_{1}^{(1)}=\zeta_{1}^{(1)}$. Hence, the poles appear in pairs, $\zeta_{1}^{(2 n-1)}$ and $\zeta_{1}^{(2 n)}$, under the condition $\zeta_{1}^{(2 n)} / \zeta_{1}^{(2 n-1)}=-\omega_{2}$, where $n$ is the pair number.

Let us consider $N$ pairs of poles, i.e., in all there are $K=2 N$ poles over which the sum is taken in (76). For the pair $n(n=1,2, \ldots, N)$ we have the properties

$$
\text { (i) } \zeta_{1}^{(2 n-1)}=\mathrm{i} \omega_{2} \xi_{n}, \quad \text { (ii) } \zeta_{1}^{(2 n)}=-\mathrm{i} \omega_{3} \xi_{n} .
$$

Since $U$ is real and $\lambda$ is imaginary, $\xi_{k}$ is real. The relationships (77) are in line with the condition (2.33) from [33]. These relationships are also similar to Eqs. (6.24) and (6.25) in [34], whilst $\gamma_{1 j}^{(k)}$ turns out to be different from $\tilde{\gamma}_{1 j}^{(k)}$ for the Boussinesq equation (see Eqs. (6.24) and (6.25) in [34]). Indeed, by considering (76) in the vicinity of the first pole $\zeta_{1}^{(2 n-1)}$ of the pair $n$ and using the relation (73), one can obtain a relation between $\gamma_{12}^{(k)}$ and $\gamma_{13}^{(k)}$. In this case the functions $\phi_{1, X}(X, \zeta)$, $\phi_{1}\left(X,-\omega_{2} \zeta\right)$ and $\phi_{1, X}\left(X,-\omega_{2} \zeta\right)$ also have poles here, whilst the functions $\phi_{1}\left(X,-\omega_{3} \zeta\right)$ and $\phi_{1, X}\left(X,-\omega_{3} \zeta\right)$ do not have poles here. Substituting $\phi_{1}(X, \zeta)$ in the form (73) into Eq. (76) and letting $X \rightarrow-\infty$, we have the ratio $\gamma_{13}^{(2 n)} / \gamma_{12}^{(2 n-1)}=\omega_{2}$ and $\gamma_{12}^{(2 n)}=\gamma_{13}^{(2 n-1)}=0$. Therefore, the properties of $\gamma_{i j}^{(k)}$ should be defined by the relationships

$$
\begin{aligned}
& \text { (i) } \gamma_{12}^{(2 n-1)}=\omega_{2} \beta_{k}, \quad \gamma_{13}^{(2 n-1)}=0, \\
& \text { (ii) } \gamma_{12}^{(2 n)}=0, \quad \gamma_{13}^{(2 n)}=\omega_{3} \beta_{k},
\end{aligned}
$$

where, as it will be proved below, $\beta_{k}$ is real when $U=W_{X}$ is real.

By defining

$$
\Psi_{k}(X, T)=\sum_{j=2}^{3} \gamma_{1 j}^{(k)}(T) \exp \left\{\lambda_{j}\left(\zeta_{1}^{(k)}\right) X\right\} \Phi_{1}\left(X, T ; \omega_{j} \zeta_{1}^{(k)}\right),
$$

we may rewrite the relationship (73) as (see, for instance, Eqs. (6.33) and (6.34) in [34])

$$
\Phi_{1}(X, T ; \zeta)=1-\sum_{k=1}^{2 N} \frac{\exp \left\{-\lambda_{1}\left(\zeta_{1}^{(k)}\right) X\right\}}{\lambda_{1}\left(\zeta_{1}^{(k)}\right)-\lambda_{1}(\zeta)} \Psi_{k}(X, T) .
$$

From (72) and (80), it may be shown that (cf. Eq. (6.38) in [34])

$$
W(X, T)-W(-\infty)=-3 \sum_{k=1}^{2 N} \exp \left\{-\lambda_{1}\left(\zeta_{1}^{(k)}\right) X\right\} \Psi_{k}(X, T)=3 \frac{\partial}{\partial X} \ln (\operatorname{det} M(X, T)) .
$$


The $2 N \times 2 N$ matrix $M(X, T)$ is defined as in relationship (6.36) in [34] by

$M_{k l}(X, T)=\delta_{k l}-\sum_{j=2}^{3} \gamma_{1 j}^{(k)}(0) \frac{\exp \left\{\left[-\left(3 \lambda_{j}\left(\zeta_{1}^{(k)}\right)\right)^{-1}+\left(3 \lambda_{1}\left(\zeta_{1}^{(k)}\right)\right)^{-1}\right] T+\left(\lambda_{j}\left(\zeta_{1}^{(k)}\right)-\lambda_{1}\left(\zeta_{1}^{(l)}\right)\right) X\right\}}{\lambda_{j}\left(\zeta_{1}^{(k)}\right)-\lambda_{1}\left(\zeta_{1}^{(l)}\right)}$,

and

$$
\begin{aligned}
& n=1,2, \ldots, N \text {, } \\
& \lambda_{1}\left(\zeta_{1}^{(2 n-1)}\right)=i \omega_{2} \xi_{n}, \quad \lambda_{2}\left(\zeta_{1}^{(2 n-1)}\right)=i \omega_{3} \xi_{n}, \quad \gamma_{12}^{(2 n-1)}=\omega_{2} \beta_{n}, \quad \gamma_{13}^{(2 n-1)}=0, \\
& \lambda_{1}\left(\zeta_{1}^{(2 n)}\right)=-i \omega_{3} \xi_{n}, \quad \lambda_{3}\left(\zeta_{1}^{(2 n)}\right)=-i \omega_{2} \xi_{n}, \quad \gamma_{12}^{(2 n)}=0, \quad \gamma_{13}^{(2 n)}=\omega_{3} \beta_{n} .
\end{aligned}
$$

For the $N$-soliton solution, there are $N$ arbitrary constants $\xi_{n}$ and $N$ arbitrary constants $\beta_{n}$.

The final result for the $N$-soliton solution of the VPE is defined by relationship (81) with (82).

\subsection{Examples of one- and two-soliton solutions of the VPE}

In order to obtain the one-soliton solution of the VPE (27)

$$
W_{X X T}+\left(1+W_{T}\right) W_{X}=0,
$$

we need first to calculate the $2 \times 2$ matrix $M(X, T)$ according to (82) with $N=1$. We find that the matrix is

$$
\left(\begin{array}{ll}
1-\frac{\omega_{2} \beta_{1}}{\sqrt{3} \xi_{1}} \exp \left[\sqrt{3} \xi_{1} X-\left(\sqrt{3} \xi_{1}\right)^{-1} T\right] & \frac{\mathrm{i} \omega_{3} \beta_{1}}{2 \xi_{1}} \exp \left[2 \mathrm{i} \omega_{3} \xi_{1} X-\left(\sqrt{3} \xi_{1}\right)^{-1} T\right] \\
\frac{-\mathrm{i} \omega_{2} \beta_{1}}{2 \xi_{1}} \exp \left[-2 \mathrm{i} \omega_{2} \xi_{1} X-\left(\sqrt{3} \xi_{1}\right)^{-1} T\right] & 1-\frac{\omega_{3} \beta_{1}}{\sqrt{3} \xi_{1}} \exp \left[\sqrt{3} \xi_{1} X-\left(\sqrt{3} \xi_{1}\right)^{-1} T\right]
\end{array}\right)
$$

and its determinant is

$$
\operatorname{det} M(X, T)=\left\{1+\frac{\beta_{1}}{2 \sqrt{3} \xi_{1}} \exp \left[\sqrt{3} \xi_{1}\left(X-\frac{T}{3 \xi_{1}^{2}}\right)\right]\right\}^{2}
$$

Consequently, from Eq. (81) we have the one-soliton solution of the VPE

$$
U(X, T)=W_{X}(X, T)=\frac{9}{2} \xi_{1}^{2} \operatorname{sech}^{2}\left[\frac{\sqrt{3}}{2} \xi_{1}\left(X-\frac{T}{3 \xi_{1}^{2}}\right)+\alpha_{1}\right],
$$

where $\alpha_{1}=\frac{1}{2} \ln \left(\beta_{1} / 2 \sqrt{3} \xi_{1}\right)$ is an arbitrary constant. Since $U$ is real, it follows from (85) that $\beta_{1}$ is real. Note that with $\beta_{1} / \xi_{1}<0$ we have the real solution in the form of the singular soliton [41]. 


$$
U(X, T)=\frac{9}{2} \xi_{1}^{2} \sinh ^{-2} \eta, \quad \eta=\frac{\sqrt{3}}{2} \xi_{1}\left(X-\frac{T}{3 \xi_{1}^{2}}\right)+\alpha_{1}
$$

Let us now consider the two-soliton solution of the VPE. In this case $M(X, T)$ is a $4 \times 4$ matrix. We will not give the explicit form here, but we find that

$$
\operatorname{det} M(X, T)=\left(1+q_{1}^{2}+q_{2}^{2}+b^{2} q_{1}^{2} q_{2}^{2}\right)^{2},
$$

where

$$
q_{i}=\exp \left[\frac{\sqrt{3}}{2} \xi_{i}\left(X-\frac{T}{3 \xi_{i}^{2}}\right)+\alpha_{i}\right], \quad b^{2}=\left(\frac{\xi_{2}-\xi_{1}}{\xi_{2}+\xi_{1}}\right)^{2} \frac{\xi_{1}^{2}+\xi_{2}^{2}-\xi_{1} \xi_{2}}{\xi_{1}^{2}+\xi_{2}^{2}+\xi_{1} \xi_{2}},
$$

and $\alpha_{i}=\frac{1}{2} \ln \left(\beta_{i} / 2 \sqrt{3} \xi_{i}\right)$ are arbitrary constants. The two-soliton solution to the VPE as found by the IST method is given by (81) together with (87).

Finally we note that comparison of (81) with $W=6(\ln f)_{X}$ from (42) shows that

$$
\ln (\operatorname{det} M(X, T))=2 \ln (f) .
$$

so that $\operatorname{det} M(X, T)$ is a perfect square for arbitrary $N$.

\subsection{The two-loop-like solitons of the VE}

We discuss the two-loop soliton solution of the VE in more detail. Let us consider what happens in $x$ - $t$ space. The relations (20), (25) and (29) determine the solutions in $x-t$ throughout the solutions in $X$-T. In these coordinates $x$ - $t$, we have the loop-like solitons.

The shifts, $\delta_{i}$, of the two-loop solitons $u_{1}$ and $u_{2}$ in the positive $x$-direction due to the interaction may be computed as follows. The larger loop soliton is always shifted forwards by the interaction. However, for smaller $u_{2}$ with $r=\xi_{1} / \xi_{2}$, there is a value $r_{c}=0.88867$ in that we have a different form of the phase shift:

a. For $r_{c}<r<1, \delta_{1}<0$ so the smaller loop soliton is shifted backwards.

b. For $r=r_{c}$, where $r_{c}=0.88867$ is the root of $\ln b+3 / r=0, \delta_{1}=0$, so the smaller loop soliton is not shifted by the interaction.

c. For $0<r<r_{c}, \delta_{1}>0$ so the smaller loop soliton is shifted forwards.

At first sight it might seem that the behaviour in (b) and (c) contradicts conservation of 'momentum'. That this is not so is justified as follows. By integrating (9) with respect to $x$, we find that $\int_{-\infty}^{\infty} u d x=0$; also, by multiplying (9) by $x$ and integrating with respect to $x$, we obtain $\int_{-\infty}^{\infty} x u d x=0$. Thus, in $x-t$ space, the 'mass' of each soliton is zero, and 'momentum' is conserved whatever $\delta_{1}$ and $\delta_{2}$ may be. In particular $\delta_{1}$ and $\delta_{2}$ may have the same sign as in (c), or one of them may be zero as in (b).

Cases (a), (b) and (c) are illustrated in Figures 6-8, respectively; in these figures $u$ is plotted against $x$ for various values of $t$. For convenience in the figures, the interactions of solitons are shown in coordinates moving with speed $\left(v_{1}+v_{2}\right) / 2$. 


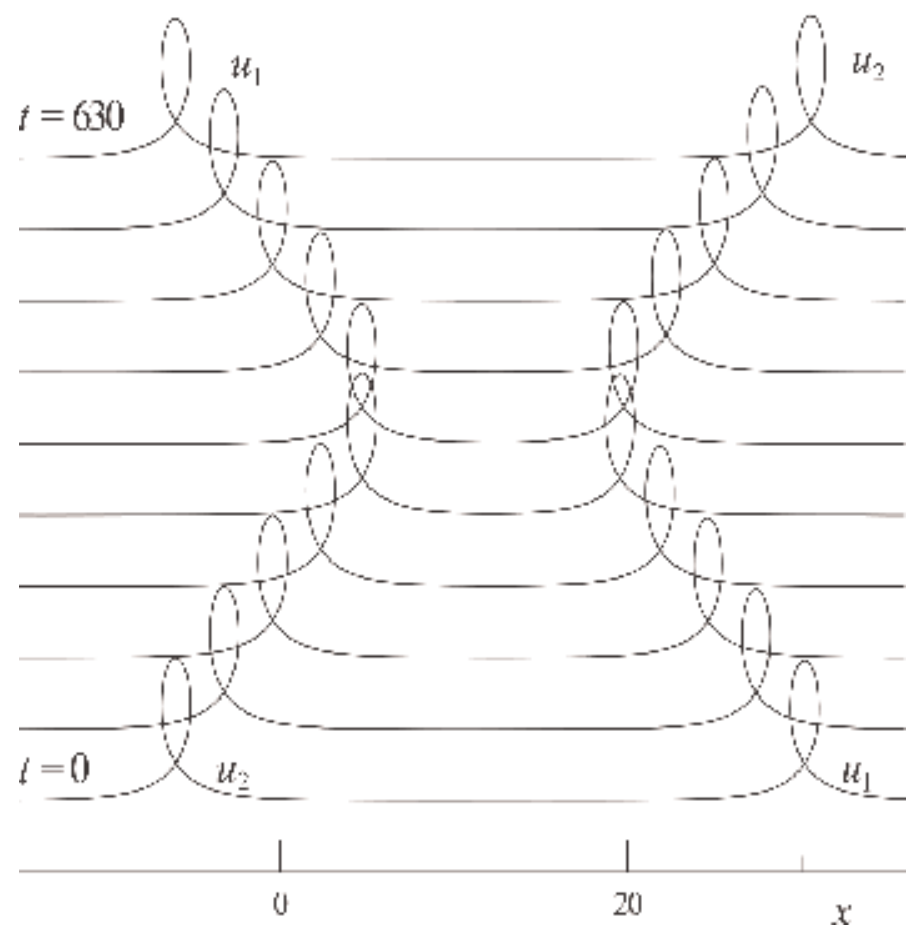

Figure 6.

The interaction process for two-loop solitons with $\xi_{1}=0.99$ and $\xi_{2}=1$ so that $r=0.99$ and $\delta_{1}<0$.

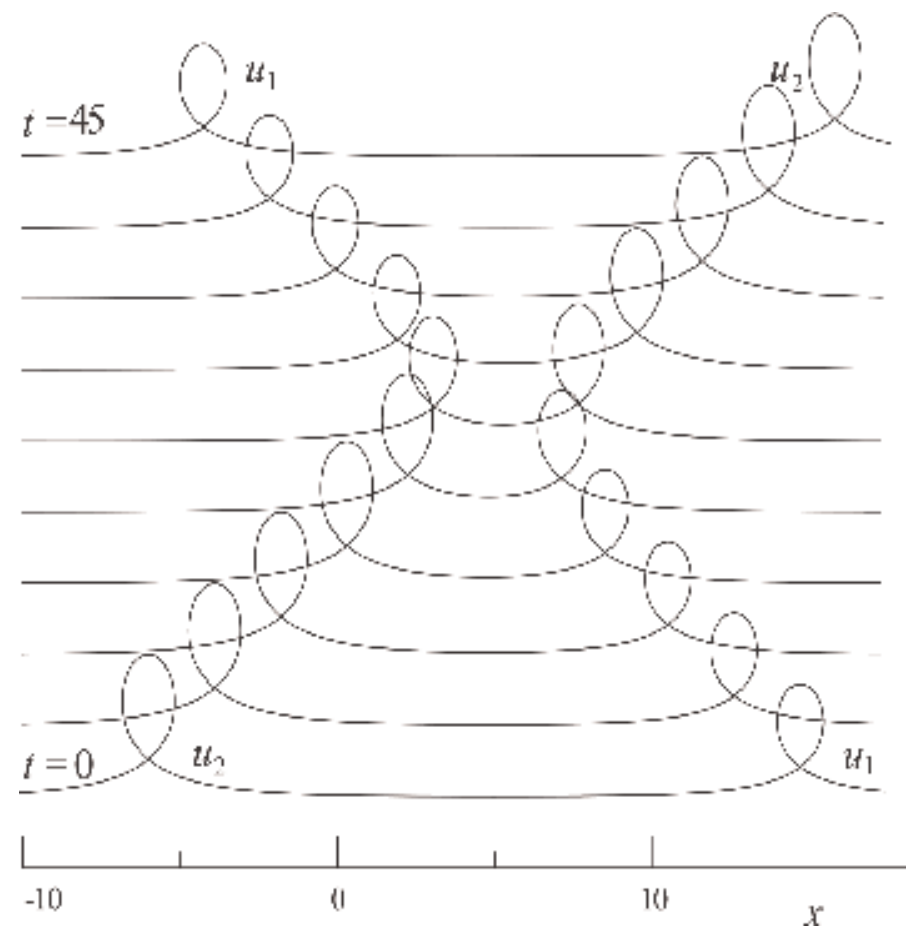

Figure 7.

The interaction process for two-loop solitons with $\xi_{1}=0.88867$ and $\xi_{2}=1$ so that $r=0.88867$ and $\delta_{1}=0$. 


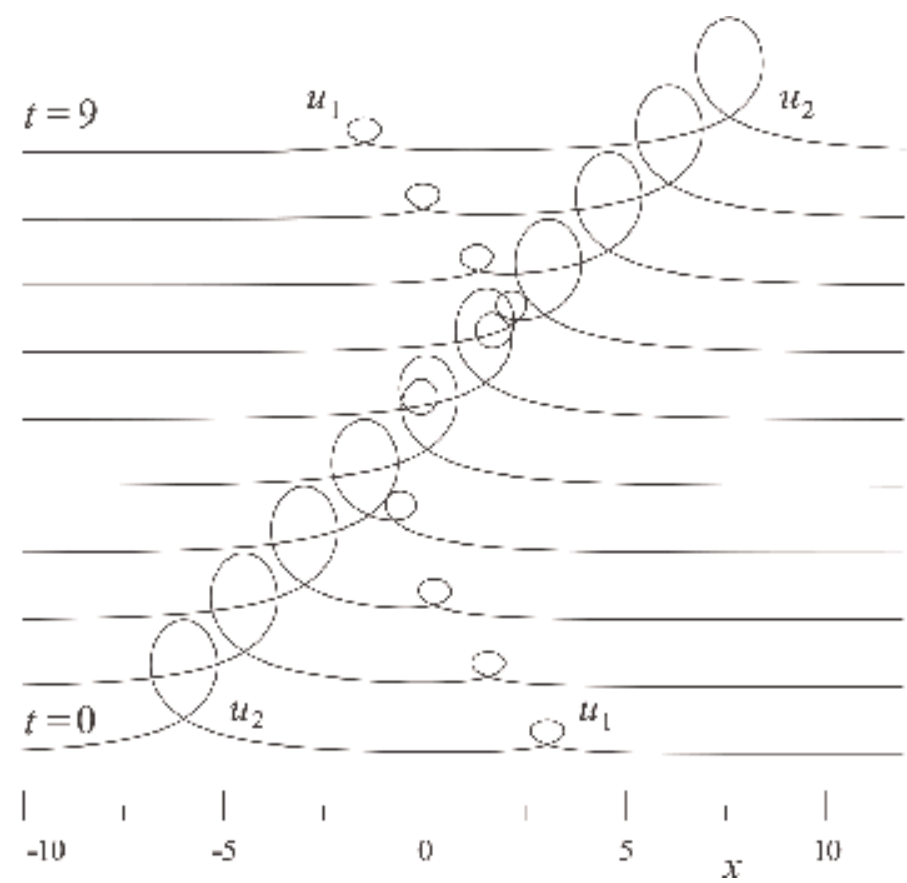

Figure 8.

The interaction process for two-loop solitons with $\xi_{1}=0.5$ and $\xi_{2}=1$ so that $r=0.5$ and $\delta_{1}>0$.

\section{Discussion on the loop-like solutions}

We have already mentioned the important question on stability of loop-like solutions (Section 2).

\subsection{Remarks on the existence and uniqueness theorem}

In [42], the existence and uniqueness theorem is formulated for system (one) differential equations. The loop-like solutions take place on travelling waves. In this case, the initial equation is reduced to an ordinary differential equation (ODE) (see Section 2). It has been this equation which we are exploring. Now we note some important remarks. In particular, in order to investigate the ODE (the solution on travelling waves), it is still necessary to reconcile this solution with the initial problem, which is described by the differential equation in partial derivatives (evolution equation). Consequently, the ambiguous solutions for the ODE during their reconstruction into the initial coordinates should be checked by means of some restrictive conditions (see 7.2 ).

It is necessary to note that if the conditions of the existence and uniqueness theorem break down, then nevertheless, this does not restrict the existence of solutions. Hence, the solutions can exist, for example, the multivalued solutions. Here we point out an example: the exact solutions for the Camassa-Holm equation (CHE) and the Degasperis-Procesi equation (DPE) can be constructed as the component solutions, through separate parts (branches) of solutions (see [43]).

The selection of possible multivalued solutions will be discussed in 7.2. 


\subsection{Selection for the loop-like solutions}

Solutions must satisfy the following conditions:

1. At the point $\eta=0$, the solution must pass over the ellipse $\left(z^{2}+2 v \eta^{2}\right)_{\eta \eta}=0$ (see Eq. (4.3) in [3]);

2. According to the conservation law $\int_{\infty}^{-\infty} u(x, t) d x=$ const $\equiv 0$ for $t>0$. The 'mass' of individual soliton equals to zero. This condition will be satisfied if point 1 takes place.

3. As you know [3], taking into account dissipation in the physical process allows one to select a solution from an array of possible solutions that are inherent to the equation without dissipation. This condition also selects a solution as in a point 1 if $\alpha \rightarrow 0$.

4.During the interaction of the solitons [24, 29], you must take into account all the parts of loop-like soliton (see end of Section 7.3). The soliton has a form satisfying point 2 .

Thus, we cannot arbitrarily combine the solutions at $\eta=0$. The solutions, in particular, solitons should be specific loop-like form.

\subsection{Physical interpretation of the multivalued solutions}

From the mathematical point of view, an ambiguous solution does not present difficulties, whereas the physical interpretation of ambiguity always presents some difficulties. In this connection the problem of ambiguous solutions is regarded as important. The problem consists in whether the ambiguity has a physical nature or is related to the incompleteness of the mathematical model, in particular to the lack of dissipation.

We will consider the problem related to the singular points when dissipation takes place. At these points the dissipative term $\alpha \frac{\partial u}{\partial x}$ tends to infinity. The question arises: Are there solutions of Eq. (8) in a loop-like form? That the dissipation is likely to destroy the loop-like solutions can be associated with the following wellknown fact [5]. For the simplest nonlinear equation without dispersion and without dissipation, namely,

$$
\frac{\partial u}{\partial t}+u \frac{\partial u}{\partial x}=0
$$

any initial smooth solution with boundary conditions

$$
\left.u\right|_{x \rightarrow+\infty}=0,\left.\quad u\right|_{x \rightarrow-\infty}=u_{0}=\text { const. }>0
$$

becomes ambiguous in the final analysis. When dissipation is considered, we have the Burgers equation [47]:

$$
\frac{\partial u}{\partial t}+u \frac{\partial u}{\partial x}+\mu \frac{\partial^{2} u}{\partial x^{2}}=0
$$

The dissipative term in this equation and in Eq. (6) for low frequency is coincident. The inclusion of the dissipative term transforms the solutions so that they cannot be ambiguous as a result of evolution. The wave parameters are always 
unambiguous. What happens in our case for high frequency when the dissipative term has the form $\alpha u$ (see Eq. (18) in [29])? Will the inclusion of dissipation give rise to unambiguous solutions?

By direct integration of Eq. (8) (written in terms of the variables (11)) within the neighbourhood of singular points $z=0$ where $z_{\eta} \rightarrow \pm \infty$ and $z_{\tau} \ll z_{\eta}$, it can be derived (see [3]) that the dissipative term, with dissipation parameter less than some limit value $\alpha^{*}$, does not destroy the loop-like solutions. Now we give a physical interpretation to ambiguous solutions.

Since the solution to the VE has a parametric form (15) and (16), there is a space of variables in which the solution is a single-valued function. Hence, we can solve the problem of the ambiguous solution. A number of states with their thermodynamic parameters can occupy one microvolume. It is assumed that the interaction between the separated states occupying one microvolume can be neglected in comparison with the interaction between the particles of one thermodynamic state. Even if we take into account the interaction between the separated states in accordance with the dynamic state equation (5), for high frequencies, a dissipative term arises which is similar to the corresponding term in Eq. (7) but with the other relaxation time. In this sense the separated terms are distributed in space, but describing the wave process, we consider them as interpenetratable. A similar situation, when several components with different hydrodynamic parameters occupy one microvolume, has been assumed in mixture theory (see, for instance, [48]). Such a fundamental assumption in the theory of mixtures is physically impossible (see [48], p.7), but it is appropriate in the sense that separated components are multi-velocity interpenetratable continua.

Consequently, the following three observations show that, in the framework of the approach considered here, there are multivalued solutions when we model high-frequency wave processes: (1) All parts of loop-like solution are stable to perturbations. (2) Dissipation does not destroy the loop-like solutions. (3) The investigation regarding the interaction of the solitons has shown that it is necessary to take into account the whole ambiguous solution and not just the separate parts.

\subsection{Conclusion}

Loop-like solitons are a class of interesting wave phenomena, which take place in some nonlinear systems. This interest consisted not only in the interpretation of the solutions obtained but also in the explanation of the experimental results. The ambiguous structure of the loop-like solutions is similar to the loop soliton solution to an equation that models a stretched rope [44]. Loop-like solitons on a vortex filament were investigated by Hasimoto [45] and Lamb, Jr. [46]. The loop-like solutions appear in description of physical phenomena, in particular, electromagnetic terahertz pulses in asymmetric molecules [49], high-frequency perturbations in a relaxation medium $[3,50,51]$ and soliton in ferrites [52]. As a typical multivalued structure, loop soliton has been discussed in some possible physical fields including particle physics [53] and quantum field theory [54].

It must be admitted that we are a long way still from complete awareness of physical processes which can be described by loop-like solutions. However, the approach, considered here, will hopefully be interesting and useful in understanding the birth and death process for particles, since the mass and momentum of individual loop-like soliton are zero. Furthermore, the investigations in optics, magnetism and hydrodynamics clearly indicate the acceptability of the approach on loop-like solitons. Indeed, the phase shifts observed at interaction of solitons can be explained by means of loop-like solutions. 


\section{Author details}

Vyacheslav O. Vakhnenko ${ }^{1 *}$, E. John Parkes ${ }^{2}$ and Dmitri B. Vengrovich ${ }^{1}$

1 Institute of Geophysics, Ukrainian Academy of Sciences, Kyiv, Ukraine

2 Department of Mathematics, University of Strathclyde, Glasgow, UK

*Address all correspondence to: vakhnenko@ukr.net

\section{IntechOpen}

(C) 2019 The Author(s). Licensee IntechOpen. This chapter is distributed under the terms of the Creative Commons Attribution License (http://creativecommons.org/licenses/ by/3.0), which permits unrestricted use, distribution, and reproduction in any medium, provided the original work is properly cited. (cc) BY 


\section{References}

[1] Clarke J. Lectures on plane waves in reacting gases. Annales de Physique. 1984;9:211-306. DOI: 10.1051/anphys: 0198400902021100

[2] Landau L, Lifshitz E. Fluids Mechanics. New York: Pergamon Press; 1978. p. 536. DOI: $10.1002 /$ qj. 49710444026

[3] Vakhnenko V. High-frequency soliton-like waves in a relaxing medium. Journal of Mathematical Physics. 1999; 40:2011-2020. DOI: 10.1063/1.532847

[4] Gardner C, Greene J, Kruskal M, Miura R. Method for solving the Korteweg-deVries equation. Physical Review Letters. 1967;19:1095-1097. DOI: 10.1103/PhysRevLett.19.1095

[5] Dodd R, Eilbeck J, Gibbon J, Morris $\mathrm{H}$. Solitons and Nonlinear Wave Equations. London: Academic Press; 1982. p. 640 . DOI: $10.1002 /$ zamm.19850650811

[6] Su C, Gardner C. Korteweg-de Vries equation and generalizations. III. Derivation of Korteweg-de Vries equation and Burgers equation. Journal of Mathematical Physics. 1969;10: 536-539. DOI: 10.1063/1.1664873

[7] Ablowitz M, Segur H. Solitons and Inverse Scattering Transform. Philadelphia: SIAM Press; 1981. 426 p. DOI: $10.1137 / 1.9781611970883$

[8] Novikov S, Manakov S, Pitaevskii L, Zakharov V. Theory of Solitons. The Inverse Scattering Methods. New York —London: Plenum Publishing Corporation; 1984. p. 286. ISBN 978-0306-10977-5

[9] Hirota R. Direct methods in soliton theory. In: Bullough R, Caudrey P, editors. Solitons. New York-Berlin: Springer; 1980. pp. 157-176. DOI: 10.1007/978-3-642-81448-8
[10] Hirota R. The Direct Method in Soliton Theory. Cambridge: Cambridge University Press; 2004. 198 p. 10.1017/ CBO9780511543043

[11] Newell A. Solitons in Mathematics and Physics. Philadelphia: SIAM; 1985. p. 244. ISBN 0898711967

[12] Faddeev L, Takhtajan L.

Hamiltonian Methods in the Theory of Solitons. New York, Verlag: BerlinHeidelberg, Springer; 1987. p. 592. DOI: 10.1007/978-3-540-69969-9

[13] Wazwaz A. Partial Differential Equations and Solitary Waves Theory. Berlin-Heidelberg: Springer; 2009. p. 700. DOI: $10.1007 / 978-3-642-00251-9$

[14] Vakhnenko V. Solitons in a nonlinear model medium. Journal of Physics A: Mathematical and General. 1992;25:4181-4187. DOI: $10.1088 /$ 0305-4470/25/15/025

[15] Parkes J. The stability of solutions of Vakhnenko's equation. Journal of Physics A: Mathematical and General. 1993;26:6469-6475. DOI: $10.1088 /$ 0305-4470/26/22/040

[16] Ostrovsky L. Nonlinear internal waves in a rotating ocean. Oceanology. 1978;18:119-125. https://ci.nii.ac.jp/naid/ 10010464167 ID. (NAID) 10010464167

[17] Byrd P, Friedman D. Handbook of Elliptic Integrals for Engineers and Scientists. 2nd ed. Berlin: SpringerVerlag; 1971. 276 p. DOI: 10.1007/978-3642-65138-0

[18] Vakhnenko V, Parkes J. The two loop soliton solution of the Vakhnenko equation. Nonlinearity. 1998;11:14571464. DOI: 10.1088/0951-7715/11/6/001

[19] Morrison A, Parkes J, Vakhnenko V. The $N$ loop soliton solution of the 
Vakhnenko equation. Nonlinearity. 1999;12:1427-1437. DOI: 10.1088/ 0951-7715/12/5/314

[20] Estévez P. Reciprocal transformations for a spectral problem in 2+1 dimensions. Theoretical and Mathematical Physics. 2009;159: 763-769. DOI: $10.4213 /$ tmf6360

[21] Abazari R. Application of $\left(G^{\prime} / G\right)$ expansion method to travelling wave solutions of three nonlinear evolution equation. Computers and Fluids. 2010; 39:1957-1963. DOI: 10.1016/j. compfluid.2010.06.024

[22] Majida F, Trikib H, Hayatc T, Aldossaryd O, Biswase A. Solitary wave solutions of the Vakhnenko-Parkes equation. Nonlinear Analysis: Modelling and Control. 2012;17:60-66 https:// www.mii.lt/na/issues/NA_1701/ NA17105.pd

[23] Ye Y, Song J, Shen S, Di Y. New coherent structures of the VakhnenkoParkes equation. Results in Physics. 2012;2:170-174. DOI: 10.1016/j. rinp.2012.09.011

[24] Vakhnenko V, Parkes J. The calculation of multi-soliton solutions of the Vakhnenko equation by the inverse scattering method. Chaos, Solitons and Fractals. 2002;13:1819-1826. DOI: 10.1016/S0960-0779(01)00200-4

[25] Vakhnenko V, Parkes J. The singular solutions of a nonlinear evolution equation taking continuous part of the spectral data into account in inverse scattering method. Chaos, Solitons and Fractals. 2012;45:846-852. DOI: 10.1016/ j.chaos.2012.02.019

[26] Vakhnenko V, Parkes J. Solutions associated with discrete and continuous spectrums in the inverse scattering method for the Vakhnenko-Parkes equation. Progress in Theoretical Physics. 2012;127:593-613. DOI: 10.1143/ PTP.127.593
[27] Vakhnenko V, Parkes J. Special singularity function for continuous part of the spectral data in the associated eigenvalue problem for nonlinear equations. Journal of Mathematical Physics. 2012;53:063504. DOI: 10.1063/ 1.4726168

[28] Vakhnenko V, Parkes J. The inverse problem for some special spectral data. Chaos, Solitons and Fractals. 2016;82:

116-124. DOI: 10.1016/j. chaos.2015.11.012

[29] Vakhnenko V, Parkes J. Approach in theory of nonlinear evolution equations: The Vakhnenko-Parkes equation. Advances in Mathematical Physics. 2016;2016:1-39. DOI: 10.1155/2016/ 2916582. Article ID 2916582

[30] Vakhnenko V, Parkes J, Morrison A. A Bäcklund transformation and the inverse scattering transform method for the generalised Vakhnenko equation. Chaos, Solitons and Fractals. 2003;17: 683-692. DOI: 10.1016/S0960-0779(02) 00483-6

[31] Hirota R. A new form of Bäcklund transformations and its relation to the inverse scattering problem. Progress in Theoretical Physics. 1974;52:1498-1512. DOI: 10.1143/PTP.52.1498

[32] Satsuma J, Kaup D. A Bäcklund transformation for a higher order Korteweg-de Vries equation. Journal of the Physical Society of Japan. 1977;43: 692-697. DOI: 10.1143/JPSJ.43.692

[33] Kaup D. On the inverse scattering problem for cubic eigenvalue problems of the class $\psi_{x x x}+6 Q \psi_{x}+6 R \psi=\lambda \psi$. Studies in Applied Mathematics. 1980; 62:189-216. DOI: 10.1002/ sapm1980623189

[34] Caudrey P. The inverse problem for a general $N \times N$ spectral equation. Physica D. 1982;6:51-66. DOI: 10.1016/ 0167-2789(82)90004-5 
[35] Caudrey P. The inverse problem for the third order equation $u_{x x x}+q(x) u_{x}+r(x) u=-i \zeta^{3} u$. Physics Letters A. 1980;79:264-268. DOI: 10.1016/0375-9601(80)90343-6

[36] Zakharov V. On stochastization of one-dimensional chains of nonlinear oscillators. Soviet Physics - JETP. 1974;38: 108-110 http://cds.cern.ch/record/407338

[37] Deift P, Tomei C, Trubowitz E. Inverse scattering and the Boussinesq equation. Commun. Pure and Applied Mathematics Journal. 1982;35:567-628. DOI: $10.1002 /$ cpa. 3160350502

[38] Hirota R, Satsuma J. A variety of nonlinear network equations generated from the Bäcklund transformation for the Toda lattice. Progress of Theoretical Physics Supplement. 1976;59:64-100. DOI: 10.1143/PTPS.59.64

[39] Musette M, Conte R. Algorithmic method for deriving lax pairs from the invariant Painlevé analysis of nonlinear partial differential equations. Journal of Mathematical Physics. 1991;32:

1450-1457. DOI: 10.1063/1.529302

[40] Clarkson P, Mansfield E. Symmetry reductions and exact solutions of shallow water wave equations. Acta Applicandae Mathematicae. 1995;39: 245-276. DOI: 10.1007/BF00994636

[41] Wazwaz A. N-soliton solutions for the Vakhnenko equation and its generalized forms. Physica Scripta. 2010;82:065006(7). DOI: 10.1088/ 0031-8949/82/06/065006

[42] Pontryagin L. Ordinary Differential Equations. London: Addison-Wesley Publishing Company; 1962. p. 298. DOI: 10.1002/zamm.19630430924

[43] Lenells J. Traveling wave solutions of the Camassa-holm equation. Journal of differential equation. 2005;217:393-430. DOI: 10.1016/j.jde.2004.09.007
[44] Konno K, Ichikawa Y, Wadati M. A loop soliton propagating along a stretched rope. Journal of the Physical Society of Japan. 1981;50:1025-1026. DOI: 10.1143/JPSJ.50.1025

[45] Hasimoto H. A soliton on a vortex filament. Journal of Fluid Mechanics. 1972;51:477-485. DOI: $10.1017 /$ S0022112072002307

[46] Lamb G Jr. Solitons on moving space curves. Journal of Mathematical Physics. 1977;18:1654-1661. DOI: 10.1063/1.523453

[47] Burgers J. A mathematical model illustrating the theory of turbulence. In: von Mises R, von Kármán T, editors. Advances in Applied Mechanics. New York: Academic Press Inc.; 1948. pp. 171-199. DOI: 10.1016/S0065-2156 (08)70100-5

[48] Rajagopal K, Tao L. Mechanics of Mixtures. Singapore: World Scientific Publishing; 1995. p. 195. ISBN 9810215851

[49] Sazonov S, Ustinov N. Nonlinear propagation of the vector too short pulses in the medium with symmetric and asymmetric molecules. Journal of Experimental and Theoretical Physics. 2017;124:249-269. DOI: 10.7868/ S0044451017020043

[50] Kraenkel R, Leblond H, Manna M. An integrable evolution equation for surface waves in deep water. Journal of Physics A: Mathematical and Theoretical. 2014;47:025208(17). DOI: 10.1088/1751-8113/47/2/025208

[51] Kuetche V. Barothropic relaxing media under pressure perturbations: Nonlinear dynamics. Dynamics of Atmospheres and Oceans. 2015;72:21-37. DOI: 10.1016/j.dynatmoce.2015.10.001

[52] Kuetche V. Inhomogeneous exchange within ferrites: Magnetic solitons and their interactions. Journal 
of Magnetism and Magnetic Materials. 2016;398:70-81. DOI: 10.1016/j. jmmm.2015.08.120

[53] Schleif M, Wunsch R.

Thermodynamic properties of the SU(2) (f) chiral quark-loop soliton. European Physical Journal A: Hadrons and Nuclei. 1998;1:171-186. DOI: 10.1007/ s100500050046

[54] Matsutani S. The relation of lemniscate and a loop soliton as 3/2 and 1 spin fields along the modified Korteweg-de Vries equation. Modern Physics Letters A. 1995;10:717-721. DOI: 10.1142/S0217732395000764 


\title{
Complex Dynamical Behavior of a Bounded Rational Duopoly Game with Consumer Surplus
}

\author{
Wei Zhou and Tong Chu
}

\begin{abstract}
In this chapter, we assume that two bounded rational firms not only pursue profit maximization but also take consumer surplus into account, so the objections of all the firms are combination of their profits and the consumer surplus. And then a dynamical duopoly Cournot model with bounded rationality is established. The existence and stability of the boundary equilibrium points and the Nash equilibrium of the model are discussed, respectively. And then the stability condition of the Nash equilibrium is given. The complex dynamical behavior of the system varies with parameters in the parameter space is studied by using the so-called $2 \mathrm{D}$ bifurcation diagram. The coexistence of multiple attractors is discussed through analyzing basins of attraction. It is found that not only two attractors can coexist, but also three or even four attractors may coexist in the established model. Then, the topological structure of basins of attraction and the global dynamics of the system are discussed through invertible map, critical curve and transverse Lyapunov exponent. At last, the synchronization phenomenon of the built model is studied.
\end{abstract}

Keywords: bifurcation, chaos, duopoly, consumer surplus, synchronization

\section{Introduction}

Oligopoly is a market between perfect monopoly and perfect competition [1]. With the application of chaos theory and nonlinear dynamic system into oligopoly models, the static game evolves into a dynamic game. Especially in recent years, with the rapid development of computer technology, a powerful tool has been provided for dealing with the complex nonlinear problems. And hence, the economists and the mathematicians can simulate the complex dynamical behavior of oligopoly market by using computer technology. Recently, a large number of scholars have improved the oligopoly models, and introduced bounded rationality (see $[2,3])$, incomplete information [4], time delay [5], market entering and entering barriers [6], differentiated products [7] and other factors [8,9] into the classical oligopoly models, and the bifurcation and chaos phenomenon were founded in the process of repeated game.

However, all of the above discussions are mainly based on private enterprises, which pursuit the maximization of their own profits. In fact, the public ownership enterprises, which always aim at maximizing social welfare, and mixed ownership enterprises, which always aim at maximizing the weighted average of the social 
welfare and their own profits, are also widespread in the real economic environment. De Fraja and Delbono [10] found that the social welfare might be higher when a public ownership enterprise is a profit-maximizer rather than a social-welfaremaximizer. Matsumura [11] proposed that the social welfare could be improved through partial privatization of public enterprises. The research of Fujiwara [12] suggested that partial privatized public enterprises are more efficient than private enterprises. Elsadany and Awad [13] explored the complex dynamical behavior of competition between two partial public enterprises under the assumption of bounded rationality. However, the global dynamical behavior and synchronization behavior of semi-public enterprises, which corporate social responsibility into their objectives, are rarely studied. In this chapter, the occurrence of synchronization, the coexistence of attractors and the global dynamic of a duopoly game corporate consumer surplus are mainly discussed.

\section{The model}

Considering a duopolistic market where two firms produce homogeneous goods. In order to study the long-term behaviors of the duopoly market with quantity competition, we briefly present the economic setup leading to the final model in this chapter. The price and quantity of product of firm $i$ are given by $p_{i}$ and $q_{i}$ respectively, with $i=1,2$. We also assume the existence of a continuum of identical consumers which have preferences toward $q_{1}$ and $q_{2}$.

Following Dixit [14] and Singh and Vives [15], we suppose that the utility function used in this chapter is quadratic and can be given by,

$$
U\left(q_{1}, q_{2}\right)=a\left(q_{1}+q_{2}\right)-\frac{b}{2}\left(q_{1}^{2}+2 q_{1} q_{2}+q_{2}^{2}\right)
$$

where $q_{1}, q_{2}$ are the quantity of goods produced by firm 1 and firm 2 , respectively. $a>0$ represents the maximum price of a unit's commodity, $b>0$ represents the amount of its price decreases when the price of the product increases by one unit.

Suppose that the budget constraint of consumer is,

$$
p_{1} q_{1}+p_{2} q_{2}=M
$$

where $p_{1}$ and $p_{2}$ denote the prices of goods produced by firm 1 and firm 2, respectively. And $M$ denotes the budget of the consumers on the product. The utility function of consumers is maximized under the budget constraint, and then the inverse demand function of the two firms can be obtained as,

$$
p_{i}=a-b\left(q_{1}+q_{2}\right), i=1,2
$$

This chapter discusses homogenous products, so here it is assumed that all these two players have the identical marginal cost. Therefore, the cost function of firm 1 and firm 2 are same and can be given by, $C(q)=c q$, where $c>0$ denotes the marginal cost of the goods and $a>c$ always holds. Then, the profits of firm $i, i=1,2$ can be obtained as follows,

$$
\pi_{i}=\left[a-b\left(q_{1}+q_{2}\right)-c\right] q_{i}, i=1,2
$$

In the real market, there are a lot of firms, who not only pursue their own profits but also take corporate social responsibility into account. A large number of 
empirical studies have shown how the introduced corporate social responsibility affects firm's performance, where we interpret corporate social responsibility as either consumer surplus (for short CS) or social welfare (for short SW). In this chapter we take CS into account to analyze which firms have an incentive to exhibit corporate social responsibility as a means of maximizing their profits in a Cournot competition. Based on the above assumptions and the definition of consumer surplus, CS can be written as,

$$
C S=\int_{p}^{a} \frac{a-\bar{p}}{b} d \bar{p}=\frac{b\left(q_{1}+q_{2}\right)^{2}}{2}
$$

where $\bar{p} \in(p, a)$ is the price variable.

According to the above assumptions, the objective function of the firm $i$ can be given as,

$$
O_{i}=\left(1-\alpha_{i}\right) \pi_{i}+\alpha_{i} C S, \quad 0 \leq \alpha_{i} \leq 1, \quad i=1,2
$$

where $\alpha_{i}$ represents the weight of the consumer surplus in the objective function of firm $i$, and $0 \leq \alpha_{i} \leq 1$ always holds. By substituting (4) and (5) into (6), the objection function of firm $i$ can be given by,

$$
O_{i}=\left(1-\alpha_{i}\right)\left[a-b\left(q_{1}+q_{2}\right)-c\right] q_{i}+\frac{1}{2} \alpha_{i} b\left(q_{1}+q_{2}\right)^{2}, \quad i=1,2
$$

And the first-order condition of the objection function (7) is given as,

$$
\frac{\partial O_{i}}{\partial q_{i}}=\left(1-\alpha_{i}\right)(a-c)+\left(3 \alpha_{i}-2\right) b q_{i}+\left(2 \alpha_{i}-1\right) b q_{j}, i, j=1,2, i \neq j
$$

It is now significant to specify the information set of both players regarding the objection functions, to determine the behaviors of the players with the change of time. We assume a discrete time $\left(t \in Z_{+}\right)$dynamic setting, where two firms with bounded rationality make decisions at the same time. That is, all firms do not have complete knowledge of their competitors' decisions and the market demands. So it can only use the local estimation of the steepest slope of the objection function at period $t$ to determine the output at period $t+1$. By following Bischi et al. [16] and Fanti et al. [17], the adjustment mechanism of quantities with the change of time of firm $i$ can be obtained as,

$$
q_{i}(t+1)=q_{i}(t)+v_{i} q_{i}(t) \frac{\partial O_{i}}{\partial q_{i}}
$$

where $v_{i}>0, i=1,2$ is an adjustment parameter of firm $i$. The firm $i$ will increase its output at period $t+1$, if $\frac{\partial O_{i}(t)}{\partial q_{i}(t)}>0$. But the firm $i$ will reduce its output at period $t+1$, if $\frac{\partial O_{i}(t)}{\partial q_{i}(t)}>0$. By substituting (8) into (9), we can get a two-dimensional map as,

$T:\left\{\begin{array}{l}q_{1}(t+1)=q_{1}(t)+v_{1} q_{1}(t)\left[\left(1-\alpha_{1}\right)(a-c)+\left(3 b \alpha_{1}-2 b\right) q_{1}(t)+\left(2 b \alpha_{1}-b\right) q_{2}(t)\right] \\ q_{2}(t+1)=q_{2}(t)+v_{2} q_{2}(t)\left[\left(1-\alpha_{2}\right)(a-c)+\left(3 b \alpha_{2}-2 b\right) q_{2}(t)+\left(2 b \alpha_{2}-b\right) q_{1}(t)\right]\end{array}\right.$

Since the output of a firm cannot be negative, the initial conditions of map $T$ belong to 


$$
F=\left\{\left(q_{1}, q_{2}\right): q_{1} \geq 0, q_{2} \geq 0, q_{1}+q_{2} \neq 0\right\}
$$

By setting $q_{i}(t+1)=q_{i}(t), i=1,2$ in system (10), the fixed points of the system are obtained. Besides the trivial equilibrium $E_{0}=(0,0)$, system (10) admits the following non-trivial fixed points (boundary equilibrium points),

$$
E_{1}=\left(0,-\frac{(a-c) A_{2}}{b B_{2}}\right), E_{2}=\left(-\frac{(a-c) A_{1}}{b B_{1}}, 0\right)
$$

and the only Nash equilibrium is

$$
E^{*}=\left(\frac{(a-c)\left(A_{2} C_{1}-A_{1} B_{2}\right)}{b\left(B_{1} B_{2}-C_{1} C_{2}\right)}, \frac{(a-c)\left(A_{1} C_{2}-A_{2} B_{1}\right)}{b\left(B_{1} B_{2}-C_{1} C_{2}\right)}\right)
$$

where $A_{1}=1-\alpha_{1}, A_{2}=1-\alpha_{2}, B_{1}=3 \alpha_{1}-2, B_{2}=3 \alpha_{2}-2, C_{1}=2 \alpha_{1}-1$ and $C_{2}=2 \alpha_{2}-1$. The positivity of $E_{i}(i=1,2)$ and $E^{*}$ is ensured by requiring $S=S_{1} \cup S_{2}$, where

$$
\left\{\begin{array}{l}
S_{1}=\left\{\left(A_{1}, A_{1}, B_{1}, B_{2}, C_{1}, C_{2}\right) \mid B_{1}<0, B_{2}<0, B_{1} B_{2}-C_{1} C_{2}>0, A_{2} C_{1}-A_{1} B_{2}>0, A_{1} C_{2}-A_{2} B_{1}>0\right\} \\
S_{2}=\left\{\left(A_{1}, A_{1}, B_{1}, B_{2}, C_{1}, C_{2}\right) \mid B_{1}<0, B_{2}<0, B_{1} B_{2}-C_{1} C_{2}<0, A_{2} C_{1}-A_{1} B_{2}<0, A_{1} C_{2}-A_{2} B_{1}<0\right\}
\end{array}\right.
$$

\section{Stability properties}

The local stability analyses of system (10) near the fixed points are too difficult to carry on. For the sake of analyzing the local stability of the system, we firstly let $\alpha_{1}=\alpha_{2}=\alpha$ in system (10). And the Jacobian matrix of map $T$ at any fixed point $\left(q_{1}, q_{2}\right)$ can be given as,

$J\left(q_{1}, q_{2}\right)=\left(\begin{array}{cc}1+v_{1}(1-\alpha)(a-c)+2 v_{1}(3 b \alpha-2 b) q_{1}+v_{1}(2 b \alpha-b) q_{2} & v_{1}(2 b \alpha-b) q_{1} \\ v_{2}(2 b \alpha-b) q_{2} & 1+v_{2}(1-\alpha)(a-c)+2 v_{2}(3 b \alpha-2 b) q_{2}+v_{2}(2 b \alpha-b) q_{1}\end{array}\right)$

Then all the equilibrium points are substituted into the Jacobian matrix (12). According to the characteristic values of the Jacobian matrix evaluated at each equilibrium, the type and stability of the equilibrium can be analyzed and the following results can be obtained.

Proposition 1. The equilibrium point $E_{0}$ is always an unstable node.

Proof. It is clear that the Jacobian matrix of map $T$, evaluated at the boundary equilibrium point $E_{0}$ can be written as,

$$
J\left(E_{0}\right)=\left(\begin{array}{cc}
1+v_{1}(1-\alpha)(a-c) & 0 \\
0 & 1+v_{2}(1-\alpha)(a-c)
\end{array}\right),
$$

The eigenvalues of $J\left(E_{0}\right)$ are given by $\lambda_{i}=1+v_{i}(1-\alpha)(a-c), i=1,2$. Since (11) holds and $v_{i}>0,(i=1,2)$ then $\lambda_{i}>1,(i=1,2)$ which implies that $E_{0}$ is an unstable node.

Proposition 2. $E_{1}$ is a saddle point, when $v_{2}<\frac{2}{(1-\alpha)(a-c)}$. And $E_{1}$ is an unstable node, when $v_{2}>\frac{2}{(1-\alpha)(a-c)}$. 
Proof. By substituting the equilibrium $E_{1}$ into (11), the Jacobian matrix of map $T$ evaluated at the boundary equilibrium point $E_{1}$ can be written as,

$$
J\left(E_{1}\right)=\left(\begin{array}{cc}
\lambda_{1}=1+v_{1}(1-\alpha)(a-c) \frac{\alpha-1}{3 \alpha-2} & 0 \\
-\frac{v_{2}(1-\alpha)(a-c)(2 \alpha-1)}{3 \alpha-2} & 1-v_{2}(1-\alpha)(a-c)
\end{array}\right),
$$

The eigenvalues of $J\left(E_{1}\right)$ are given by $\lambda_{1}=1+v_{1}(1-\alpha)(a-c) \frac{\alpha-1}{3 \alpha-2}$ and $\lambda_{2}=1-v_{2}(1-\alpha)(a-c)$. Since (11) holds and $v_{1}>0$, then $\lambda_{1}>1$ always meets. According to (11) and $v_{2}>0$, we can deduce that $\left|\lambda_{2}\right|<1$ if and only if $v_{2}<\frac{2}{(1-\alpha)(a-c)}$. That is, when $v_{2}<\frac{2}{(1-\alpha)(a-c)}$ meets, the equilibrium $E_{1}$ is a saddle point. Similarly, the equilibrium $E_{1}$ is an unstable node, when $v_{2}>\frac{2}{(1-\alpha)(a-c)}$.

Similar to the case of the equilibrium $E_{1}$, it can be also proved that $E_{2}$ is a saddle point when $v_{1}<\frac{2}{(1-\alpha)(a-c)}$, and $E_{2}$ is an unstable node when $v_{1}>\frac{2}{(1-\alpha)(a-c)}$.

For the purpose of research of the local stability near the Nash equilibrium, we should compute the Jacobian matrix evaluated at the Nash equilibrium $E^{*}$ as,

$J\left(q_{1}^{*}, q_{2}^{*}\right)=\left(\begin{array}{cc}1+v_{1}(1-\alpha)(a-c)+2 v_{1}(3 b \alpha-2 b) q_{1}^{*}+v_{1}(2 b \alpha-b) q_{2}^{*} & v_{1}(2 b \alpha-b) q_{1}^{*} \\ v_{2}(2 b \alpha-b) q_{2}^{*} & 1+v_{2}(1-\alpha)(a-c)+2 v_{2}(3 b \alpha-2 b) q_{2}^{*}+v_{2}(2 b \alpha-b) q_{1}^{*}\end{array}\right)$

It can be seen that the form of the Jacobian matrix is so complex. In order to simplify the calculation, let

$$
A=(1-\alpha)(a-c), B=b(3 \alpha-2), D=b(2 \alpha-1)
$$

Then the trace and the determinant of the Jacobian matrix evaluated at the Nash equilibrium $E^{*}$ can be given as,

$$
\begin{gathered}
\operatorname{Tr}=2+A\left(v_{1}+v_{2}\right)+\left(2 v_{1} B+v_{2} D\right) q_{1}^{*}+\left(2 v_{2} B+v_{1} D\right) q_{2}^{*} \\
\text { Det }=1+\left(v_{1}+v_{2}\right) A+v_{1} v_{2} A^{2}+\left(v_{2} D+v_{1} v_{2} A D+2 v_{1} B+2 v_{1} v_{2} A B\right) q_{1}^{*} \\
+\left(2 v_{2} B+2 v_{1} v_{2} A B+v_{1} D+v_{1} v_{2} A D\right) q_{2}^{*}+2 v_{1} v_{2} B D\left(\left(q_{1}^{*}\right)^{2}+\left(q_{2}^{*}\right)^{2}\right)+4 v_{1} v_{2} B^{2} q_{1}^{*} q_{2}^{*}
\end{gathered}
$$

According to Jury condition, if we substitute the specific mathematical expressions of $q_{1}^{*}, q_{2}^{*}$ into the above two equations, then the following set of inequalities can be gotten through a complex calculation,

$$
\left\{\begin{array}{l}
4-\frac{2 A B\left(v_{1}+v_{2}\right)-v_{1} v_{2} A^{2}(B-D)}{B+D}>0 \\
v_{1} v_{2} A^{2} \frac{B-D}{B+D}>0 \\
\frac{A B\left(v_{1}+v_{2}\right)-v_{1} v_{2} A^{2}(B-D)}{B+D}>0
\end{array}\right.
$$

Since all the equilibrium points are non-negative when the parameters meet $0 \leq \alpha<\frac{3}{5}, a>c$ and $v_{i}>0,(i=1,2)$. So we can get $A>0, B<0, B+D<0$ and $B-D<0$, then the set of inequalities (14) are equivalent to 


$$
\left\{\begin{array}{l}
4(B+D)-2 A B\left(v_{1}+v_{2}\right)+v_{1} v_{2} A^{2}(B-D)<0 \\
B\left(v_{1}+v_{2}\right)-v_{1} v_{2} A(B-D)<0
\end{array}\right.
$$

Then the stability region of the Nash equilibrium can be obtained by substituting $A, B$ and $D$ into inequalities (15), which are given as,

$$
\left\{\begin{array}{l}
4(5 \alpha-3)-2(1-\alpha)(a-c)(3 \alpha-2)\left(v_{1}+v_{2}\right)-v_{1} v_{2}(1-\alpha)^{3}(a-c)^{2}<0 \\
(3 \alpha-2)\left(v_{1}+v_{2}\right)+v_{1} v_{2}(1-\alpha)^{2}(a-c)<0
\end{array}\right.
$$

The stability condition of the Nash equilibrium gives a parameters region, in which the Nash equilibrium is always stable. For the sake of better analysis of the stability of the Nash equilibrium under different set of parameters, a useful tool called "two-dimensional bifurcation diagram" (also called 2-D bifurcation diagram) is employed. From (16), we can find that the stability region of the Nash equilibrium is related to the difference of parameters $a$ and $c$, that is $a-c$. So we only discuss the values of $a-c$, rather than the values of $a$ and $c$ in the rest of this chapter.

Figure 1 is a two-dimensional bifurcation diagram of system (10) with a set of fixed parameter $a-c, b$ and $\alpha$. Figure 1a is a two-dimensional bifurcation diagram when $a-c=2.1, \alpha=0.50608821$ and $b=2.00232504$. We can observe that there are two different routes to chaos, when the parameters are chosen as this set of parameters. The system enters chaos through flip bifurcation when the parameter $\left(v_{1}, v_{2}\right)$ passes through green, yellow and light green from the brown region to the
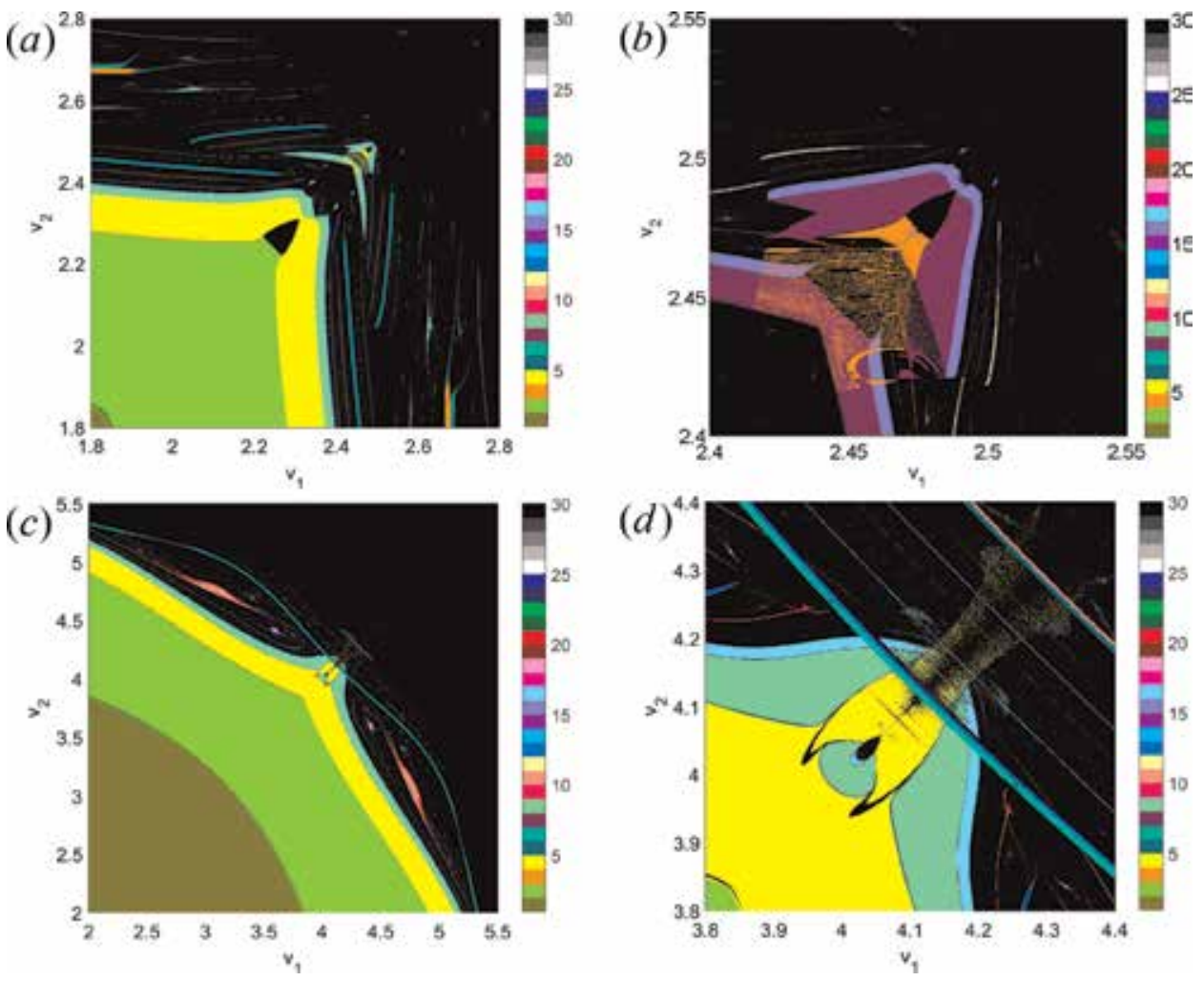

Figure 1.

$2 D$ bifurcation diagram in the $\left(v_{1}, v_{2}\right)$ or $\left(\alpha_{1}, \alpha_{2}\right)$ parameters plane. (a) $a-c=2.1, \alpha=0.50608821$, $b=2.00232504,(b)$ enlarged the square region in Figure 1a, $(c) a-c=0.88017028, \alpha=0.27445462$, $b=0.52714274$ and $(d)$ enlarged the square region in the Figure $1 c$. 
black region. It means that when the firms change their speed of adjustment according to the path, a periodic fluctuation of system (10) will happen. That is, the period motion will increase exponentially until it enters chaos. And the chaotic behavior of this system can be understood as the confusion of the market competition, and one of the two firms may be even out of the market with an increasing speed of adjustment. But if the parameter passes through the green region from the brown region to the black region, the system will first undergo a flip bifurcation, and then enters quasi-period motion through a Neimark-Sacker bifurcation. The system enters quasi-period from period-2 when firms determine their speed of adjustment along this path. Figure $\mathbf{1 b}$ is a partial amplification of Figure 1a, and we can observe that there are many scattered points of different colors, which is caused by the coexistence of multi-attractors with different period.

Figure 2a shows the coexistence of attractors with the parameters chosen as $v_{1}=2.44$, and $v_{2}=2.45$, where the scatter points are shown in Figure $\mathbf{1} \mathbf{b}$. we can observe a period- 6 cycle coexisting with a period- 4 cycle. Figure $1 \mathrm{c}$ is the twodimensional bifurcation diagram, where the fixed parameters are given as $a-c=0.88017028, \alpha=0.27445462$ and $b=0.52714274$. At this set of parameters, the system enters chaos through a flip bifurcation. Figure 1d is a partial enlargement of Figure 1c. Similarly, the parameter space of Figure 1d is also chosen according to the area with scattered points of Figure 1c. Figure $\mathbf{2 b}$ shows the coexisted attractors and their basins of attraction at this set of parameters. We can observe that there are three attractors coexisting.

Figure 3 shows a series of two-dimensional bifurcation diagram under different parameters. It shows a very beautiful gallery, from which we can enjoy the system (10) with full complex dynamics phenomenon. We can observe from Figure 3 that the difference between the maximum price of the unit product $a$ and the marginal cost $c$ affects the size of the stable region. The weight of the consumer surplus $\alpha$ affects the shape of the two-dimensional bifurcation diagram, while the parameter $b$ almost has hardly effect on the two-dimensional bifurcation of the system.

Therefore, the game can be balanced more quickly by reducing the difference $a-c$. In Figure 3a, it can be observed that the chaotic area surrounded by the period- 4 area is like a "hand" and the 8-period area is like a small "bottle" raised by the beautiful hand. It is observed that the shape of Figure $3 \mathbf{b}$ is similar to Figure $3 \mathbf{a}$ due to a tiny adjustment of parameter $\alpha$. Since the difference between the parameters $a$ and $c$ is reduced, the stability area of the Nash equilibrium becomes larger.

Figure $3 c$ is like a "volcanic eruption." It can be observed that there is an inward
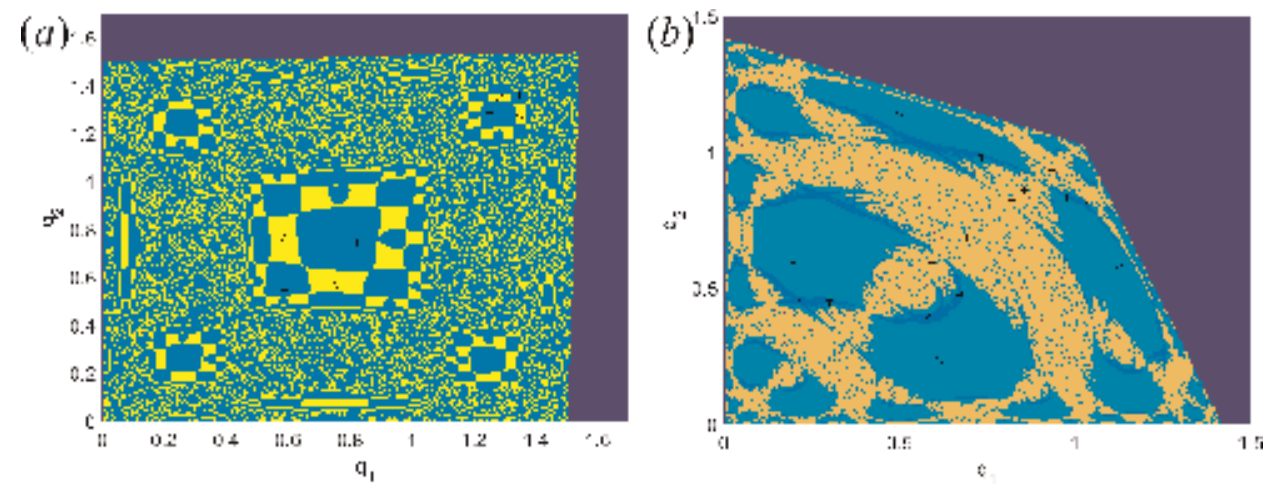

Figure 2.

(a) A period-8 cycle coexists with a period-8 cycle with $a-c=2.1, \alpha=0.50608821, b=2.00232504$, $v_{1}=2.44$, and $v_{2}=2.45$ and $(b)$ a attractor on the diagonal and a period-4 cycle coexists with a period-8 cycle with $a-c=0.88017028, \alpha=0.27445462, v_{1}=4.16, b=0.52714274$, and $v_{2}=4.13$. 

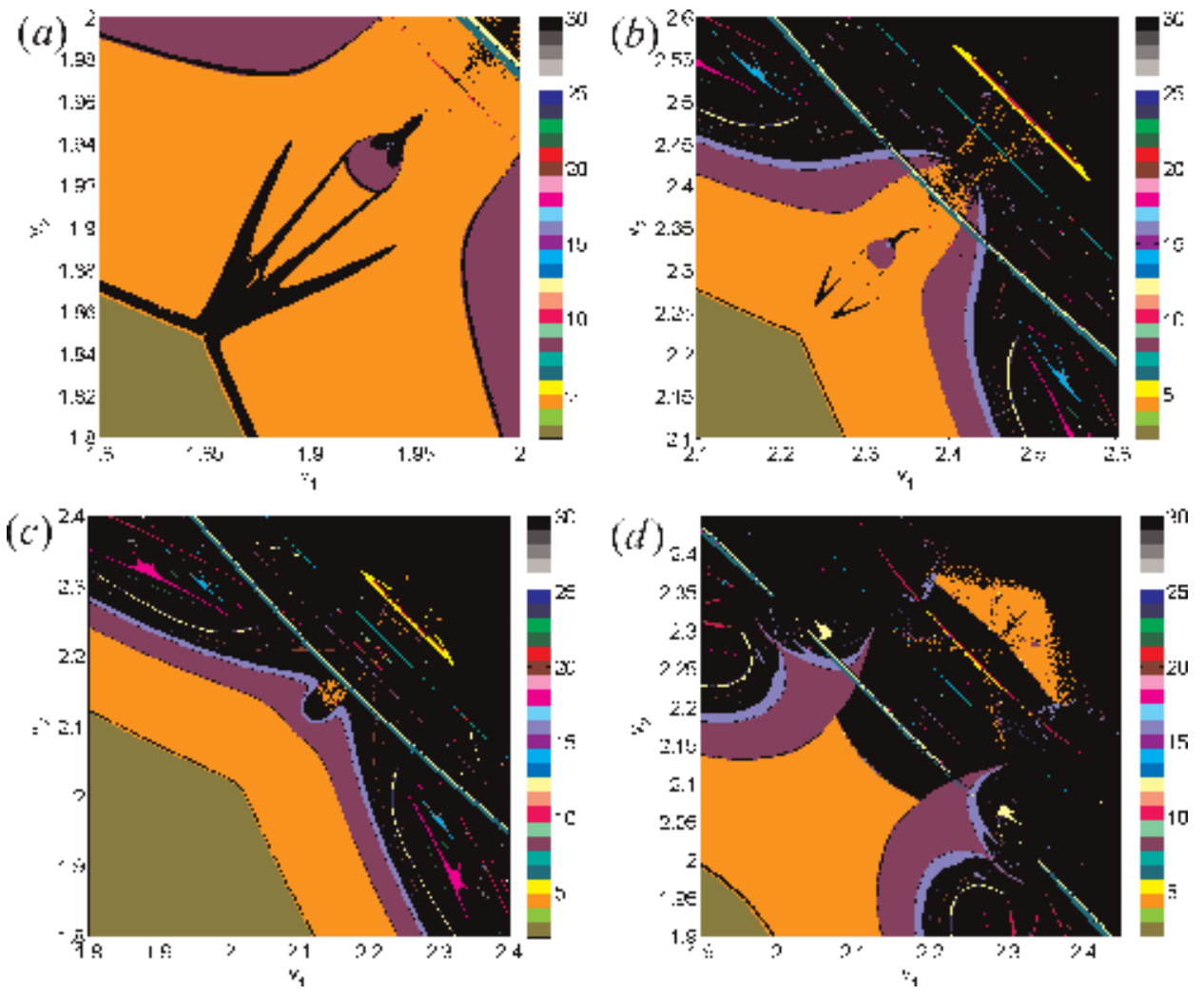

Figure 3.

$2 D$ bifurcation diagram in the $\left(v_{1}, v_{2}\right)$ parameters plane, $(a)$ with parameters $a-c=1.83, \alpha=0.278$, $b=0.0012$, (b) with parameters $a-c=1.52, \alpha=0.277, b=0.348,(c)$ with parameters $a-c=1.66$, $\alpha=0.27, b=0.19$ and $(d)$ with parameters $a-c=1.99, \alpha=0.39, b=0.22$.

cave in the diagonal that is like a "crater." As the parameters vary, the hole in Figure 3c continues to sink inward and become larger and larger. From Figure 3d we can see that the period-4 arrives at a quasi-periodic motion directly. Therefore, we should change the weight of consumer surplus $\alpha$ slightly in order to maintain the market fluctuations not fierceness.

\section{Global dynamics and synchronization}

The type and stability of the equilibrium points have been analyzed as above. And the boundary equilibrium $E_{1}$ and the boundary equilibrium $E_{2}$ are in symmetrical positions with respect to the main diagonal line $\Delta=\left\{\left(q_{1}, q_{2}\right) \in R_{+}^{2}: q_{1}=q_{2}\right\}$. It is also clear that the unique Nash equilibrium $E^{*}$ of system (10) is located on the main diagonal $\Delta$. So we mainly study the dynamical behavior of the system on the diagonal. We choose the initial conditions near the diagonal, and the phenomenon via finite iteration back to the diagonal is called synchronization. The synchronization of chaotic systems was quite interesting and unexpected. In fact, due to the nonlinear system usually has sensitive dependence on initial conditions, a property which implies that the slightly change of initial conditions will lead to an exponential difference between the trajectories of two identical systems, making it impossible for two separated and even identical systems to synchronize. Therefore, the small coupling between two chaotic oscillators makes the system asymptotically converge to same trajectory, which is worth studying. 
Subsequently, we assume that both firms have the same speed of adjustment. It means that the latter discussion is based on $v_{1}=v_{2}=v$ and $\alpha_{1}=\alpha_{2}=\alpha$. In this case the two players are identical; the system $T$ can then be rewritten as follows,

$$
T^{\prime}:\left\{\begin{array}{l}
q_{1}(t+1)=q_{1}(t)+v q_{1}(t)\left[(1-\alpha)(a-c)+(3 b \alpha-2 b) q_{1}(t)+(2 b \alpha-b) q_{2}(t)\right] \\
q_{2}(t+1)=q_{2}(t)+v q_{2}(t)\left[(1-\alpha)(a-c)+(3 b \alpha-2 b) q_{2}(t)+(2 b \alpha-b) q_{1}(t)\right]
\end{array}\right.
$$

It can be proved that the map $T^{\prime}$ has symmetry property, i.e., there exists a map $S:\left(q_{1}, q_{2}\right) \rightarrow\left(q_{2}, q_{1}\right)$, which makes $T^{\prime} \circ S=S \circ T^{\prime}$. The symmetry property of the map $T^{\prime}$ implies that the diagonal $\Delta$ is a one-dimensional sub-manifold of system (17), i.e., $T^{\prime}(\Delta) \subseteq \Delta$. However, the phenomenon of synchronization occurs when the diagonal $\Delta$ is invariant one-dimensional submanifold of the system (17). Therefore, the phenomenon of synchronization of the system can be analyzed by studying the invariant set. We can also use critical curve and noninvertible map to describe the global dynamical behaviors of a 2-dimensional map.

\subsection{Critical curve and noninvertible map}

We divide the discrete dynamical system into invertible and noninvertible. The invertible discrete dynamical system refers that an image $\left(q_{1}{ }^{\prime}, q_{2}{ }^{\prime}\right)$ of the map $T^{\prime}$ is correspond to the only preimage $\left(q_{1}, q_{2}\right)$. The noninvertible discrete dynamical system implies that the map $T^{\prime}$ is multi-valued, i.e., the image of $T^{\prime}$ has more than one preimages. In a noninvertible discrete dynamical system, the curve that divides the phase space into regions with a different number of rank-1 preimages is called critical curve, denoted by $L C$. And the regions can be represented by $Z_{i},(i \in N)$. For example, a point belonging to area $Z_{0}$ has no preimage and a point belonging to area $Z_{2}$ has two preimages. Let us denote the rank-1 preimages of critical curve $L C$ under map $T^{\prime}$ as $L C_{-1}$. The set $L C$ is the 2-dimensional generalization of the critical value or local extremes of 1-dimensional noninvertible map. Its preimages $L C_{-1}$ are corresponding to local extreme point (critical point) in the one-dimensional noninvertible map. Since the map (17) is a continuously differentiable map, $L C_{-1}$ belongs to the locus of points where the Jacobian determinant of $T^{\prime}$ vanishes, i.e., $L C_{-1}=\left\{\left(q_{1}, q_{2}\right) \in R^{2} \mid \operatorname{det} D T^{\prime}=0\right\}$. In this case, curve $L C_{-1}$ can be determined by the following equation,

$1+2 v A+v^{2} A^{2}+\left(v D+v^{2} A D+2 v B+2 v^{2} A B\right)\left(q_{1}+q_{2}\right)+2 v^{2} B D\left(q_{1}^{2}+q_{2}^{2}\right)+4 v^{2} B q_{1} q_{2}=0$

$L C$ is the rank-1 image of $L C_{-1}$ under map $T^{\prime}$. That is, $L C=T^{\prime}\left(L C_{-1}\right)$. The noninvertible properties play a significant role in analyzing the global behavior of a nonlinear discrete dynamical model. So the critical curve is a powerful tool for us to study these complex structures. Using the segment of critical curve as well as their preimages of any rank, and we will get the boundary of the basins of attraction as shown in Figure 10f.

\subsection{Invariant sets}

The dynamics of the system on the diagonal is studied by analyzing the invariant sets. Firstly, we can prove that the coordinates are invariant sets of map $T^{\prime}$. Let $q_{2}(t)=0$, then we can obtain $q_{2}(t+1)=0$, and the first equation of (17) can be rewritten as, 


$$
q_{1}(t+1)=q_{1}(t)+v q_{1}(t)^{1\left[(1-\alpha)(a-c)+(3 b \alpha-2 b) q_{1}(t)\right]}
$$

It is easy to verify that the dynamics on the axis $q_{2}$ is also controlled by the map (19). It means that the system (17) can be regarded as a 1-dimensional map at the coordinate axes. The map (19) is topologically conjugate to the standard logistic map $x(t+1)=\omega x(t)(1-x(t))$ through a linear transformation, which is given as,

$$
q_{1}=\left[\frac{1+v(1-\alpha)(a-c)}{v(2 b-3 b \alpha)}\right] x
$$

and the parameter $\omega$ can be presented as $\omega=1+v(1-\alpha)(a-c)$. Thus the nonlinear dynamics of system (17) on the invariant axes can be analyzed through the standard logistic map.

It can also be proved that the diagonal $\Delta$ is an invariant set of system (17), i.e., the trajectory starting from the diagonal $\Delta$ will stay forever on it. Therefore, the dynamical behavior of system (17) can be analyzed through the map $T^{\prime}$ which is restricted to the diagonal. If we let $q_{1}=q_{2}=q$, then the dynamics generated by $T^{\prime}$ on the diagonal $\Delta$ can be analyzed through the following map,

$$
T_{\Delta}^{\prime}: q(t+1)=q(t)+v q(t)((1-\alpha)(a-c)+(5 b \alpha-3 b) q(t))
$$

Similarly, through the following linear transformation

$$
q=\left[\frac{1+v(1-\alpha)(a-c)}{v(3 b-5 b \alpha)}\right] y
$$

we can also prove that the map (21) is topologically conjugate to the standard logistic map $y(t+1)=\mu y(t)(1-y(t))$, where

$$
\mu=\omega=1+v(1-\alpha)(a-c) .
$$

Through the standard logistic map, we can easily analyze the dynamical behavior of the two-dimensional map $T^{\prime}$ on the diagonal $\Delta$. Under this situation, the Nash equilibrium $E^{*}$ of the system (10) is identical with the fixed point of map $T_{\Delta}{ }^{\prime}$. Since $\mu=1+v(1-\alpha)(a-c)$, we take different values of the bifurcation parameter $\mu$ of the logistic map, and Figure 4a gives different bifurcation curves of the system on the parameter plane $(\alpha, v)$. The flip bifurcation occurs when the system parameter $v$ equals $v=\frac{2}{(1-\alpha)(a-c)}$, and the Nash equilibrium $E^{*}$ loses its stability and forms a period-2 cycle around $E^{*}$. At $v=\frac{\sqrt{6}}{(1-\alpha)(a-c)}$, the period-2 cycle generates a period4 cycle after a flip bifurcation. When $\mu \approx 3.5699$, the standard period doubling cascade ends and the system enters chaos. When $v>\frac{3}{(1-\alpha)(a-c)}$, the general trajectory of the map $T_{\Delta}{ }^{\prime}$ is divergent.

As shown in Figure 4b, which the parameters is the same as Figure 4a, a twodimensional bifurcation diagram of the system with $v$ and $\alpha$ is obtained. Since it has been proved that the map $T_{\Delta}{ }^{\prime}$ is topologically conjugate to the logistic map, we can find that the bifurcation curves of the two graphs are the same. In Figure 4a, the curve $C_{1}$ is correspond to the equation $v=\frac{2}{(1-\alpha)(a-c)}$, and the region below it represents the set of points of $v$ and $\alpha$ at $1<\mu<3$. In this region, the fix point is stable. That is, the synchronization trajectory converges to the Nash equilibrium point. In Figure $\mathbf{4 b}$, it corresponds to the period- 1 region below the green region. When the point above the curve $C_{2}$ passes through the curve $C^{\prime}$ in Figure $4 \mathbf{a}$, the system goes into chaos through a period doubling cascade. In Figure $\mathbf{4 a}$, the curve $C_{\infty}$ is correspond to the equation $v=\frac{3}{(1-\alpha)(a-c)}$, where the parameters $v$ and $\alpha$ of the 

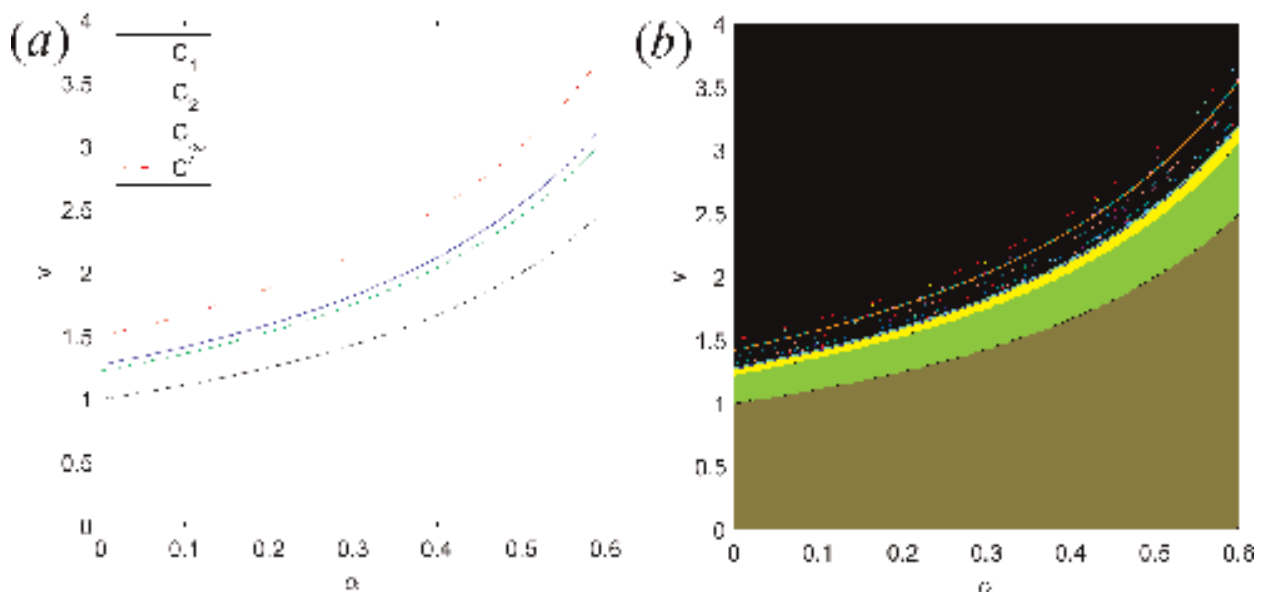

Figure 4 .

(a) Bifurcation curves on the parameter plane $(\alpha, v)$ related to the bifurcation values of map $T_{\Delta}$ for $a-c=2$ and $(b) 2 D$ bifurcation diagram in the parameter plane $(\alpha, v)$ for $a-c=2$ and $b=0.4$.

upper area lead to the divergence of the trajectory, corresponding to the black area in Figure $\mathbf{4 b}$. Through the above analysis, the following proposition can be derived,

Proposition 3. If we let $v_{1}=v_{2}=v$, the parameters $a, b$ and $c$ are fixed for system (17). Then, a threshold $\alpha^{\prime}=1-\frac{3}{v(a-c)}$ of the weight of consumer surplus $\alpha$ or a threshold $v^{\prime}=\frac{3}{(1-\alpha)(a-c)}$ of the speed of adjustment $v$ does exist such that synchronized trajectories of the system (17) are divergent when $\forall \alpha \in\left[0, \alpha^{\prime}\right)$ or $\forall v \in\left(v^{\prime},+\infty\right)$.

In order to analyze the effect of any slight perturbation of one parameter on the system, we study the transverse stability of an attractor $A$ of map $T^{\prime}$. And the Jacobian matrix of map $T^{\prime}$ on the diagonal can be obtained as follow,

$J(q, q)=\left(\begin{array}{ll}1+v(1-\alpha)(a-c)+v q(8 b \alpha-5 b) & v q(2 b \alpha-b) \\ v q(2 b \alpha-b) & 1+v(1-\alpha)(a-c)+v q(8 b \alpha-5 b)\end{array}\right)$

Then, the characteristic values of the Jacobian matrix $J(q, q)$ evaluated at any point on the diagonal are given by,

$$
\begin{aligned}
& \lambda_{\|}=1+v(1-\alpha)(a-c)+v q(10 b \alpha-6 b) \\
& \lambda_{\perp}=1+v(1-\alpha)(a-c)+v q(6 b \alpha-4 b)
\end{aligned}
$$

where the corresponding eigenvectors are $(1,1)$ and $(1,-1)$, respectively. And the eigenvalue $\lambda_{\|}$is related to the invariant manifolds on the diagonal.

It is assumed that a period- $k$ cycle $\{(q(1), q(1)),(q(2), q(2)), \cdots,(q(k), q(k))\}$ embedded into the invariant set $\Delta$ of the map $T^{\prime}$ is correspond to the cycle $\{q(1), q(2), \cdots, q(k)\}$ of the map $T_{\Delta}{ }^{\prime}$ when the synchronized phenomenon occurs, the two multipliers are given as,

$$
\begin{aligned}
& \lambda_{\|}^{(k)}=\prod_{i=1}^{k}\left(1+v(1-\alpha)(a-c)+v(10 b \alpha-6 b) q_{i}\right) \\
& \lambda_{\perp}^{(k)}=\prod_{i=1}^{k}\left(1+v(1-\alpha)(a-c)+v(6 b \alpha-4 b) q_{i}\right)
\end{aligned}
$$


Since the stability conditions of the period- $k$ cycle on the diagonal $\Delta$ of system (17) is same with the one-dimensional map $T_{\Delta}{ }^{\prime}$, here we only study the transverse stability of the one-dimensional map $T_{\Delta}{ }^{\prime}$. Under this situation, the transverse eigenvalue evaluated at the Nash equilibrium point $E^{*}$ is given by

$$
\lambda_{\perp}^{E^{*}}=1+\frac{v(a-c)(1-\alpha)^{2}}{5 \alpha-3}
$$

Through Eq. (27), we can draw the following conclusions directly. That is, when all the parameters satisfy $0<v(a-c)(1-\alpha)^{2}+5 \alpha<3$, the Nash equilibrium $E^{*}$ is transversely attractive.

As we know that an attractor $A$ of $T^{\prime}$ is asymptotically stable if and only if all the trajectories that belong to attractor $A$ are transversely attractive. To study the stability of the attractor, we can calculate its transverse Lyapunov exponent as,

$$
\Lambda_{\perp}=\lim _{n \rightarrow \infty} \sum_{i=0}^{n} \ln \left|\lambda_{\perp}(q(i))\right|
$$

where $q(0) \in A$ and $q(i)$ is a generic trajectory generated by the map $T_{\Delta}$. If the initial condition $q(0)$ belongs to a period- $k$ cycle, then $\Lambda_{\perp}=\ln \left|\lambda_{\perp}^{k}\right|$. In this case, if $\Lambda_{\perp}<0$, then the period- $k$ cycle is transversely stable. When the initial condition $q(0)$ belongs to a generic aperiodic trajectory embedded in the chaotic attractors, then the transverse Lyapunov exponent $\Lambda_{\perp}$ is the natural transverse Lyapunov exponent $\Lambda_{\perp}^{\text {nat }}$. Since many unstable cycles along the diagonal are embedded in the chaotic attractor $A$, a spectrum of transverse Lyapunov exponents can be determined by the inequality

$$
\Lambda_{\perp}^{\min } \leq \cdots \leq \Lambda_{\perp}^{\text {nat }} \leq \cdots \leq \Lambda_{\perp}^{\max }
$$

If all cycles embedded in $A$ are transversely stable $\left(\Lambda_{\perp}^{\max }<0\right)$ then $A$ is asymptotically stable in the Lyapunov sense. If some cycles embedded in the chaotic attractor $A$ are transversely unstable $\left(\Lambda_{\perp}^{\max }>0\right.$ and $\left.\Lambda_{\perp}^{\text {nat }}<0\right)$ then $A$ is not stable in the Lyapunov sense, but it is a stable Milnor attractor. So we can look for the Milnor attractors by transverse Lyapunov exponents.

Figure 5 gives the natural transverse Lyapunov exponent and the bifurcation diagram with the fixed parameter $v$ when $a-c=5.15, b=0.1911895$ and $\alpha=0.4$.
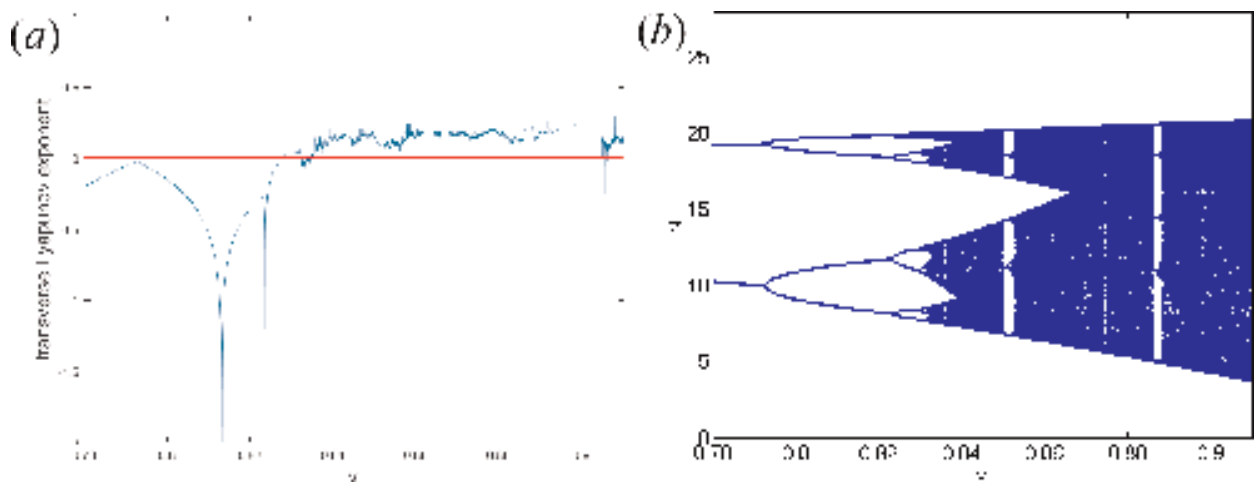

Figure 5.

Bifurcation diagram for the restriction of the map $T_{\Delta}$ to the invariant diagonal and the corresponding transverse Lyapunov exponent for $v \in[0.78,0.91]$ given $a-c=5.15, b=0.1911895$ and $\alpha=0.4$. 
Later, we will exhibit the attractors and their basins of attraction corresponding to different values $v$ under this set of parameters, and analyze the changes of attractors and their basins of attraction when the parameter $v$ varies.

\subsection{Global bifurcation and basins of attraction}

A closed invariant set $A$ is a attractor which means that it is asymptotically stable, i.e., a neighborhood $U$ of $A$ does exist such that $T^{\prime}(U) \subseteq U$ and $T^{\prime(m)}\left(q_{1}, q_{2}\right) \rightarrow A$ when $m \rightarrow \infty, \forall\left(q_{1}, q_{2}\right) \in A$. We also define a asymptotically stable invariant set as attractor. A basin of attraction may contain one or more attractors that may coexist with a set of repel points that produce either intermittent chaos or a blurry boundary. The basin of attraction of attractor $A$ is the set of those initial conditions that cause the trajectory to converge to A, i.e.,

$$
B(A)=\left\{\left(q_{1}(0), q_{2}(0)\right) \mid T^{m}\left(q_{1}(0), q_{2}(0)\right) \rightarrow A \text { as } m \rightarrow \infty\right\} .
$$

For the sake of analyzing the topological structure of the basin of attraction $B(A)$, we study the boundary of $B(A)$ firstly. Suppose that the map $T^{\prime}$ has a unique attractor $A$ at finite distance, let $\partial B(A)$ be the boundary of the basin $B(A)$, then it is also the boundary of the basin of infinity $B(\infty)$ generated by unbounded trajectories. Firstly, we take the dynamics of system (17) into account and restrict it to the invariant axis. When $v(1-\alpha)(a-c)<3$, if the initial conditions belong to the interval $\varepsilon_{i}=\left[0,0_{-1}^{i}\right],(i=1,2)$, according to map (17), we can obtain the bounded trajectories along the invariant axes, where $0_{-1}^{i},(i=1,2)$ is the rank-1 preimage of the origin. It has been obtained previously that the dynamical behavior of system (17) on the coordinate axis is governed by the map (19), so that $0_{-1}^{i},(i=1,2)$ can be computed by the following algebraic system

$$
q_{1}(t)+v q(t)\left[(1-\alpha)(a-c)+(3 b \alpha-2 b) q_{1}(t)\right]=0
$$

The result is given as,

$$
0_{-1}^{1}=0_{-1}^{2}=\frac{1+v(1-\alpha)(a-c)}{v b(3 \alpha-2)}
$$

Since $\varepsilon_{1}$ and $\varepsilon_{2}$ are the segments of the boundary $\partial B(A)$, and $\partial B(A)$ is also the boundary of the basin of infinity $B(\infty)$, their rank-k preimages $T^{(-k)}\left(\varepsilon_{i}\right),(i=1,2)$ also belong to $\partial B(A)$. We can compute the rank-1 preimages of a point $P=(p, 0) \in \varepsilon_{1}$ or $P=(0, p) \in \varepsilon_{2}$ that belongs to $\varepsilon_{i}=\left[0,0_{-1}^{i}\right], i=1,2$, according to the algebraic system as follows

$$
\left\{\begin{array}{l}
q(t)+v q(t)[(1-\alpha)(a-c)+(3 b \alpha-2 b) q(t)]=p \\
q(t)+v q(t)[(1-\alpha)(a-c)+(3 b \alpha-2 b) q(t)]=0
\end{array}\right.
$$

We can easily obtain the rank-1 preimages of the origin, which are $O_{-1}^{1}=\left(0_{-1}^{1}, 0\right)$ and $O_{-1}^{2}=\left(0,0_{-1}^{2}\right)$, there are itself and $O_{-1}^{3}$ besides, i.e.,

$$
O_{-1}^{3}=\left(\frac{1+v(1-\alpha)(a-c)}{v b(5 \alpha-3)}, \frac{1+v(1-\alpha)(a-c)}{v b(5 \alpha-3)}\right)
$$

Through the discussion above, we can get the following propositions, 
Proposition 4. Let $1<v(1-\alpha)(a-c)<3$ and $\varepsilon_{i}=\left[0,0_{-1}^{i}\right], i=1,2$ be the segments of the coordinate axes $q_{i}, i=1,2$, then we can obtain the boundary of $B(A)$ as follow,

$$
\partial B(A)=\left(\bigcup_{k=0}^{\infty} T^{\prime(-k)}\left(\varepsilon_{1}\right)\right) \cup\left(\bigcup_{k=0}^{\infty} T^{\prime(-k)}\left(\varepsilon_{2}\right)\right)
$$

Basins of attraction may be connected or not. The connected basins of attraction are divided into simple connected and complex one, and the complex connected basins of attraction means the existence of holes. If $A$ is a connected attractor, the direct basin of attraction $D_{0}$ of $A$ is the largest connected area of the entire attractor domain $D$ containing $A$. The system (17) has the coexistence of attractors in a set of given parameters, the basin of attraction $D$ refers to the union of the domain of attraction of all attractors in such a situation.

Figure 6 shows the coexistence of attractors and their basins of attraction for given parameters $a-c=5.3, b=0.234$ and $v=0.85$. In Figure 6a, the parameter $\alpha$ is chosen as $\alpha=0.4$, there are two attractors coexisting, one is a Milnor attractor $A$ located on the diagonal and the other consisting of 4-piece chaos attractor is in symmetrical positions with respect to the diagonal, i.e., $F=\cup_{i=1}^{4} F_{i}$. The basin of attraction is composed of the union of the attractive domain of two attractors. The attractive domain of the Milnor attractor $A$ is the complex connected set, and the attraction domain of the attractor $F$ is non-connected set. And the boundary of the 4-piece chaos attractor is just contact with the critical curve, and it is because of this contact that the system undergoes a global bifurcation. Figure $\mathbf{6 b}$ is the attractor and the attractive basin at $\alpha=0.387275$ after the global bifurcation occurred in the Figure 6a. We can find 4 attractors coexisted in this figure the attractor $F$ in Figure 6a undergoes a global bifurcation and turns into 3 period- 4 cycles, and the attractor $A$ is also a Milnor attractor. The attraction domain of the period-4 cycle is composed of some complex connected sets being in symmetrical positions with respect to the diagonal. As is shown in Figure $\mathbf{6 b}$, there are many holes in the attracting domain, but it is a non-connected set. The basin of attraction of the Milnor attractor lying on the diagonal is still a complex connected set.

We have analyzed the global bifurcations that occur when the attractor's boundary contact to the critical curve, and we discuss the global bifurcation when the attractor contacts to the boundary of its basin of attraction. We also denote global bifurcation as "boundary crisis," the attractor is destroyed when it contacts to its basin of attraction. Figure 7 shows the coexistence of attractors and their basins
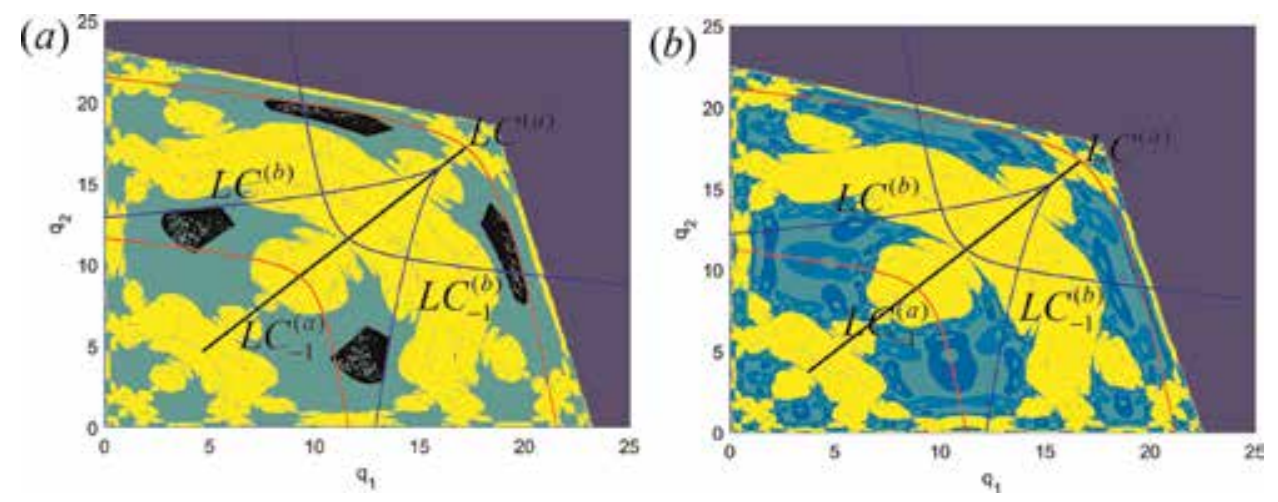

Figure 6.

Basin of attractions for parameters $a-c=5.3, b=0.234$ and $v=0.85$. (a) $\alpha=0.4$, a four-cyclic chaotic attractor coexists with a Milnor attractor on the diagonal and $(b) \alpha=0.387275$, a four-cyclic chaotic attractor has undergone a global bifurcation and three period-4 attractors coexist with a Milnor attractor. 

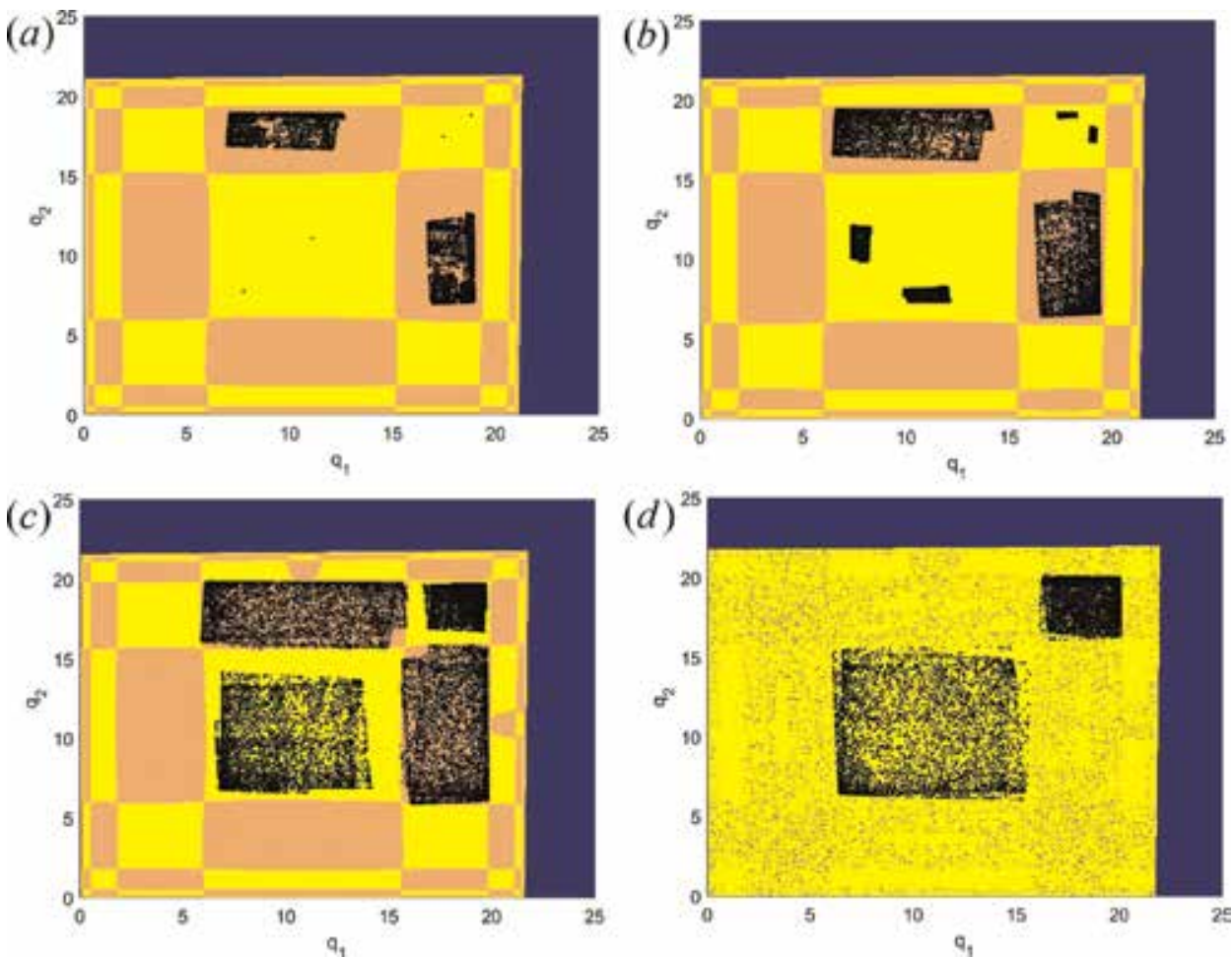

Figure 7.

Basin of attractions for parameters $b=0.4, \alpha=0.502335$ and $v=0.85$ (a) $a-c=6$, a two-piece chaotic attractor coexists with a period-4 cycle, $(b) a-c=6.1$, the 4-piece chaotic attractor coexist with a two-piece chaotic attractor, (c) $a-c=6.1896$, the 2-piece chaotic attractor formed by the a 4-piece chaotic attractor coexist with a two-piece chaotic attractor, and the many holes created by the global bifurcation and (d) $a-c=$ 6.26, a two-piece chaotic has a contact with its basin's boundary, and it is destroyed.

of attraction corresponding to the parameter $a-c$, when the parameters are chosen as $\alpha=0.5023335, b=0.4$ and $v_{1}=v_{2}=0.85$. We can see that as the difference between the maximum price $a$ of a unit commodity and the marginal cost c increases, a period-4 cycle turns into 4-piece chaos attractor being in symmetrical positions with respect to the diagonal and finally merges into 2-piece chaos attractor. However, the 2-piece attractor being in symmetrical positions with respect to the diagonal grows larger as the parameter $a-c$ increasing, until it contacts to its basin's boundary, and eventually occurs a global bifurcation, causing itself and its basin are destroyed until it disappears. We can also see its "ghost" in Figure 7d. This means that trajectories of the initial conditions that belong to the basin of attraction spend a long number of steps in the region occupied by the former attractor before converging to the other attractor. Figure 8 is the bifurcation diagram of the system at this set of parameters, and the bifurcation parameter is chosen as $a-c$.

Figures 6 and 7 give two different global bifurcations, such bifurcations which can be restored clearly by numerical simulation method only. With the set of parameters in Figure 9 being identical to Figure 4, we select different speed of adjustment to analyze the change of attractors and their basins of attraction. We can observe that as the speed of adjustment changes from 0.79 to 0.9 , the period- 4 cycle being in symmetrical positions with respect to the diagonal of the system generates smooth limit cycle via a Neimark-Sacker bifurcation, as shown in Figure $\mathbf{9 b}$, and the limit cycle becomes non-smooth gradually, and finally forms four-piece chaotic attractor, as shown in Figure 9d. The basin of attraction shrinks as the speed of adjustment $v$ increasing. It is implied that when both firms choose a lower speed of 
adjustment, they can reach the balance easily in the game. However, the period2 cycle embedded in the diagonal becomes a period- 4 cycle, period- 8 cycle, etc. That is, a flip-bifurcation happens. And finally a Milnor attractor forms with the increasing speed of adjustment. Its basin of attraction increases with the increasing speed of adjustment gradually. In the bifurcation diagram of Figure 5b, we can observe the process of entire bifurcation process.

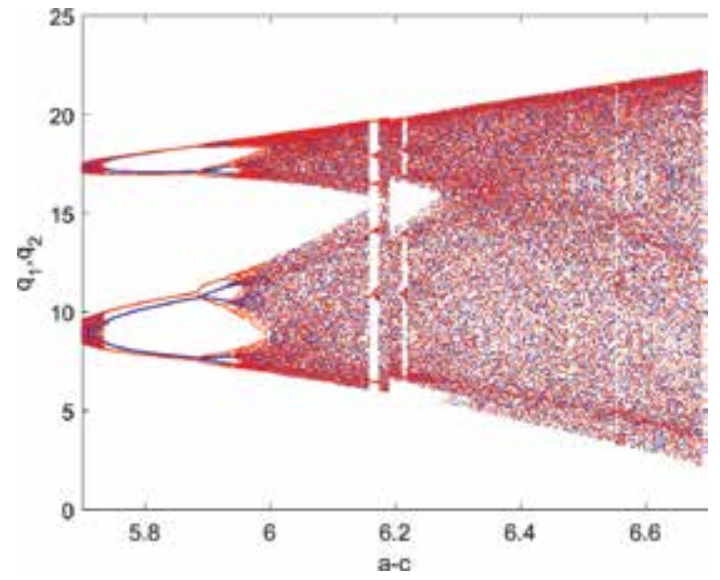

Figure 8.

One-dimensional bifurcation diagram with respect to $a-c$ for the set of parameters in Figure 7.
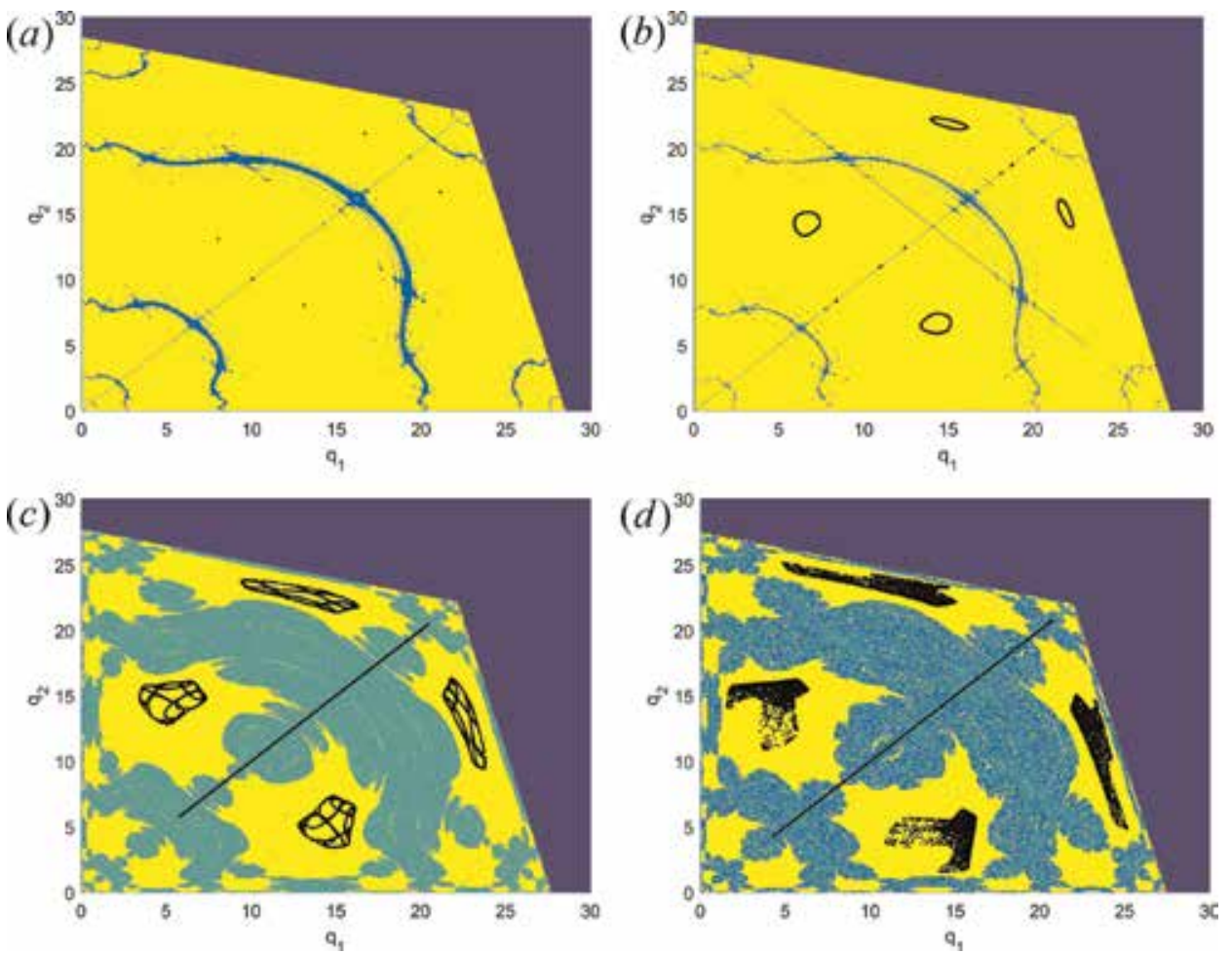

Figure 9.

Basin of attractions for parameter $a-c=5.15, b=0.1911895$ and $\alpha=0.4$. (a) $v=0.79$, an attracting four-period cycle coexists with the two-period cycle on the diagonal, $(b) v=0.83$, a period- 4 cycle has undergone a Neimark-Sacker bifurcation and an attractor formed by four smooth curves coexist with a period-8 cycle, (c) $v=0.872$, a four-cyclic chaotic attractor formed by the four smooth curves coexists with the Milnor attractor and $(d) v=0.9$, a four-piece chaotic attractor exists outside the diagonal. 


\subsection{Synchronization}

In this section we study the formation mechanism of the synchronization trajectories. The trajectories starting from different initial conditions return to the diagonal eventually, i.e., $q_{1}(0) \neq q_{2}(0)$. A $t^{*}$ does exist such that $q_{1}(t)=q_{2}(t)$ when $t>t^{*}$, and we define such trajectories as synchronization. However, when the diagonal $\Delta$ is an invariant sub-manifold, synchronized dynamics occur. We have proved that the map $T^{\prime}$ can be obtained by two identical one-dimensional coupling maps, and the synchronization trajectory can be controlled by a map $T_{\Delta}{ }^{\prime}$ which is topologically conjugate to the standard logistic map. When we choose the
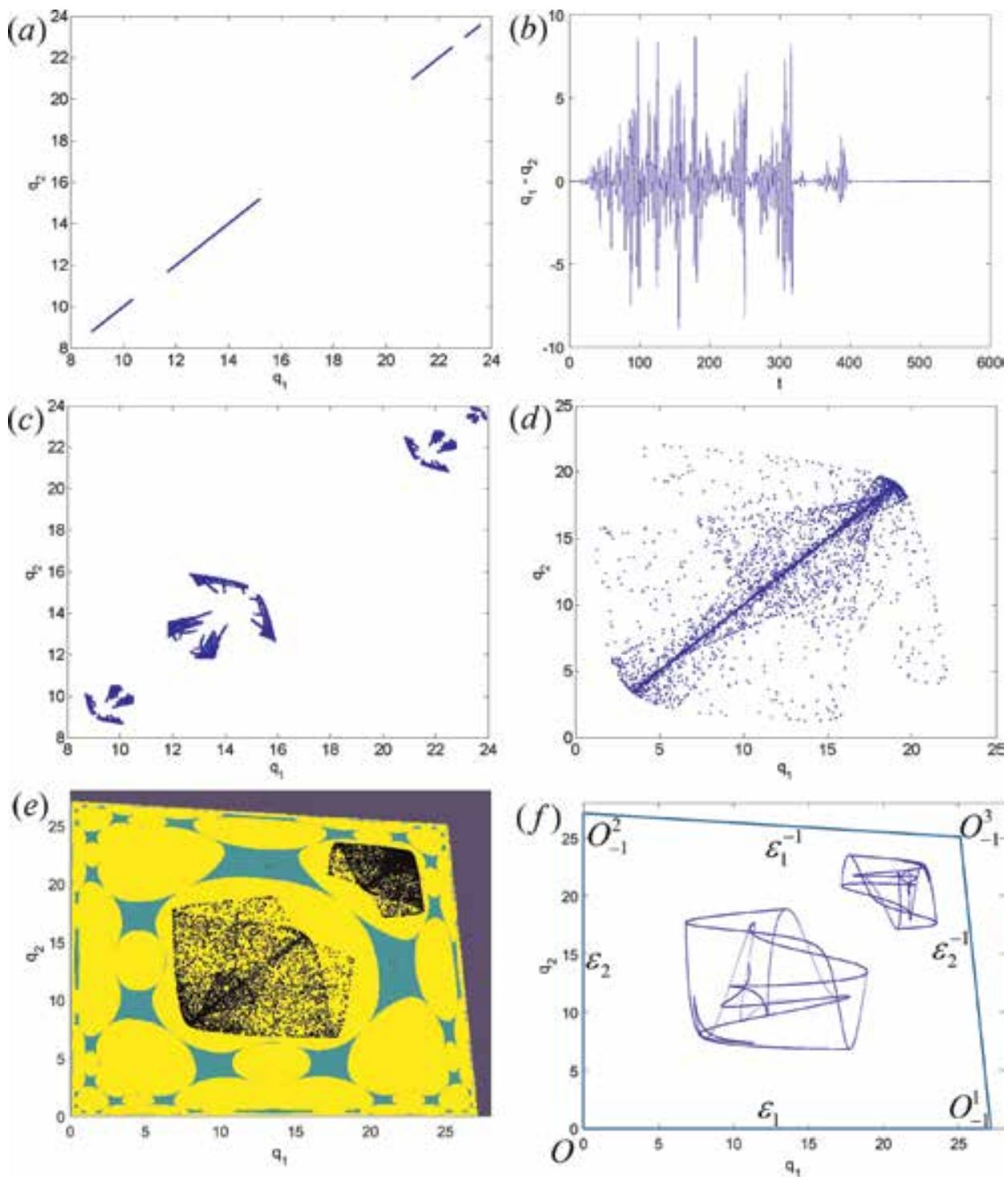

Figure 10.

Parameter values are chosen as $v=1, a-c=5$ and $b=0.234$. (a) Four-piece Milnor attractor of system $T$ belonging to the diagonal for $\alpha=0.48365$, (b) the displacement $q_{1}-q_{2}$ versus time for the same parameters as in (a), (c) $\alpha=0.485092$, a 16-cyclic chaotic attractor is in symmetrical positions with respect to the diagonal, (d) $\alpha=0.437609$, a trajectory in the phase space $\left(q_{1}, q_{2}\right)$ whose transient part is out of diagonal that synchronizes along the Milnor attractor in the long run, $(e) \alpha=0.476955$, a two-cyclic chaotic attractor coexists with a period-2 cycle and $(f)$ boundary of the chaotic area obtained by $\partial A=\cup_{k=1}^{6} T^{(k)}(\Gamma)$. 
parameters as $v=1, a-c=5$ and $b=0.234$, the weight $\alpha$ varies, and we can observe that the dynamic behavior of system is controlled by the attractor on the diagonal in Figure 10. When $\alpha=0.4837015$, we can observe a Milnor attractor in Figure 10a. This means that cycle embedded in the diagonal are transversely unstable and blowout phenomenon occurs when the trajectory is near diagonal. The trajectory converges to the unique Milnor attractor embedded in the diagonal after experiencing a long transient. Figure $10 \mathbf{b}$ shows that the evolution of $q_{1}-q_{2}$ versus time and synchronization is observed after a long transient. This is a typical on-off intermittency phenomenon. We can observe 16-piece chaos attractor being in symmetrical positions with respect to the diagonal in Figure 10c, when $\alpha$ increases to 0.485092 . Figure 10d shows a chaotic attractor when $\alpha$ decreases to 0.437609 , then synchronization occurs. As shown in Figure 10f, we adopt the trial-and-error method, with suitable part of $L C_{-1}$ taken as the starting part of $\Gamma=A \cap L C_{-1}$ to obtain the boundary of the chaotic attractor $A$ and the entire basin of attraction in

Figure 10e, i.e., the boundary of the chaotic attractor $A$ is $\partial A=\cup_{k=1}^{6} T^{\prime(k)}(\Gamma)$.

\section{Conclusion}

In this chapter, the nonlinear dynamics of a Cournot duopoly game with bounded rationality is investigated. Unlike the existing literature, we suppose that the two firms not only pursue profit maximization but also take consumer surplus into account. Meanwhile, the objection of firms is supposed as the weighted sum of profit and consumer surplus. Based on the theory of gradient adjustment, all the firms adjust the output of next period according to the estimation of "marginal goal." The existence and stability of fixed points are analyzed. It is found that the boundary equilibrium point is always unstable, no matter what the parameters of the system are satisfied. At the same time, with the two-dimensional bifurcation diagram as the tool, the stability of the Nash equilibrium is analyzed. We found that the Nash equilibrium will lose its stability when the speed of adjustment of firms is too large, which maybe lead the market into chaos. The stability region of the Nash equilibrium will be only affected by the weight of consumer surplus. And the parameters $a-c$ and $b$ have hardly effect on the stability region of the Nash equilibrium. Meanwhile, we found that the two-dimensional bifurcation diagram have a beautiful fractal structure, but there are also many scattered points which is due to the coexistence of multiple attractors of the system through numerical simulation. By selecting corresponding parameters in the two-dimensional bifurcation diagram with scattered points, we draw the corresponding basin of attraction, and found the model not only has two attractors coexistence phenomenon, but also has 3 , or even 4 attractors coexistence phenomenon.

Moreover, with the theory of invertible mapping and the critical curves of the system, the topological structure of basin of attraction is analyzed. By calculating the transverse Lyapunov exponent, the weak chaotic attractor of the system in the sense of Milnor is found, and the synchronization of the system is further studied. If we fix the other parameters of the system, and only change the weight of the firm to the consumer surplus, we can find on-off intermittency phenomenon and synchronization phenomenon. With the increasing of $\alpha$, the synchronization phenomenon is vanished and a 16-piece chaotic attractor being in symmetrical position with respect to the main diagonal is produced. Under another set of parameters, and the parameter $\alpha$ is chosen as the bifurcation parameter. Through numerical simulation, it can be found that when the critical curve contact with the boundary of the basin, a global bifurcation is obtained. The global bifurcation makes the basin of attraction 
of the attractor non connected. In addition, if we fixed parameters of the system, and change the values of the parameters $a-c$ only, we find another global bifurcation called "boundary crisis," i.e., when the attractor contact with its boundary of the basin of attraction, one of the attractors and its basin of attraction will be destroyed.

\section{Author details}

Wei Zhou ${ }^{1 *}$ and Tong $\mathrm{Chu}^{2}$

1 School of Mathematics and Physics, Lanzhou Jiaotong University, Lanzhou, China 2 School of Law, Zhejiang University of Finance and Economics, Hangzhou, China

*Address all correspondence to: wei_zhou@vip.126.com

\section{IntechOpen}

(C) 2019 The Author(s). Licensee IntechOpen. This chapter is distributed under the terms of the Creative Commons Attribution License (http://creativecommons.org/licenses/ by/3.0), which permits unrestricted use, distribution, and reproduction in any medium, provided the original work is properly cited. (c) BY 


\section{References}

[1] Friedman J. Oligopoly Theory. London: Cambridge University Press; 1983

[2] Elsadany AA. Dynamics of cournot duopoly game with bounded rationality based on relatve profit maximization. Applied Mathematics and Computation. 2017;294:253-263. DOI: 10.1016/j. amc.2016.09.018

[3] Andaluz J, Jarne G. Stability of vertically differentiated Cournot and Bertrand-type models when firms are boundedly rational. Annals of Operations Research. 2016;238:1-25. DOI: $10.1007 /$ s10479-015-2057-4

[4] Ma J, Guo Z. The influence of information on the stability of a dynamic Bertrand game. Communications in Nonlinear Science and Numerical Simulation. 2016;30:32-44. DOI: 10.1016/j.cnsns.2015.06.004

[5] Matsumoto A, Szidarovszky F, Yoshida H. Dynamics in linear Cournot duopolies with two time delays. Computational Economics. 2011;38:311. DOI: $10.1007 / \mathrm{s} 10614-011-9295-6$

[6] Gori L, Pecora N, Sodini M. Market share delegation in a nonlinear duopoly with quantity competition: The role of dynamic entry barriers. Journal of Evolutionary Economics. 2017;27:905-931

[7] Andaluz J, Jarne G. On the dynamics of economic games based on product differentiation. Mathematics and Computers in Simulation. 2015;113:16-27. DOI: 10.1016/j.matcom.2015.02.005

[8] Zhang Y, Zhou W, Chu T, et al. Complex dynamics analysis for a two-stage Cournot duopoly game of semi-collusion in production. Nonlinear Dynamics. 2018;91:819-835. DOI: 10.1007/s11071-017-3912-4

[9] Zhou J, Zhou W, Chu T, Chang YX, Huang MJ. Bifurcation, intermittent chaos and multi-stability in a two-stage Cournot game with R\&D spillover and product differentiation. Applied Mathematics and Computation. 2019; 341:358-378. DOI: 10.1016/j. amc.2018.09.004

[10] De Fraja G, Delbono F. Alternative strategies of a public enterprise in oligopoly. Oxford Economic Papers. 1989;41:302-311. DOI: 10.1093/ oxfordjournals.oep.a041896

[11] Matsumura T. Partial privatization in mixed duopoly. Journal of Public Economics. 1998;70:473-483. DOI: 10.1016/S0047-2727(98)00051-6

[12] Fujiwara K. Partial privatization in a differentiated mixed oligopoly. Journal of Economics. 2007;92:51-65. DOI: $10.1007 / \mathrm{s} 00712-007-0267-1$

[13] Elsadany AA, Awad AM. Nonlinear dynamics of Cournot duopoly game with social welfare. Electronic Journal of Mathematical Analysis and Applications. 2016;4:173-191

[14] Dixit AK. A model of duopoly suggesting a theory of entry barriers. Bell Journal of Economics. 1979;10: 20-32. DOI: $10.2307 / 3003317$

[15] Singh N, Vives X. Price and quantity competition in a differentiated duopoly. RAND Journal of Economics. 1984;15: 546-554. DOI: $10.2307 / 2555525$

[16] Bischi GI, Sbragia L, Szidarovszky F. Learning the demand function in a repeated Cournot oligopoly game. International Journal of Systems Science. 2008;39:403-419. DOI: 10.1080/00207720701792131

[17] Fanti L, Gori L, Sodini M. Nonlinear dynamics in a Cournot duopoly with relative profit delegation. Chaos, Solitons \& Fractals. 2012;45:1469-1478. DOI: 10.1016/j.chaos.2012.08.008 


\title{
Nonlinear Dynamical Regimes and Control of Turbulence through the Complex Ginzburg-Landau Equation
}

\author{
Joël Bruno Gonpe Tafo, Laurent Nana, Conrad Bertrand Tabi \\ and Timoléon Crépin Kofané
}

\begin{abstract}
The dynamical behavior of pulse and traveling hole in a one-dimensional system depending on the boundary conditions, obeying the complex Ginzburg-Landau (CGL) equation, is studied numerically using parameters near a subcritical bifurcation. In a spatially extended system, the criterion of Benjamin-Feir-Newell (BFN) instability near the weakly inverted bifurcation is established, and many types of regimes such as laminar regime, spatiotemporal regime, defect turbulence regimes, and so on are observed. In finite system by using the homogeneous boundary conditions, two types of regimes are detected mainly the convective and the absolute instability. The convectively unstable regime appears below the threshold of the parameter control, and beyond, the absolute regime is observed. Controlling such regimes remains a great challenge; many methods such as the nonlinear diffusion parameter control are used. The unstable traveling hole in the onedimensional cubic-quintic CGL equation may be effectively stabilized in the chaotic regime. In order to stabilize defect turbulence regimes, we use the global time-delay auto-synchronization control; we also use another method of control which consists in modifying the nonlinear diffusion term. Finally, we control the unstable regimes by adding the nonlinear gradient term to the system. We then notice that the chaotic system becomes stable under strong nonlinearity.
\end{abstract}

Keywords: Benjamin-Feir-Newell instability, subcritical bifurcation, complex Ginzburg-Landau equation, unstable traveling hole

\section{Introduction}

Many complex systems evolve in a non-equilibrium environment. Further out of the equilibrium [1], these systems tend to display progressively more complicated dynamics. The non-chaotic patterned state and spatiotemporal chaos are observed in the system. In the domain of the envelope equations, the quintic complex Ginzburg-Landau (CGL) equation is appropriate to obtain stable localized solutions (pulses, holes) $[1,2]$. Among physical applications of the quintic CGL equation, one 
can mention binary fluid convection [3], spiral waves in the Couette-Taylor flow between counterrotating cylinders [4], wave propagation in nonlinear optical fibers with gain and spectral filtering [5], the oscillatory chemical reaction [6], hydrodynamic turbulence [7], chemical turbulence [8,9], and electrical turbulence in the cardiac muscle [10]. Our work focuses on two types of systems: the spatially extended system and the finite system. In the case of spatially extended systems, we use as initial conditions a traveling-hole solution with periodic boundary conditions [11-15]. All the dynamical regimes obtained during our work are summarized in a phase diagram. In the case of the finite domain, we use as initial condition a pulse solution. Wave patterns are described by CGL equation in which the amplitude of the wave pattern vanishes at the lateral boundaries of the domain in order to retrieve numerically some coherent structures observed experimentally, in the case of absolute or convective instabilities [16-18].

Over the past decade, problems of chaos control and synchronization started to play a central role in the studies of chaotic dynamics [19] in many different areas such as chemistry [20], laser physics [21], electronic circuits [22], plasma [23], and mechanical systems. Since the pioneering work of Ott et al. [24] on the control of low-dimensional chaos in nonlinear systems based on Floquet theory, chaos control techniques have been well developed [25, 26]. Up to date, many control techniques have been suggested to control low-dimensional chaos by stabilizing unstable periodic orbits or fixed points. The realization of chaos control mainly includes feedback and non-feedback methods, both of which have advantages and disadvantages. Pyragas is one of the first to work on a delayed feedback loop called timedelay auto-synchronization (TDAS) [25]. Another part of our works is to control turbulence regimes observed, in particular the defect turbulence regime by employing the methods already successfully used in the cubic case, namely, the nonlinear diffusion technique [20], the feedback method [27], and the lower-order complex Ginzburg-Landau (LOCGL) equation [28-31]. The LOCGL equation which describes a system exhibiting a subcritical bifurcation to traveling waves must contain a quintic nonlinearity. It is obtained by adding nonlinear terms to the system. The effects of the nonlinear gradient terms are confirmed by using some indicators such as the Lyapunov exponent and the energy bifurcation diagram. Most of the results related to these different aspects are presented in the rest of this work.

\section{Dynamics of traveling hole in one-dimensional systems near subcritical bifurcation}

\subsection{Model description}

We consider a subcritical Hopf bifurcation, with a one-dimensional complex amplitude $A(x, t)$ and complex coefficients given by

$$
\frac{\partial A}{\partial t}=\left(1+i c_{1}\right) \frac{\partial^{2} A}{\partial x^{2}}+\mu A+\left(1-i c_{3}\right)|A|^{2} A-\left(1-i c_{5}\right)|A|^{4} A,
$$

where $c_{1}, c_{3}, c_{5}$, and $\mu$ are real constants. $\mu$ is the parameter control, and $t$ and $x$ represent, respectively, temporal and spatial variables. This equation is a paradigmatic model for the study of spatiotemporal dynamics [11]. It admits many different types of stable pulses [32] and hole-like [17] solutions. We have imposed on the complex amplitude the following boundary conditions: We consider a system in which we impose periodic boundary conditions: 
Nonlinear Dynamical Regimes and Control of Turbulence through the Complex...

DOI: http://dx.doi.org/10.5772/intechopen.88053

$$
A(x, t)=A(x+L, t),
$$

where $L$ is the length of the domain. These boundary conditions are realized in different extended systems, where the pattern amplitude vanishes near lateral boundaries. We have chosen as initial condition a hole solution given by [15]:

$$
A(x, 0)=\exp \left(i\left[q_{e x} x+(\pi / 2) \tanh (\gamma x)\right]\right) .
$$

The precise form of the initial condition is not important here as long as we have a one-parameter family of localized phase-gradient peaks. This is because the left moving and right moving coherent holes for fixed $c_{1}, c_{1}$, and $q_{e x}$ are each unique and have one unstable mode only. As $\gamma$ is varied, three possibilities can arise for the time evolution of the initial peak: evolution toward a defect. The nonzero $q_{e x}$ breaks the left-right symmetry and results in the differing periods of the left and right moving edge holes [15].

\subsection{Results of numerical simulation}

The parameters $c_{3}, \gamma$, and $q_{e x}$ were fixed at $c_{3}=0.50, \mu=1, \gamma=1.0$, and $q_{e x}=-0.03$. And, we have varied $c_{1}$ and $c_{5}$. This variation enabled us to identify, in the parameter space $\left(c_{1}, c_{5}\right)$, zones in which the patterns exhibit different behaviors. The different asymptotic phases observed are summarized in the state diagram of Figure 1, where the solid line corresponds to analytical results and represents the BFN line, while dashed lines are numerical ones. Dashed lines are obtained by varying $c_{1}$ and $c_{5}$ which gave us regions of different regimes. We have identified plane waves, spatiotemporal intermittency, phase turbulence, and weak turbulence and defect turbulence regimes.

\subsubsection{Plane wave regime}

The plane wave regime is a laminar state where no chaos is observed. The plane wave is localized below the BFN line in a zone called stable zone. The spatial profile

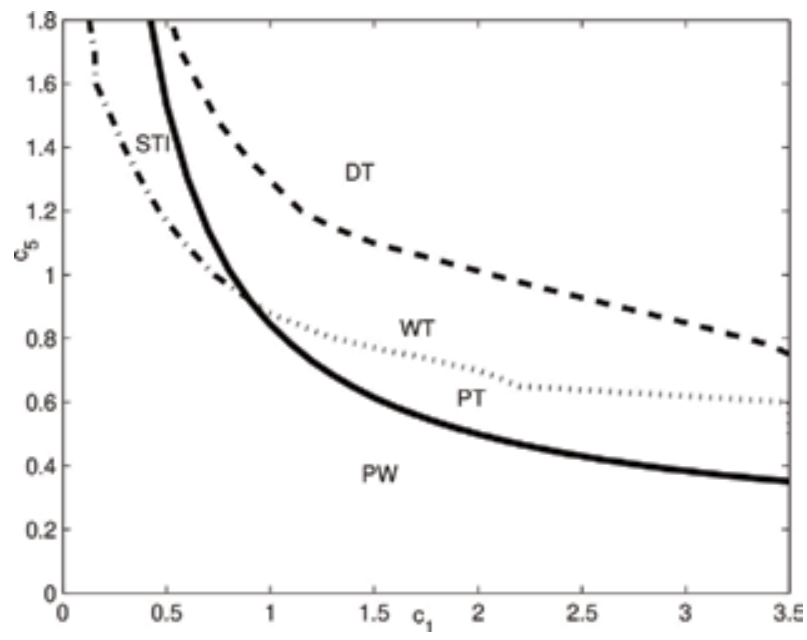

Figure 1.

Phase diagram of an initial traveling hole of the quintic CGLE showing different types of dynamical regimes: plane wave (PW), spatiotemporal intermittency (STI), phase turbulence (PT), weak turbulence (WT), defect turbulence $(D T) . c_{3}=0.5$, and $\mu=1.0$. 


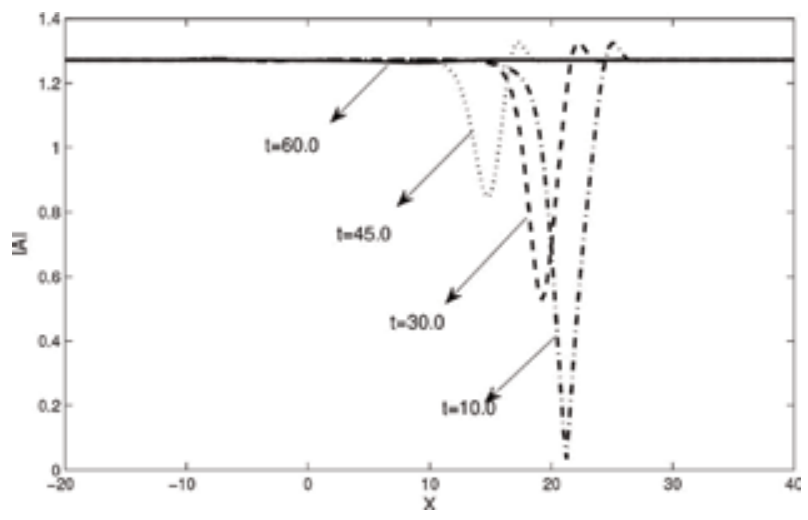

Figure 2.

Space-time plot of the wave amplitude $|A|$ in the case of the Benjamin-Feir stability for $c_{1}=2.5, c_{3}=2.0$, and $c_{5}=0.4$.

of the wave patterns in the plane wave regime is shown in Figure 2. We notice that by the growing of the time, the regime still stable, and laminar regime is observed.

\subsubsection{Spatiotemporal intermittency regime}

It consists of space-time regions of stable plane waves separated by localized objects evolving and interacting in a complex manner [33]. It represents a special scenario of transition to turbulence in extended systems: it is characterized by the coexistence of laminar (ordered) and turbulent (disordered) domains that occur randomly in different places of the system for the same values of the control parameters $[13,33]$. It has been observed in many experiments such as plane Couette flow, counterrotating Taylor-Couette flow, and Taylor-Dean system. In 1D extended systems, spatiotemporal intermittency has been observed in rectangular and annular Rayleigh-Bénard cells at large values of the Rayleigh number [15]. This spatiotemporal intermittency occurs via a subcritical bifurcation from purely laminar state, and the coexistence of two different stable states can be described phenomenologically using an amplitude equation derived from a Lyapunov function. We have plotted in Figure 3 the characteristic pattern of a spatiotemporal intermittency in which a global mode coexists with a chaotic attractor: the state consists of patches of plane waves, which are separated y various holes. Figure $\mathbf{3 b}$ and $\mathbf{c}$ shows in detail how a hole generates a phase defect and in turn generates two daughter holes close-up of the amplitude $|A|$ and close-up of the complex phase.

\subsubsection{Phase turbulence regime}

Just above the BFN line, the phase turbulence regime is observed (Figure 4). It is best defined by the absence of space-time defects. In this regime, the region is a weakly disordered one in which $|A(x, t)|$ remains away from zero. The absence of phase singularities implies that the "localwavenumber" given by

$$
\nu=\frac{1}{L} \int_{0}^{L} d x \partial_{x} \Psi(x, t),
$$

where $\Psi(x, t)$ is the phase, is a conserved quantity. A global wave number of the configuration can be defined as $k \equiv 2 \pi / L$. Chaos is very weak (see Figure 4 ). So the global phase difference becomes the constant of the motion and is conserved. This is corroborated by the flat shoulder of the spatial power spectrum of $|A(x, t)|$ at low 
Nonlinear Dynamical Regimes and Control of Turbulence through the Complex... DOI: http://dx.doi.org/10.5772/intechopen.88053

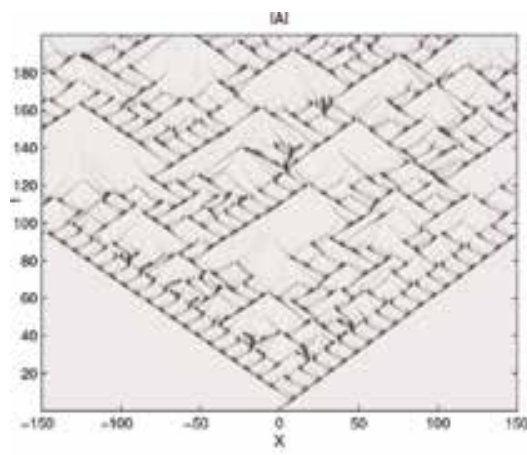

a)

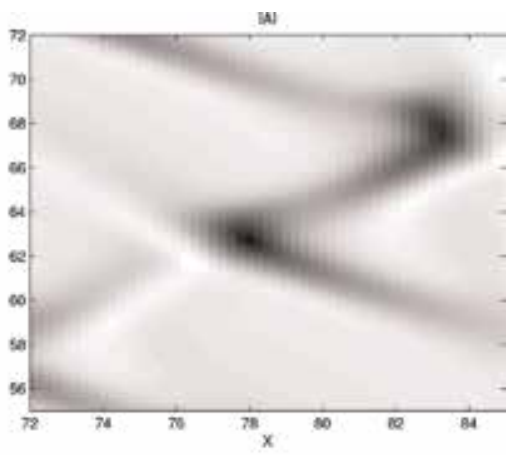

b)

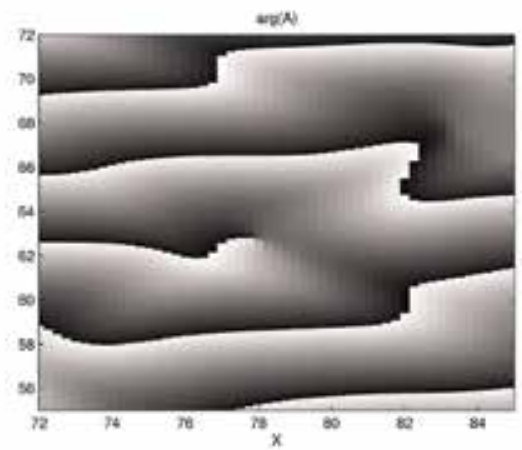

c)

Figure 3 .

Space-time gray-scale plots showing the invasion of a plane wave state by hole-defect chaos: (a) wave amplitude, $|A|($ dark: $|A| \approx 0)$, and $(b)$ close-up of $c_{3}=0.5 ; c_{5}=1.1$ showing in detail how a hole generates a phase defect that, in turn, generates two daughter holes: $c_{1}=0.59, c_{3}=0.5, c_{5}=1.1$.

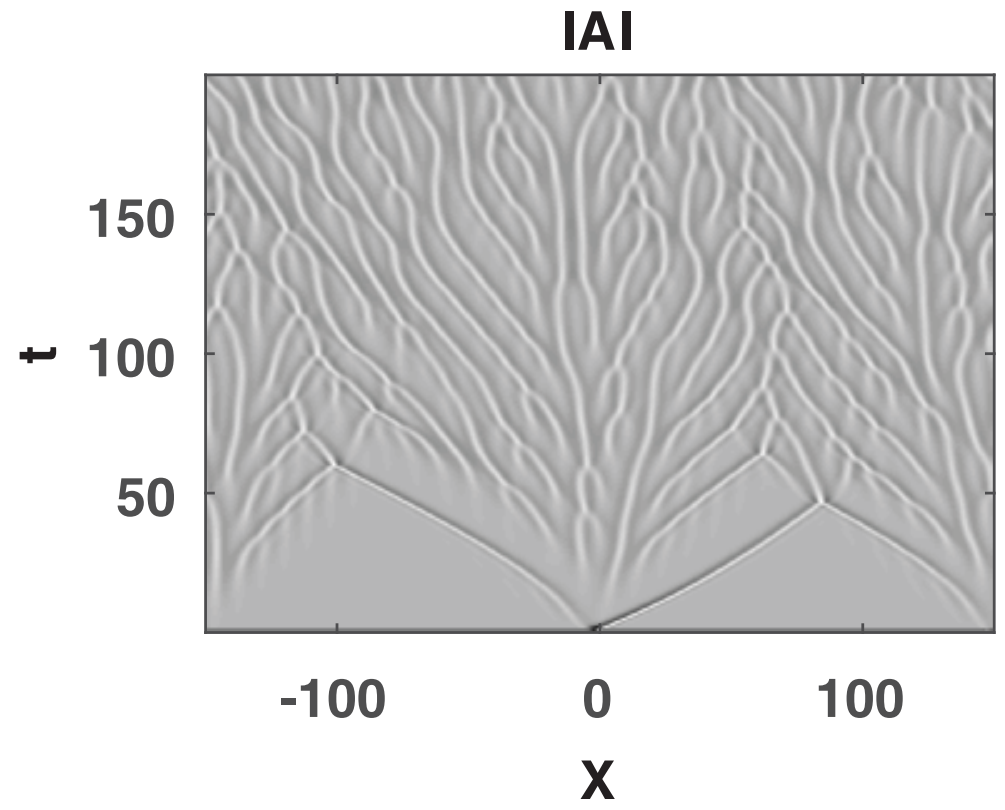

Figure 4 .

Phase turbulence regime of $|A|$, for $c_{1}=1.5, c_{3}=0.5$, and $c_{5}=0.5$. 
wavenumbers, reminiscent of the Kuramoto-Sivashinsky equation (KS). The dynamics is in fact very similar to that of KS, which is not surprising since this equation was originally derived to describe the phase dynamics of CGL equation near the BFN line.

\subsubsection{Weak turbulence regime}

Beyond the BFN line, we observe that for the parameter equations $\left(c_{1}, c_{5}\right)$ larger, a weak turbulence regime is observed [2] (see Figure 5). Weak turbulence theory was developed in the 1960 s to provide equations which quantitatively describe the transfer of energy among turbulent, weakly nonlinear, and dispersive waves in fluids [34]. The basic or kinetic equations produced by weak turbulence theory have been applied to analyze energy transfer including internal and surface waves with small aspect ratios in the atmosphere and ocean [35]. They are also observed in the case of two spatial dimensions [3, 36]. As we observe in Figure 5, holes move across the system (darker lines in Figure 5), while the amplitude of wave patterns at their cores changes. A black region along a gray line indicates amplitude $|A|$ near zero at the core of the hole. Each hole may spawn new holes, which in turn contribute to the loss of spatial coherence of the solution. Figure 6 shows the evolution of one hole of Figure 5 from its creation to its disappearance.

\subsubsection{Defect turbulence}

Father away from the BFN line a spatiotemporally disordered regime called amplitude or defect turbulence is observed (see Figure 7). The behavior in this region is characterized by defects. The defect turbulence regime is the dynamical regime wherein the fluctuations of $|A|$ become dominant over the phase dynamics.

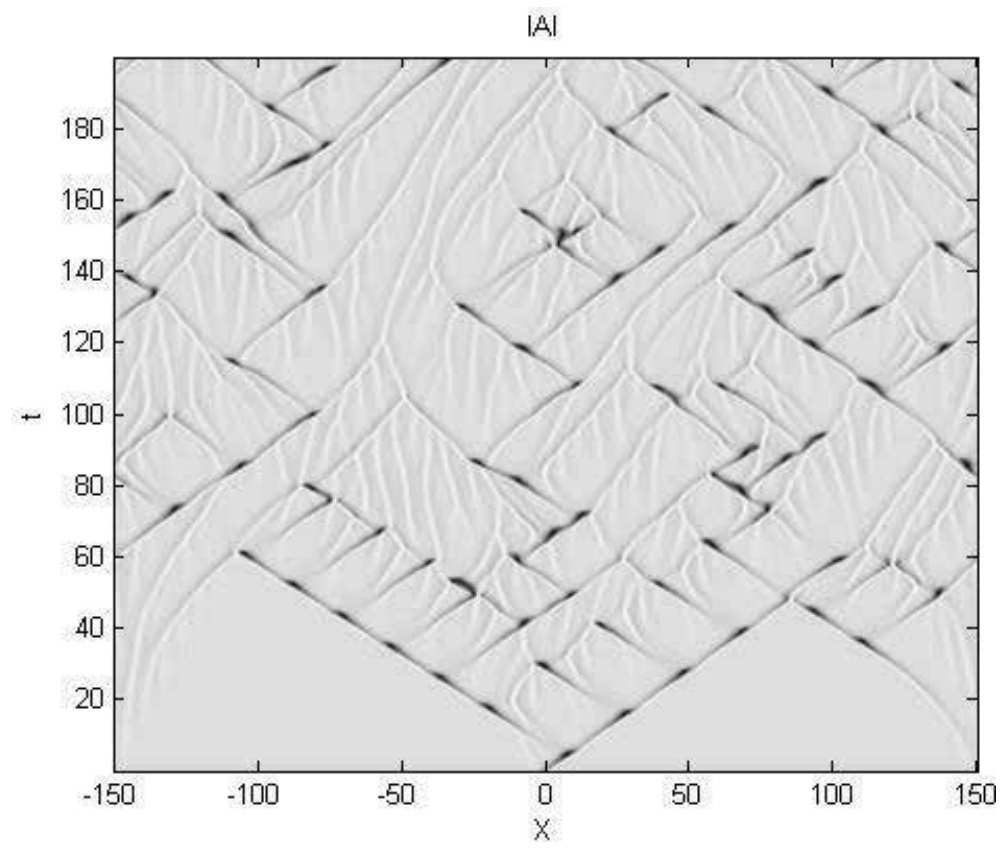

Figure 5 .

Weak turbulence regime observed for $c_{1}=1.5, c_{3}=0.5$, and $c_{5}=0.9$. 
Nonlinear Dynamical Regimes and Control of Turbulence through the Complex... DOI: http://dx.doi.org/10.5772/intechopen.88053

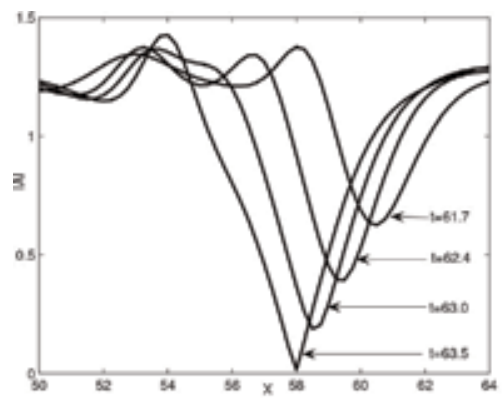

a)

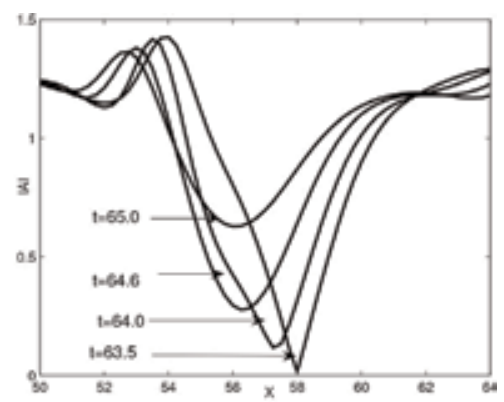

b)

Figure 6.

(a) Formation and $(b)$ disappearance of a hole between $t=61.5$ and $t=64.5$ in the numerical simulation of Figure 5.

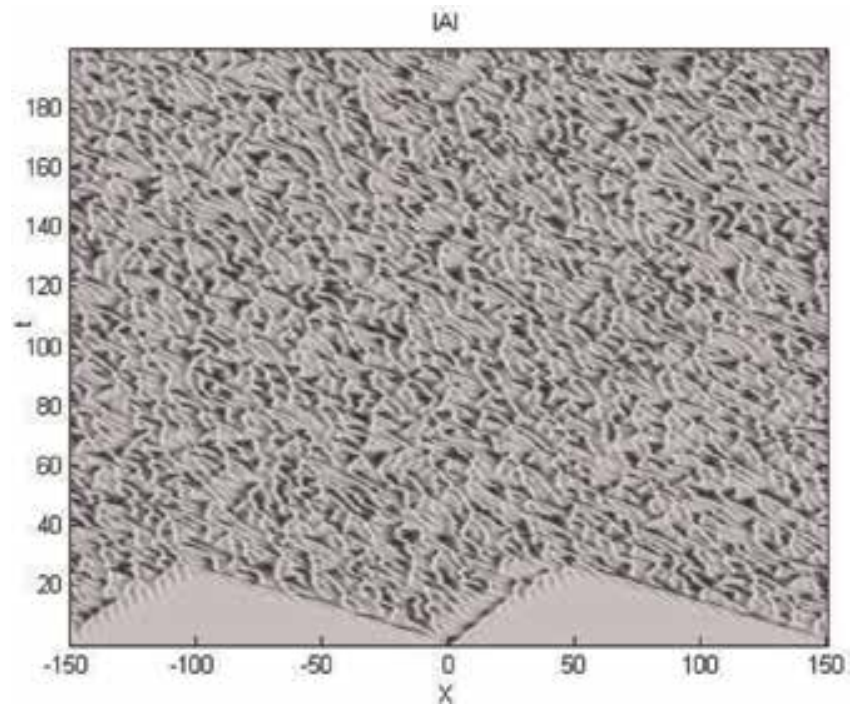

Figure 7.

Defect turbulence regime observed for $|A|$, with $c_{1}=2.5, c_{3}=0.5, c_{5}=1.1$.

The complex field experiences therefore large amplitude oscillations which can (locally and occasionally) cause $|A|$ to vanish. As a consequence, at all those points (hereinafter called space-time defects or phase singularities), the global phase of the field $\Phi \equiv \arctan \left[\frac{\operatorname{Im}(A)}{\operatorname{Re}(A)}\right]$ shows a singularity.

\section{Nonlinear structures of traveling waves in subcritical systems with finite geometries}

\subsection{The cubic-quintic complex Ginzburg-Landau equation in a finite domain}

The one-dimensional cubic-quintic CGL equation in this case is given by:

$$
\frac{\partial A}{\partial t}=\nu \frac{\partial A}{\partial x}+\left(1+i c_{1}\right) \frac{\partial^{2} A}{\partial x^{2}}+\mu A+\left(1-i c_{3}\right)|A|^{2} A-\left(1-i c_{5}\right)|A|^{4} A, \quad 0 \leq x \leq L .
$$


This equation describes the envelope of a traveling wave propagating at the group velocity $v$ toward negative $x$ [18]. $L$ is the length of the domain. This model equation arises in physics as an amplitude equation, providing a reduced universal description of weak nonlinear spatiotemporal phenomena in extended continuous media in the proximity of a subcritical Hopf bifurcation. The homogeneous boundary conditions are given by:

$$
A(x=0, t)=A(x=L, t)=0 .
$$

Figure 8 illustrates the deterministic evolution of wave pattern amplitude for convective instability and absolute instability regimes. In the case of convective regime, the wave patterns disappear with the time, while in the case of the absolute instability, they propagate in the whole system.

\subsection{Stability of wave patterns of the $1 D$ cubic-quintic CGLE}

Let us note that, in the convective regime, the localized disturbances of the basic state are growing but step away from the source. This is why we have restricted the study to the dynamics of pattern for parameters corresponding to the absolute instability regime. When the criticality parameter $\mu$ increases, the linear stability fails, and the waves can involve into new localized structures. The periodic basic solution loses its stability, a secondary instability appears, and we can observe new states in the domain (see Figure 9) $[18,37]$. As we can see in Figure 9, for a value the control parameter $\mu$ greater than a critical value $\mu_{c}$, secondary structures will appear at the left boundary of the system and destabilize the system $[4,18]$. These secondary structures create spatiotemporal chaos regime (regimes with defect, holes, etc.) into the system.

\subsection{Numerical simulations of the 1D cubic-quintic CGLE}

We investigate the effects of the quintic nonlinear dispersion coefficient $c_{5}$ in Eq. (5) with the homogeneous boundary conditions. The simulation was started

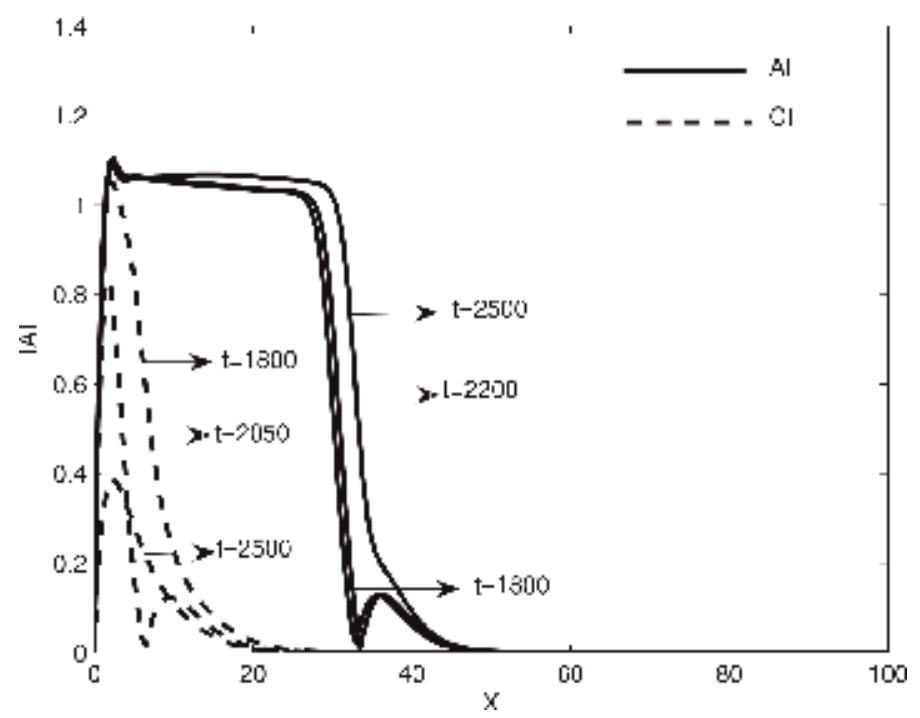

Figure 8.

Profiles of the amplitude of an initial perturbation at different times for $v=1.0, c_{1}=0.45, c_{3}=2.0, c_{5}=2.0$, and $L=100$ : in the convective instability (CI) regime with $\mu=0.15$ (dashed lines) and in the absolute instability (AI) regime with $\mu=0.3$ (solid lines). 
Nonlinear Dynamical Regimes and Control of Turbulence through the Complex...

DOI: http://dx.doi.org/10.5772/intechopen.88053

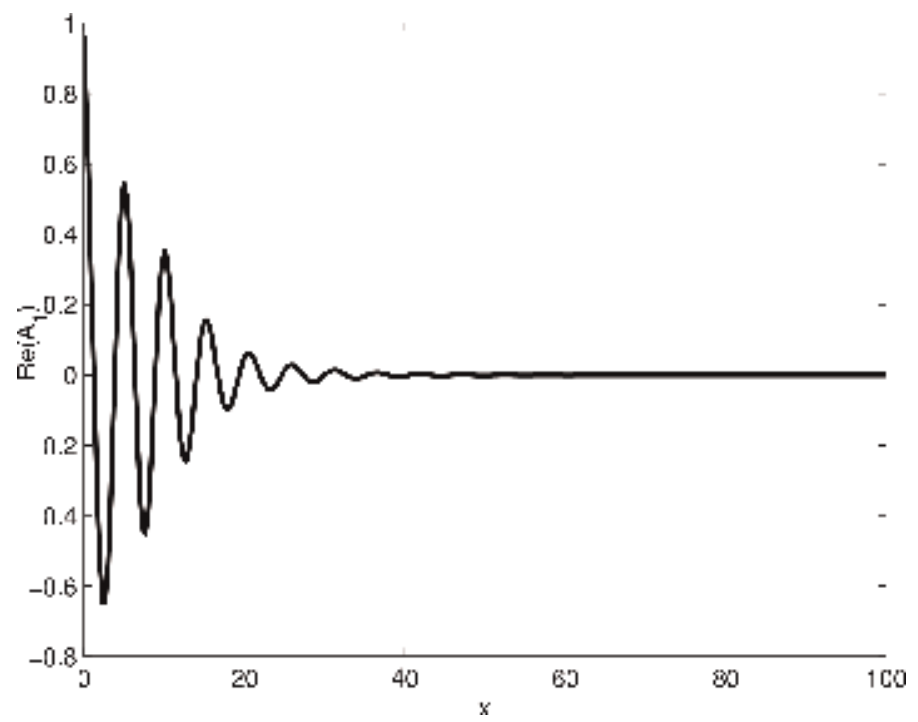

Figure 9.

Graph of $\operatorname{Re}\left(A_{1}\right)$ for $c_{1}=0.45, c_{3}=2.0, c_{5}=2.0, L=100, \mu=1.4, \Omega=1.2$, and $v=1.0$.

from an approximation of a pulse-like solution with a low amplitude. We investigate the effects of the quintic nonlinear coefficient $c_{5}$ with the homogeneous Dirichlet boundary conditions. Solving the 1D cubic-quintic CGLE for several values of $c_{5}$ in the absolute instability regime leads to bifurcation of the global mode to new states that are summarized in the phase diagram of Figure $\mathbf{1 0}$ for $c_{5}$ and $\varepsilon$ [-1.5;3.5]. We have found that the threshold of convective-absolute instability is $\mu_{\alpha}=0.207$. Our phase diagram is obtained only in the absolute instability regime $\left(\mu>\mu_{\alpha}\right)$. For the positive values of $c_{5}$, global mode regime and chaotic regimes are observed; they are separated by the line $L_{1}$. The global mode is a stable regime. The secondary structures are created by the secondary front which is close to the

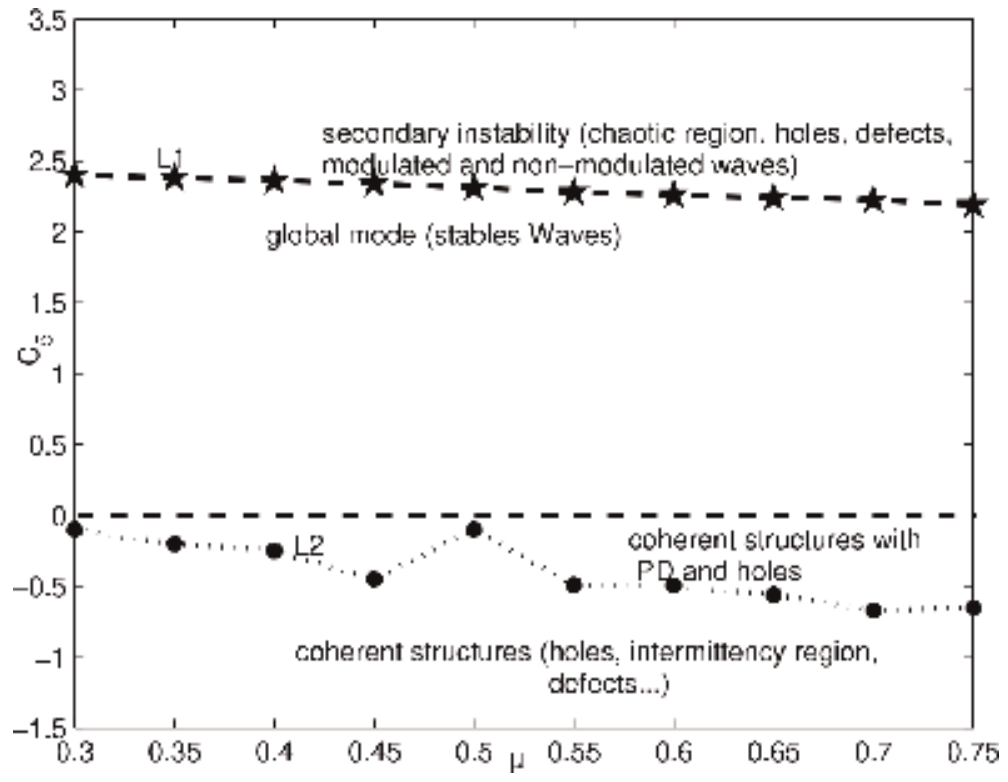

Figure 10.

Phase diagram in $\left(\mu, c_{5}\right)$, with $c_{1}=0.45, c_{3}=2.0, c_{5}=2.0, L=100$, and $v=1.0 . P D$ and $P H$ stand for periodic defects and periodic hole regions, respectively. 

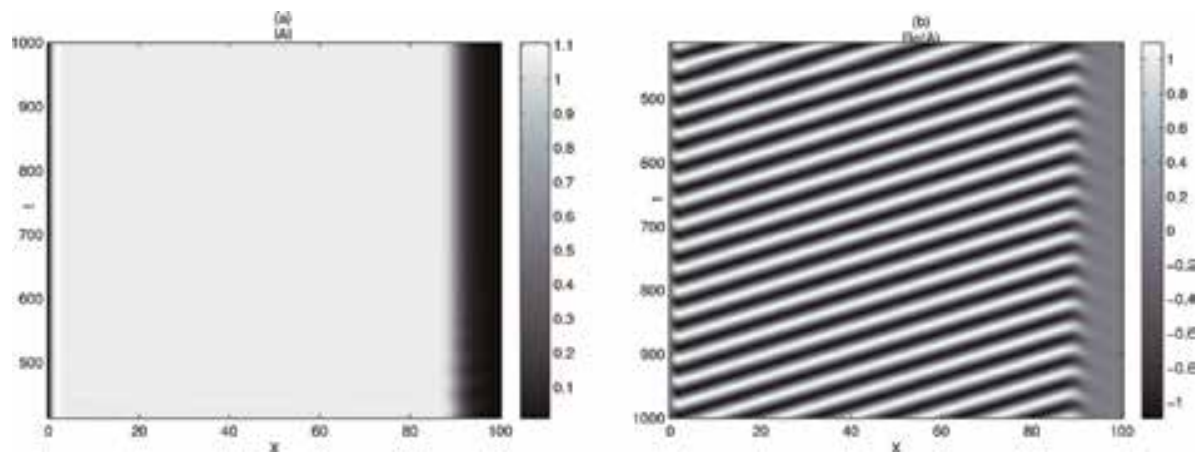

Figure 11.

Space-time variation of the $\operatorname{Re}(A)$ for $\mu=0.3, c_{1}=0.450, c_{3}=2.0, c_{5}=2.0, L=100$ and $\nu=1$, showing global modes.

downstream boundary. The global mode regime is represented by the phase plots in Figure 11. This figure demonstrates the selection of the stable waves in the global mode. It reveals that the wave patterns are decreasing in time and propagate in only one direction toward the left. The variation of the coefficient $c_{5}$ leads to bifurcation of the global mode to new states which is observed in Figure 12. It reveals that the solution can break up into several disjoint states with different properties separated by more fronts defined by the location of the states. The regular pattern is destabilized and gives rise to spatiotemporal turbulent state near the wall $x=0$ (space $x_{1}$ ), which is followed by a modulated state in space and time (space $x_{2}$ ), while, near the end $x=\mathrm{L}$, the wave pattern remains non-modulated, and the regular wave train is observed (space $x_{3}$ ) $[18,37]$. Defect and holes can be detected in the chaotic space. Defects and holes are local structures that play a crucial role in the intermediate regime between laminar states and hard chaos. Defects are points in the space-time diagram where the amplitude of the wave vanishes and the phase is not defined. In two and higher dimensions, such defects can disappear only via collisions with other defects and act as long-living seeds for local structures like spirals [38]. We have noticed that when $c_{5}$ further increases, the chaotic region

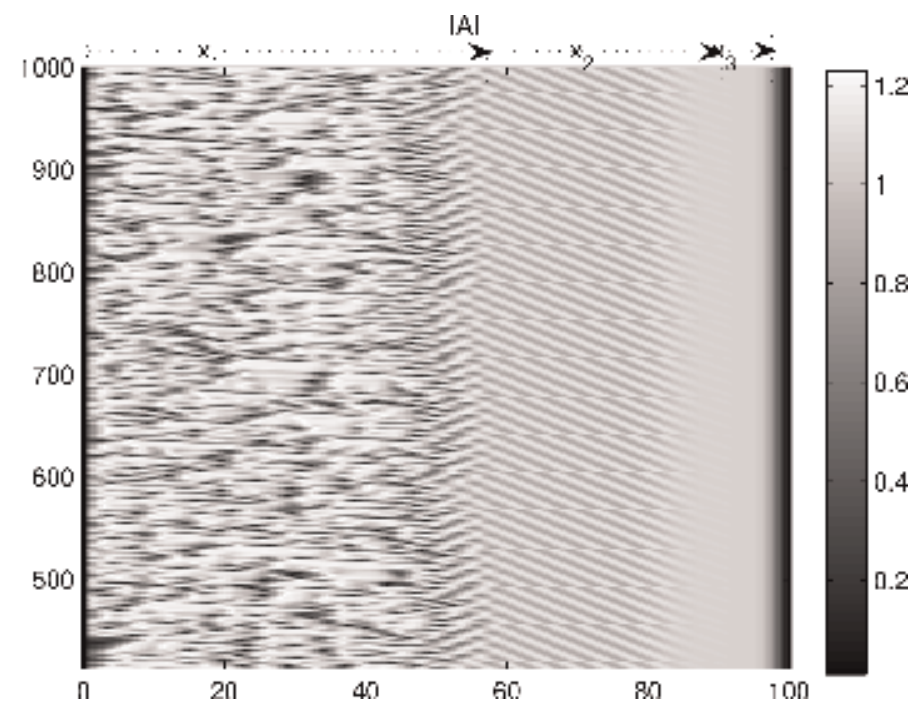

Figure 12.

Space-time variation of the wave pattern amplitude $|A|$, for $\mu=0.65, c_{1}=0.450, c_{3}=2.0, c_{5}=2.37$, $L=100$, and $\nu=1$, indicating the appearance of the secondary structures. 


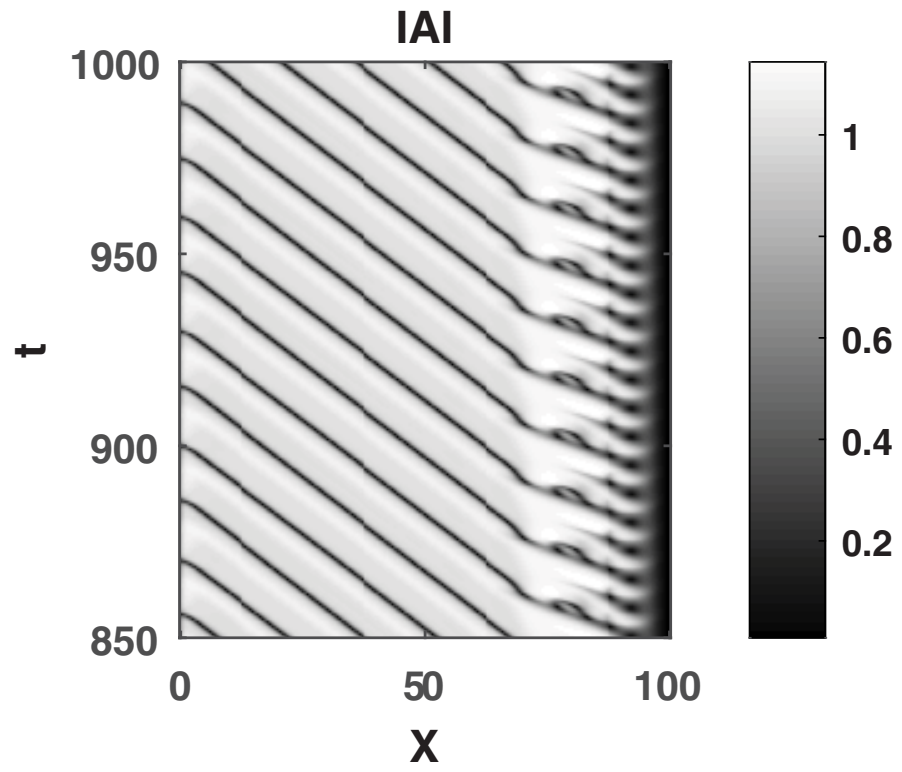

Figure 13.

Space-time variation of the wave amplitude $|A|$, for $\mu=0.35, c_{1}=0.450, c_{3}=2.0, c_{5}=? 0.2, L=100$, and $\nu=1$, denoting coherence structure with $P D$ and $P H$.

propagates into the domain toward the upstream boundary and the system becomes more and more chaotic. For negative values of $c_{5}$, the regimes observed are different. The domain exhibits global modes, amplitude defects, holes, and spatiotemporal intermittency. Figure 13 reveals that the wave patterns are stable at the first part of the domain until the three quarter of the domain. Near the upstream boundary, the news structures are revealed (hole, defects, etc.). The defects appear at regular time intervals around $x=80$, and for this reason we call them time-periodic defects (PD). The periodic topological defects have been observed, for example, in many experiences like the miscible fluid convection [39] and the system of Taylor-Dean [40]. Near the right boundary, holes are observed and also before the PD, around

(bị

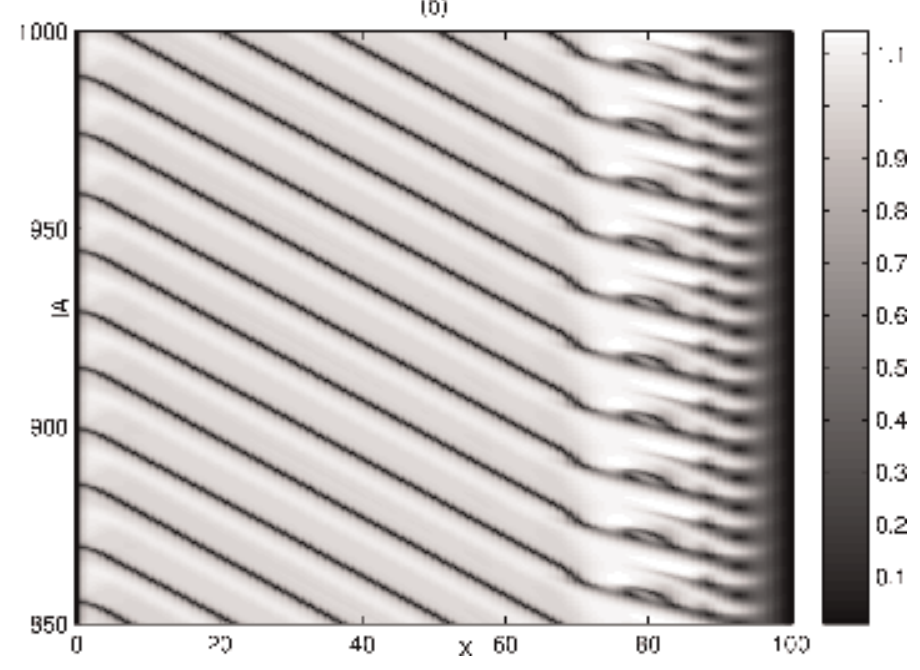

Figure 14.

Space-time variation of the wave pattern amplitude $|A|$ showing also coherence structures with $P D$ and $P H$, for $\mu=0.3, c_{1}=0.450, c_{3}=2.0, c_{5}=? 0.25, L=100$, and $\nu=1$. 


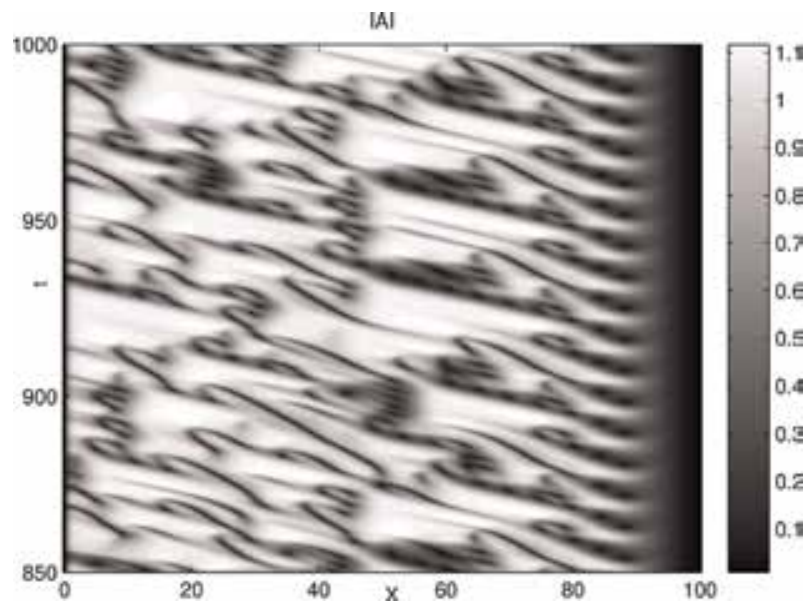

Figure 15.

Space-time variation of the wave pattern amplitude $|A|$, for $\mu=0.3, c_{1}=0.450, c_{3}=2.0, c_{5}=? 0.6$, $L=100$, and $\nu=1$, displaying spatiotemporal intermittency, defects, and holes.

$x=70$. Holes which appear near the upstream boundary are regular in time interval, and we call them periodic holes (PH). Figure 14 is obtained for another value of $c_{5}$. It shows a periodic sequence of phase jumps propagating in the advection direction, i.e., to the left; the wave pattern amplitude is modulated in this region. We notice that periodic defects and periodic holes are advected toward the upstream boundary. For the increasing values of $c_{5}$, the space-time of Figure 15 reveals that the system becomes a more disordered regime and more complex. This space-time reveals the disordered regimes in the domain and the behavior of the patterns become more complex. The figure reveals the presence of the core of defects around $x=85$ which are periodic. Before the periodic defects, a disordered regime is observed. This disordered regime is a spatiotemporal intermittency regime.

\section{Controlling spatiotemporal chaos in one-dimensional systems near subcritical bifurcation}

\subsection{The dynamical model}

The modified cubic-quintic CGLE is given by [20]:

$$
\frac{\partial A}{\partial t}=\left(1+i c_{1}+\lambda\left(\frac{|A|^{2}}{\left|A_{0}\right|^{2}}-1\right)\right) \frac{\partial^{2} A}{\partial x^{2}}+\mu A+\left(1-i c_{3}\right)|A|^{2} A-\left(1-i c_{5}\right)|A|^{4} A,
$$

where $\lambda=\lambda_{r}+i \lambda_{i}$ is a complex constant. Notice that the term $\lambda\left(\frac{|A|^{2}}{\left|A_{0}\right|^{2}}-1\right)$ vanishes identically for $A=A_{0}$. The added term also preserves the phase invariance of the solution of the original cubic-quintic CGLE, $A \rightarrow A e^{i \phi}$, with $\phi$ being an arbitrary phase.

\subsection{Numerical simulation}

We start by assuming that the system is in a deeply chaotic region, i.e., parameters are chosen from defect turbulence area in order to verify the results obtained from the linear stability analysis. Figure $\mathbf{1 6}$ plots the trajectory in phase space, in 
Nonlinear Dynamical Regimes and Control of Turbulence through the Complex... DOI: http://dx.doi.org/10.5772/intechopen.88053

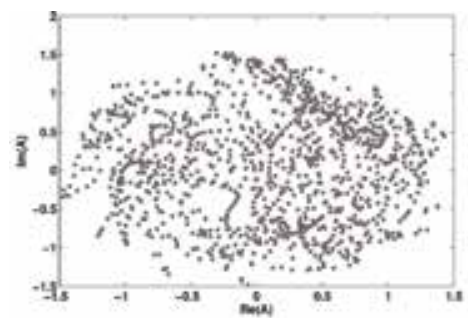

a)

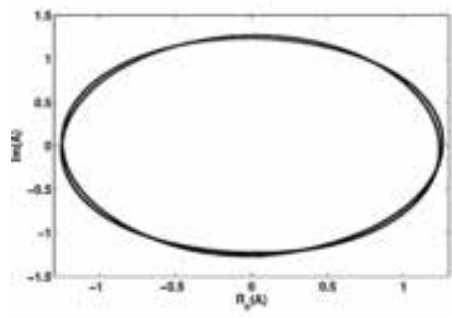

c)

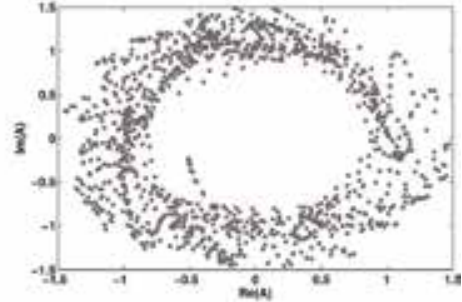

b)

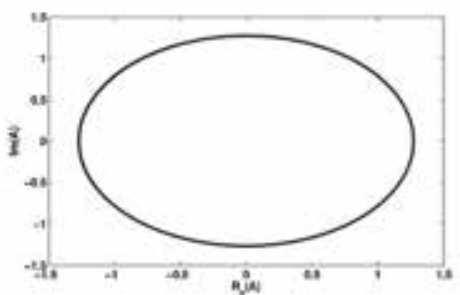

d)

Figure 16.

Plot of $\left(k, c_{1}\right)$ for $c_{3}=0.5, c_{5}=1.1$ with (a) $\lambda_{r}=0.0$, et $\lambda_{i}=0.0,(b) \lambda_{r}=1.0$, et $\lambda_{i}=0.0,(c) \lambda_{r}=2.5$, et $\lambda_{i}=1.1,(d) \lambda_{r}=3.0$, et $\lambda_{i}=1.5$, with $\sigma=0.008, k=0.85$, the dashed lines correspond to the BFN line of the original cubic-quintic CGLE, the solid lines correspond to the BFN line of the modified cubic-quintic CGLE. The shadowed region corresponds to the stability region.

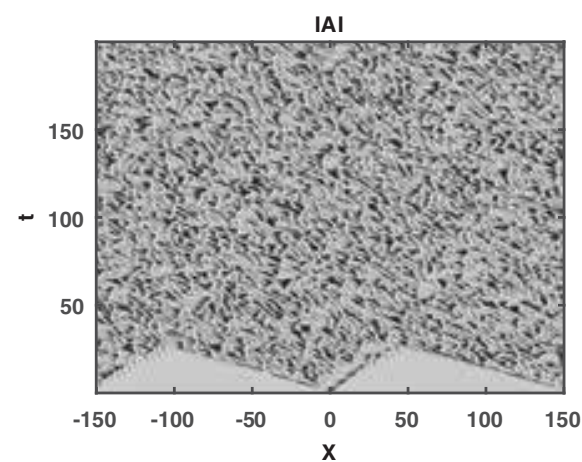

(a)

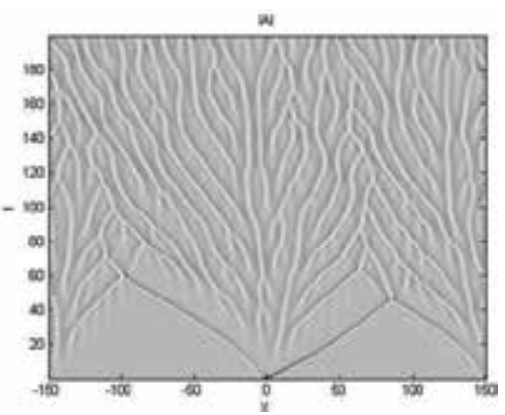

(b)

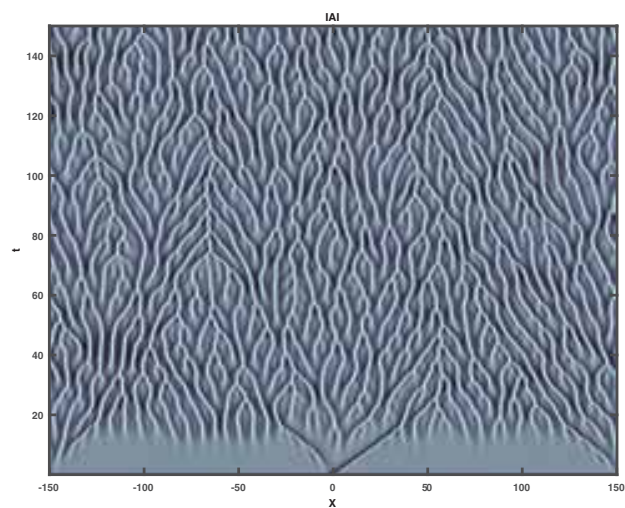

(c)

Figure 17.

Transition from chaotic behavior to quasi-periodic state for $c_{1}=2.5, c_{3}=0.5, c_{5}=1.1$ : When $(a) \lambda_{r}=0$ and $\lambda_{i}=o$ (chaos with defects); (b) $\lambda_{r}=2.0$ and $\lambda_{i}=0.5$ (phase turbulence); (c) (plane wave) $\lambda_{r}=3.2$ and $\lambda_{i}=1.8$, with $\sigma=0.008, k=0.85$, and $N=1200$ grid points, leading to the plane wave regime. 
which the system parameters are exemplified as $c_{1}=2.5, c_{3}=0.5$, and $c_{5}=1.1$ [15]. The chaotic motion is obvious: the amplitude of variable incidentally drops to zero to produce defects (see Figure 16a). Now we control spatiotemporal chaos by performing nonlinear diffusion parameters. As an example, we let $\lambda_{r}=2.0$ and $\lambda_{r}=0.5$; the trajectory of system is illustrated in Figure 16b. The real part of system variable is confined into a finite range, and defect turbulence is no longer being observed, whereas the chaotic motion is not eliminated completely. For other values of the diffusion parameter as $\lambda_{r}=3.0$ and $\lambda_{r}=1.5$, for example, the chaotic motion is suppressed, and period-doubled states are observed as is illustrated in Figure 16c. The solution has double periodicity since double loop is observed. For the value of $\lambda$ being more larger, the chaotic motion is still suppressed, but the periodic states are observed. The system is more stable. The double loop is merged into a single loop, as is shown in Figure 16d. To get also an idea of how the spatiotemporal dynamics of the system changes in the parameter range of $|\lambda|$ between turbulence and uniform oscillations, we show in Figure 17a a series of space-time plots of asymptotic dynamical states reached for different values of $|\lambda|$. For the increasing values of $|\lambda|$, the transition from defect turbulence to plane wave regime is observed

(Figure 17a-c).

\section{Time-delay auto-synchronization control of defect turbulence in cubic-quintic complex Ginzburg-Landau equation}

\subsection{Model equation}

To control the different turbulence regimes observed in the domain, a global feedback term can be introduced. The modified cubic-quintic CGL eqiuation is given by $[27,41,42]$.

$$
\begin{gathered}
\frac{\partial A}{\partial t}=\left(1+i c_{1}\right) \frac{\partial^{2} A}{\partial x^{2}}+\mu A+\left(1-i c_{3}\right)|A|^{2} A-\left(1-i c_{5}\right)|A|^{4} A+F, \\
F(t)=\alpha e^{i\left(\chi_{0}+\omega \tau\right)} \bar{A}(t-\tau),
\end{gathered}
$$

where

$$
\bar{A}(t)=\frac{1}{L} \int_{0}^{L} A(x, t) d x
$$

denotes the spatial average of $A$ over a one-dimensional medium of length $L$.

The parameter $\alpha$ describes the feedback strength, and $\chi_{0}$ characterizes a phase shift between the feedback and the dynamics. $\omega$ denotes the frequency of the oscillation and $\tau$ is the delay time. When delay time is short $(\tau<<1)$, the slowly varying average amplitude $\bar{A}(t)$ does not significantly change within the delay time, and the delays in this term could be neglected. We obtain then the following equation:

$$
\frac{\partial A}{\partial t}=\left(1+i c_{1}\right) \frac{\partial^{2} A}{\partial x^{2}}+\mu A+\left(1-i c_{3}\right)|A|^{2} A-\left(1-i c_{5}\right)|A|^{4} A+\alpha e^{i \chi} \bar{A}(t),
$$

where $\chi=\chi_{0}+\omega \tau$. Eq. (11) does not include any delay. Nonetheless, the delay time $\tau$ plays an important role here, because it determines the effective phase shift $\chi$ in the equation. The variation of $\tau$ provides a simple way for changing the phase shift of the global feedback and thus the feedback effects. 
Nonlinear Dynamical Regimes and Control of Turbulence through the Complex... DOI: http://dx.doi.org/10.5772/intechopen.88053

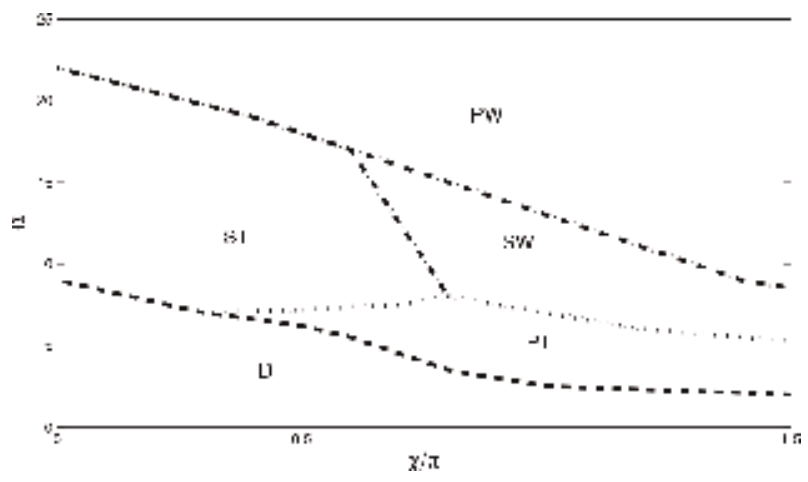

Figure 18.

Phase diagram of $(\chi, \alpha)$ showing different types of dynamical regimes: Defect turbulence $(D T)$, spatiotemporal intermittency (STI), phase turbulence $(P T)$, standing wave $(S W)$, and plane wave $(P W)$ for $c_{1}=2.5, c_{3}=$ $0.5, c_{5}=1.1$. Phase diagram of $(\chi, \alpha)$ showing different types of dynamical regimes: Defect turbulence $(D T)$, spatiotemporal intermittency (STI), phase turbulence (PT), standing wave (SW), and plane wave (PW) for $c_{1}=2.5, c_{3}=0.5, c_{5}=1.1, \mu=1.0, \mu=1.0$.

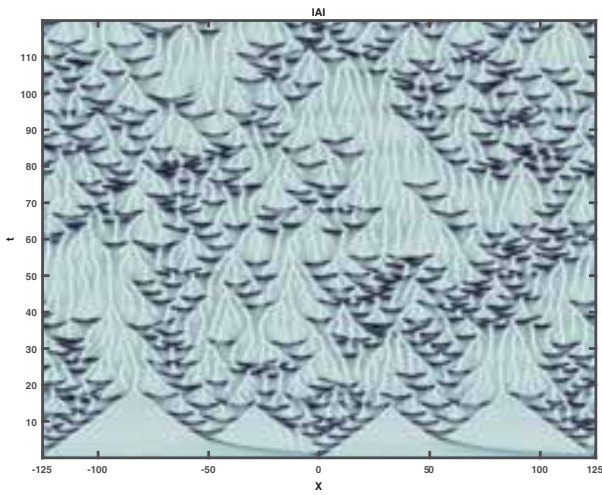

(a)

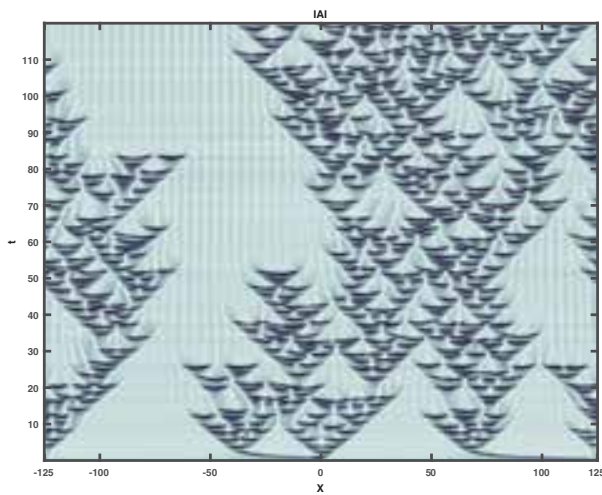

(a)

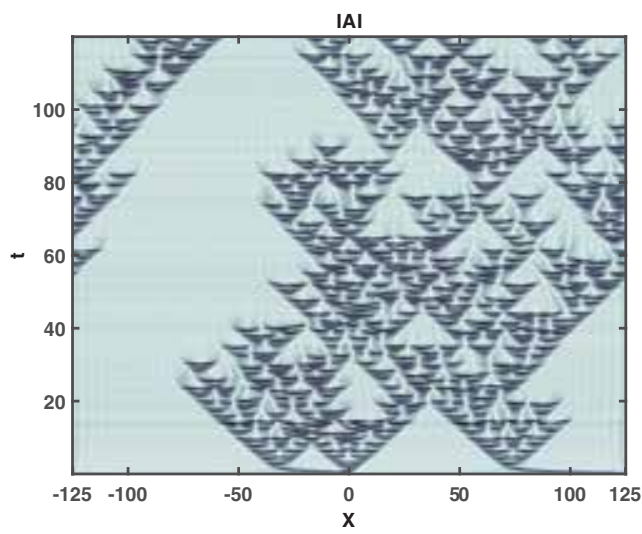

(c)

Figure 19.

Transition from defect turbulence to spatiotemporal intermittency regime for $c_{1}=2.5, c_{3}=0.5, c_{5}=1.1$, $\chi=\pi / 6$, and $\mu=1.0$, when (a) $\alpha=8.0$, (b) $\alpha=12.0$, (c) $\alpha=15$.

\subsection{Numerical simulation}

In this section, we present the results of a numerical study of Eq. (11). We analyze the stability of the unstable wave patterns observed inside a defect 
turbulence regime by using global feedback term [27]. We study the effects of the feedback term on the system. A sufficient strong feedback can suppress spatiotemporal chaos and establishes uniform oscillations. We show that for certain values of the global feedback term and the delay time, the system which initially was chaotic becomes completely stable. The dynamic regimes observed during the numerical study are summarized in the state diagram of Figure 18. The five regimes observed are defect turbulence, spatiotemporal intermittency, phase turbulence, standing waves, and plane waves. We remark that as the feedback intensity is increased starting from zero, global oscillations set in, and defect turbulent regimes are replaced by other interesting regimes until the appearance of the laminar state.

For certain values of $\alpha$, amplitude defects disappear from some parts of the system, and thus, an intermittent state is developed. Figure 19 illustrates three examples of intermittent regimes depending on the values of $\alpha$ and $\chi$ in a 1D system $[27,42]$. Figure 19a shows that turbulent bursts which occupy most of the system and laminar areas are relatively rare, while Figure 19b and c shows the coexistence between turbulence and laminar state filled with standing wave (see Figure 19b) or plane waves (see Figure 19c). By further increasing the feedback intensity from the states of intermittent turbulence, uniform oscillations are observed for the phase shift in the interval $0<\chi<3 \pi / 4$. For appropriate values of $\alpha$ and $\chi$, the domain is still always turbulent, but the defects disappeared completely. The wave pattern is

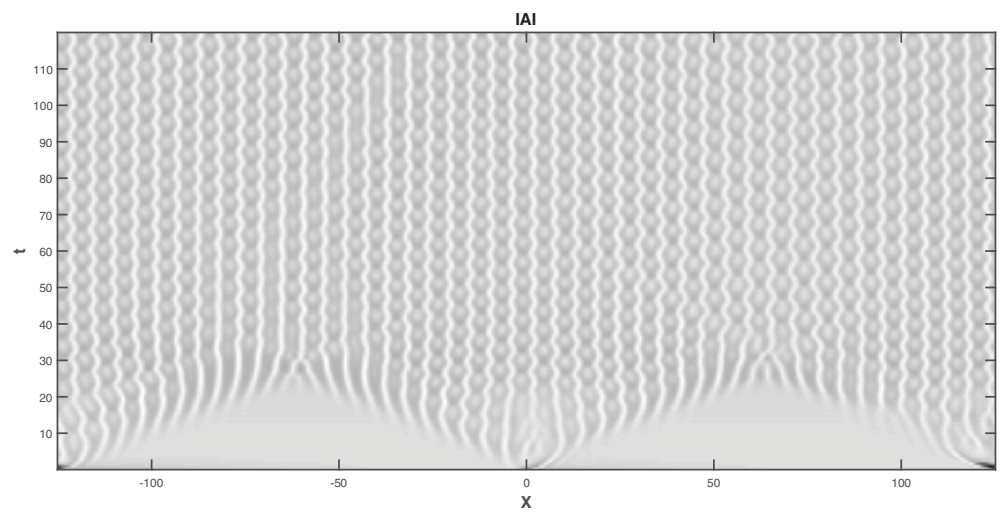

Figure 20.

Phase turbulence regime of wave pattern amplitude $|A|$ obtained for $\alpha=6.0, c_{1}=2.5, c_{3}=0.5, c_{5}=1.1$, $\chi=7 \pi / 4, \mu=1.0$.

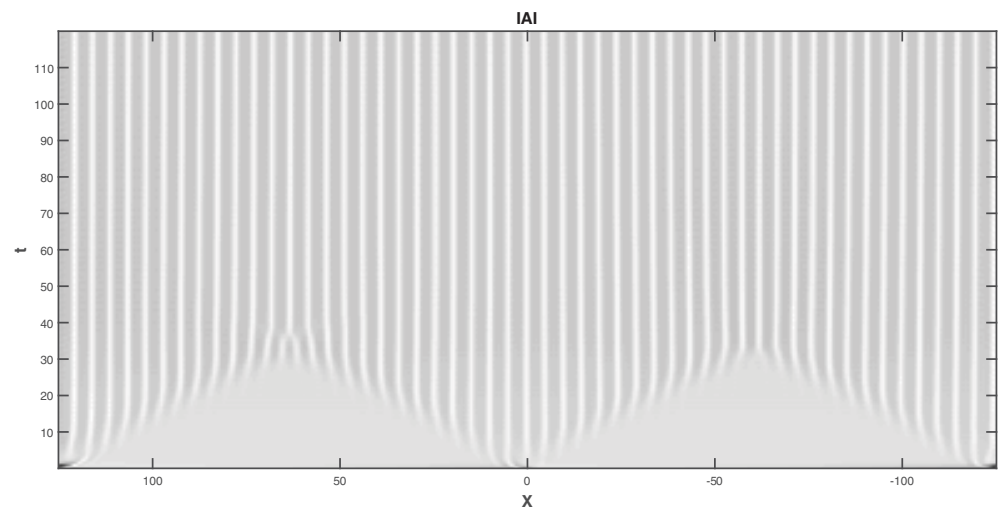

Figure 21.

Space-time variation of the wave pattern amplitude $|A|$ obtained for $\alpha=9.0, c_{1}=2.5, c_{3}=0.5, c_{5}=1.1$, $\chi=7 \pi / 4, \mu=1.0$. 
Nonlinear Dynamical Regimes and Control of Turbulence through the Complex...

DOI: http://dx.doi.org/10.5772/intechopen.88053

in the phase turbulence regime. It is illustrated in Figure 20. In this regime, the amplitude is always bounded away from zero. The amplitude $A$ never reaches zero and remains saturated. We notice that for certain values of $\alpha$ and $\chi$, standing waves, also called stationary wave, are observed (see Figure 21). Standing waves have been observed in the model of CO oxidation under intrinsic gas phase coupling [43,44].

\section{Discussion}

Let us now introduce to the CGL equation the global time-delay feedback and study its effects on the system. The new CGL equation with time-delay autosynchronization is given by:

$$
\frac{\partial A}{\partial t}=\left(1+i c_{1}\right) \frac{\partial^{2} A}{\partial x^{2}}+(\mu-i \omega) A+\left(1-i c_{3}\right)|A|^{2} A-\left(1-i c_{5}\right)|A|^{4} A+F,
$$

where $F$ is a feedback term given by

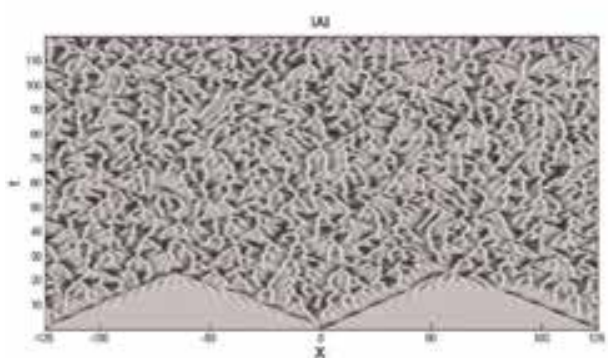

a

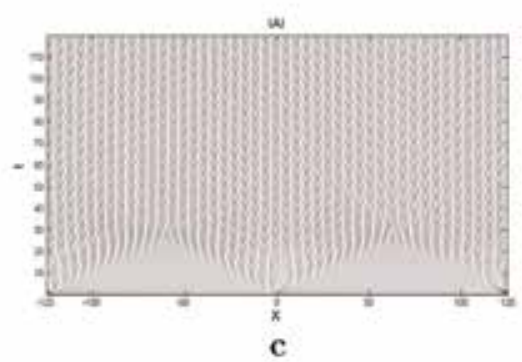

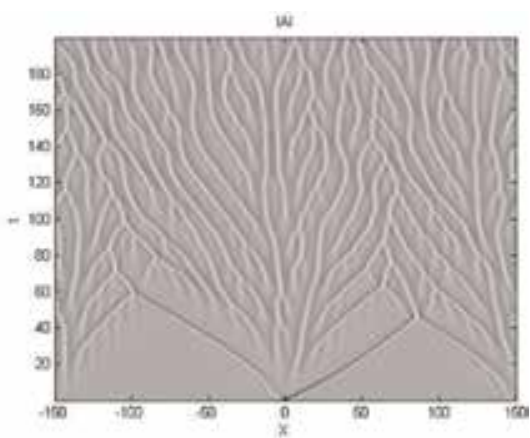

b

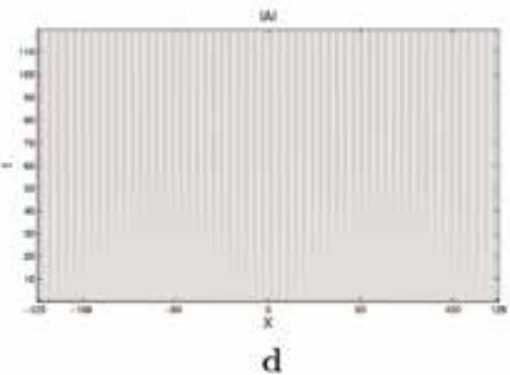

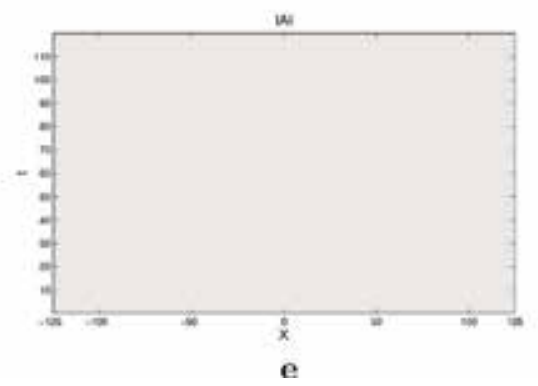

Figure 22.

Space-time variation of the wave pattern amplitude $|A|, \chi=\pi / 2, c_{1}=2.5, c_{3}=0.5, c_{5}=1.1, \mu=1.0$, and $\tau=1.2$ for $(a) \alpha=0.0$ showing $D T,(b) \alpha=12.0$ showing $W T,(c) \alpha=15$ showing $P T$, and $\alpha=15$ showing $P W$. 


$$
F(t)=\alpha e^{i \chi}(\bar{A}(t-\tau)-\bar{A}(t))
$$

and

$$
\bar{A}(t)=\frac{1}{L} \int_{0}^{L} A(x, t) d x,
$$

with the amplitude and frequency given by

$$
\left|A_{1}\right|^{2}=\frac{1}{2}(1 \pm \sqrt{1+4(\mu+\alpha[\cos (\chi-\Omega \tau)-\cos (\chi)])})
$$

and

$$
\Omega=-\omega-\left|A_{1}\right|^{2}\left(c_{3}-c_{5}\left|A_{1}\right|^{2}\right)+\alpha[\sin (\chi-\Omega \tau)-\sin (\chi)] .
$$

A cubic CGL equation with a similar feedback scheme has been investigated in Refs. $[13,35]$. In their work, they have shown how the strongly disordered state can be stabilized in the system. The initially unstable system undergoes several transformations successively and become stable; we have in order defect turbulence, phase turbulence, standing wave state, and uniform oscillations. The results of our numerical study of Eq. (12) are given in Figure 22. This figure shows the progressive transition from defect turbulence to plane wave state. In the absence of feedback (see Figure 22a, with $\alpha=0$ ), defect turbulence is observed. For small $\alpha$, weak turbulence is observed (see Figure 22b). When the feedback term grows, the system displays a disordered state of phase turbulence (see Figure 22c), stationary standing wave patterns (see Figure 22d), and uniform oscillations (see Figure 22e), respectively.

\section{Effects of nonlinear gradient terms on the defect turbulence regime in weakly dissipative systems}

The LOCGL equation which describes a system exhibiting a subcritical bifurcation to traveling waves must contain a quintic nonlinearity; at this order, it is necessary to include the lower-order nonlinear gradient terms:

$$
\begin{array}{r}
A_{t}+V A_{x}=\left(\chi_{r}+i \chi_{i}\right) A+\left(1+i c_{1}\right) A_{x x}+\left(1-i c_{3}\right)|A|^{2} A- \\
\left(1-i c_{5}\right)|A|^{4} A-q_{1}|A|^{2} A_{x}-q_{2}|A|_{x}^{2} A-q_{3} A^{2} A_{x}^{*},
\end{array}
$$

with $q_{1}=q_{1 r}+i q_{1 i}, q_{2}=q_{2 r}+i q_{2 i}$, and $q_{3}=q_{3 r}+i q_{3 i}$. Here, $A(x, t)$ describes the amplitude of extended spatial patterns. The values $\mathrm{q}_{1}, \mathrm{q}_{2}$, and $\mathrm{q}_{3}$ represent coefficients of nonlinear gradient terms. Two of these nonlinear gradient terms, i.e., $|A|^{2} A_{x}$ and $A^{2} A_{x}^{*}$, appear naturally in the asymptotic derivation [30, 31, 45-47]. Since we take the periodic boundary conditions, the convective term $V A_{x}$ may be transformed away by going into a moving frame of reference. Also, the parameter $\chi=\chi_{r}+i \chi_{i}$, which is proportional to the distance from criticality, can be taken as real, since the imaginary part can be transformed away by a simple transformation.

The aim is to see the impact of these nonlinear gradient terms on a defect turbulence regime. We use the indicators such as the Lyapunov exponent and the energy bifurcation diagram to confirm the nature of the regime. 


\subsection{Numerical simulations}

\subsubsection{Dynamical indicators}

We will essentially characterize the different types of dynamical behavior of the system by the energy function $Q$ and the largest Lyapunov exponent $\lambda_{\max }$. The first one is defined by

$$
Q(t)=\frac{1}{2 L} \int_{-L}^{L}|A(x, t)|^{2} d x
$$

which is frequently used to characterize non-regular dynamics in optics [48], localized patterns in fluids, and other physical systems, respectively [49]. The onedimensional system is assumed to be of length $2 L$. In order to check more dynamical behaviors of the system and provide a more quantitative aspect of the dynamics, we calculate the largest Lyapunov exponent defined by [50,51].

$$
\lambda_{\max }=\lim _{t \rightarrow \infty}\left[\frac{1}{t} \ln \left(\frac{\||\delta A(x, t) \||}{\||\delta A(x, 0) \||}\right)\right], \quad \text { with } \quad\|\delta A(x, t)\|=\left\{\int_{x=-L}^{x=L}|\delta A(x, t)|^{2} d x\right\}^{1 / 2},
$$

where $\delta A$ is a small perturbation such as $A=A_{0}+\delta A$ and $A_{0}$ is the initial value of the amplitude wave. Here, $\delta A(x, 0)=10^{-4} A_{0}$, and $\delta A$ satisfies the linearized evolution equation

$$
\frac{\partial \delta A}{\partial t}=\overline{\mathbf{J}} \cdot \delta A,
$$

where $\overline{\mathbf{J}}$ is the Jacobian matrix. The largest Lyapunov exponent is the dynamical invariant most easily and accurately estimated from experimental time series. This method has been extensively used for many different dynamical systems to quantify chaos [30]. The value $\lambda_{\max }$ can be positive or negative. Complex behaviors such as chaos and spatiotemporal chaos are confirmed by positive $\lambda_{\max }$. On the other hand, periodic or quasi-periodic solutions and non-chaotic attractors are characterized by negative $\lambda_{\max }$.

\subsubsection{Numerical results}

We will present here some of data obtained for systems with the presence of the nonlinear gradient terms. The results are summarized in Figures 23-28. In particular, we study the influence of the nonlinear gradient terms in the defect turbulence regime. Figure 23 shows the wave patterns and the energy as a function of time corresponding to the laminar regime. We notice that the system that was initially chaotic becomes completely stable by the presence of the nonlinear gradient terms. The chaos has been eliminated. By changing the values of nonlinear gradient terms, the dynamics of the system also change; it is confirmed by Figures 24 and 25 which represent the oscillating patterns. The corresponding largest Lyapunov exponent is zero.

The plot observed in Figure $\mathbf{2 4}$ is the running waves. They are quasi-periodic states; they move in one direction with constant speed, according to its initial condition; and this is the so-called oriental symmetry breaking. A double periodicity in time and in space is observed. In Figure 25, we have another type of the 


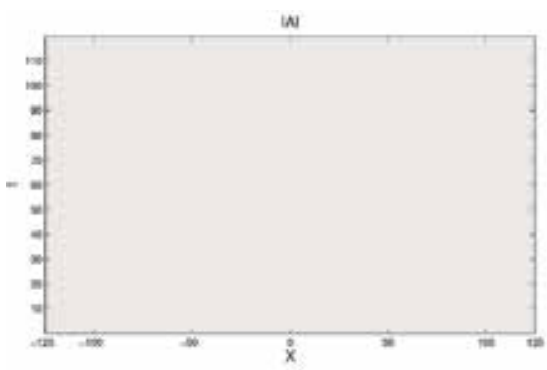

a)

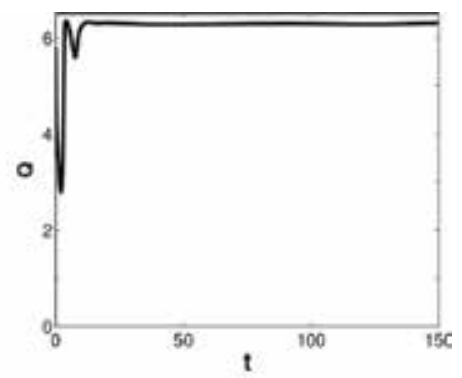

b)

Figure 23.

(a) Laminar regime and (b) pattern energy $Q$ as function of time for $q_{1}=1.5+0.5 i, q_{2}=q_{3}=1.5+1.5 i$.

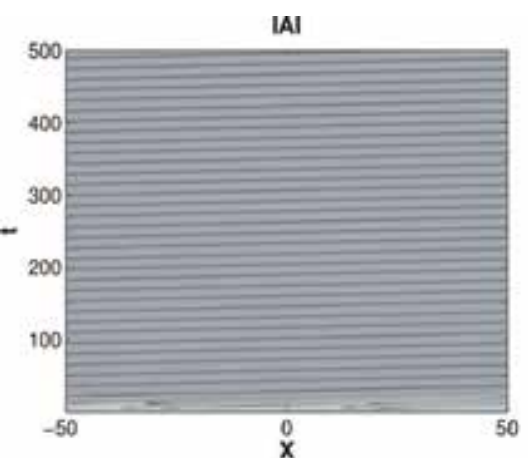

a)

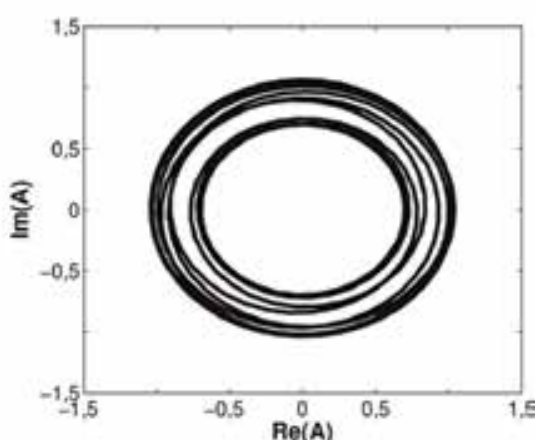

b)

Figure 24.

(a) Running wave regime, for $\chi=0.6 q_{1}=0.5+0.9 i, q_{2}=0.6+0.6 i, q_{3}=0.9+0.9 i$, and (b) phase portrait of running waves.

oscillating patterns in a color-coded space-time plot (see Figure 25a). After a transient time, the waves propagate uniformly, with a well-defined wave number and constant amplitude. We note also the presence of an attractor into the system which annihilate the wave patterns (see Figure 25b). The drop observed near $x=0$ expresses the fact that the initial condition is a hole. Figures 26-28 show the largest Lyapunov exponent $\lambda_{\max }$ and the bifurcation diagram of the pattern state as a function of the control parameter $\chi_{r}$ for Eq. (17). They allow us to see clearly how the

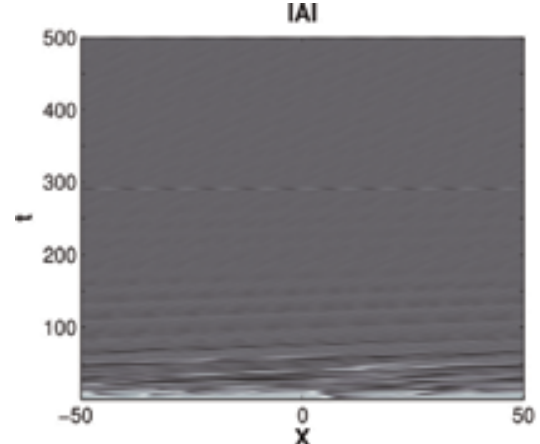

a)

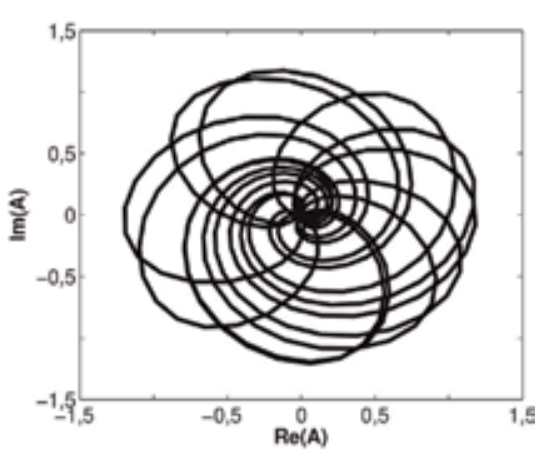

b)

Figure 25.

Oscillating regime in system, for $\chi=0.6 q_{1}=1.5+0.5 i, q_{2}=0.6+0.6 i, q_{3}=1.5+0.9 i$ : (a) space-time plot of $|A|$ propagating in one dimension and $(b)$ phase portrait of wave patterns. 
Nonlinear Dynamical Regimes and Control of Turbulence through the Complex...

DOI: http://dx.doi.org/10.5772/intechopen.88053

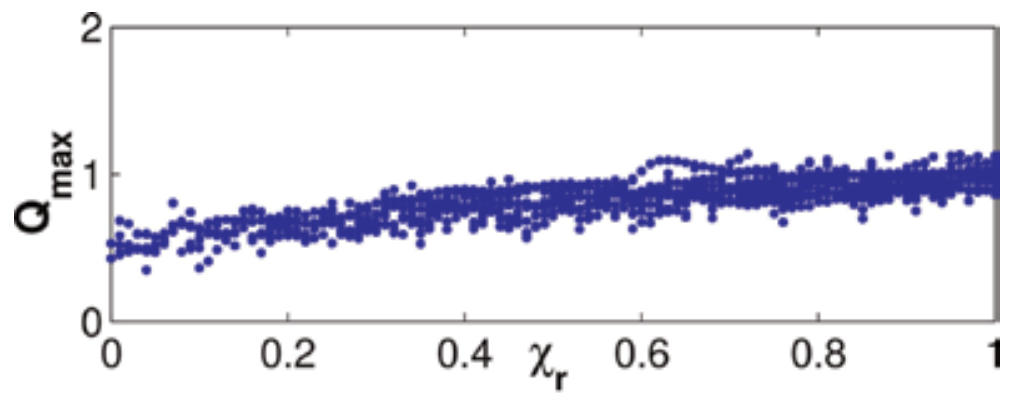

a)

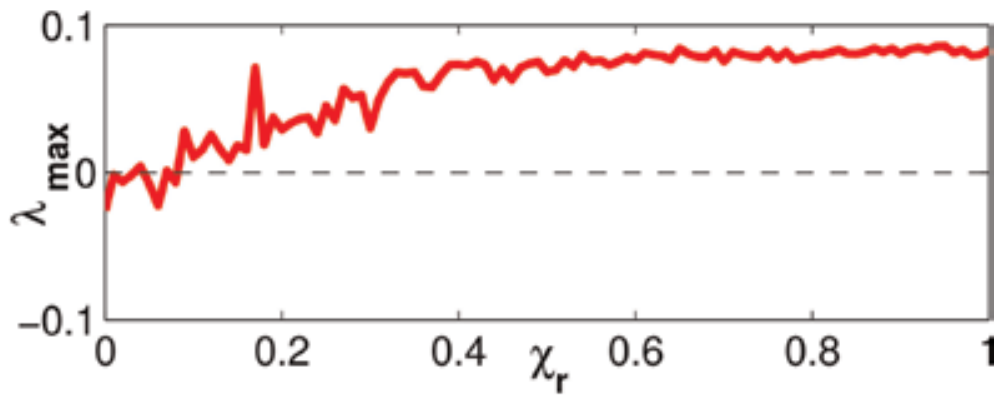

b)

Figure 26.

(a) Bifurcation diagram of $Q_{\max }$ and (b) largest Lyapunov exponent $\lambda_{\max }$ as function of $\chi_{r}$, without nonlinear gradient terms.

system changes its dynamical behavior with the presence of the nonlinear gradient terms. Figure 26a, which express the case without the nonlinear gradient terms, is obtained by taking repeatedly the maximum value of the energy function $Q_{\max }$ in a

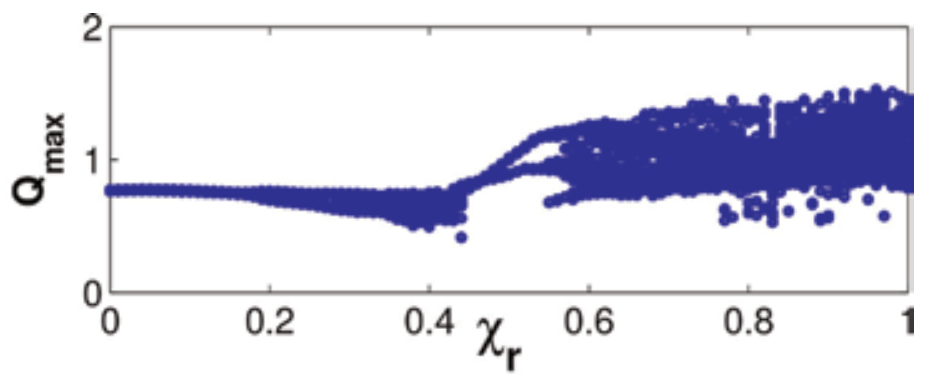

a)

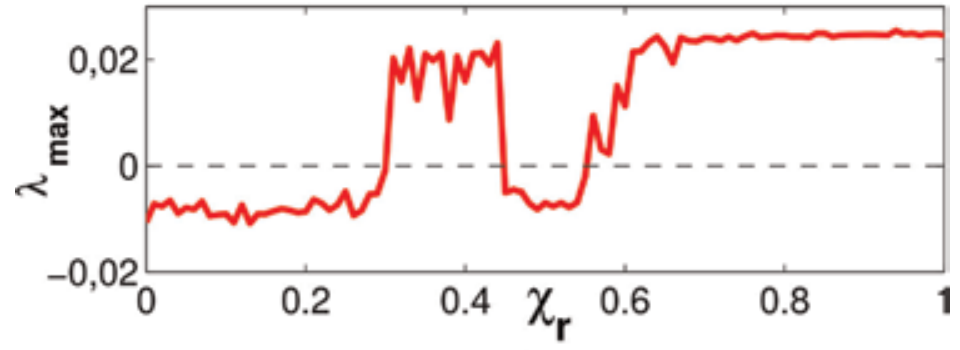

b)

Figure 27.

(a) Bifurcation diagram of $Q_{\max }$ and (b) largest Lyapunov exponent $\lambda_{\max }$ as function of $\chi_{r}$, with $q_{1}=0.1+0.1 i, q_{2}=0.2+0.2 i$, and $q_{3}=0.3+0.3 i$. 


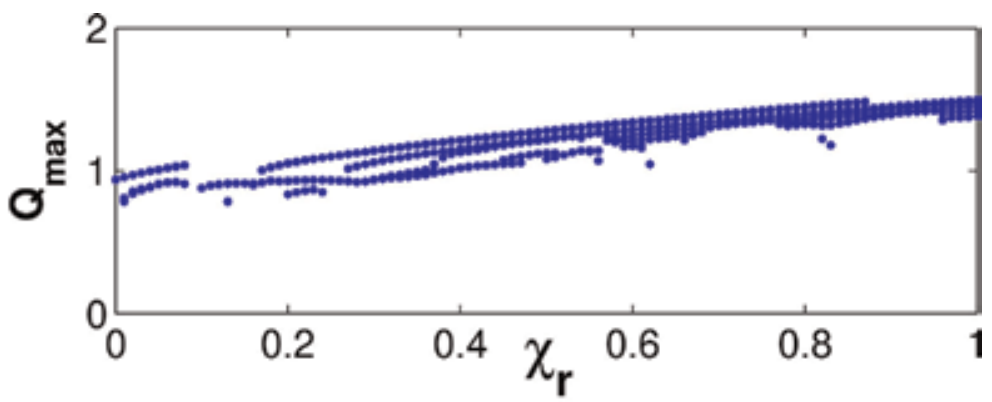

a)

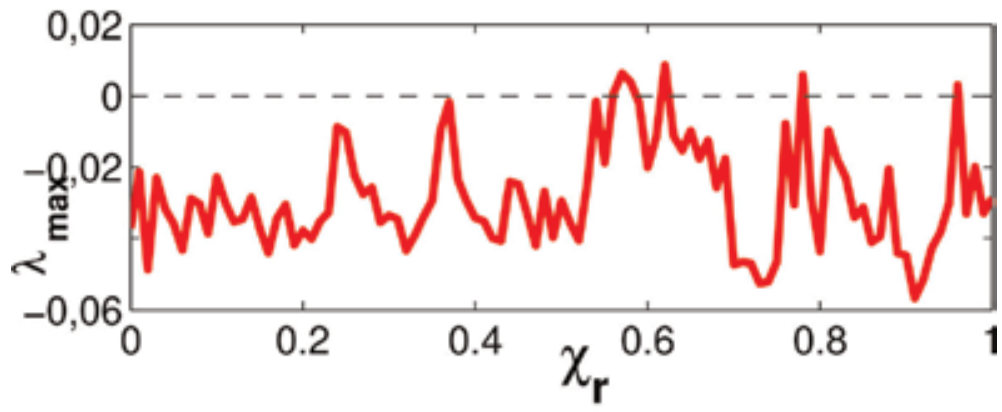

b)

Figure 28.

(a) Bifurcation diagram of $Q_{\max }$ and (b) largest Lyapunov exponent $\lambda_{\max }$ as function of $\chi_{r}$, with $q_{1}=0.7+0.7 i$.

given time interval at different times (well after the transient is dead); this is done for very many different values of the control parameter $\chi_{r}$. As can be seen, the system is briefly stable, and then, the instability is present in the whole system. In fact, if there is a unique $Q_{\max }$, then the system is stationary or periodic, while for finite continuous distribution of $Q_{\max }$ values, the behavior is either quasi-periodic or chaotic.

The Lyapunov exponent shown in Figure 26b indicates the dynamical behavior of the system and confirms the results. Figure 27 is obtained for increasing values of the nonlinear gradient terms. We observe several transitions between regular and chaotic states. In particular, there is a small stability part of the system in the range $\chi_{r} \in(0.0,0.3)$. Beyond the point $\chi_{r}=0.44$, the system becomes stable again until the value $\chi_{r}=0.55$, with another bifurcation point at $\chi_{r}=0.6$. On the other hand, the transition from regular to chaotic wave patterns is flat. In the plot of the Lyapunov exponent (see Figure 27b), the chaotic motions identified are validated by the positive values of $\lambda_{\max }$, while the stable region corresponds to the negative values of $\lambda_{\max }$. For the large value of the nonlinear gradient terms, the system becomes more and more stable as is seen in Figure 28.

\section{Conclusion}

The numerical investigation of the 1D quintic CGL equation in such systems represents a big topic in the understanding of many physical systems with pattern formation. Concerning the extended system, we have summarized in a phase diagram all the regimes that have been observed. On the phase diagram, we have the BFN line that divides the regions in two regions: the stable zone which contains laminar state and spatiotemporal intermittency regime and the unstable zone with 
chaotic regimes as phase turbulence, weak turbulence, and defect turbulence [49]. For the case of finite system, we have described from the 1D cubic-quintic CGL equation the effects of the boundaries on the waves traveling in a preferred direction. We have used the homogeneous boundary conditions, and the waves were nonlinear dissipative waves. We have studied the nature of convective or absolute instabilities of wave patterns. In our simulations, we have found new states that were similar from those obtained in the cubic CGL equation with homogeneous boundary conditions or with the Neumann boundary conditions. The presence of the quintic term has a large influence in the wave pattern. All the dynamic regimes observed have been summarized in a state diagram. The regimes as the global mode regime, turbulence regime observed in the secondary structures, and spatiotemporal intermittency regimes have been detected, but their shape and behavior are different depending on the sign of $c_{5}$. Such defects, holes, and spatiotemporal intermittency regime have been observed in the spiral wave pattern in the counterrotating Couette-Taylor system, in the Taylor-Dean system, and in Rayleigh-Bénard convection. These results were different from those that used periodic boundary conditions. The numerical investigation of the $1 \mathrm{D}$ cubic-quintic CGL equation with homogeneous boundary conditions for different values of the control parameters represents a big topic in the understanding of many physical systems with pattern formation. One of the great challenges is to control these instabilities. We have used three types of control. Many significant technological applications, such as mixing, optical fiber manufacture, and chemical reaction could crucially benefit from the control of instabilities leading to complex spatiotemporal dynamics. Firstly, we have proposed a method based on a nonlinear diffusion parameter control which lies to the modified cubic-quintic CGL equation in the extended system. In the first time, we have briefly presented the model equation of cubic-quintic CGL equation and summarized the dynamic regimes observed in the phase diagram of BFN. This method is based on the modification of the nonlinear coefficient term with the preservation of the intrinsic phase invariance of the original equation. By using hole solutions as initial conditions, we have simulated the modified cubic-quintic CGL equation. Then, spatiotemporal chaos regimes obtained with the original cubic-quintic CGL equation have been stabilized by using a nonlinear diffusion term. The results show that, the defect turbulence regime which is initially observed, become progressively stable by modifying the values of the nonlinear diffusion term, i.e., at the end, plane wave regime is observed. Then, by using another type of control called time-delay auto-synchronization, which consists in adding the feedback term to the CGL equation, the results were excellent. We have noticed by this method that one can control or avoid the spatiotemporal chaos observed into the system. The last method control used is the adding of the nonlinear gradient terms to the CGL equation called the lowest-order CGL equation. By considering also the wave patterns in the chaotic regions in particular in a defect turbulence regime, it was shown that the presence of the nonlinear gradient terms changes the dynamical behavior of the system; the chaos can disappear progressively in the domain. The fact that the nonlinear gradient terms can stabilize the system leads us to conclude that they can be considered as the stabilizing terms.

\section{Acknowledgements}

The work by CBT is supported by the Botswana International University of Science and Technology under the grant DVC/RDI/2/1/16I (25). CBT thanks the Kavli Institute for Theoretical Physics (KITP), University of California Santa Barbara (USA), for invitation. 


\section{Author details}

Joël Bruno Gonpe Tafo ${ }^{1}$, Laurent Nana ${ }^{1}$, Conrad Bertrand Tabi ${ }^{2 *}$ and Timoléon Crépin Kofané ${ }^{3}$

1 Laboratoire de Physique Fondamentale, Groupe Phénomènes Non Linéaires et Systèmes Complexes, UFD de Physique Fondamentale et Sciences de l'Ingénieur, Université de Douala, Douala, Cameroun

2 Botswana International University of Science and Technology, Palapye, Botswana

3 Laboratoire de Mécanique, Département de Physique, Faculté des Sciences, Université de Yaoundé I, Yaoundé, Cameroun

*Address all correspondence to: tabic@biust.ac.bw

\section{IntechOpen}

(C) 2020 The Author(s). Licensee IntechOpen. This chapter is distributed under the terms of the Creative Commons Attribution License (http://creativecommons.org/licenses/ by/3.0), which permits unrestricted use, distribution, and reproduction in any medium, provided the original work is properly cited. (c) BY 
Nonlinear Dynamical Regimes and Control of Turbulence through the Complex...

DOI: http://dx.doi.org/10.5772/intechopen.88053

\section{References}

[1] Cross M, Hohenberg P. Pattern formation outside of equilibrium. Reviews of Modern Physics. 1993;65:851

[2] Lega J. Traveling hole solutions of the complex Ginzburg-Landau equation. Physica D: Nonlinear Phenomena. 2001; 152:269

[3] Coullet P, Gil L, Lega J. Defectmediated turbulence. Physical Review Letters. 1989;62:1619

[4] Nana L, Ezersky AB, Abcha N, Mutabazi I. Dynamics of spatiotemporal defects in the Couette-Taylor flow: Comparison of experimental and theoretical results. Journal of Physics: Conference Series. 2008;137:012006

[5] Kodama Y, Hasegawa A. Generation of asymptotically stable optical solitons and suppression of the Gordon-Haus effect. Optics Letters. 1992;17:31

[6] Lee KJ, McCormick WD, Ouyang Q, Swinney HL. Experimental observation of self-replicating spots in a reactiondiffusion system. Nature (London). 1994;369:215

[7] Bramwell ST, Holdsworth PCW, Pinton JF. Universality of rare fluctuations in turbulence and critical phenomena. Nature (London). 1998; 396:552

[8] Kuramoto Y. Chemical Oscillations, Waves, and Turbulence. Berlin: Springer; 1984

[9] Kim M, Bertram M, Pollmann M, von Oertzen A, Mikhailov AS, Rotermund $\mathrm{HH}$, et al. Controlling chemical turbulence by global delayed feedback: Pattern formation in catalytic co oxidation on pt(110). Science. 2001; 292:1357

[10] Sinha S, Pande A, Pandit R. Defibrillation via the elimination of spiral turbulence in a model for ventricular fibrillation. Physical Review Letters. 2001;86:3678

[11] Aranson IS, Kramer L. The world of the complex Ginzburg-Landau equation. Reviews of Modern Physics. 2002;74:99

[12] Lega J, Fauve S. Traveling hole solutions to the complex GinzburgLandau equation asperturbations of nonlinear Schrödinger dark solitons. Physica D: Nonlinear Phenomena. 1997; 102:234

[13] van Hecke M. Building blocks of spatiotemporal intermittency. Physical Review Letters. 1998;80:1896

[14] Descalzi O, Gutiérrez P, Tirapegui E. Localized structures in nonequilibrium systems. International Journal of Modern Physics C. 2005; 16:1909

[15] Gonpe Tafo JB, Nana L, et L, Kofane TC. Dynamics of a traveling hole in onedimensional systems near subcritical bifurcation. The European Physical Journal Plus. 2011;126:105

[16] Nana L, Ezersky AB, Mutabazi I. Secondary structures in a onedimensional complex Ginzburg-Landau equation with homogeneous boundary conditions. Proceedings of the Royal Society A. 2009;465:2251

[17] Descalzi O, Brand HR. Influence of Dirichlet boundary conditions on dissipative solitons in the cubic-quintic complex Ginzburg-Landau equation. Physical Review E. 2010;81:026210

[18] Gonpe Tafo JB, Nana L, Kofane TC. Nonlinear structures of traveling waves in the cubic-quintic complex GinzburgLandau equation on a finite domain. Physica Scripta. 2013;87:065001 
[19] Schuster H. Handbook of chaos control: Foundations and applications. 1st ed. Weinheim: Wiley-VCH; 1999

[20] Gonpe JB, Nana L, Kofane TC. Nonlinear diffusion control of defect turbulence in cubic-quintic complex Ginzburg-Landau equation. The European Physical Journal Plus. 2012; 127:75

[21] Roy R, Murphy T, Maier T, Gills Z, Hunt E. Dynamical control of a chaotic laser: Experimental stabilization of a globally coupled system. Physical Review Letters. 1992;68:1259

[22] Hunt E. Stabilizing high-period orbits in a chaotic system: The diode resonator. Physical Review Letters. 1991;67:1953

[23] Ditto W, Rauseo S, Spano M. Experimental control of chaos. Physical Review Letters. 1990;65:3211

[24] Ott E, Grebogi C, Yorke JA. Controlling chaos. Physical Review Letters. 1990;64:1196

[25] Pyragas K. Continuous control of chaos by self-controlling feedback. Physics Letters A. 1992;170:421

[26] Pyragas K, Tamaševičius A. Experimental control of chaos by delayed self-controlling feedback. Physics Letters A. 1993;180:99

[27] Gonpe Tafo JB, Nana L, Kofane TC. Time-delay autosynchronization control of defect turbulence in the cubic-quintic complex Ginzburg-Landau equation. Physical Review E. 2013;88:032911

[28] Deissler RJ, Brand HR. The effect of nonlinear gradient terms on breathing localized solutions in the complex Ginzburg-Landau equation. Physical Review Letters A. 1998;81:3856

[29] Yomba E, Kofane TC. Solutions of the lowest order complex Ginzburg-Landau equation. Journal of the Physical Society of Japan. 2000;69:1027

[30] Gonpe Tafo JB, Nana L, Kofane TC. Effects of nonlinear gradient terms on the defect turbulence regime in weakly dissipative systems. Physical Review E. 2017;96:022205

[31] Bottin S, Lega J. Pulses of tunable size near a subcritical bifurcation. European Physical Journal B. 1998;5:299

[32] Thual O, Fauve S. Localized structures generated by subcritical instabilities. Journal of Physiology, Paris. 1988;49:1829

[33] Chaté H. Spatiotemporal intermittency regimes of the onedimensional complex Ginzburg-Landau equation. Nonlinearity. 1994;7:185

[34] Newell AC, Nazarenko S, Biven L. Wave turbulence and intermittency. Physica D: Nonlinear Phenomena. 2001; 152:550

[35] Lvov YV, Tabak EG. Hamiltonian formalism and the Garrett-Munk spectrum of internal waves in the ocean. Physical Review Letters. 2001;87:168501

[36] Coullet P, Elphick C, Repaux D. Nature of spatial chaos. Physical Review Letters. 1987;58:431

[37] Tobias SM, Knobloch E. Breakup of spiral waves into chemical turbulence. Physical Review Letters. 1998;80:4811

[38] Aranson IS, Aranson L, Kramer L, Weber A. Stability limits of spirals and traveling waves in nonequilibrium media. Physical Review A. 1992;46: R2992

[39] Voss HU, Kolodner P, Abel M, Kurths J. Amplitude equations from spatiotemporal binary-fluid convection data. Physical Review Letters. 1999;83: 3422 
[40] Bot P, Mutabazi I. Dynamics of spatio-temporal defects in the TaylorDean system. European Physical Journal B. $2000 ; 13: 141$

[41] Beta C, Bertram M, Mikhailov AS, Rotermund HH, Ertl G. Controlling turbulence in a surface chemical reaction by time-delay autosynchronization. Physical Review E. 2003;67:046224

[42] Beta C, Mikhailov AS. Controlling spatiotemporal chaos in oscillatory reaction-diffusion systems by time delay auto synchronization. Physica D: Nonlinear Phenomena. 2004;199:173

[43] Falcke M, Engel H, Neufeld M. Cluster formation, standing waves, and stripe patterns in oscillatory active media with local and global coupling. Physical Review E. 1995;52:763

[44] Falcke M, Engel H. Pattern formation during the $\mathrm{CO}$ oxidation on $\mathrm{Pt}(110)$ surfaces under global coupling. The Journal of Chemical Physics. 1994; 101:6255

[45] Duan J, Holmes P. On the Cauchy problem of a generalized GinzburgLandau equation. Nonlinear Analysis. 1994;22:1033

[46] Barten W, Lücke M, Kamps M, Schmitz R. Convection in binary fluid mixtures. II. Localized traveling waves. Physical Review E. 1995;51:5662

[47] Lücke M, Barten W, Kamps M. Convection in binary mixtures: The role of the concentration field. Physica D: Nonlinear Phenomena (Amsterdam, Netherlands). 1992;61:183

[48] Akhmediev N, Soto-Crespo JM. Strongly asymmetric soliton explosions. Physical Review E. 2004;70:036613

[49] Burke J, Knobloch E. Homoclinic snaking: Structure and stability. Chaos. 2007;17:037102
[50] Sherratt JA, Smith MJ. Transition to spatiotemporal chaos via stationary branching shocks and holes. Physica D: Nonlinear Phenomena (Amsterdam, Netherlands). 2012;241:1671

[51] Urzagasti D, Laroze D, Pleiner H. Localized chaotic patterns in weakly dissipative systems. The European Physical Journal Special Topics. 2014; 223:141 



\title{
Ubiquitous Filtering for Nonlinear Problems
}

\author{
Valeri Kontorovich and Fernando Ramos-Alarcon
}

\begin{abstract}
This chapter develops and extends the general theoretical results, previously published in the chapter "Nonlinear filtering of weak chaotic signals", and presents detailed implementations of a computationally simple, robust (filtering fidelity almost insensitive to changes of the desired input signal properties) and rather precise approach for the filtering of weak signals of different physical nature (biological, seismic, voice, etc.) in presence of white Gaussian noise. The implementations rely on non-linear filtering techniques that in general can be considered as either one-moment or multi-moment, in the sense that they operate with a single sample (instantaneous fashion) or with various adjacent samples (non-instantaneous fashion). Chaotic modeling of the real input signals allows achieving an almost ubiquitous filtering approach with a computationally simple implementation. Application of the linearization strategies (for both one and twomoment filtering) provide, additionally, "invariance" of the processing algorithms to variations on the nature and statistics of the input signals.
\end{abstract}

Keywords: system identification toolbox, linearized models, weak chaos, quasi-linear filtering algorithms

\section{Introduction}

The signal filtering plays a fundamental role in the design of signal processing algorithms for many problems, that is, the first step is to remove (to filter) the background noise from the input (incoming) signal, and the second step is to perform the corresponding signal processing $[1,2]$. In this sense, the filtering approach based on the theory of dynamic systems [3-5] pops up immediately as one of the possible ways to address this issue. The dynamic filtering approach, such as classic linear Kalman filtering, has been applied for many problems long ago [6] and recently as well [7]. However, in the following the dynamic filtering is proposed adopting a different (nonlinear) angle $[8,9]$, namely, using signals from nonlinear chaotic attractors as a model for the desired signals arriving at the filtering structure. The modeling of real phenomena using chaos has been used for more than 50 years, and there is a wide range of scientific and practical applications, such as seismology [10-12], statistical theory of communication $[13,14]$, control, geophysics, biomedical telemetry $[15,16]$ under water signal processing [17], and many other areas related to applied physics as well [18].

When the signals of interest are significantly weak (smaller than the background additive white Gaussian noise, AWGN), the problem is far from trivial. The 
following material will show the effectiveness of using a dynamic nonlinear strategy (introduced in $[4,5]$ ) for filtering signals, belonging to different types of real phenomena, which are modeled through components of chaotic attractors, all this in presence of strong AWGN, which concretely means a signal to noise ratio, SNR, $<1(<0 \mathrm{~dB})$. Note that even though weak signals are treated in the literature, $[10,12]$, their processing is not addressed from the dynamic filtering point of view, and therefore (in our opinion) optimum fidelity solutions are still required.

In the following the term, effective filtering is used to indicate high precision which is evaluated considering values of the normalized mean square error, NMSE, $<1 \%$ (in the following the normalization of the MSE will be considered in relation to the variance of the desired input signal). In the regular practice, there might be several precision measures corresponding to each specific filtering scenario. The use of the NMSE, or RMSE (root mean square error for nonstationary scenarios), as a measure of precision (fidelity) for filtering is well established from the statistical theory [19], and so, it can be considered as "universal" because its formal definition is the same irrespectively to specific filtering scenarios $[1,2]$. Also note that the case of small values of the NMSE might adequately correspond to concrete practical criteria of fidelity $[3,19]$.

The proposed strategy is robust but not in the sense used in control theory, where the term "robust" means that the filter's structure is invariant to a priori unknown features of the input signals. The proposed chaotic filtering is considered as robust in the sense that its fixed structure and fidelity are almost invariant to signals from rather different filtering scenarios, which in the following correspond to seismic signals, heart beat signals, voice-like signals, and radio frequency interference (RFI) signals. Actually one can see that such invariance makes the filtering "ubiquitous."

For conditions when the SNR $\leq 0 \mathrm{~dB}$, the term "weak chaotic signals" will be used, keeping in mind that chaos modeling is applied for the abovementioned filtering scenarios. Chaos modeling might be immensely useful because almost all quasi-optimum filtering algorithms (which formally are nonlinear but are essentially quasi-linear) show rather high precision in the sense of low NMSE $(\leq 1 \%)$ and exhibit low computational complexity among other benefits. These properties were broadly discussed in $[8,9]$, where theoretical proofs can be found. The material presented here contains some experimental applications, for rather different scenarios that apply and extend the ideas presented in [9], and so, both have to be considered together. In [9] it was impossible for the authors to include the present material, not even partially, for the lack of space.

\section{Extraction of some theoretical principles}

\subsection{Chaotic modeling and filtering}

Let us assume that a chaos vector process $x(t)$ can be generated from the following ordinary differential equation (ODE) of certain attractor [18]:

$$
\dot{x}=F(x, t)
$$

where the initial condition is $\boldsymbol{x}\left(t_{0}\right)=\boldsymbol{x}_{0}$ and $\boldsymbol{F}(\bullet)$ is a vector function, which (for any real application) is a priori unknown (together with the initial conditions) and needs to be somehow identified beforehand and moreover is usually time varying. It is worth mentioning that the identification problem for $\boldsymbol{F}(\bullet)$ has attracted a lot of interest in the last decades, but being rather complex, it has not 
been accomplished so far, at least to the author's knowledge. The reason behind this is the identification of Eq. (1) is an identification of the "inertial vector nonlinear system" which does not have an unique solution and can be formulated only for a previously defined class of nonlinear systems; the complexity of this task has been addressed elsewhere $[8,9,18,20]$ and will not be considered in the following. As examples for $\boldsymbol{F}(\bullet)$, which will be used in the following, there are the equations for the chaotic attractors corresponding to Rossler, Lorenz, and Chua types $[8,9]$ :

Continuous time:

Discrete time:

$$
\begin{array}{rlrl}
\dot{x}(t) & =-y(t)-z(t) & x_{k+1} & =x_{k}+T_{s}\left(-y_{k}-z_{k}\right) \\
\text { Rossler } \dot{y}(t) & =x(t)+0.2 y(t) & y_{k+1} & =y_{k}+T_{s}\left(x_{k}+0.2 y_{k}\right) \\
\dot{z}(t) & =0.2-z(t)(5.7-x(t)) & z_{k+1} & =z_{k}+T_{s}\left(0.2-z_{k}\left(5.7-x_{k}\right)\right) \\
& & & x_{k+1}=x_{k}+T_{s}\left(10\left(x_{k}-y_{k}\right)\right) \\
\dot{x}(t) & =10(x(t)-y(t)) & & \\
\text { Lorenz } \dot{y}(t) & =28 x(t)-y(t)+x(t) \cdot z(t) & y_{k+1}=y_{k}+T_{s}\left(28 x_{k}-y_{k}+x_{k} \cdot z_{k}\right) \\
\dot{z}(t) & =-\frac{8}{3} z(t)+x(t) \cdot y(t) & z_{k+1}=z_{k}+T_{s}\left(-\frac{8}{3} z_{k}+x_{k} \cdot y_{k}\right) \\
\text { Chua } \dot{y}(t) & =x(t)-y(t)+z(t), & y_{k+1} & =y_{k+1}+T_{s}\left[x_{k}-y_{k}+z_{3}\right], \\
\dot{z}(t) & =-14.3 y(t) . & z_{k+1} & =z_{k+1}+T_{s}\left[-14.3 y_{k}\right],
\end{array}
$$

where $U\left(x_{k}\right)=m_{1} x_{k}+\frac{1}{2}\left(m_{0}-m_{1}\right)\left[\left|x_{k}+1\right|-\left|x_{k}-1\right|\right], m_{0}=-\frac{1}{7}$, and $m_{1}=\frac{2}{7}$ and $T_{S}$ is the sampling time.

In order to neglect the uncertainty effects of the initial conditions, at least for real data filtering, the approach used in [21], based on the fundamental statement of statistical dynamics for deterministic systems related to Kolmogorov and Max Born [18], together with the introduction of the so-called additive "process noise" in Eq. (1), can be applied. The latter transforms the ODE Eq. (1) into a stochastic differential equation (SDE) [20]. The transformation of Eq. (1) into SDE is relevant for the following material.

The equation for strange attractor Eq. (1) can be transformed into the equivalent stochastic form as a stochastic differential equation (SDE), which "generates" the n-dimensional Markov stochastic process [18, 20]:

$$
\dot{\boldsymbol{x}}=\boldsymbol{f}(\boldsymbol{x}(t))+\boldsymbol{\varepsilon} \xi(t)
$$

where $\boldsymbol{f}(\boldsymbol{x}(t))$ is identical to $\boldsymbol{F}(\boldsymbol{x}(t))$ from Eq. (1). The influence of a weak external source of white noise is denoted by $\xi(t)$; the noise intensities are given in a matrix form $\varepsilon=\left[\varepsilon_{i j}\right]^{n \times n}$.

The solutions proposed hereafter might be encountered from the structural analysis of the quasi-optimum filtering algorithms for weak chaos in presence of AWGN $[8,9]$ and are synthesized in the following for convenience.

When one uses the SDE Eq. (5) as a model for chaos, the first strategy that comes up immediately to mind is the nonlinear filtering of chaotic signals which was rigorously developed in $[8,9]$. The kernel presented in [8] is the StratonovichKushner equation (SKE) $[4,5]$, which allows to describe the dynamic equation for the a posteriori probability density function for the chaos $x(t)$. For the filtering with this generalized approach, some additional information from the received aggregate signal has to be incorporated on several sequential time instants, i.e., the 
information has to be considered in the block manner by aggregating data, in our case, from several time instants (multi-moment processing). Multi-moment algorithms are carried out through the generalization of the Stratonovich-Kushner Equations for the corresponding multi-moment data. In this way the resulting heuristics are not arbitrary; they are actually generalized heuristics from the standard one-moment SKE. All this gives hope that one can achieve rather good MSE for successively lower thresholds of the SNR using an algorithm with rather low complexity.

Note that the time evolution for the a posteriori PDF for $x(t)$ is completely described by the SKE, but, unfortunately, it does not provide exact analytical solutions. There are very few exceptions: linear SDE Eq. (4) which yields the wellknown Kalman filtering algorithm [4, 22] and some others [4, 5]. Due to this the nonlinear filtering algorithms are practically always simplified, as quasi-optimum or even quasi-linear $[4,5]$. In practical applications quasi-linear filters are broadly applied [4, 5].

One might wonder, what is the reason behind the application of chaotic modeling for weak signal filtering? The kernel for this lies in the "singular" properties of the solution of the SKE (see Eqs. (9) and (10) in reference [9]) for the dynamic ODE for chaos Eq. (1), when the solution of the SKE is almost "tuned" to the deterministic chaos from Eq. (1) without any dependence to the SNR [8]. Sure, this statement has to be interpreted as a qualitative explanation for the solution properties of the SKE, and it is almost true for the behavior of the quasi-linear algorithms as well $[8,9]$.

The following is a list of several quasi-linear filtering algorithms for chaotic signals, based on so-called "Local Gaussian Approximation Approach for the a posteriori PDF" $[4,9]$, which was found as rather opportunistic for real-time implementations:

\section{Extended Kalman filter (EKF)}

2. Unscented Kalman filter (UKF)

3. Kalman quadrature filter (KQF)

4. Gauss-Hermite quadrature filter (GQF)

\section{Conditionally optimum filter}

All these algorithms certainly show different filtering precision for a fixed SNR and completely different computational complexity for a fixed filtering fidelity. So, in the selection of a concrete filtering algorithm for a concrete scenario, one has to consider as possible selecting criteria the MSE (NMSE and RMSE) together with the computational complexity.

Theoretically, the simplest way to get a comparative analysis of the abovementioned algorithms for the case of weak chaos filtering is in the framework of the so-called stochastic equivalent approximation of the observable component of the chaotic attractor, considered as an adequate model of the real process for filtering.

\subsection{Stochastic differential equation of the first order (SDE-1)}

The idea of the stochastically equivalent dynamic system (or SDE) was presented for the first time by Stratonovich and Kushner in [5] and extensively 
developed for many real scenarios [20]. Let a chaotic attractor with certain observable component in Eq. (1) together with its stochastic characterization be a model of the input data. One might consider a random process, generated by a stochastic differential equation of the first order (SDE-1), and name it as a stochastic equivalent as long as it has the same probability density function (PDF) and the same covariance function as the observable component. So, if one assumes that the stochastic equivalent (through the solution of the scalar SDE-1) is an adequate approach to substitute the model of the real phenomena (in the form of an observable component of the multidimensional chaotic attractor), then the actual model is $[5,20]$ :

$$
\dot{x}=f(x)+\sqrt{K} \xi(t)
$$

where the local characteristics, here denoted as $K_{2}(x)$ and $K_{1}(x)$ for Eq. (6), are $K_{1}(x)=f(x), K_{2}(x)=K$, and $\xi(t)$ is AWGN with unitary intensity $[5,20,23] .{ }^{1}$ If the input signal for filtering is:

$$
y(t)=S(x, t)+n_{0}(t)
$$

where $n_{0}(t)$ is AWGN with intensity $N_{0}$, then applying the standard procedure of local Gaussian approximation approach for the a posteriori PDF (which for this particular case includes Taylor series representation for all nonlinearities and also includes the PDF exponent and is limited to only quadratic terms at the SKE $[4,5]$ ), one can get the following quasi-optimum filtering algorithms:

$\dot{\hat{x}}=f(\hat{x})+\frac{2 P_{11}(t)}{N_{0}}[y(t)-S(\hat{x}, t)] \frac{\partial S(\hat{x}, t)}{\partial x} \dot{P}_{11}(t)=\frac{K}{2}+\frac{2 \partial f(\hat{x})}{\partial x} P_{11}-\frac{2}{N_{0}}\left[\frac{\partial S(\hat{x})}{\partial x}\right]^{2} P_{11}^{2}$

where $\hat{x}(t)$ and $P_{11}(t)$ are a posteriori mean (estimated value) and variance (error) of filtering, respectively. Applying then the well-known standard EKF synthesis procedure [4] for Eqs. (6) and (7), one can also easily obtain the algorithm Eq. (8). It is worth mentioning that the difference between the above-listed algorithms for the local Gaussian approximation depends only on the way the localization of the instantaneous estimation of $x(t)$ is chosen (as it will be commented in the following).

For the case of high filtering accuracy, all other algorithms that apply local Gaussian approximation [8] can be successfully approximated by the EKF, because the true value of the filtered process and the reference point for application of the Gaussian approximation are obviously very "close."

The algorithm Eq. (8) is related to the so-called one-moment (1MM) regime which is classical for the EKF. In the $1 \mathrm{MM}$ regime during each processing cycle, one sample from one instant of time is processed (instantaneous processing). The $2 \mathrm{MM}$ regime was exhaustively presented at $[8,9]$ as a special case of multi-moment filtering and could be easily reviewed by the interested reader. In the $2 \mathrm{MM}$ regime during each cycle, two samples from two instants of time are processed (non-instantaneous processing). The main parameter for $2 \mathrm{MM}$ algorithm is " $\rho$ ", which is the correlation coefficient between two adjacent samples of the processing algorithm.

\footnotetext{
${ }^{1}$ One can see that for the SDE Eq. (5) the Stratonovich and Ito forms for $K_{1}(x)$ and $K_{2}(x)$ are identical.
} 
Let us stress here that the concept of stochastic equivalence of the observable component together with the SDE-1 was used only to make our statements in a simple and "friendly" way and to provide computationally simple algorithms. For the general case of the vector SDE (vector ODE) Eq. (1), when the stochastic equivalence in the above presented form cannot be applied, because the high-order statistics (HOS) play a significant role [4], all of the above qualitative comments are true as well; the term $\frac{\partial S(\hat{x}, t)}{\partial x}$ at Eq. (8) has to be substituted by the Jacobian matrix, which is usually considered as a "linearization coefficient" at the point $x=\hat{x}$ [4]. It follows that the synthesis approach for the filtering algorithms (in the framework of the local Gaussian approximations for the a posteriori PDF) can be considered as an instantaneous (miscellaneous) linearization approach.

\subsection{Computational complexity}

The next issue, which has to be analyzed here, is the computational complexity of the quasi-linear algorithms. This subject is crucial for the applications addressed in the next section.

For the general case, when EKF, UKF, GHF, and QKF algorithms are applied considering Chua, Lorenz, and Rössler attractor signals as desired input signals, the computational complexity for the processing is presented in the following table, where all operations, additions (subtractions), multiplications (divisions), Cholesky decompositions, Jacobian calculation (linearization), and nonlinear propagation are included.

From Table 1, it can be easily seen that UKF involves the bigger complexity, while EKF seems to be the simplest algorithm. However, the linearization process performed by the Jacobian calculation involves partial derivatives. For that reason, and depending on the mathematical model of the attractor, the EKF may not always be the fastest algorithm. It follows from Table 1 that the EKF algorithm provides the simplest implementation. Moreover, as it will be shown in the following section, the EKF fidelity for weak signal detection is acceptable in all practical cases.

Together with the simple theoretical analysis, the EKF can be considered as an opportunistic approach for applications (see the next section as well).

But one has to notice that for the robust (ubiquitous) solution and applications (see above) the EKF has to be additionally modified by the following heuristics. One can assume, as an alternative to the quasi-linear EKF algorithms, where the linearization is instantaneously updated, that the robust solution for the EKF applications might be found if a "fixed linearization" (with predefined linearization matrix) is used instead of an "instantaneous" one. It actually means that instead of the EKF, the standard Kalman filtering (SKF) approach is applied [3-7], and obviously one has to admit some "losses" in the filtering accuracy for this case. At the same time, it

\begin{tabular}{lcccc}
\hline & EKF & UKF & GHF & QKF \\
\hline Additions & 8 & 50 & 25 & 25 \\
\hline Multiplications & 15 & 77 & 33 & 40 \\
\hline Cholesky decomposition & 1 & 2 & 2 & 2 \\
\hline Nonlinear propagation & 0 & 15 & 21 & 6 \\
\hline Jacobian calculation & 1 & 0 & 0 & 0 \\
\hline
\end{tabular}

Table 1.

Computational complexity. 
has to be taken into account that the local Gaussian approximation of the a posteriori PDF assumes that actually all the model components are almost linear and therefore the accuracy losses might be rather moderate.

These filtering assumptions seem to be valid for several practical problems such as interference mitigation, seismology, biomedical telemetry, etc. For weak chaotic signals, in this condition it is possible to consider the EKF with "linear" Jacobian matrix or even SKF instead of the EKF, which additionally simplifies the problem. To obtain the linearization procedure, i.e., operate with a linear matrix $A(t)$ at Eq. (1), that comes from the linear approximation of the attractor's model for chaos, one can use the broadly applied "system identification toolbox" (SIT), [24, 25], which actually provides a solution for $A(t)$ with the spectral properties of the real data. It is worth mentioning that the way how the SIT identifies the linear matrix $A(t)$ follows from four "canonical representations" for the linear systems stated at [3].

Once more, it is only an approximation of an instantaneous linearization procedure required by the quasi-optimum filtering using local Gaussian approximation, but it gives a hope that for a high filtering precision NMSE of about $1 \%$ or less (see comments above) the filtering precision losses (by use of the mentioned identification approach) might be moderate and rather acceptable for practice (see also results of the experimental setup). As a final comment, let us note that the "linearization ideology," as an approach, is rather common (see the references already cited above) for quasi-optimum filtering algorithms with varying input data.

\section{Results and discusion}

The aim of this section is twofold and it will be considered separately. On one side the aim is to show that the stochastic equivalent approach (SDE-1) is efficient and has good accuracy for filtering purposes, taking the sufficiently nonlinear Chua attractor Eq. (4) as the most attractive example. On the other side, the aim is to illustrate the efficiency of the proposed methodology when it is applied to several real-world signals, of absolutely different physical nature, namely, seismic signals, electrocardiogram (ECG) signals, voice-like signals, and RFI signals. These experimental settings have been associated to nonlinear chaotic signals [10-12, 15, 26, 27], and very often, the scenario of such kind of signals includes a strong AWGN background, and so the desired signals are rather weak.

An experimental real-time test bed was developed, containing block generators for the AWGN, the EKF estimation (with their SDE-1 equivalents), the SKF estimation (with the linearization matrix coefficients evaluated from the SIT block), and the real input signals. The chaos EKF segment is a discrete implementation of the EKF which internally contains the discrete version of the equations for the strange attractors of Rossler, Lorenz, and Chua. It also performs a linearization by calculating the Jacobian in each processing cycle. For each signal setting, one of the attractor components ( $\mathrm{x}, \mathrm{y}$ or $\mathrm{z}$ ) has to be adapted as a possible signal model.

For this purpose, first, the sampling time of the chaotic discrete equations is varied so as to achieve a "match" between the temporal variations of the selected attractor component and the desired signal (make the time scales as close as possible). Second, the desired signal is normalized in relation to the mean and variance of the attractor component. The material of $[8,9]$ shows that the $x$-component of the three strange attractors might be suitable for modeling the signals from the experimental settings.

The SKF segment is a discrete implementation of the standard Kalman filter which in this case is tridimensional in order to make a fair comparison with the tridimensional EKF. In this segment the linearization matrix is obtained from the 


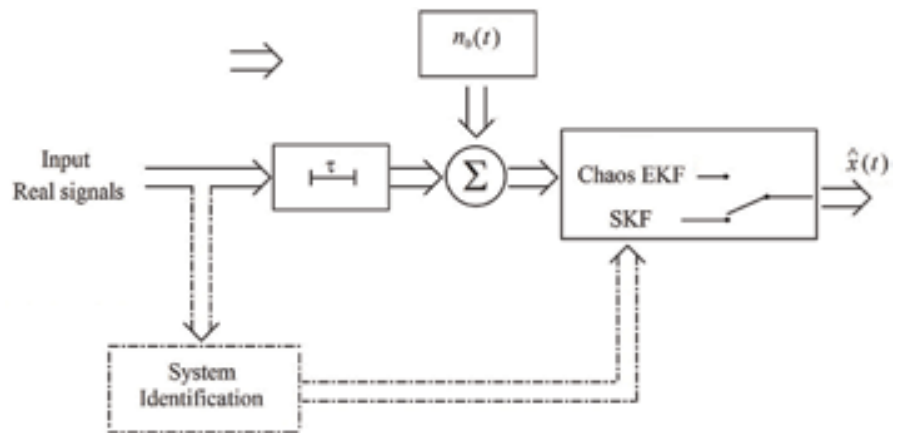

Figure 1.

Block diagram of the experimental test bed.

experimental signal (seismic, EGG, voice-like) using MATLAB's SIT. The matrix evaluation is made offline, calling the MATLAB's command "ident." Once the signal is loaded in the workspace, the identification is made selecting the option "state space models" [3,22] for the tridimensional case. The program offers three estimation options, and at the end it yields the confidence percentage for the selected estimation option. It was found experimentally that the PEM option (prediction error method) gives the best confidence for the estimated matrix. Note that for a fixed scenario from the real life, the matrix should be evaluated for each incoming signal offline before the signal processing is done (to obtain information both a priori and (or) from experimental data) as it is illustrated in Figure $\mathbf{1}$ by introducing a "virtual" delay " $\rho$," which means the separation in time of the matrix identification and filtering procedure; as the signals are stationary, the identification made for a large vector signal will suffice for any short vector signal. This experimental strategy is shown at Figure 1.

In the following, the experimental results apply the $1 \mathrm{MM}$ and $2 \mathrm{MM}$ filtering strategies. The $2 \mathrm{MM}$ strategy requires for its processing the correlation between two samples which in our case was set to $\rho=0.85$. The 2MM shows a bit better NMSE values as it is intuitively expected. For the scenario of seismic signals, it was not possible to calculate the linearization matrix from the SIT, as the signals are not tractable (limited signal durations for the spectral analysis). For all filtering scenarios, a weak process noise value $(Q)$ has been introduced (EKF and SKF) in order to exclude the uncertainty of the initial conditions and is indicated in the corresponding tables.

\subsection{Experiment one}

This experiment shows the efficiency of the stochastic equivalent SDE-1 for filtering. For illustration purposes, the intended signal here is the $\mathrm{x}$-component of the chaotic attractor from Chua. It is worth noticing that upon taking $x(t)$ in Eq. (4) as the observable component, the correspondent PDF is bimodal due to the function $\mathrm{U}(\cdot)$ [20]. The statistically equivalent SDE-1 for the case of Chua's x-component can be obtained straightforward from Eq. (8) $[8,9]$ :

$$
\dot{\hat{x}}_{1}=-2 K \hat{x}_{1}\left(p_{1}+q_{1}\right)+\frac{2\left(y(t)-\hat{x}_{1}\right)}{N_{0}} \quad \dot{\hat{P}}_{11}=-\frac{K}{2}+\frac{\hat{P}_{11}^{2}}{N_{0}}+4 K\left(p_{1}+q_{1}\right) \hat{P}_{11}
$$

where $p_{1}=3.5$ and $q_{1}=1.5$. 
Figure 2 shows the result for the NMSE. The dotted line corresponds to the SDE-1 filtering according to Eq. (9) and the continuous line to the 1MM 3D EKF using Eq. (4) in (A3), and in both cases the input signal is the Chua's $x$-component. The reason for doing this is that when one filters the input signal (Chua's x-component) using the Chua's Eq. (4), it is almost the best one can do (quasi-optimum solution), and that is why it gives the most adequate benchmark. From Figure 2 it is possible to see that there are some very moderate losses due to the use of the SDE-1 methodology, as it is logically expected; however the NMSE for the SDE-1 does not differ too much from the 1MM 3D EKF, and so the SDE-1 approach offers almost the best accuracy.

The following examples are devoted to the filtering of real data, which obviously differ from the theoretical chaos. The NMSE will increase because there is a mismatch between the input signal and the "chaotic signal component" from the filtering algorithm. This "mismatch" as it was mentioned above can be "compensated" by introducing a process noise with intensity $\mathrm{Q}$, in the filtering structure (A3).

\subsection{Experiment two: fetal electrocardiogram signals (FECG)}

The experimental data were obtained from a database ATM at PhysioNet [28]. The signal for this experiment corresponds to a baby's heart in fetal stage at the 36th week of the pregnancy cycle. For an SNR $=-3 \mathrm{~dB}$, Figure 3 shows the original signal and the filtered signal using $1 \mathrm{MM}$ EKF with Rossler $\mathrm{x}$-component as a model. Full results for the NMSE are shown in Table 2.

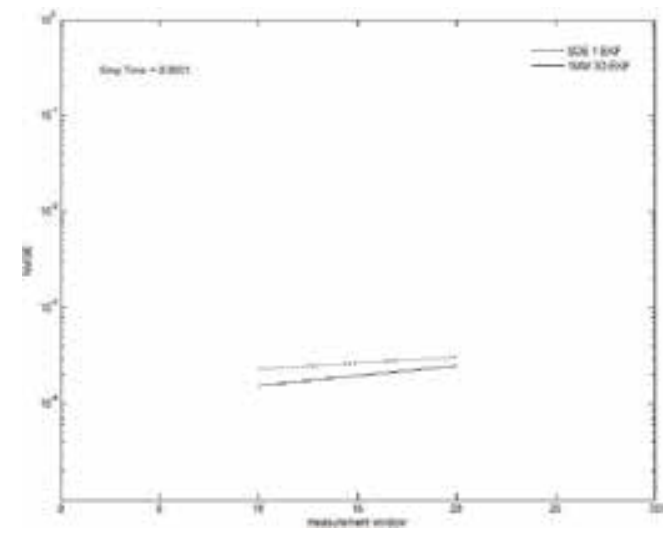

Figure 2.

Comparison between SDE method and $3 D E K F$.

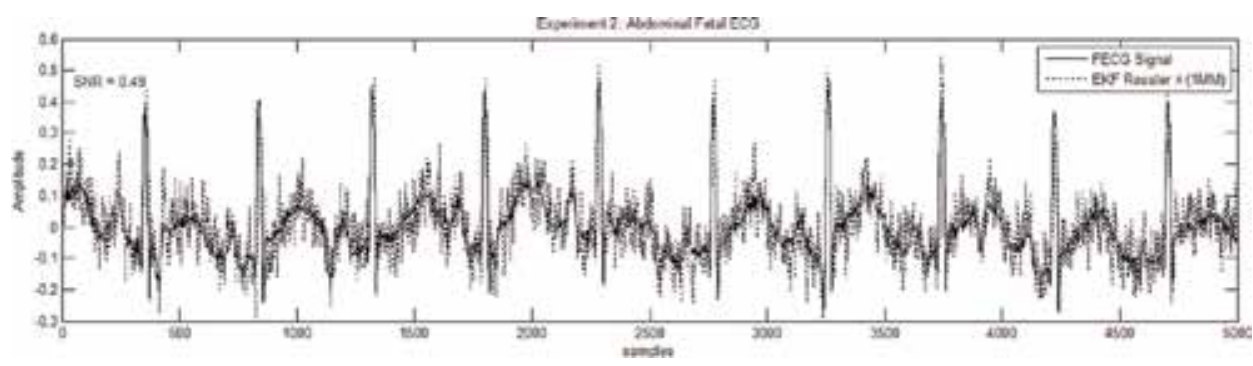

Figure 3.

Snapshot for signals in experiment two. 


\begin{tabular}{|c|c|c|c|c|}
\hline SNR & $0 \mathrm{~dB}$ & $-3 \mathrm{~dB}$ & $-10 \mathrm{~dB}$ & Processing time \\
\hline \multicolumn{5}{|c|}{ SKF $Q=0.04$ (with matrix from SIT) } \\
\hline $1 \mathrm{MM}$ & 0.0025 & 0.0037 & 0.0078 & $0.43 \mathrm{~s}$ \\
\hline $2 \mathrm{MM}$ & 0.0021 & 0.0032 & 0.0065 & $0.89 \mathrm{~s}$ \\
\hline \multicolumn{5}{|c|}{ EKF Rossler $x Q=0.21$} \\
\hline $1 \mathrm{MM}$ & 0.0026 & 0.0040 & 0.0098 & $1.825 \mathrm{~s}$ \\
\hline $2 \mathrm{MM}$ & 0.0023 & 0.0036 & 0.0079 & $3.503 \mathrm{~s}$ \\
\hline \multicolumn{5}{|c|}{ EKF Lorenz $x Q=0.42$} \\
\hline $1 \mathrm{MM}$ & 0.0029 & 0.0042 & 0.010 & $1.782 \mathrm{~s}$ \\
\hline $2 \mathrm{MM}$ & 0.0023 & 0.0034 & 0.0083 & $3.59 \mathrm{~s}$ \\
\hline \multicolumn{5}{|c|}{ EKF Chua $x Q=0.075$} \\
\hline $1 \mathrm{MM}$ & 0.0034 & 0.0053 & 0.015 & $1.812 \mathrm{~s}$ \\
\hline $2 \mathrm{MM}$ & 0.0026 & 0.0042 & 0.012 & $3.61 \mathrm{~s}$ \\
\hline
\end{tabular}

Table 2.

NMSE results for experiment two.

\subsection{Experiment three: voice sounds}

For this experiment sustained vowel sounds were used. These kinds of signals are used for voice synthesis procedures [26]. Figure 4 shows the snapshot (continuous line) of the vowel sound "O" (recorded in a sustained fashion for $5 \mathrm{~s}$ at $22050 \mathrm{~Hz}$ ) and also the filtered signal (broken line) using 2MM SKF with its matrix evaluated with the SIT. Almost identical results as in the previous experiments are shown in Table 3. For this experiment none of the components from the Lorenz attractor were suitable as a model for the voice-like signals.

\subsection{Experiment four: seismic signals}

For this experiment a MATLAB simulator based on the seismic models of [29] was used. For an SNR $=-3 \mathrm{~dB}$, Figure 5 shows the seismic signal and its filtered version using $2 \mathrm{MM}$ EKF with Rossler $\mathrm{x}$-component as a model. Full results are presented in Table 4. For the seismic signals, it was not possible to obtain an adequate linearization matrix, and so the SKF was not applied for this scenario.

\subsection{Experiment five: radio frequency interference (RFI) signals}

This experiment considers the RFI generated by computing equipment $[27,30]$ that affects the transmission of the desired information signals. For an $\mathrm{SNR}=-3 \mathrm{~dB}$,

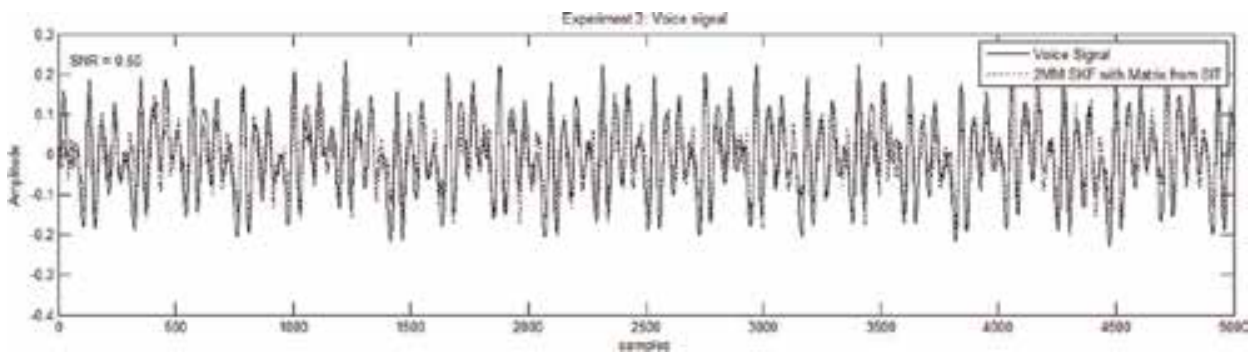

Figure 4 .

Snapshot for signals in experiment three. 
Ubiquitous Filtering for Nonlinear Problems

DOI: $h t t p: / / d x$. doi.org/10.5772/intechopen.88409

\begin{tabular}{|c|c|c|c|c|}
\hline SNR & $\mathbf{0} \mathrm{dB}$ & $-3 \mathrm{~dB}$ & $-10 \mathrm{~dB}$ & Processing time \\
\hline \multicolumn{5}{|c|}{ SKF $Q=0.0081$ (with matrix from SIT) } \\
\hline $1 \mathrm{MM}$ & 0.0025 & 0.0037 & 0.0079 & $0.47 \mathrm{~s}$ \\
\hline $2 \mathrm{MM}$ & 0.0015 & 0.0024 & 0.0053 & $0.95 \mathrm{~s}$ \\
\hline \multicolumn{5}{|c|}{ EKF Rossler $x Q=0.23$} \\
\hline $1 \mathrm{MM}$ & 0.0029 & 0.0044 & 0.0124 & $1.792 \mathrm{~s}$ \\
\hline $2 \mathrm{MM}$ & 0.0027 & 0.0039 & 0.011 & $3.611 \mathrm{~s}$ \\
\hline \multicolumn{5}{|c|}{ EKF Chua $x Q=0.76$} \\
\hline $1 \mathrm{MM}$ & 0.0031 & 0.0048 & 0.0137 & $1.81 \mathrm{~s}$ \\
\hline $2 \mathrm{MM}$ & 0.0025 & 0.0043 & 0.0130 & $3.58 \mathrm{~s}$ \\
\hline
\end{tabular}

Table 3.

NMSE results for experiment three.

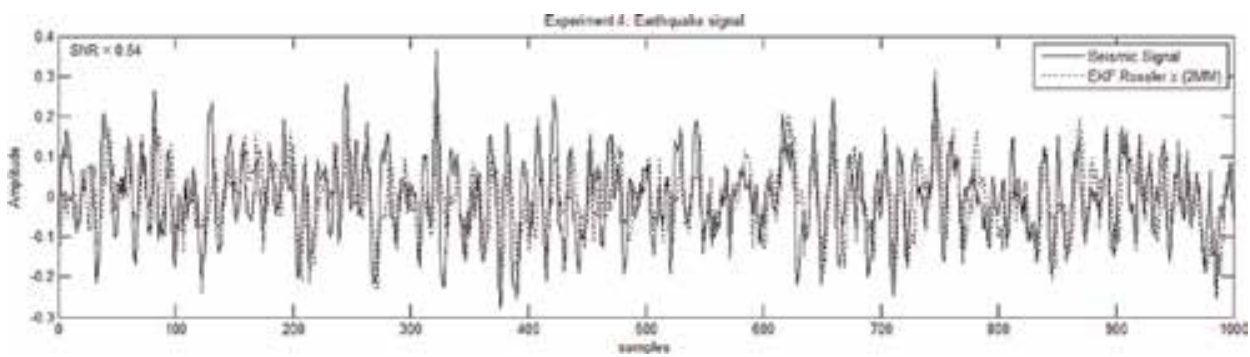

Figure 5.

Snapshot for signals in experiment four.

\begin{tabular}{|c|c|c|c|c|}
\hline SNR & $0 \mathrm{~dB}$ & $-3 \mathrm{~dB}$ & $-10 \mathrm{~dB}$ & Processing time \\
\hline \multicolumn{5}{|c|}{ EKF Rossler $x Q=0.35$} \\
\hline $1 \mathrm{MM}$ & 0.0048 & 0.0074 & 0.0178 & $1.79 \mathrm{~s}$ \\
\hline $2 \mathrm{MM}$ & 0.0047 & 0.0073 & 0.0135 & $3.53 \mathrm{~s}$ \\
\hline \multicolumn{5}{|c|}{ EKF Lorenz $x Q=0.135$} \\
\hline $1 \mathrm{MM}$ & 0.0058 & 0.0093 & 0.0245 & $1.807 \mathrm{~s}$ \\
\hline $2 \mathrm{MM}$ & 0.0054 & 0.0081 & 0.0187 & $3.62 \mathrm{~s}$ \\
\hline \multicolumn{5}{|c|}{ EKF Chua $x Q=0.135$} \\
\hline $1 \mathrm{MM}$ & 0.0057 & 0.0095 & 0.029 & $1.816 \mathrm{~s}$ \\
\hline $2 \mathrm{MM}$ & 0.0051 & 0.0084 & 0.023 & $3.65 \mathrm{~s}$ \\
\hline
\end{tabular}

Table 4.

NMSE results for experiment four.

Figure 6 shows the RFI signal and its filtered version using 1MM SKF with its matrix evaluated with the SIT. Full results are presented in Table 5.

The simulation results obtained from the linearization approach, applying SIT, are presented at Tables 2, 3, and 5. Comparative analysis of the data in the tables allows the following conclusions. All the filtering approaches presented above are rather effective, as all of them show low values of NMSE. One can notice that for the worst-case scenario $(-10 \mathrm{~dB})$, signals are visually impossible to be distinguished 


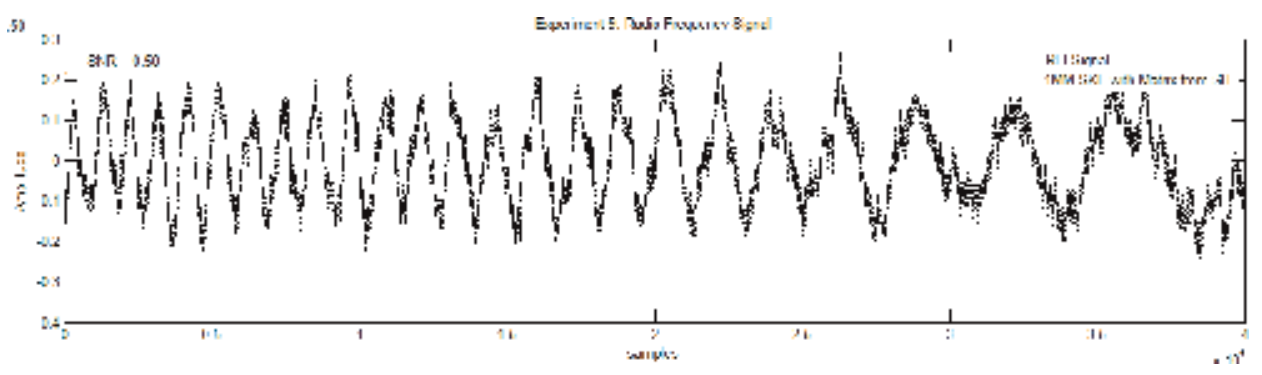

Figure 6.

Snapshot for signals in experiment five.

\begin{tabular}{|c|c|c|c|c|}
\hline SNR & $0 \mathrm{~dB}$ & $-3 \mathrm{~dB}$ & $-10 \mathrm{~dB}$ & Processing time \\
\hline \multicolumn{5}{|c|}{ SKF $Q=0.02$ (with matrix from SIT) } \\
\hline $1 \mathrm{MM}$ & 0.0018 & 0.003 & 0.0098 & $0.51 \mathrm{~s}$ \\
\hline $2 \mathrm{MM}$ & 0.0015 & 0.0025 & 0.0085 & $0.92 \mathrm{~s}$ \\
\hline \multicolumn{5}{|c|}{ EKF Rossler $x Q=0.2$} \\
\hline $1 \mathrm{MM}$ & 0.0026 & 0.005 & 0.019 & $1.872 \mathrm{~s}$ \\
\hline $2 \mathrm{MM}$ & 0.0023 & 0.0036 & 0.011 & $3.9 \mathrm{~s}$ \\
\hline \multicolumn{5}{|c|}{ EKF Lorenz $x Q=0.6$} \\
\hline $1 \mathrm{MM}$ & 0.0023 & 0.0032 & 0.04 & $1.76 \mathrm{~s}$ \\
\hline $2 \mathrm{MM}$ & 0.0016 & 0.0027 & 0.0083 & $3.81 \mathrm{~s}$ \\
\hline \multicolumn{5}{|c|}{ EKF Chua $x Q=0.4$} \\
\hline $1 \mathrm{MM}$ & 0.0034 & 0.0053 & 0.035 & $1.86 \mathrm{~s}$ \\
\hline $2 \mathrm{MM}$ & 0.0026 & 0.0042 & 0.029 & $3.77 \mathrm{~s}$ \\
\hline
\end{tabular}

Table 5 .

NMSE results for experiment five.

from noise; however, the NMSE is around 1\% for both strategies (SKF and EKF) with either $1 \mathrm{MM}$ or $2 \mathrm{MM}$.

The tables also show the average time (in seconds) required to process 5000 samples applied for statistical processing, for each filtering scenario. One has to notice that the $2 \mathrm{MM}$ approach consumes more time than the $1 \mathrm{MM}$ algorithm but (roughly speaking) no more than the double of the time required for the $1 \mathrm{MM}$ processing, as an upper bound. Second, the use of SKF is faster (almost 3 times) because there is no time consumed for the linearization process. The processing time together with the filtering complexity and fidelity might be considered as "criteria" while choosing the appropriate filtering algorithm for concrete implementations.

The SKF with the linearization approach yielded the best results; this once more confirms what was pointed above that for the processing of the quasi-linear algorithms of filtering, the influence of the spectral properties of the input data for these algorithms prevails over the influence of the "non-Gaussian" statistics of the data. The values of the NMSE, obtained by simulations, can be regarded as below or equal to the requirements for many cases of the practice, at least from the study of the corresponding references $[1,2]$ and the author's knowledge as well. Moreover, one can see that the NMSE values are rather close for all filtering scenarios, and for the 
practice, it is not so important what particular model of chaotic attractor or linearization matrix from SIT is applied!

Why it happened? This spectacular issue was briefly mentioned above, but in the following the feasible explanation is presented once more. It is worth to stress that all the chaotic attractors mentioned and applied for modeling of the real data are "generating" chaos as quasi-deterministic stochastic process Eqs. (1) and (5). Therefore all quasi-optimum filtering algorithms listed before (including EKF and its modifications) that apply chaotic modeling are working in almost "singular" regime, i.e., the shape of the a posteriori PDF is "concentrated" along the a priori PDF of the desired signal "irrespective" to the value of the SNR $[8,9]$. That is why it is possible to obtain so low values of NMSE for weak signals (SNR $<0 \mathrm{~dB}$ and down to $-10 \mathrm{~dB}$ ). Thus, for high filtering fidelity, the linear term of the Taylor expansion for the quasi-linear algorithm [4, 5, 22] significantly prevails over the terms related to the "nonlinearities" (Jacobian matrix, etc.), i.e., the linear approximation is "enough." So the influence of the nonlinear character of the ODE of the attractors on the value of the NMSE will be relatively small, which follows from the experimental data in tables. Sure, the explanation above is "qualitative" but well corresponding to the theoretic development of the quasi-optimum algorithms [4, 22].

\section{Conclusions}

In this material a rather simple and robust structure for weak signal filtering is proposed, based on the EKF algorithm and its $2 \mathrm{MM}$ modification. In addition, the linearized filtering approach is considered as well.

Based on this it is possible to suggest, for chaotic modeling of input of nonGaussian data, a "high degree of freedom" for the filtering block design depending on certain fidelity requirements and computational complexity.

Taking advantage of the quasi-linear character of the effective real-time filtering algorithms for stochastic non-Gaussian real signals, an approach using the wellknown "system identification toolbox" was proposed as well and might be selected as a reasonable compromise between computational complexity and filtering accuracy.

The experimental results show that the filtering accuracy losses for the linearization case and even for the application of the simplified SDE-1 equivalent approach are very moderate and almost negligible for practical implementations. This issue might significantly simplify the theoretical study applied for comparative selection of the filtering algorithms.

For the interested reader, it is highly recommended to consider together the material of the previous chapter "nonlinear filtering of weak chaotic signals" and the material presented above as it gives a complete "panorama" of the recommended algorithms and their real-life implementations.

All the results presented in the plots and in the tables clearly show that the implementation of the proposed strategy for solving filtering problems might be recommended for the practical scenarios. 


\section{Author details}

Valeri Kontorovich* and Fernando Ramos-Alarcon

Department of Electrical Engineering, Communications Section, CINVESTAV-IPN, Mexico

*Address all correspondence to: valeri@cinvestav.mx

\section{IntechOpen}

(C) 2019 The Author(s). Licensee IntechOpen. This chapter is distributed under the terms of the Creative Commons Attribution License (http://creativecommons.org/licenses/ by/3.0), which permits unrestricted use, distribution, and reproduction in any medium, provided the original work is properly cited. (c) BY 


\section{References}

[1] Morris AS, Langari R. Measurement and Instrumentation: Theory and Application. 2nd ed. London, UK: Academic Press Elsevier; 2016

[2] Northtrop RB. Introduction to Instrumentation and Measurements. 2nd ed. Florida, USA: CRC Press Taylor Francis; 2005

[3] Van Trees H. Detection, Estimation and Modulation Theory. New York, USA: John Wiley \& Sons; 2001

[4] Jazwinski A. Stochastic Processing and Filtering Theory. NewYork, USA: Academic; 1970

[5] Stratonovich R. Topics of the Theory of Random Noise. London, UK: Gordon Breach; 1967

[6] Wu W-R, Kundu A. Image estimation using fast modified reduced update Kalman filter. IEEE Transactions on Signal Processing. 1992;40(4): 915-926

[7] Parseh R, Kansanen K, Slock D. Distortion outage analysis for joint space-time coding and Kalman filter. IEEE Transactions on Signal Processing. 2017;65(9):2291-2305

[8] Kontorovich V, Lovtchikova Z. Nonlinear filtering of chaos for real time applications. In: Kyamakya K, Halang WA, et al., editors. Selected Topics in Nonlinear Dynamics and Theoretical Electrical Engineering, Chapter 3. Berlin Heidelberg: Springer-Verlag; 2013

[9] Kontorovich V, Lovtchikova Z, Ramos F. Nonlinear filtering of weak chaotic signals. In: Kais A, Al Naimee M, editors. Chaos Theory, Chapter 5.

London, UK: Intech; 2018

[10] Yue L, Baojun Y, et al. Ability to detect weak effective seismic signals by utilizing chaotic vibrator system.
Chinese Science Bulletin. 2006;51(24): 3010-3017

[11] Vieira M. Chaos and synchronized chaos in an earthquake model. Physical Review Letters. 1998;82(1):201-204

[12] Li Y, Yang Bj, Badal J, et al. Chaotic system detection of weak seismic signals. Geophysics Journal International. 2009;178(3):1493-1522

[13] Kolumban G, Kennedy MP, Chua LO. The role of synchronization in digital communications using chaos. I. Fundamentals of digital communications. IEEE Transactions on Circuits and Systems I: Fundamental Theory and Applications. 1997;44(10): 927-936

[14] Kaddoum G. Design and performance analysis of a multiuser OFDM based differential chaos shift keying communication system. IEEE Transactions on Communications. 2016; 64(1):249-260

[15] Thakor NV. Chaos in the heart: Signals and models. In: Proceedings of the 2nd International Conference Biomedical Engineering; May 1998; pp. 11-18

[16] Rodriguez-Bermudez G, GarciaLaencina PJ. Analysis of EEG signals using nonlinear dynamics and chaos: A review. Applied Mathematics and Information Sciences. 2015;9(5): 2309-2321

[17] Shu-Yao J, Fei Y, et al., Application of stochastic resonance technology in underwater acoustic weak signal detection. In: Conference OCEANS; April 2016; pp. 978-982

[18] Anischenko VS et al. Non-Linear Dynamics of Chaotic and Stochastic Systems. Berlin Heidelberg: SpringerVerlag; 2007 
[19] Middleton D. Introduction to the Statistical Theory of Communications. Piscataway, New Jersey: IEEE Press; 1999

[20] Primak S, Kontorovich V, Lyandres V. Stochastic Methods and their Applications to Communications: Stochastic Differential Equations Approach. Chichester West Sussex, UK: John Wiley \& Sons; 2004

[21] Pecora LM, Carroll TL. Synchronization in chaotic systems. Physical Review Letters. 1990;64(8): 821-824

[22] Pugachev V, Sinitsyn I. Stochastic Differential Systems Analysis and Filtering. Chichester West Sussex, UK: John Wiley \& Sons; 1987

[23] Kontorovich V, Lovtchikova Z. Cumulant analysis of strange attractors: Theory and applications. In:

Kyamakya K, Halang WA, et al., editors. Recent Advances in Non-Linear Dynamics and Synchronization. Berlin Heidelberg: Springer-Verlag; 2009. pp. $77-115$

[24] Nikulchev EV. Simulation of robust chaotic signal with given properties. Advanced Studies in Theoretical Physics. 2014;8(21):939-944

[25] Ljung L. System Identification: Theory for the User. New Jersey, USA: Prentice Hall; 1999

[26] Banbrook M, McLaughlin S, Mann I. Speech characterization and synthesis by nonlinear methods. IEEE Transactions on Speech and Audio Processing. 1999;7(1):1-17

[27] Arditti D. Alcocer A, Ramos F, Kontorovich V. Adaptive mitigation of platform-generated radio-frequency interference. Patents EP2838205A3, 2014 and CN104378128B. 2018
[28] PhysioNet ATM. Available from: h ttps://physionet.org/cgi-bin/atm/ATM

[29] Rofooel FR, Mobarake A, Ahmadi G. Generation of artificial earthquake records with a nonstationary Kanai-Tajimi model. Engineering Structures. 2001;23(7): 827-837

[30] Alban EX, Magaña ME, Skinner HG, Slattery KP. Statistical modelling of the interference noise generated by computing platforms. IEEE Transactions on Electromagnetic Compatibility. 2012;54(3):574-584 
Section 3

\section{Biology and Economics}





\title{
Chaos in Human Brain Phase Transition
}

\author{
Mibaile Justin, Malwe Boudoue Hubert, Gambo Betchewe, \\ Serge Yamigno Doka and Kofane Timoleon Crepin
}

\begin{abstract}
From a theoretical equation, modeling the dynamic of the time-dependent coefficients of the first and the second Karhunen-Loeve (KL) expansion of a superconducting quantum interference device (SQUID) signal, chaotic phase transition has been studied in the human brain. Through numerical investigations, the bifurcation diagram and the dynamic of Lyapunov exponent have been plotted. These diagrams reveal that throughout the variation of the control parameter here the frequency of the acoustic stimulus, the brain bifurcates from chaotic states to periodic or to quasiperiodic one. Also a chaotic phase portrait of the KL modes and its corresponding Poincaré section have been plotted. The origin of chaos in the human brain could be due to the self-organizing processes of nonequilibrium phase transition occurring in the electrochemical physiological phenomena of the complex nerve cells and neural assembly. Besides, the occurrence of chaos in the absence of stimuli has been remarked and thought to be due to the fact that an intrinsic brain could be chaotic. Moreover it has been found that the range of frequency for which the brain is forced to behave periodically could be harmful to the thinking process.
\end{abstract}

Keywords: human brain, Karhunen-Loeve coefficients, phase transition, self-organizing phenomena, chaos

\section{Introduction}

Nonlinearity is one of the most ubiquitous phenomena in nature and in our society. A system is said to be nonlinear if its output is not proportional to its input. The study of nonlinear system can be divided into six domains: fractal, pattern formation, soliton, complex systems, cellular automata, and chaos [1]. Many researches have been carried out in those six domains by scientists through the study of the dynamics of numerous systems. Generally, those systems exhibit spontaneous orders and patterns of organization governed by the self-organizing phenomena. Through the variation of an order parameter, a system can move from one state to another: this is the bifurcation phenomena, which is extremely linked to the phenomena of phase transition in some systems.

Among all the nonlinear phenomena, chaos is one of the most attractive and studied. The word "chaos" was first used in science by Li and York, who are mathematicians [2]. The Greek word chaos was originally a verb meaning to gape open and was used to refer the primeval emptiness of the universe before a thing comes into being (Encyclopedia Britannica, Vol. 5, p. 276; [3]). More simply, it means: 
- Utter disorder and confusion

- The unformed original state of the universe

For scientists, chaos is known as a nonperiodic oscillatory state, which stems from the nonlinear nature of a given system [4]. It has been observed in condensed matter physics, turbulent fluid dynamics, the Belousov-Zhabotinsky reaction in chemistry, charge density wave in low dimensional conductors, and nonlinear carrier transport in semiconductors [5]. Chaos has also been reported in economics, in social sciences, and in biological sciences.

Chaos was widely studied in human physiological systems and particularly in the human brain. The brain is the most complex and fascinating organ of the human body [6]. The human brain is constituted of many elements: neurons, neural stem cells, blood vessels, and glial cells. The neurons are about 100 billions, and for each neuron, there are more than $10^{4}$ connections to others [7]. These neuronal and nonneuronal cells are located in the cerebral cortex, outer surface of the cerebral hemispheres or the cerebrum. The brainstem lies under the cerebrum. The cerebellum is situated behind the brainstem and under the cerebrum.

The study of chaos in the brain started in the 1980s [8] when scientists observed that when rabbits inhale an odorant, their electroencephalograms (EEGs) display oscillations in the high-frequency range of $20-80 \mathrm{~Hz}$. Bressler and Freeman have named that behavior "gamma" in analogy to the high end of the X-ray spectrum [9]. The carrier wave of that odor information has exhibited an aperiodic behavior leading to the conclusion that activity of the olfactory bulb is chaotic. Therefore, chaos has been investigated in human brain elements, ranging from subcellular to cellular levels [10]. Chaos has also been studied in single neurons, in coupled neurons, in axonal membranes, and in synapses. Additionally, from the model of Hodgkin and Huxley [11, 12], chaotic dynamics of some neuron's ion conduction has been recorded.

The study of chaos in the human brain has an importance which could no longer be demonstrated. It has been reported that chaos plays an important role in cortical hormone secretion and suppression [13]. Moreover, chaotic behavior stemming from self-organization processes in the human brain could explain "randomness" in neural synchronization related to cognitive functions and consciousness and also in mental disorganization related to psychopathological phenomena such as schizophrenia [14]. In addition, it has been argued that epilepsy is an example of chaos in the human brain [15]. Besides, some researchers think about the dreaming brain as a brain in which some self-organizing processes occur and exhibit chaos-like stochastic properties that are highly sensitive to internal influences [16].

In this paper, we deal with a synergetic view of the human brain. In this view the brain acts by means of self-organization processes through which nonequilibrium phase transitions occur. By means of 37 superconducting quantum interference devices (SQUID), the values of the magnetic field generated by the intracellular dendritic current of the brain of a subject exposed to acoustic stimuli have been recorded [17]. From that experiment, Fuchs and co-workers studied the human brain phase transition and chaos $[18,19]$. The aim of this paper is to theoretically study chaos from equations derived by Jirsa and co-workers [20] based on the works of Kelso and co-workers. To reach such a goal, we outline our work as follows: Section 2 deals with the presentation of the equation model and its linear stability, Section 3 describes the numerical studies of chaos, and the paper ends with concluding remarks. 


\section{Presentation of the model and its linear stability}

\subsection{Origin of the model}

Here we will describe how the equation modeling the brain phase transition was derived. This equation is based on the experimental study carried out by Kelso and co-workers [17]. In that study, a set of 37 SQUID collect the values of the magnetic field generated by the intracellular dendritic current of the brain of a subject exposed to periodic acoustic stimuli. This noninvasive brain exploration was rendered possible because the skull and the scalp are permeable to the magnetic field generated inside the brain. The stimuli served as control parameter, and the subject was invited to press a button in two successive tones. The spatiotemporal behavior of the brain signals was known after the application of a Karhunen-Loeve expansion to the magnetic field collected by the SQUID array. Based on the observations of results of this experiment, Jirsa and co-workers have introduced a mathematical model which mimics the brain behavior [20]. That model was made of two nonlinearly coupled oscillators describing the coefficients of the first KL modes that are driven by the acoustic stimuli. That model provided a mathematical description of the switching from the first KL mode that oscillated at the stimuli frequency to the second KL mode that oscillated at twice the stimuli frequency [20].

The following mathematical model is a set of differential equations [20]:

$$
\begin{gathered}
\ddot{x}+\left(\gamma_{1}+A_{1} x^{2}+B_{1} y^{2}\right) \dot{x}+\omega_{01}^{2}\left(1+\epsilon_{1} \sin (2 \Omega t)\right) x+C_{1} y^{2} \sin (\Omega t)=0, \\
\ddot{y}+\left(\gamma_{2}+A_{2} y^{2}+B_{2} x^{2}\right) \dot{y}+\omega_{02}^{2}\left(1+\epsilon_{2} \sin (2 \Omega t)\right) y+D \dot{x}=0 .
\end{gathered}
$$

where the parameters $\mathrm{x}$ and $\mathrm{y}$ represent the time-dependent coefficients of the first and the second Karhunen-Loeve expansions of SQUID signals, respectively. Also, the coefficients $\gamma_{i}, A_{i}, B_{i}, \omega_{0 i},(i=1,2), C_{1}$, and $D$ are adjustable but then fixable parameters [20]. The control parameter of the system here is $\Omega$ which is the frequency of the acoustic signal used to stimulate the subject. Here we neglect the random processes.

\subsection{Study of the linear stability of the model}

In others to study easily Eqs. (1) and (2), we set

$$
\begin{aligned}
& \dot{x}=u, \\
& \dot{y}=v, \\
& \theta=\Omega t .
\end{aligned}
$$

This leads to

$$
\begin{gathered}
\dot{x}=u, \\
\dot{u}+\left(\gamma_{1}+A_{1} x^{2}+B_{1} y^{2}\right) u+\omega_{01}^{2}\left(1+\epsilon_{1} \sin (2 \Omega t)\right) x+C_{1} y^{2} \sin (\theta)=0, \\
\dot{y}=v, \\
\dot{v}+\left(\gamma_{2}+A_{2} y^{2}+B_{2} x^{2}\right) v+\omega_{02}^{2}\left(1+\epsilon_{2} \sin (2 \theta)\right) y+D u=0 \\
\dot{\theta}=\Omega .
\end{gathered}
$$


For $\Omega=0$, Eqs. (6) $-(10)$ possesses a circle of fixed points $\mathrm{X}(0,0,0,0, \theta)$ with $\theta$ in $\mathrm{R} \bmod 2 \pi$. This circle of fixed points becomes a limit cycle of Eqs. (1) and (2). The corresponding Jacobian matrix for $\Omega=0$ is then given as

$$
J=\left[\begin{array}{ccccc}
0 & 1 & 0 & 0 & 0 \\
-\omega_{01}^{2} & -\gamma_{1} & 0 & 0 & 0 \\
0 & 0 & 0 & 1 & 0 \\
0 & -D_{2} & -\omega_{02}^{2} & -\gamma_{2} & 0 \\
0 & 0 & 0 & 0 & 0
\end{array}\right]
$$

The associate Eigenvalues are

$$
\left[\begin{array}{c}
0 \\
-1 / 2 \gamma_{2}+1 / 2 \sqrt{\gamma_{2}^{2}-4 \omega_{02}^{2}} \\
-1 / 2 \gamma_{2}-1 / 2 \sqrt{\gamma_{2}^{2}-4 \omega_{02}^{2}} \\
-1 / 2+1 / 2 \sqrt{\gamma_{1}^{2}-4 \omega_{01}^{2}} \\
-1 / 2-1 / 2 \sqrt{\gamma_{1}^{2}-4 \omega_{01}^{2}}
\end{array}\right]
$$

When $\Omega \neq 0$, the corresponding Jacobian matrix is then given as

$$
J=\left[\begin{array}{ccccc}
0 & 1 & 0 & 0 & 0 \\
-\omega_{01}^{2}\left(1+\varepsilon_{1} \sin (2 \Omega)\right) & -\gamma_{1} & 0 & 0 & 0 \\
0 & 0 & 0 & 1 & 0 \\
0 & -D_{2} & -\omega_{02}^{2}\left(1+\varepsilon_{2} \sin (2 \Omega)\right) & -\gamma_{2} & 0 \\
0 & 0 & 0 & 0 & 0
\end{array}\right] .
$$

The associate Eigenvalues are

$$
\left[\begin{array}{c}
0 \\
-1 / 2 \gamma_{2}+1 / 2 \sqrt{\gamma_{2}^{2}-4 \omega_{02}^{2}-4 \omega_{02}^{2} \varepsilon_{2} \sin (2 \Omega)} \\
-1 / 2 \gamma_{2}-1 / 2 \sqrt{\gamma_{2}^{2}-4 \omega_{02}^{2}-4 \omega_{02}^{2} \varepsilon_{2} \sin (2 \Omega)} \\
-1 / 2+1 / 2 \sqrt{\gamma_{1}^{2}-4 \omega_{01}^{2}-4 \omega_{01}^{2} \varepsilon_{1} \sin (2 \Omega)} \\
-1 / 2-1 / 2 \sqrt{\gamma_{1}^{2}-4 \omega_{01}^{2} 4 \omega_{01}^{2} \varepsilon_{1} \sin (2 \Omega)}
\end{array}\right]
$$

It is clear that depending on the values of the parameters $\gamma_{i}$ and $\omega_{i}(i=1,2)$, the fixed points could either be stable or unstable.

\section{Chaos: numerical simulation and discussions}

For numerical investigations of chaos, we consider a subject with the following constant parameters: $\gamma_{1}=0.1, \gamma_{2}=0.1, A_{1}=3, A_{2}=9.8, B_{1}=2.2, \epsilon_{1}=17.09$, $\epsilon_{2}=2.99, \omega_{1}=2 \pi, \omega_{2}=2 \pi, D_{2}=10, B_{2}=2.2$ and $C_{1}$.

The numerical simulations are carried out via Runge-Kutta algorithm. The dynamic of the maximum of the Lyapunov exponent, (i.e., the greatest Lyapunov exponent among the five related to the degree of freedom of the system), is given by Figure 1. It has been plotted by following the algorithm of Wolf et al. [4] which is an appropriate tool for numerical calculation of Lyapunov exponents. 
It has been obtained through the variation of the order parameter $\Omega$ for the values of $0-100$.

The bifurcation diagram associated with this system is depicted at Figure $\mathbf{2}$ for the same range of parameter values as for the Lyapunov exponent. This diagram has been obtained by means of Runge-Kutta algorithm with a finish value of time of 90 , a transient of 30, and a time step of 1 .

The analysis of these two diagrams reveals the fact that depending on parameter range, the system is subject to various behaviors. For example, for $\Omega \epsilon[0,0.1]$ the system behaves chaotically, while for $\Omega \epsilon[0.1,0.12]$ it behaves periodically. Throughout the variation of the control parameter, the system bifurcates from chaotic states to periodic or to quasiperiodic ones. This means that the brain

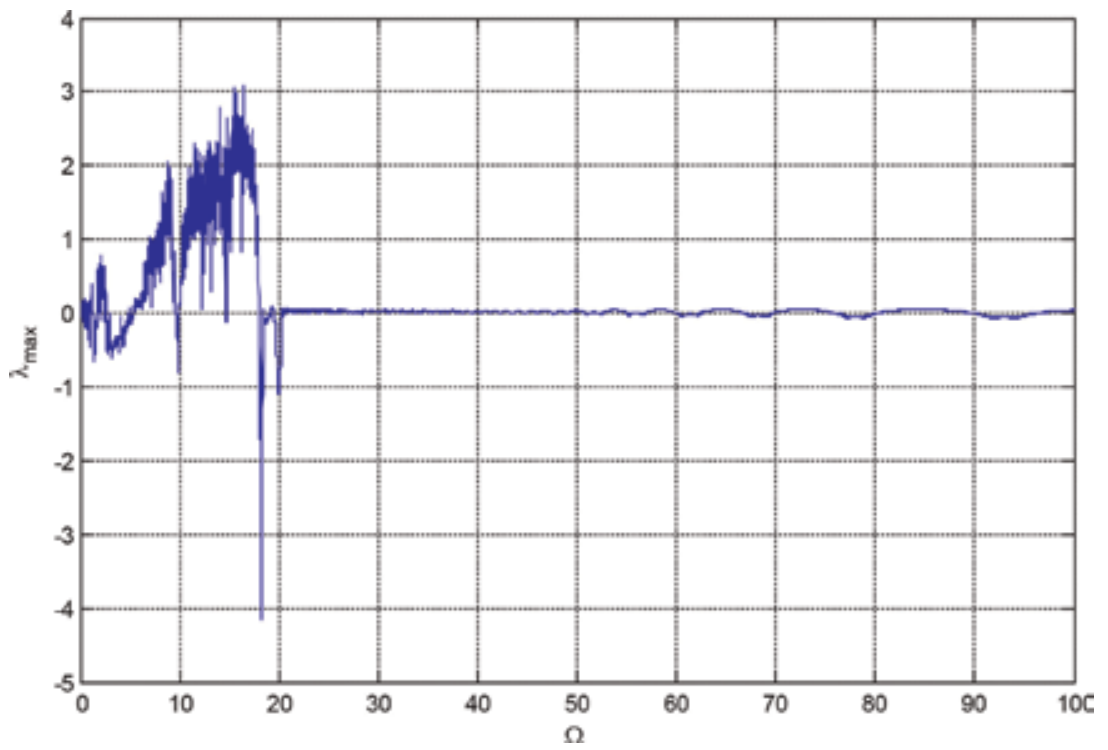

Figure 1.

Dynamics of the maximum of the Lyapunov exponents obtained through the variation of the stimuli frequency

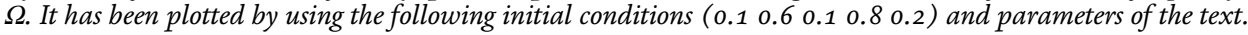

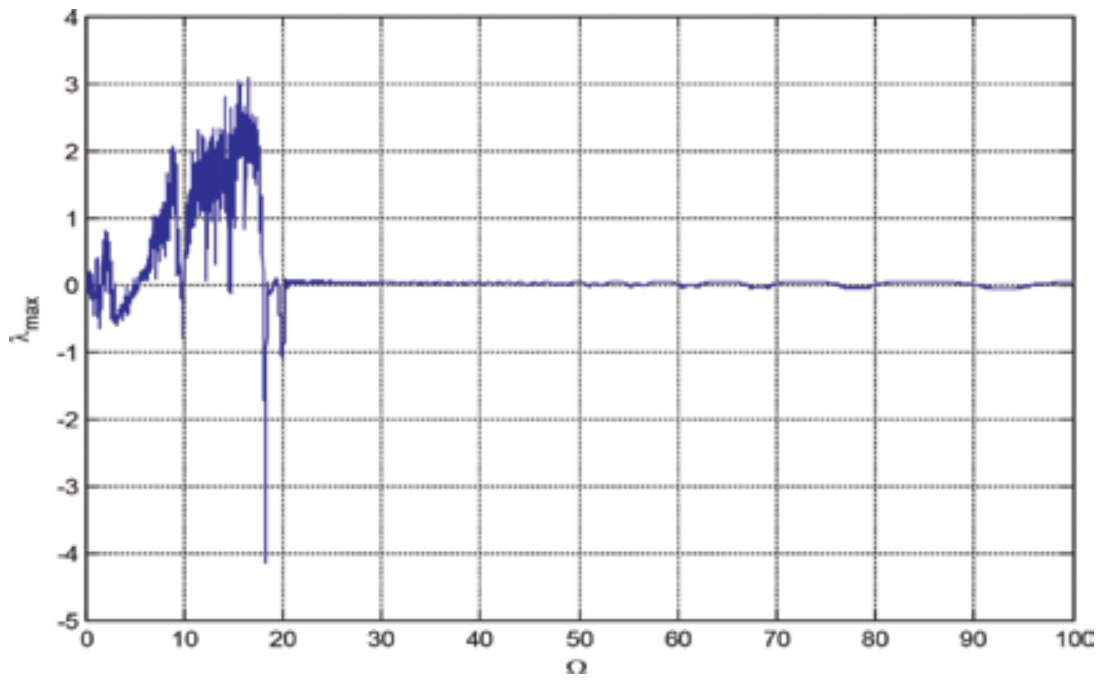

Figure 2.

Bifurcation diagram corresponding to the maximum of the first KL coefficient (i.e., $x$ ). It has been obtained for the same range of parameters as for the Lyapunov exponent. 
experiences periodic states, quasiperiodic states, intermittencies, and chaos. The fact that chaos occurs even for $\Omega=0$ could explain the chaotic behavior of an intrinsic human brain, the dreaming brain, for example [15].

In addition to the above diagrams, we present the $2 \mathrm{D}$ phase portrait $(\mathrm{x}, \mathrm{y})$ of the KL given by Figure 3 for $\Omega=15$ with the parameters of Figure 2 .

The topological mixed nature of this phase portrait reveals its chaotic nature. Figure 4 represents the Poincaré section corresponding to the 2D phase portrait of Figure 3. It has been obtained by cutting the $3 \mathrm{D}$ phase space $(\mathrm{x}, \mathrm{u}, \mathrm{y})$ at $\mathrm{u}=5$.

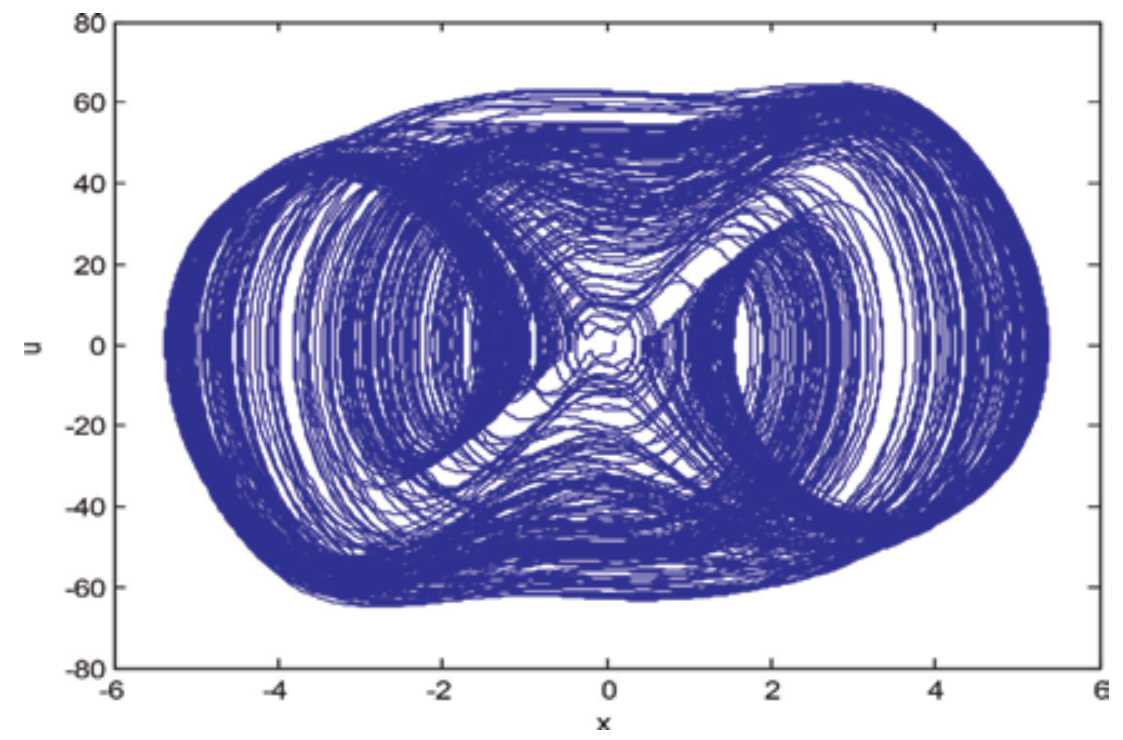

Figure 3.

$2 D$ phase portrait $(x, y)$ of the KL coefficients obtained for $\Omega=15$.

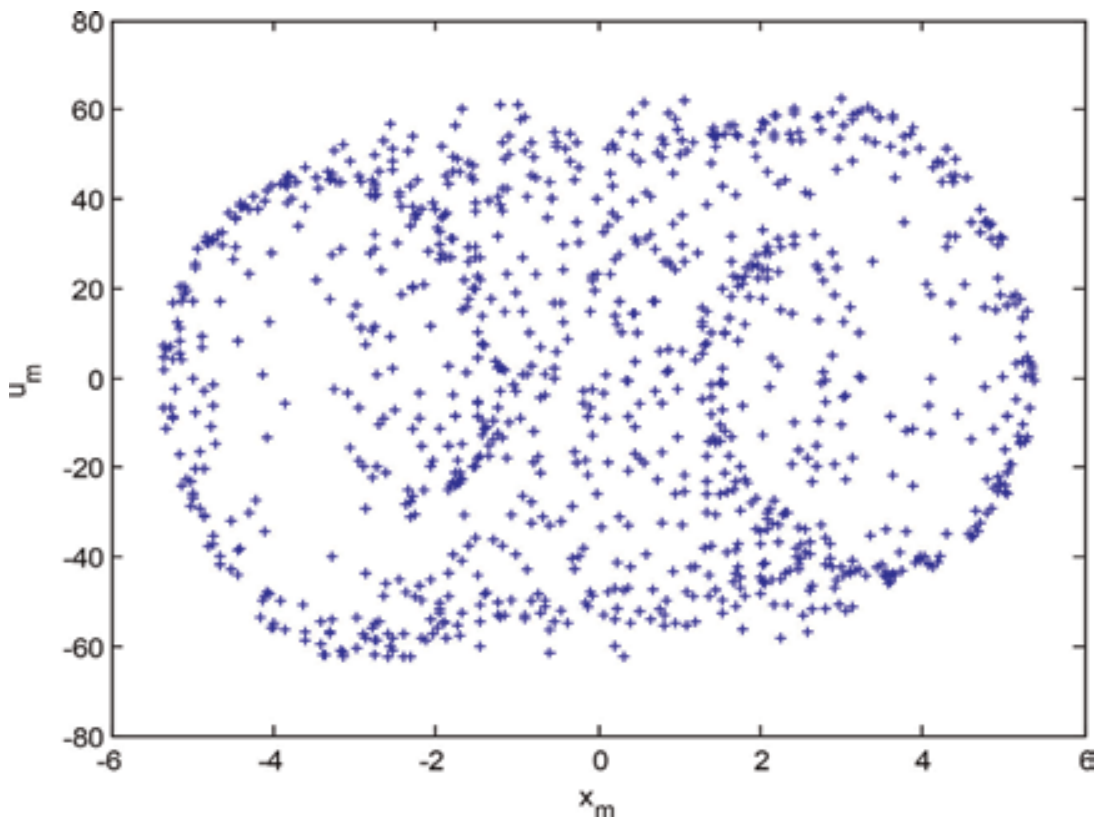

Figure 4 .

$2 D$ Poincare section of the previous phase portrait obtained by cutting the $3 D$ phase space $(x, u, y)$ at $u=5$. 


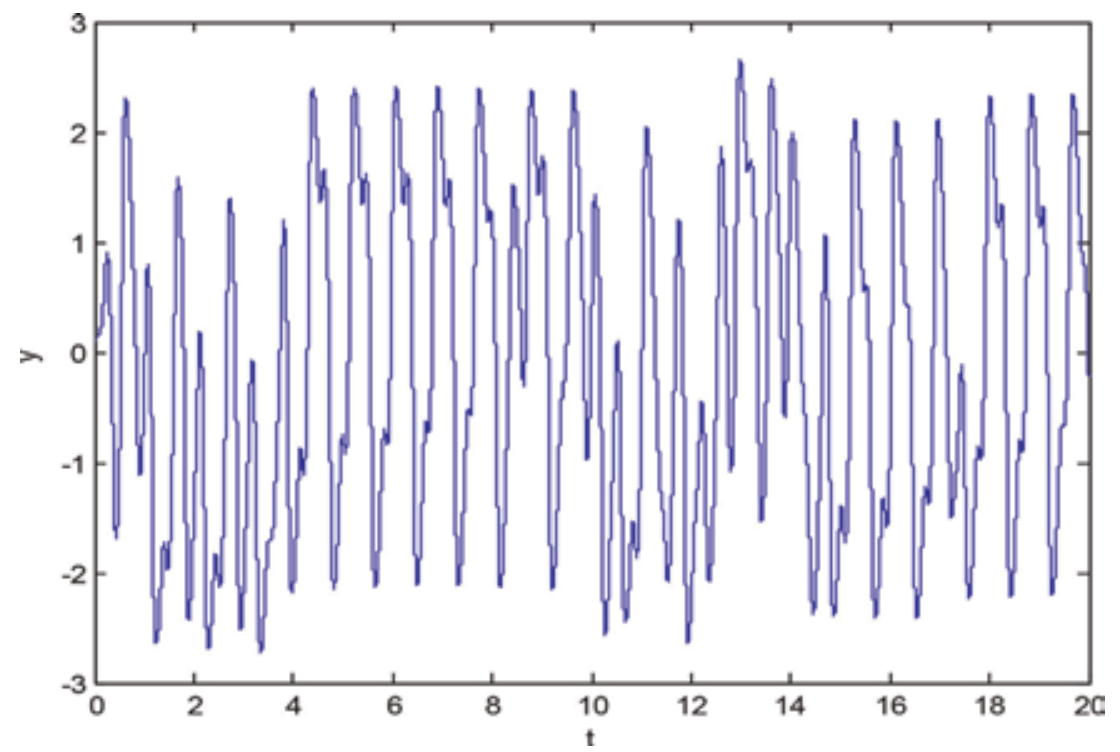

Figure 5 .

Chatic wave form of the second KL coefficients obtained for $\Omega=15$.

Its non-ordered nature confirms that the previous phase portrait is really chaotic.

The origin of chaos in the human brain could be due to the self-organizing process of nonequilibrium phase transition occurring in the electrochemical physiological phenomena of the complex nerve cells and neural assembly.

The chaotic wave form of the second KL coefficients obtained for $\Omega=15$ is represented by Figure 5. The wave does not conserve its form throughout the time meaning that there is a loss of memory while the system evolves.

\section{Conclusion}

In this study, we dealt with chaos in the human brain. By means of numerical investigations through an equation modeling a human brain phase transition, some interesting results have been found. The linear stability of the equation model has been done: the system possesses one fixed point which could either be stable or unstable depending on the values of some parameters. Additionally, the bifurcation diagram and the dynamic of the Lyapunov exponent have been plotted. The examination of those diagrams reveals the fact that the brain can undergo periodic phase transition, quasiperiodic phase transition, and chaotic phase transition. Chaos may be due to a nonequilibrium phase transition, happening even when there are no stimuli, meaning that an intrinsic brain could be a chaotic one. The Lyapunov exponent and the unrepeatable waveform of the second KL coefficients underline the loss of memory by the system revealing that nonequilibrium phase transition of the brain is not reversible. Therefore the electrochemical physiological phenomenon of the brain is a dissipative one.

There are two opinions about human brain chaos. Chaos was firstly discovered at a neural level and has led researchers to a thing about chaos as a source of some diseases: schizophrenia, insomnia, and epilepsy [21]. The studies of chaos on the macroscopic level by other researchers reveal the fact that chaos is related to thinking and only the brain of falling sickness is periodic [22]. Also chaos helps the brain to quickly recognized previously learned patterns and behaviors [23]. From 
these above ideals, the importance of chaos in the brain is no longer to be demonstrated.

In this study, we have realized that there are some frequencies for which the brain behaves periodically and others for which its behavior is chaotic. This means that some acoustic stimulus at some frequencies could alter the brain functioning. For example, for $>20$, the brain is forced to behave periodically and could have some harmful feedback on the thinking process.

Also, this study can help for the development of neuromorphic and artificial neural network architectures since such technologies are based on human brain functioning. The understanding of chaos in human brain electromagnetic activity could help for deeper understanding of the role of chaos in the brain. This could be the object of future work.

\section{Conflict of interest}

The authors declare there is no conflict of interest.

\section{Author details}

Mibaile Justin $^{1 *}$, Malwe Boudoue Hubert ${ }^{2}$, Gambo Betchewe ${ }^{2}$, Serge Yamigno Doka ${ }^{3}$ and Kofane Timoleon Crepin ${ }^{4}$

1 Higher Teachers' Training College of Maroua, The University of Maroua, Cameroon

2 Department of Physics, Faculty of Science, The University of Maroua, Cameroon

3 Department of Physics, Faculty of Science, The University of Ngaoundere, Cameroon

4 Department of Physics, Faculty of Science, The University of Yaounde I, Cameroon

*Address all correspondence to: thejust7@yahoo.fr

\section{IntechOpen}

(C) 2019 The Author(s). Licensee IntechOpen. This chapter is distributed under the terms of the Creative Commons Attribution License (http://creativecommons.org/licenses/ by/3.0), which permits unrestricted use, distribution, and reproduction in any medium, provided the original work is properly cited. (cc) BY 


\section{References}

[1] Lam L. Introduction to Nonlinear Physics. New York: Springer-Verlag; 1997. $417 \mathrm{p}$

[2] Li TY, York JA. Period three implies chaos. The American Mathematical Monthly. 1975;82:985-992

[3] Moon FC. Chaotic Vibration-an Introduction for Applied Scientists and Engineers. New York: John Wiley and Sons; 1987. 309p

[4] Wolf A, Swift JB, Swinney HL, Vastano JA. Determining Lyapunov exponents from a time series. Physica D: Nonlinear Phenomena. 1985;16:285-317

[5] Mibaile J, Gambo B, Serge YD, Yves JE, Kofane TC. Chaos in semiconductor band-trap impact ionization. Current Applied Physics. 2013;13:1209-1212

[6] Forstmann BU, Wagenmakers EJ. An Introduction to Model-Based Cognitive Neuroscience. New York/Heidelberg/ Dordrecht/London: Springer; 2015. 354p

[7] Hermann H. Principles of Brain Functioning. Berlin/Heidelberg: Springer-Verlag; 1996. 350p

[8] Freeman WJ. Neurodynamics: An Exploration in Mesoscopic Brain Dynamics. Perspectives in Neural Coding. New York: Springer; 2000. $398 \mathrm{p}$

[9] Bressler SL, Freeman WJ. Frequency analysis of olfactory EEG in cat, rabbit and rat. Electroencephalography and Clinical Neurophysiology. 1980;50: 19-24

[10] Korn H, Philippe F. Is there chaos in the brain? II. Experimental evidence and related models. Comptes Rendus Biologies. 2003;326:787-840

[11] Jack JJB, Noble D, Tsien RW. Electric Current Flow in Excitable Cells. Oxford: Clarendon Press; 1983. 534p
[12] Koch C, Bernander O. Methods in Neuronal Modeling. Cambridge, MA, USA: MIT Press; 1989. pp. 171-194

[13] Aristides D, Athanassios I, Panos M. Nonlinear dynamics in Clinical Pharmacology: The paradigm of cortisol secretion and suppression. British Journal of Clinical Pharmacology. 2002; 54:21-29

[14] Petr B. Chaos, cognition and disordered brain. Activitas Nervosa Superior. 2008;50:114-117

[15] Iasemidis LD, Sackellares JC. Measuring chaos in the human brain. The Neuroscientist. 1996;2:118-126

[16] David K, Allan C, Stanley K. Dreaming as a function of chaos-like stochastic processes in the selforganizing brain. Nonlinear dynamics, psychology, and life sciences. 2002;6: 311-322

[17] Kelso J, Bressler SL, Buchanan S, DeGuzman GC, Ding M, Fuchs A, et al. A phase transition in human brain and behavior. Physics Letters A. 1992;169: 134-144

[18] Fuchs A, Kelso JAS, Herman H. Phase transitions in the human brain: Spatial mode dynamics. International Journal of Bifurcation and Chaos. 1992; 2:917-939

[19] Kelso JAS, Fuchs A. Self-organizing dynamics of the human brain: Critical instabilities and SiI'nikov chaos. Chaos. 1995;5:64-69

[20] Jirsa VK, Friedrich R, Hakcn H, Kelso JAS. A theoretical model of phase transitions in the human brain. Biological Cybernetics. 1994;71:27-35

[21] Guevara MR, Glass L, Mackey MC, Shrier A. Chaos in neurobiology. IEEE Transactions on Systems, Man, and Cybernetics. 1983;SMC-13:790-798 
[22] Yi-Fang C. Neural synergetics, Lorenz model of brain, soliton-chaos double solutions and physical neurobiology. NeuroQuantology. 2013; 11:56-62

[23] Freeman WJ, Yao Y. Central pattern generating and recognizing in olfactory bulb. Neural Networks. 1990;3:153-170 


\title{
Global Indeterminacy and Invariant Manifolds Near Homoclinic Orbit to a Real Saddle
}

\author{
Beatrice Venturi
}

\begin{abstract}
In this paper we investigate the dynamic properties of the Romer model. We determine the whole set of conditions which lead to global indeterminacy and the existence of a homoclinic orbit that converges in both forward and backward time to a real saddle equilibrium point. The dynamics near this homoclinic orbit have been investigated. The economic implications are discussed in the conclusions.
\end{abstract}

Keywords: externality, endogenous growth model, homoclinic orbits to real saddle, stable cycle, global indeterminacy

\section{Introduction}

In this note we prove the existence of a homoclinic orbit in an extension (see [1]) of well-known endogenous growth two-sector technological change Romer model [2], introduced in [3]. It represents the first attempt to make formal a model of endogenous growth, through research and development (R\&D) activities.

In this model, the knowledge is composed of two components, human capital, which defines the specific knowledge of every person, and the so-called technology, that, in general, is available for everybody.

The first component ascribes the rivalry feature to its employment because it is incorporated in the physical person. Indeed, a human resource used by a firm cannot be used by another firm. The second component of knowledge ascribes the feature of non-rivalry good, because it can be used by different firms in the same time. The consequence of the human capital rivalry, who invests in human capital accumulation, receives the profit of this accumulation too, while the non-rivalry feature implies the spillover effects diffusion, that is, the inventor of a new technology will not be the only beneficiary of the positive effects related to this discovery. It is impossible to him to take total possession of his fruits. This fact implies the development of an externality that, in turn, reduces the single person efforts to improve the productive technology under the level that should be socially advisable.

An externality is an economic action effect that involves another subject, not directly implicated in this action (change, production, or consumption action).

The market equilibrium in the presence of externalities is not optimal, because expenses and private utilities do not coincide to expenses and social utilities (e.g., pollution). Therefore, the external effects, positive and negative, are not, 
respectively, remunerated or compensated. Both the human capital and the technology are fruits of conscious human choices.

Moreover the research activity is intensive in the use of human capital and technology too, without physical capital $\mathrm{K}$ and skilled labor is employed in research.

In several papers the question of the uniqueness of the equilibrium trajectory has been studied for this model. Benhabib, Perli, and Xie (BPX), in [3], used numerically analysis for proving the existence of stable periodic solutions in a generalized version of the Romer model in which they consider the complementarity between different intermediary capital goods.

Finally, [2] extends BPX model by tightening the parameter restrictions necessary to obtain an interior steady state and studies the stability of the steady state in BPX model without unskilled labor.

Following [4], we consider the three-dimensional reduced version of the model, obtained by a standard change of variables, related with the growth rate of the fundamental variables.

By using the method of undetermined coefficients (see [5]), we are able to prove the existence of a homoclinic orbit that converges both forward and backward to the unique equilibrium point whose linearization matrix admits two positive and one negative real eigenvalues. The stable and unstable manifolds are locally governed by real eigenvalues. The concept of homoclinic bifurcations is very important from a dynamic point of view. Such phenomena causes global rearrangements in phase space, including changes to basins of attractions and generation of chaotic dynamics [6].

We show that such a homoclinic orbit gives rise to global indeterminacy in a parameter set commonly investigated by means of the instruments of the local analysis.

The paper develops as follows. In the second section, we analyze the optimal control model, and we introduce the equivalent three-dimensional continuous time abstract stationary system. The third section is devoted to the steady-state analysis of the model in reduced form. In the fourth section, we apply the procedure developed by [5], and we show that a homoclinic loop emerges as a solution trajectory. In the last section, we consider a homoclinic bifurcation of dimension one. The economic implications are discussed in the conclusions.

\section{The model}

We consider now the three-dimensional reduced version of the Romer model. In the original optimal control model, the state variables are $\mathrm{k}$, the physical capital; A, the level of knowledge currently available [1, 2]; and

$$
\dot{k}=Y \div C=\eta^{\gamma} k^{\gamma} A^{\xi-\gamma} h^{\alpha} L^{\beta}
$$

$\xi \geq 1$ is the degree of complementarity, $\gamma$ is a positive externality parameter in the production of physical capital, $\beta$ is the share of capital, and $\alpha$ is defined by the following relationship $\alpha=1-\gamma-\beta$. The control variables are $h$ the human capital, the skilled labor employed in the final sector Y, $C$ is the consumption, $\rho$ is a positive discount factor, and $\sigma$ is the inverse of the intertemporal elasticity of substitution. The only consumption good $C$ is measured in units of the final output $Y$. The final output is produced with two capital goods, the physical capital $k$ and human capital $h$. 
We consider the following substitution:

$$
r=k A^{\frac{\alpha+\beta+\gamma}{\alpha+\beta}} ; h=h ; q=\frac{c}{k}
$$

where $r$ is the interest rate. We set $\Lambda=\alpha \xi /(\gamma-\xi)$

The deterministic reduced form of this model is given by

$$
\left\{\begin{array}{c}
(r / r)=(1 /(1-\alpha))\left((\xi-1+\beta) \delta(1-h)-\beta\left(\left(\xi /\left(\gamma^{2}\right)\right) r-q\right)-\alpha(r-(\delta / \Lambda) h)\right) \\
(h / h)=(1 /(1-\alpha))\left((\xi-1-\gamma) \delta(1-h)-\gamma\left(\left(\xi /\left(\gamma^{2}\right)\right) r-q\right)-\alpha(r-(\delta / \Lambda) h)\right)(\mathrm{S}) \\
(q / q)=((r-\rho) / \sigma)-\left(\xi /\left(\gamma^{2}\right)\right) r+q
\end{array}\right.
$$

The set of parameters $\omega \equiv(\beta, \xi, \alpha, \delta, \gamma, \rho, \sigma)$ lives inside a significant economic parameter set $\Omega \equiv\left\{(0,1) \times \mathbb{R}_{++} \times \mathbb{R} \times \mathbb{R} \times \mathbb{R} \times \mathbb{R}_{++} \times \mathbb{R}_{++}-\{1\}\right.$.

\section{Steady-states analysis}

A stationary (equilibrium) point $\mathrm{P}^{*}=P(r *, h *, q *)$ of the system (S) is any solution of the following system:

$$
\left\{\begin{array}{r}
1 /(1-\alpha)(\xi-1+\beta) \delta(1-\mathrm{h})-\beta\left(\left(\xi /\left(\gamma^{2}\right) \mathrm{r}-\mathrm{q}\right)-\alpha(\mathrm{r}-(\delta / \Lambda) \mathrm{h})=0\right. \\
1 /(1-\alpha)(\xi-1-\gamma) \delta(1-\mathrm{h})-\gamma\left(\left(\xi /\left(\gamma^{2}\right)\right) \mathrm{r}-\mathrm{q}\right)-\alpha(\mathrm{r}-(\delta / \Lambda) \mathrm{h})=0 \\
(\mathrm{r}-\rho) / \sigma)-\left(\xi /\left(\gamma^{2}\right)\right) \mathrm{r}+\mathrm{q}=0
\end{array}\right.
$$

We get only one admissible steady state:

$$
\left\{\begin{array}{r}
h *=\left(\frac{\Lambda}{\delta}\right)\left(\frac{((\sigma(\xi-\gamma)-(\xi-1))-\rho(1-\gamma))}{(\Lambda(\sigma(\xi-\gamma)-(\xi-1))-(1-\gamma)}\right) \\
r *=\left(\frac{1}{1-\left(\frac{1}{\sigma}\right)}\right)\left(\left(\frac{\delta}{\Lambda}\right) h *-\delta(1-h *)-\left(\frac{\rho}{\sigma}\right)\right) \sigma \neq 1 \\
r *=\left(\frac{1}{(1-\gamma)}\right)\left((\xi-1-\gamma) * \delta(1-h *)+\left(\frac{\delta}{\Lambda}\right) h *-\left(\frac{\rho}{\sigma}\right)\right) \sigma=1 \\
q *=\left(\left(\xi /\left(\gamma^{2}\right)\right)-(1 / \sigma) r *-(\rho / \sigma)\right.
\end{array}\right.
$$

We denote $J=J(P *)$ the Jacobian matrix $\mathrm{J}$ of the system (S) evaluated in the equilibrium point $P^{*}$, given by

$$
J(P *)=\left(\begin{array}{ccc}
\left(\frac{r *}{(1-\alpha)}\right) \alpha+\beta\left(\frac{\xi}{\gamma 2}\right) & \left(\frac{\delta}{(1-\alpha)}\right) r *((\alpha / \Lambda)-(\xi-1+\beta)) & \frac{\beta}{(1-\alpha)} r * \\
\left(\frac{h *}{(1-\alpha)}\right)\left(\frac{\xi}{\gamma}-1\right) & \left(\frac{\delta}{(1-\alpha)}\right) h *((1 / \Lambda)-(\xi-1-\gamma)) & \left(\frac{\gamma}{(1-\alpha)}\right) h * \\
q *(1 / \sigma)-\frac{\xi}{\gamma 2} & 0 & q *
\end{array}\right)
$$


Lemma: We consider the following two subsets of the parameters space $\Omega$ :

$$
\begin{array}{r}
\boldsymbol{\Omega}_{1}=\left\{\begin{array}{r}
\omega \in \boldsymbol{\Omega}: \boldsymbol{\delta} \boldsymbol{H}(\boldsymbol{\sigma}(\boldsymbol{\xi}-\boldsymbol{\gamma})-(\boldsymbol{\xi}-\mathbf{1}))-\rho(1-\gamma)<0 \rho>(\delta / \Lambda) H \\
((1-\sigma)(\xi-\gamma) \delta H-(1+\boldsymbol{\Lambda}))-\rho(1-\gamma))>0
\end{array}\right. \\
\boldsymbol{\Omega}_{2}=\left\{\begin{array}{r}
\omega \in \boldsymbol{\Omega}: \boldsymbol{\delta} \boldsymbol{H}(\boldsymbol{\sigma}(\boldsymbol{\xi}-\boldsymbol{\gamma})-(\xi-1))-\rho(1-\gamma))<0 \rho<(\delta / \Lambda) H \\
((1-\sigma)(\xi-\gamma) \delta H-(1+\Lambda))-\rho(1-\gamma))<0
\end{array}\right.
\end{array}
$$

(a) If the model parameters belong to set $\boldsymbol{\Omega}_{\mathbf{2}}$, then as shown in [1], the unique interior steady state is determinate.

(b) If the model parameters belong to set $\boldsymbol{\Omega}_{1}$, then as shown in [1], the unique interior steady state is indeterminate or unstable.

\section{The existence of a saddle with three purely real eigenvalue state analyses}

We are interested in the special case in which $\mathrm{J}=\mathrm{J}\left(P^{*}\right)$ has three real eigenvalues. To this end, we analyze the dynamics of the model around the equilibrium point: $P^{*}$ in $\boldsymbol{\Omega}^{\mathbf{1}}$.

Lemma: We consider the following subsets of the parameters space $\Omega_{1}$ :

$\Omega_{\mathrm{IR}}=\left\{\omega \in \Omega_{1}: \mathrm{J}\left(\mathrm{P}^{*}\right)\right.$ possesses real eigenvalues $\}$.

Let $\omega \in \Omega_{\mathrm{IR}}$ be. Then $\mathrm{J}\left(\mathrm{P}^{*}\right)$ has one positive and two negative purely real eigenvalues.

Proof: We apply the Cardano's formula to $(S)$ and we get the result.

Example: Set $(\beta, \xi, \alpha, \delta, \gamma, \rho, \sigma) \equiv(0.6,2.7,0.3,0.02,0.1,0.03,0.01)$.

This economy has.

$\mathrm{P}^{*}=P(\mathrm{r} *, \mathrm{~h} *, \mathrm{q} *) \simeq(0.03,0,0.9981,0.2250000000)$.

The computation of the eigenvalues of $\mathrm{J}\left(P^{*}\right)$ leads to $\lambda 1 \simeq 0.1970612707$, $\lambda_{2} \simeq-0.2753036128, \lambda_{3} \simeq 0.3412714849$ with $|\lambda 1|>\lambda_{3}$, and $\left|\lambda_{2}\right|>\lambda_{3}$.

\section{The existence of a homoclinic orbit}

The second step of our calculations is the explicit calculus of the homoclinic orbit in $\mathrm{J}\left(\mathbf{P}^{*}\right)$.

Theorem: Existence of homoclinic orbits to the real saddle in $\mathbf{P}^{*}$.Let $\omega \in \Omega_{\mathrm{IR}}$. Then

$$
\Omega 1_{\mathrm{H}}=\left\{\omega \in \Omega_{\mathrm{IR}}:(\mathrm{S}) \text { possesses a homoclinic orbit } \Gamma\left(\mathbf{P}^{*}\right)\right\} \neq \varnothing .
$$

In order to construct the homoclinic orbit analytically, we apply the procedure developed by [5]. We compute the stable and unstable manifolds, of the saddle equilibrium point $\mathrm{J}\left(\mathrm{P}^{*}\right)$, respectively, $W^{\mathrm{s}}$ and $W^{\mathrm{u}}$, with the undetermined coefficients method. We show that a homoclinic loop emerges as a solution trajectory of system (S) for parameter values belonging to the set $\Omega_{1} \mathrm{H} \subset \Omega_{\mathrm{IR}}$. The application of the method leads to the following relationship:

$$
\phi(\zeta)=\psi^{2}\left(\frac{F_{3 d}}{\left.\lambda_{3}+2 \lambda_{1}\right)}\right)+\left(\frac{1}{\lambda_{3}}\right) \psi^{2}\left(\frac{F_{2 d}\left(\lambda^{2}+2 \lambda^{3}\right)}{\left(F_{2 f}\left(\lambda^{2}-2 \lambda^{1}\right)\right)}\right) F_{2 f}=0
$$




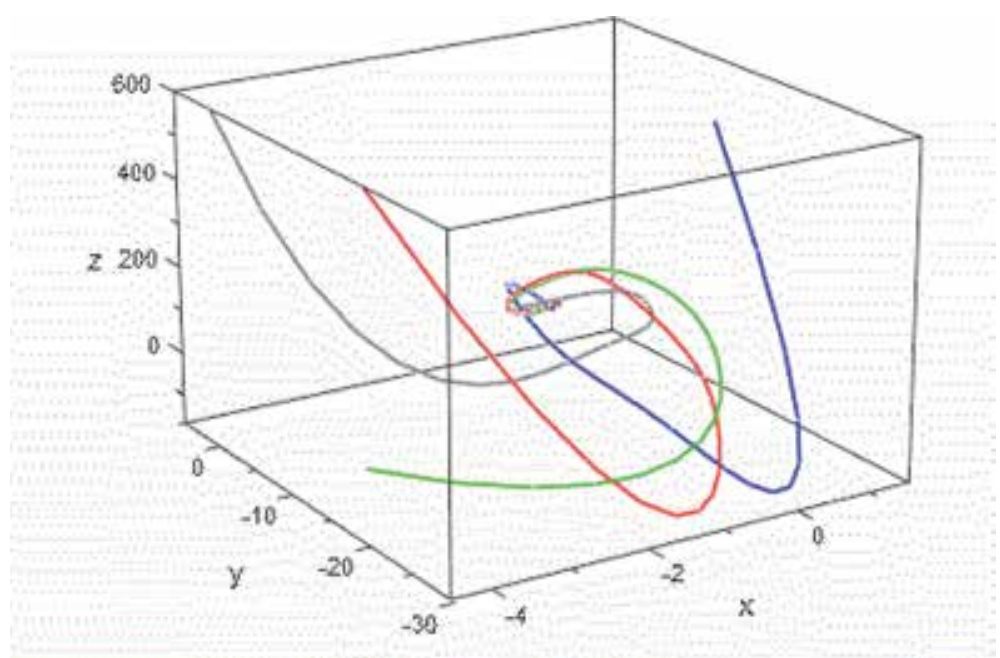

Figure 1.

A neighborhood of the homoclinic orbit.

where $\zeta$ and $\psi$ are arbitrary constants with $(\xi, \psi) \in(0,1)^{2}$, the $F \mathrm{i}, \mathrm{j}$ coefficients, $\mathrm{i}=1,2,3$ and $\mathrm{j}=\mathrm{e}, \mathrm{d}, \mathrm{f}$, and $F \mathrm{i}, \mathrm{j}$ are intricate combinations of the original parameters of the model and of three scaling factors $\left(C 1, C_{2}, C_{3}\right)$ associated with the choice of the eigenvectors. We now introduce a normal topological form for homoclinic bifurcation (see [7]). We consider a two-dimensional cross-section $\Sigma$ with coordinates $(\zeta, \psi)$ of $W^{\mathrm{u}}$, the unidimensional unstable manifold. Suppose that $\zeta=0$ corresponds to the intersection of $\Sigma$ with the stable manifold $W^{s}$ of $P^{*}$. Let conversely the point with coordinates $\left(\zeta^{\mathrm{u}} \psi^{\mathrm{u}}\right)$ correspond to the intersection of $W^{\mathrm{u}}$, with $\Sigma$. Then, the following occurs.

Definition: A split function can be defined as $\phi=\zeta^{\mathrm{u}}$. Its zero $\phi=0$ gives a condition for the homoclinic bifurcation in $\mathbb{R}^{3}$.

It might be impossible to characterize the system for a full set of parameter spaces and the boundary of the homoclinic orbit region. Using $\sigma$ as a bifurcation parameter, a homoclinic orbit can emerge as solution trajectories of the system (S). We observe a parameter set that remains inside $\Omega_{1 \mathrm{H}}$.

We found this result after many maple simulations (see Figure 1).

\section{Conclusions}

In this paper from an economic point of view, we show that a low inverse intertemporal elasticity of substitution plays a crucial role in determining global indeterminacy. We have focused on the parameter regions around a saddle equilibrium point with purely real eigenvalues.

We have applied the procedure developed by [5], and we have shown that a homoclinic loop emerges as a solution trajectory of the reduced system for an economic set of parameter values.

In order to get a homoclinic bifurcation, we have introduced a normal topological form. By varying the exponent of the inverse of elasticity of substitution, we consider a homoclinic bifurcation of dimension one.

As clearly pointed out in the literature [8], the homoclinic orbit connecting the unique steady state to itself implies the existence of a tubular neighborhood of the original homoclinic orbit. Any initial condition starting inside this tubular 
neighborhood gives rise to perfect-foresight equilibrium. Finally, with similar arguments introduced in [9], we are able to show global indeterminacy of the equilibrium for the model, since the result is valid beyond the small neighborhood relevant for the local analysis.

\section{Author details}

Beatrice Venturi

Department of Economics and Business, University of Cagliari, Cagliari, Italy

*Address all correspondence to: venturi@unica.it

\section{IntechOpen}

(C) 2019 The Author(s). Licensee IntechOpen. This chapter is distributed under the terms of the Creative Commons Attribution License (http://creativecommons.org/licenses/ by/3.0), which permits unrestricted use, distribution, and reproduction in any medium, provided the original work is properly cited. (cc) BY 


\section{References}

[1] Slobodyan S. Indeterminacy and stability in a modified Romer model. Journal of Macroeconomics. 2007;29: 169-177. DOI: 10.1016/j.jmacro.2005. 08.001

[2] Romer PM. Endogenous technological change. Journal of Political Economy. 1990;98:71-103

[3] Benhabib J, Perli R, Xie D. Monopolistic competition, indeterminacy and growth. Ricerche Economiche. 1994;48: 279-298. DOI: 10.1016/0035-5054(94) 90009-4

[4] Mulligan CB, Sala I, Martin X. Transitional dynamics in two-sector models of endogenous growth. The Quarterly Journal of Economics. 1993; 108(3):739-773

[5] Shang D, Maoan H. The existence of homoclinic orbits to saddle-focus.

Applied Mathematics and Computation. 2005;163:621-631. DOI: 10.1016/j. amc.2004.04.004

[6] Bella G, Mattana P, Venturi B. Shilnikov chaos in the Lucas model of endogenous growth. Journal of Economic Theory. 2017;172(C):451-477. DOI: 10.1016/j.jet.2017.09.010. ISSN: 0022-0531

[7] Kuznetsov YA. Elements of Applied Bifurcation Theory. 3rd ed. New York: Springer-Verlag; 2004. DOI: 10.1007/ 978-1-4757-3978-7. 632p

[8] Aguirre P, Krauskopf B, Osinga HM. Global invariant manifolds near homoclinic orbits to a real saddle: (Non) orientability and flip bifurcation. SIAM Journal on Applied Dynamical Systems. 2013;12:1803-1846. DOI: 10.1137/ 130912542

[9] Bella G, Mattana P, Venturi B.

Globally indeterminate growth paths in the Lucas model of endogenous growth. Macroeconomic Dynamics. 2019;2019: 1-12. DOI: 10.1017/S1365100519000373 


\section{Edited by Paul Bracken}

The subject of chaos has invaded practically every area of the natural sciences. Weather patterns are referred to as chaotic. There are chemical reactions and chaotic evolution of insect populations. Atomic and molecular physics have also seen the emergence of the study of chaos in these microscopic domains. This book examines the issue of chaos in nonlinear and dynamical systems, quantum mechanics, biology, and economics. 